\title{
A Measurement of the Cross Section Ratio of the $W$ and $Z$ Electronic Decays and the Total Width of the $W$ with the DZero Detector
}

\author{
DISSERTATION \\ submitted in partial satisfaction of the requirements for the degree of \\ DOCTOR OF PHILOSOPHY \\ in Physics \\ by \\ Jamal Nabil Tarazi \\ Dissertation Committee: \\ Professor Andrew Lankford, Chair \\ Professor Jonas Schultz \\ Professor Dennis Silverman
}


(c) Jamal Nabil Tarazi, 1997

All rights reserved. 
To

Christine

and
My Parents 


\section{Contents}

List of Figures $\ldots \ldots \ldots \ldots \ldots \ldots \ldots \ldots \ldots \ldots \ldots \ldots$ viii

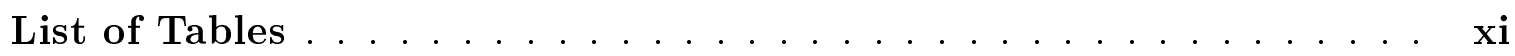

Acknowledgements $\ldots \ldots \ldots \ldots \ldots \ldots \ldots \ldots \ldots \ldots \ldots \ldots$

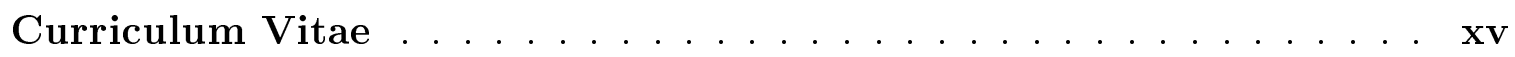

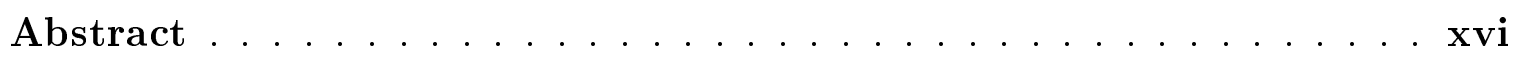

Chapter 1 Introduction and Theory $\ldots \ldots \ldots \ldots \ldots \ldots$

1.1 Overview of the Standard Model . . . . . . . . . . . . . . 1

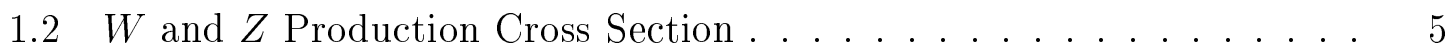

1.3 The $W$ Total Width . . . . . . . . . . . . . . . . . 9

1.4 Direct Measurement of ${ }_{w} \ldots \ldots \ldots \ldots \ldots \ldots \ldots$

1.5 Indirect Measurement of ${ }_{w} \ldots \ldots \ldots \ldots \ldots \ldots \ldots$

1.6 Previous Measurements of,$w \ldots \ldots \ldots \ldots \ldots \ldots$

1.7 Outline of the Dissertation . . . . . . . . . . . . 16

Chapter 2 Experimental Apparatus . . . . . . . . . . . . 17

2.1 Overview of the Tevatron Collider . . . . . . . . . . . 17

2.2 DØ Coordinate System . . . . . . . . . . . . . . . . . . 22

2.3 Overview of the D $\varnothing$ Detector $\ldots \ldots \ldots \ldots \ldots \ldots \ldots$

2.4 Central Tracking Detectors $\ldots \ldots \ldots \ldots \ldots \ldots \ldots$

2.4 .1 Vertex Drift Chamber . . . . . . . . . . . . . . . 30

2.4 .2 Transition Radiation Detector . . . . . . . . . . . . . . 32

2.4 .3 Central Drift Chamber . . . . . . . . . . . . . . . . 34

2.4.4 Forward Drift Chambers . . . . . . . . . . . . . . . 36

2.4.5 Central Detector Readout . . . . . . . . . . . . . . 38

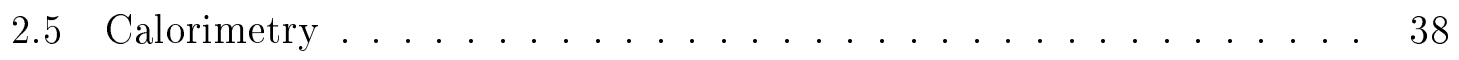

2.5.1 Calorimeter Design . . . . . . . . . . . . . . . . . . 43

2.5.2 Central Calorimeter . . . . . . . . . . . . . . . 47

2.5.3 Endcap Calorimeters . . . . . . . . . . . . . . . . 48

2.5.4 Intercryostat Detectors and Massless Gaps . . . . . . . . . 51

2.5.5 Calorimeter Readout . . . . . . . . . . . . . . . . . . . 52

2.5.6 Calorimeter Performance . . . . . . . . . . . . . 52

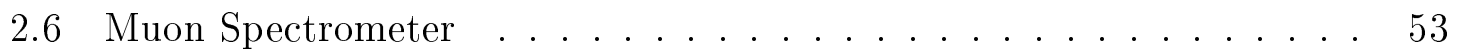

2.7 Trigger and Data Acquisition $\ldots \ldots \ldots \ldots \ldots \ldots \ldots$ 


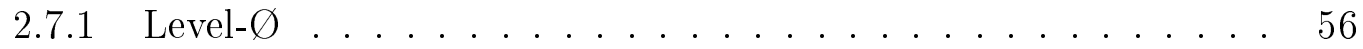

$2.7 .2 \quad$ Level-1 . . . . . . . . . . . . . . . . . . 58

2.7.2.1 Beam Vetoes . . . . . . . . . . . . . . 59

2.7.2.2 Level-1 Calorimeter Trigger . . . . . . . . . . . . . 60

2.7.2.3 Level-1.5 Calorimeter Trigger . . . . . . . . . . . . 62

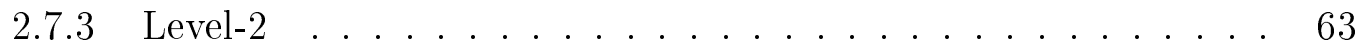

2.7.3.1 Level-2 Filter . . . . . . . . . . . . . . 65

2.8 Online Cluster . . . . . . . . . . . . . . . . . . . 66

2.9 Offline Data Processing . . . . . . . . . . . . . . 66

Chapter 3 Event Reconstruction and Particle Identification . . . . 69

3.1 Track Reconstruction . . . . . . . . . . . . . . . . . . . . 70

3.2 Vertex Finding . . . . . . . . . . . . . . . . . . 72

3.3 Calorimeter Hit Finding $\ldots \ldots \ldots \ldots \ldots \ldots$

3.4 Missing Energy . . . . . . . . . . . . . . . . . . . . 77

3.5 Jet Reconstruction . . . . . . . . . . . . . . . . . . . . . . 79

3.6 Electron Reconstruction . . . . . . . . . . . . . . . . . . . . 82

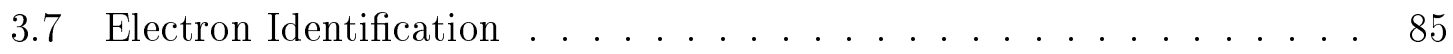

3.7.1 Electromagnetic Energy Fraction . . . . . . . . . . . . 86

3.7 .2 Shower Shape Analysis . . . . . . . . . . . . . . . . . . . 87

3.7 .3 Shower Isolation . . . . . . . . . . . . . . . . . . 90

3.7 .4 Track Matching. . . . . . . . . . . . . . . . . . . . . 92

3.8 Extension to Standard Electron ID . . . . . . . . . . . . . . 95

3.8.1 Electron Vertex Finding . . . . . . . . . . . . . . . . 95

3.8.2 Performance of Electron Vertex Finding . . . . . . . . . . 98

3.9 Neutrino Identification . . . . . . . . . . . . . . . . . . . . . . . . 99

Chapter 4 Event Samples . . . . . . . . . . . . . . . . . 103

4.1 Offline Electron Selection . . . . . . . . . . . . . . . 105

$4.2 \quad W \rightarrow e \nu$ Event Selection . . . . . . . . . . . . . . . . 107

4.2.1 $W \rightarrow e \nu$ Trigger Requirements . . . . . . . . . . . . 107

$4.2 .2 \quad W \rightarrow e \nu$ Offline Selection . . . . . . . . . . . . . . . 109

$4.3 Z \rightarrow e e$ Event Selection . . . . . . . . . . . . . . . . 111

4.3.1 $Z \rightarrow e e$ Trigger Requirements $\ldots \ldots \ldots \ldots \ldots \ldots \ldots$

4.3.2 $Z \rightarrow e e$ Offline Selection . . . . . . . . . . . . . . . 112

Chapter 5 Detector Acceptance . . . . . . . . . . . 115

5.1 Event Generation . . . . . . . . . . . . . . . . . . 116

$5.1 .1 \quad W$ and $Z$ Boson Generation . . . . . . . . . 116

5.1 .2 Transverse Momentum and Rapidity . . . . . . . . . . . 117

$5.1 .3 \quad W$ and $Z$ Decays . . . . . . . . . . . . . 120

5.1.3.1 QED Radiative Decays . . . . . . . . . . . 122

$5.1 .3 .2 \quad W \rightarrow \tau \nu \rightarrow e \nu \nu$ Decays . . . . . . . . . . . . . 122

5.2 Detector Response . . . . . . . . . . . . . . . . . . . . 123

5.2 .1 Electromagnetic Energy Scale . . . . . . . . . . . . . . 123

5.2.2 Electromagnetic Energy Resolutions . . . . . . . . . . . . . 124 
5.2.3 Hadronic Response and Resolutions _ . . . . . . . . . . 126

5.2 .3 .1 Hadronic Scale . . . . . . . . . . . . . 127

5.2 .3 .2 Hadronic resolution . . . . . . . . . . . . . 128

5.2 .4 Underlying Event Corrections . . . . . . . . . . . . . . 130

$5.3 \quad W$ and $Z$ Acceptances . . . . . . . . . . . . . . . . 132

5.3 .1 Systematic Errors . . . . . . . . . . . . . . 133

Chapter 6 Detection Efficiencies . . . . . . . . . . . . 137

6.1 Methods . . . . . . . . . . . . . . . . . . . 137

6.1.1 Diagnostic Data Sample . . . . . . . . . . . . . . . 139

6.1 .2 Background Determination . . . . . . . . . . . . 140

6.2 Single Electron Efficiencies . . . . . . . . . . . . . . . . . . . 142

6.3 Trigger Efficiency . . . . . . . . . . . . . . . . . . . . . 143

6.3 .1 Level- $\varnothing$ Efficiency . . . . . . . . . . . . . . . . . . . . 145

6.3.2 Level-2 $\mathbb{E}_{T}$ Trigger Efficiency . . . . . . . . . . . 146

6.4 Overall $W$ and $Z$ Efficiencies . . . . . . . . . . . . 147

$6.4 .1 W$ Selection Efficiency . . . . . . . . . . . . . . . 148

$6.4 .2 \quad Z$ Selection Efficiency . . . . . . . . . . . . . . . . . 149

6.4 .3 Efficiency Ratio. . . . . . . . . . . . . . . 150

Chapter 7 Backgrounds ................ . 153

7.1 Backgrounds in the $W \rightarrow e \nu$ Sample . . . . . . . . . . . . 153

7.1 .1 QCD Multi-jets . . . . . . . . . . . . . . . 154

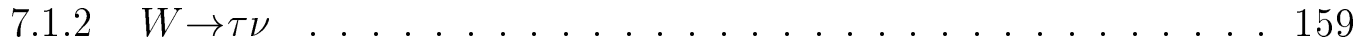

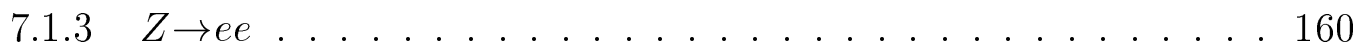

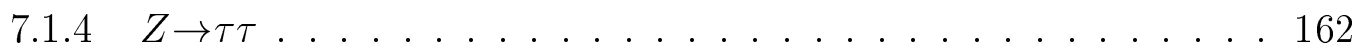

7.2 Backgrounds in the $Z \rightarrow e e$ Sample . . . . . . . . . . . 162

7.2 .1 QCD Backgrounds . . . . . . . . . . . . . 163

7.2.2 Drell-Yan and $Z / \gamma^{\star}$ Interference $\ldots \ldots \ldots \ldots$

$7.2 .3 \quad Z \rightarrow \tau \tau \ldots \ldots \ldots \ldots \ldots \ldots$. . . . . . . . . . . . . .

Chapter 8 Luminosity . . . . . . . . . . . . . . . 169

8.1 Determination of Instantaneous Luminosity . . . . . . . . . . . 169

8.1.1 Multiple Interaction Correction . . . . . . . . . . . . . 170

8.2 The Level- $\varnothing$ Monitor Constant $\sigma_{\mathrm{L} \varnothing} \ldots \ldots \ldots \ldots \ldots \ldots \ldots \ldots$

8.2.1 Inelastic Scattering Cross Sections . . . . . . . . . . . . 173

8.2.2 Level- $\varnothing$ Acceptances for Inelastic Scattering Processes . . . . . 175

8.2.3 The Level- $\varnothing$ Trigger Efficiency . . . . . . . . . . . . . 176

8.2.4 Correction for Multiple Single-Diffractive Interactions . . . . 177

8.2.5 Correction for Beam Halo . . . . . . . . . . . . . . . . 179

8.2.6 Calculation of $\sigma_{\mathrm{L} \varnothing} \ldots \ldots \ldots \ldots \ldots \ldots$

8.3 Determination of Integrated Luminosity . . . . . . . . . . . . 181

$8.3 .1 \quad$ Luminosity Integral . . . . . . . . . . . . . . . . . 182

8.4 Offline Luminosity Bookkeeping . . . . . . . . . . . . . . . . 185

8.5 Integrated Luminosity of the Data Samples . . . . . . . . . . . 186 
Chapter 9 Results and Conclusion . . . . . . . . . . . 189

9.1 Cross Section Calculation for $W$ Production . . . . . . . . . . . . 189

9.2 Cross Section Calculation for $Z$ Production . . . . . . . . . . 191

9.3 The Ratio $R$ of Cross Sections . . . . . . . . . . . . . 193

9.4 Extraction of the $W$ Width . . . . . . . . . . . . . . . . 194

9.5 Conclusions . . . . . . . . . . . . . . . . . . . . . 194

9.6 Future Prospects . . . . . . . . . . . . . . 196

Bibliography . . . . . . . . . . . . . . . . . 199 


\section{List of Figures}

$1.1 W$ and $Z$ gauge boson production processes. . . . . . . . . . 6

2.1 Fermilab Tevatron Collider complex. . . . . . . . . . . . . . 18

2.2 Isometric view of the $\mathrm{D} \emptyset$ detector. . . . . . . . . . . . . 25

2.3 Side view of the $\mathrm{D} \varnothing$ central tracking detectors. . . . . . . . . . . 28

2.4 Cross sectional view of the VTX chamber. . . . . . . . . . . 31

2.5 Cross sectional view of the first TRD layer. . . . . . . . . . . . . 33

2.6 Cross sectional view of the CDC . . . . . . . . . . . 35

2.7 Layout of the FDC. . . . . . . . . . . . . . . . 37

2.8 Isometric view of the $\mathrm{D} \emptyset$ calorimeter system. . . . . . . . . . . . . . . 44

2.9 Schematic view of a DØcalorimeter cell. . . . . . . . . . . . . . . . 45

2.10 Side view of the calorimeters . . . . . . . . . . . . . 46

2.11 Segmentation of the D $\varnothing$ calorimeter towers. . . . . . . . . . . . 46

2.12 D $\varnothing$ trigger and data acquisition system. . . . . . . . . . . 56

3.1 Vertex $z$ coordinate determination by the histogram method. . . . . . 73

3.2 Em fraction distribution for eletrons from $Z \rightarrow e e$ candidates and electrons in multi-jet triggered data. . . . . . . . . . . . . 87

$3.3 \chi_{h m}^{2}$ distribution for test beam electrons, test beam pions, and electrons from $W \rightarrow e \nu$ events. . . . . . . . . . . . . . . . . . 89

3.4 H-matrix $\chi_{h m}^{2}$ distribution for eletrons from $Z \rightarrow e e$ candidates and electrons in multi-jet triggered data. . . . . . . . . . . . . . . 90

3.5 Isolation distribution for eletrons from $Z \rightarrow e e$ candidates and electrons in multi-jet triggered data. . . . . . . . . . . . . . . . 91

3.6 Differences in cluster centroid and EM3 projected track positions for electrons from $Z \rightarrow e e$ candidates with $S_{t r k}<30$. . . . . . . 93

3.7 Definition of track match significance in terms of the cluster centroid in EM3 and the projection of the track to that radius . . . . . . . 93

3.8 Track match significance distribution for eletrons from $Z \rightarrow e e$ candidates and electrons in multi-jet triggered data. . . . . . . . . . . 94

3.9 Vertex determination by electron cluster-track projection method. . . 96

3.10 Distribution of $\left(z_{1}-z_{2}\right)$ in $Z \rightarrow e e$ events. . . . . . . . . . . . . 97

3.11 (a) Frequency at which the standard vertex is mismeasured as a function of instantaneous luminosity. (b) Invariant mass distribution for $Z \rightarrow e$ events using the two vertexing algorithms. . . . . . . . . . 99

4.1 The Run I integrated luminosity as a function of time. . . . . . . . . . 104

4.2 Transverse mass of the final $W \rightarrow e \nu$ candidate sample. . . . . . . . 110 
4.3 Invariant mass of the final $Z \rightarrow e e$ candidate sample. . . . . . . . . 113

5.1 Mass distribution from HERWIG showing the parton luminosity effect. 118

5.2 Lowest order (Drell-Yan) diagrams for $W$ and $Z$ production. . . . . . 119

5.3 Higher order diagrams for $W$ production: (left) the inital state gluon radiation process and the (right) Compton process. Similar diagrams exist for $Z$ production. . . . . . . . . . . . . . . . . . 120

5.4 Leading order diagrams for $W \rightarrow e \nu$ and $Z \rightarrow e e$ decays. . . . . . . . . . 121

5.5 (a) Simple Breit-Wigner convoluted with a gaussian resolution fit to the central $Z \rightarrow e e$ invariant mass spectrum. (b) Predicted $\sigma\left(M_{e e}\right)$ vs $\mathcal{C}$ for data and Monte Carlo for central electrons. . . . . . . . . . 125

5.6 Definition of the $\eta-\xi$ coordinate system in a $Z$ boson event. . . . . . . 128

5.7 (a) The fit to the $\eta$-balance in $Z \rightarrow e e$ events. (b) Predicted $\eta$-balance vs $\alpha_{H}$ values as obtained in CMS, and the $\eta$-balance for the data. . . .

5.8 (a) The $\eta$-balance width (RMS of the $\alpha_{H}$ corrected $\eta$-balance) in $Z \rightarrow e e$ events. (b) Predicted $\eta$-balance width vs $\alpha_{M B}$ for Monte Carlo and the data. . . . . . . . . . . . . . . . . .

6.1 Illustration of the background subtraction mechanism for the (a) sideband technique, and (b) fit technique. The dashed line shows the estimated level of background. . . . . . . . . . . . . . . . . 141

7.1 Diagram illustrating the two ways to split the parent sample in the determination of QCD multi-jet background to $W \rightarrow e \nu$ events. . . . . 155

$7.2 \quad \mathbb{E}_{T}$ spectra for the (a) signal and (b) background event samples in the single electron trigger. . . . . . . . . . . . . . . . 158

7.3 Electron $E_{T}$ distribution from $W \rightarrow \tau \nu \rightarrow e \nu \nu$ events. . . . . . . . . . 159

7.4 The $Z / \gamma$ mass distribution used in fitting the QCD background to $Z \rightarrow e e$ events. . . . . . . . . . . . . . 164

7.5 The QCD background distribution from "photon + jet" events, used in fitting the QCD background to $Z \rightarrow e e$ events. . . . . . . . . 165

7.6 Comparison of the $Z \rightarrow e e$ invariant mass distribution for the data, and the sum of Monte Carlo signal and predicted background. . . . . . . . 166

8.1 Schematic diagram illustrating the factorization principle used in calculating the double-diffractive cross section . . . . . . . . . . 174

8.2 Level- $\varnothing$ trigger efficiency as a function of instantaneous luminosity. . . 177

8.3 Distribution of the integrated luminosity of the entire data sample as a function of instantaneous luminosity. . . . . . . . . . 177

8.4 The average multiple single diffractive correction as a function of instantaneous luminosity. . . . . . . . . . . . . 178

8.5 The running average multiple single diffractive correction as a function of instantaneous luminosity. . . . . . . . . . . . . . 178

8.6 The beam halo correction as a function of instantaneous luminosity. . 179

8.7 The running beam halo correction as a function of instantaneous luminosity. . . . . . . . . . . . . . . . . . 179 
8.8 The combined beam halo and multiple single diffractive correction as a function of instantaneous luminosity. . . . . . . . . . . . 180

8.9 The running combined beam halo and multiple single diffractive correction as a function of instantaneous luminosity. . . . . . . . . 180 


\section{List of Tables}

1.1 Fundamental fermions in the Standard Model: leptons. . . . . . . . . . 2

1.2 Fundamental fermions in the Standard Model: quarks. . . . . . . . . . 3

1.3 Gauge bosons in the Standard Model. . . . . . . . . . . . . . 4

1.4 SM predictions of the total $W$ and $Z$ production cross sections at three different perturbation levels, with the MRSAp structure function. . . . 9

1.5 Known decay modes and relative decay rates of the $W^{+} \ldots \ldots$. . . 11

1.6 Previous measurements of , $W$, including the current world average. . . 15

2.1 Vertex Chamber parameters. . . . . . . . . . . . 31

2.2 Central Drift Chamber parameters. . . . . . . . . . . . . . . 35

2.3 Forward Drift Chamber parameters. . . . . . . . . . . . . 37

2.4 Central Calorimeter parameters. . . . . . . . . . . . 48

2.5 Endcap Calorimeter parameters. . . . . . . . . . . . 50

3.1 EM scale factors applied to each calorimeter cryostat. . . . . . . . 100

4.1 Summary of the $W \rightarrow e \nu$ signal event sample and topological breakdown.110

4.2 Summary of the $Z \rightarrow e e$ signal event sample and topological breakdown. 113

5.1 EM energy calibration constants applied to the data and in the CMS Monte Carlo. . . . . . . . . . . . . . . . . . . 124

5.2 EM energy resolution parameters used in the CMS Monte Carlo. . . . 125

5.3 Hadronic scale factors and resolutions used in the CMS Monte Carlo. . 130

5.4 Absolute and relative acceptances of $Z$ and $W$ boson events. . . . . 133

5.5 Systematic errors on the acceptance of $W$ and $Z$ boson events. . . . . 134

6.1 Summary of the background subtraction methods used in estimating electron selection efficiencies. . . . . . . . . . . . . . . . 142

6.2 Number of (background subtracted) central (CC) and forward (EC) electrons contained in the $Z \rightarrow e e$ signal window for various offline cuts and background subtraction methods. . . . . . . . . . . . . 144

6.3 Central electron relative efficiencies. . . . . . . . . . . . . . . . . . . 144

6.4 Forward electron relative efficiencies. . . . . . . . . . . . . . . 144

6.5 Overall $W$ electron selection efficiency. . . . . . . . . . . . . . . 149

6.6 Overall $Z$ electron selection efficiency. . . . . . . . . . . . . . 150

6.7 Ratio of $W$ and $Z$ selection efficiencies. . . . . . . . . . . . . 151

7.1 Measured parameters used as input to the QCD multi-jet fraction determination in $W \rightarrow e \nu$ events. . . . . . . . . . . . 157 
8.1 CDF and E710 $p \bar{p}$ total cross section components and their 'World Average'. . . . . . . . . . . . . . . . . . . . . 173

8.2 Double-diffractive cross section calculation. . . . . . . . . . . 175

8.3 Results of the Monte Carlo Level- $\varnothing$ acceptances. . . . . . . . . . . 176

8.4 Results of the calculation of the luminosity weighted average $\sigma_{\mathrm{L} \varnothing} \ldots 181$

8.5 Integrated luminosity results for the $W \rightarrow e \nu$ and $Z \rightarrow e e$ data samples. 187

9.1 Summary of input parameters to the $W \rightarrow e \nu$ cross section results. . . 190

9.2 Summary of input parameters to the $Z \rightarrow e e$ cross section results. . . 192

9.3 Summary of input parameters to the cross section ratio $R$. . . . . . 193 


\section{Acknowledgements}

Traditionally, this is the place where one reflects back on the long and winding path that leads to a Ph.D. Indeed, I find that what I have achieved would certainly not have been possible without the help of many many people:

I wish to express my gratitude to my advisor Andy Lankford for his support and guidance throughout my graduate career. Andy always gave me the freedom to pursue my interests, yet was always present when I needed any advice. I also wish to thank Anna Lipniacka for her patience while I embarked on my first HEP project (oh, those long forgotten SSC days...), and Dave Stoker for always asking tough questions and (thankfully) having some answers. I thank Jonas Schultz and Dennis Silverman, for teaching me the basics of particle physics and later serving on my doctoral committee.

I am grateful to all my D $\varnothing$ collaborators at Fermilab: they designed, constructed and operated one of the best experiments in the world. In addition, the members, leaders, and students of the Electroweak group were the brightest and most congenial. I thank them all for their help. This analysis, though, would have never finished if not for the help and guidance of Joey Thompson. Not only is he an excellent physicist and teacher, but the kindest and most patient person I have met. He truly embodies all the characteristics that define a Human Being. I will always remain endebted to him for leading me to the light at the end of the tunnel. Putting up with a "boob" like me must have been quite a challenge! I am also grateful to Paul Quintas for effortlessly spotting those $\mathcal{O}(0.1 \%)$ errors in my cross section tables, and helping out as much as he did.

A special thanks to Tom Ferbel for organizing the St. Croix summer school. Those two weeks will not only be remembered for the physics lectures, but for having met all those the new friends as well: it is quite ironic that one has to travel to the Virgin Islands in order to meet students from the other side of the Fermilab Tevatron... For anyone reading this page: do NOT miss out on that school!

I have been fortunate to meet many new friends, and I would like to express my gratitude to all of them. They are too numerous to list, but I would like to single out Ian Adam, Gene Alvarez, (Lord) Jim Cochran, the CINVESTAV connection, Eric Flattum, Gervasio Gomez, Greg Landsberg, Miguel Mostafa, Danilo Puseljic, and Joey Thompson. 
I thank the Prerna soccer team for giving me an outlet to safely release all the frustrations that build up during the week. Prerna - which means to inspire in Sanskrit - truly lived up to its name: a hodge podge of players with differing skill levels, who figured out early on how to cover up each other's individual weaknesses by playing as one unit on the field. Becoming league champions in only two years was inevitable! The names of the Prerna warriors Bao, Dante, Helios, Morpheus, Musashi, Tandava, Thor, Trimurthi, and Zeus will forever remain with me. They were my colleagues, my teammates, and most of all, my friends. The majority has moved on (to NY, NJ, CA, NM, Korea, or Japan), leaving a few of us at Fermilab to remember the glory days ... I would also like to thank the players on my current team, Fermions (the other D $\varnothing$ team), for accepting to absorb those remaining Prerna players.

Traveling back in time to the days at UC Irvine, I would like to thank Greg Griffin and Eric Van Drunen for sticking it out with me while preparing for the qualifiers! Despite having prepared so diligently, those six weeks of hell will mostly be remembered for the panic that struck us on the eve of the exam. Ted Brummel and Evangelos Papamichail (as well as the rest of the Quenya gang) represented a healthy - and at times quite unhealthy — escape from physics.

I would also like to thank my friends from my undergraduate years: Olivier, Mark, Carl, Ehsan, Manu, John, Jan \& Timbo (as well as Doc and Bo), Anu, ...

Perhaps my greatest gratitude goes to my family: my parents Leila and Nabil, my siblings Rana, Lama and Sari, all my uncles, aunts and cousins, as well as the extended family. Their love and support during my 22 years in school have been unyielding. I can only hope to give back to them as much as they have given me.

Last, and certainly not least, I would like to thank my wife Christine. She is the rock that I lean on and the beacon that guides me through my life. I will always be grateful to her for sticking it out these past five years and for always believing in me. You see babe, now we can start our real life! 


\section{Curriculum Vitae}

1991 B.A. in Physics, University of California, Berkeley

1991-1992 Teaching Assistant, Department of Physics and Astronomy, University of California, Irvine

1993-1997 Research Assistant, Department of Physics and Astronomy, University of California, Irvine

1994 M.S. in Physics, University of California, Irvine

1997 Ph.D. in Physics, University of California, Irvine Dissertation: A Measurement of the Cross Section Ratio of the $W$ and $Z$ Electronic Decays and the Total Width of the $W$ with the DZero Detector

Professor Andrew Lankford, Chair 


\title{
Abstract of the Dissertation
}

\section{A Measurement of the Cross Section Ratio of the $W$ and $Z$ Electronic Decays and the Total Width of the $W$ with the DZero Detector}

by

\author{
Jamal Nabil Tarazi \\ Doctor of Philosophy in Physics \\ University of California, Irvine, 1998 \\ Professor Andrew Lankford, Chair
}

This dissertation reports on the measurement of the inclusive production cross sections of electronically decaying $W$ and $Z$ gauge bosons in $p \bar{p}$ collisions at $\sqrt{\mathrm{s}}=1.8$ $\mathrm{TeV}$. The data was collected by the D $\varnothing$ detector during the 1994-1996 collider run of the Fermilab Tevatron. From a sample of $67406 \mathrm{~W} \rightarrow e \nu$, and $7140 Z \rightarrow e e$ candidates found in $82.4 \mathrm{pb}^{-1}$ and $108.5 \mathrm{pb}^{-1}$ of data respectively, the inclusive cross section times electronic branching fractions, and their ratio $R$ are measured to be:

$$
\begin{aligned}
\sigma_{W} \cdot \operatorname{Br}(W \rightarrow e \nu) & =2.322 \pm 0.009(\text { stat }) \pm 0.046(\text { syst }) \pm 0.123(\text { lum }) \mathrm{nb} \\
\sigma_{Z} \cdot \operatorname{Br}(Z \rightarrow e e) & =0.221 \pm 0.003(\text { stat }) \pm 0.003(\text { syst }) \pm 0.012(\text { lum }) \mathrm{nb} \\
R & =10.51 \pm 0.13(\text { stat }) \pm 0.14(\text { syst })
\end{aligned}
$$

Using this ratio $R$ and assuming Standard Model couplings, an indirect determination of the $W$ electronic branching fraction, $\operatorname{Br}(W \rightarrow e \nu)$, as well as the total $W$ decay width,,$W$, are obtained:

$$
\begin{gathered}
\operatorname{Br}(W \rightarrow e \nu)=(10.75 \pm 0.21) \% \\
, W=2.11 \pm 0.04 \mathrm{GeV}
\end{gathered}
$$

This enables an upper limit of $93 \mathrm{MeV}$ to be set on the contribution of unexpected decays to,$w$ at a confidence level of $95 \%$. 


\section{Chapter 1}

\section{Introduction and Theory}

This chapter briefly surveys the theoretical framework underlying this analysis, and then moves on to discuss the theoretical issues directly related to this analysis.

\subsection{Overview of the Standard Model}

The Standard Model (SM) of particle physics is a Quantum Field Theory based on the idea of local gauge invariance [1]. The gauge symmetry group of the SM is $S U(3)_{C} \otimes S U(2)_{L} \otimes U(1)_{Y}$, where $S U(3)_{C}$ is the symmetry group of the strong interactions, and $S U(2)_{L} \otimes U(1)_{Y}$ is the symmetry group describing the weak and electromagnetic interactions.

The Standard Model treats the interactions as a field, and interprets the excitations in the fields as particles. Each separate field corresponds to a different type, or flavor, of a particle. There are two general classes of particles in the theory: 1) the

fundamental fermions which have spin- $\frac{1}{2}$, and 2) the gauge bosons which have spin-1. 
The fermions obey the Pauli exclusion principle, thus making up what is usually considered to be "matter." They are further subdivided into particles called leptons and quarks.

There are six flavors of leptons: the electron $(e)$, the muon $(\mu)$, the tau $(\tau)$, and their corresponding neutrinos $\nu_{e}, \nu_{\mu}$, and $\nu_{\tau}$. The leptons are grouped into 3 generations $\left(e, \nu_{e}\right),\left(\mu, \nu_{\mu}\right)$ and $\left(\tau, \nu_{\tau}\right)$. Each generation has similar properties, except that the masses increase with each successive generation. The charged leptons interact via the electromagnetic and weak forces, while the uncharged neutrinos interact only by the weak force. Experimentally, the masses of the neutrinos are constrained to be quite small ${ }^{1}$, and the SM assumes that they are massless. Table 1.1 summarizes the main properties of the leptons.

Table 1.1: Fundamental fermions in the Standard Model: leptons.

\begin{tabular}{ccc}
\hline \hline Lepton & Mass $\left[\mathrm{GeV} / \mathrm{c}^{2}\right]$ & Charge $[\mathrm{e}]$ \\
\hline$e$ & 0.5110 & -1 \\
$\nu_{e}$ & $<15 \cdot 10^{-6}$ & 0 \\
\hline$\mu$ & 105.7 & -1 \\
$\nu_{\mu}$ & $<0.17$ & 0 \\
\hline$\tau$ & 1777 & -1 \\
$\nu_{\tau}$ & $<24$ & 0 \\
\hline \hline
\end{tabular}

There are also six flavors of quarks: up $(u)$, down $(d)$, charm $(c)$, strange $(s)$, top $(t)$, and bottom $(b)$. Unlike the leptons, they possess fractional electric charge either $-1 / 3 e$ or $2 / 3 e$, where $e$ is the charge of the electron. They are also distinct in that they possess an internal degree of freedom called color, which can take on three

\footnotetext{
${ }^{1}$ To date, no experiment has been able to detect a non-zero neutrino mass.
} 
possible values. They consequently feel the strong force, which binds quarks together and builds nucleons and mesons. They can also interact via the electromagnetic or weak force. The quarks are also grouped into three generations $(u, d),(c, s)$, and $(t, b)$, with each generation having similar properties, except the masses of the quarks which increase with each successive generation. Table 1.2 summarizes the main properties of the quarks.

Table 1.2: Fundamental fermions in the Standard Model: quarks.

\begin{tabular}{ccc}
\hline \hline Quark & Mass $\left[\mathrm{GeV} / \mathrm{c}^{2}\right]$ & Charge [e] \\
\hline$u$ & $2-8 \cdot 10^{-3}$ & $2 / 3$ \\
$d$ & $5-15 \cdot 10^{-3}$ & $-1 / 3$ \\
\hline$c$ & $1.0-1.6$ & $2 / 3$ \\
$s$ & $0.1-0.3$ & $-1 / 3$ \\
\hline$t$ & 180 & $2 / 3$ \\
$b$ & $4.1-4.5$ & $-1 / 3$ \\
\hline \hline
\end{tabular}

The gauge bosons are the mediators of the forces between the different particles in the theory. An interaction between two particles is viewed as a process in which these two particles exchange a virtual gauge boson. The main properties of the gauge bosons are summarized in Table 1.3.

Electromagnetism is mediated by the photon $(\gamma)$, and is described by Quantum Electrodynamics (QED). Any two charged particles interact by coupling to the photon. Since the photon is massless, the electromagnetic interaction has very long range. An interesting feature of electromagnetism is the "running" of the coupling strength: it increases as the energy involved in the interaction increases. This running feature is not unique to QED, and appears in other sectors of the theory. 
The weak interaction is mediated by the $W^{ \pm}$and $Z^{0}$ bosons. Since these gauge bosons are massive (with masses around $100 \mathrm{GeV} / \mathrm{c}^{2}$ ), the weak interaction has a short range of $\sim 10^{-18} \mathrm{~m}$. In the Standard Model, the treatment of the electromagnetic and weak forces has been unified in the Glashow-Weinberg-Salam (GSW) model [2], and these interactions are referred to as electroweak interactions.

The strong force is mediated by the gluons, and is described by Quantum Chromodynamics (QCD) [3]. There are a total of eight gluons, which couple to particles possessing the color charge (these particles are the quarks and the gluons themselves). There are three possible color charges, conventionally called red, blue and green. As is the case in QED, the value of the coupling runs. However, the direction of the effect is opposite: the strength of the coupling decreases as the energy in the interaction increases. This feature is quite attractive, since it allows quarks to behave as free particles (asymptotic freedom) at energies typical of modern highenergy experiments $(E>10 \mathrm{GeV})$, and it allows the use of perturbative techniques in theoretical calculations of processes in this regime. However, at lower energies, the coupling strength becomes large enough that perturbation theory breaks down, and renders any perturbative calculation nearly impossible.

Table 1.3: Gauge bosons in the Standard Model.

\begin{tabular}{ccc}
\hline \hline Gauge boson & Mass $\left[\mathrm{GeV} / \mathrm{c}^{2}\right]$ & Charge $[\mathrm{e}]$ \\
\hline gluons & 0 & 0 \\
$\gamma$ & 0 & 0 \\
$W^{ \pm}$ & 80.45 & \pm 1 \\
$Z^{0}$ & 90.91 & 0 \\
\hline \hline
\end{tabular}


The remaining ingredient of the Standard Model is the spin-0 particle called the Higgs boson. Its existence is required by the introduction of spontaneous symmetry breaking (Higgs mechanism) into the Electroweak sector of the theory. The latter is necessary in providing masses to the $W$ and $Z$ gauge bosons, since the gauge symmetry of the fundamental theory requires them to be massless. The quarks and leptons can also acquire masses through this mechanism. If the SM is correct, the Higgs should appear as a real particle. To date, the Higgs boson has not been observed.

\section{2 $W$ and $Z$ Production Cross Section}

The dominant production mechanism for electroweak gauge bosons ( $W$ and $Z$ ) in $p \bar{p}$ collisions is the weak Drell-Yan process [4], where a quark and an antiquark annihilate to form an on-shell $W$ or $Z$ boson:

$$
q \bar{q}^{\prime} \rightarrow W \quad \text { or } \quad q \bar{q} \rightarrow Z
$$

Due to the large momentum transfer of the process, the spectator model can be used, where the partons not directly involved in the production of the $W$ or $Z$ process are ignored. However, an inclusive cross section measurement does not make any requirements on the jets or transverse momentum of the generated gauge boson, and hence is effectively integrating over all orders of QCD processes. Such production processes are illustrated in Figure 1.1, which shows the lowest order diagrams (Drell-Yan), as well as the next highest order in $\alpha_{S}$, in which the produced boson is accompanied by a quark or gluon jet via Compton scattering or initial state radiation. 

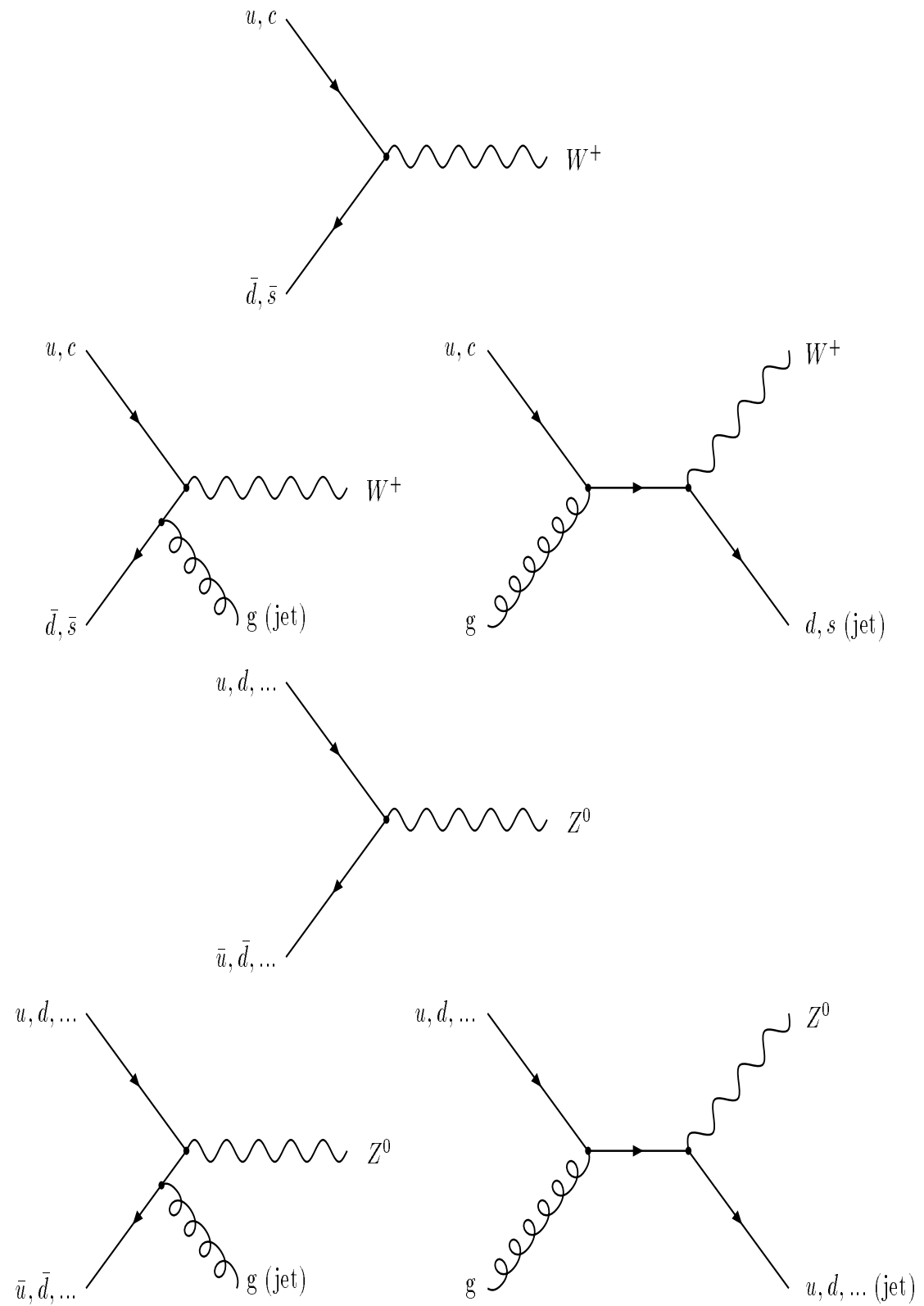

Figure 1.1: $W$ and $Z$ gauge boson production processes. 
The inclusive cross section for $W$ production can be computed from the sum of all production subprocesses. At Born level, the matrix element for the $W^{+}$production subprocess shown in Equation 1.1 is written down from the Feynman diagram rules [5, p. 247]:

$$
\mathcal{M}=-i V_{q q^{\prime}} \frac{g}{\sqrt{2}} \epsilon_{\mu} \bar{q}^{\prime} \gamma^{\mu} \frac{\left(1-\gamma_{5}\right)}{2} q
$$

where $g$ is the coupling strength ${ }^{2}, \epsilon_{\mu}$ is the polarization function of the $W, \bar{q}^{\prime}$ and $q$ represent the spinor wave functions for the quark and antiquark, and $V_{q q^{\prime}}$ is the Cabibbo-Kobayashi-Maskawa (CKM) matrix element connecting them. Summing $|\mathcal{M}|^{2}$ over $W$-polarizations, averaging over the initial quark spins, and integrating over the available phase space, leads to the $W^{+}$quark production subprocess cross section:

$$
\hat{\sigma}\left(q \bar{q}^{\prime} \rightarrow W^{+}\right)=2 \pi\left|V_{q q^{\prime}}\right|^{2} \frac{G_{F}}{\sqrt{2}} M_{W}^{2} \delta\left(\hat{s}-M_{W}^{2}\right)
$$

where $\hat{s}=\left(p_{q}+p_{\bar{q}^{\prime}}\right)^{2}$ is the square of the center of mass energy of the $q \bar{q}^{\prime}$ system, and $\delta$ is the Dirac delta function.

In order to obtain the inclusive $W$ production cross section, the subprocess cross section is convoluted with the proton structure function, including a color factor of $1 / 3$, and a factor of 2 to account for $W^{+}$and $W^{-}$production:

$$
\sigma(p \bar{p} \rightarrow W+X)=\frac{2 K_{W}\left(\alpha_{S}\right)}{3} \int_{0}^{1} d x_{a} \int_{0}^{1} d x_{b} \sum_{q} q\left(x_{a}, M_{W}^{2}\right) \bar{q}^{\prime}\left(x_{b}, M_{W}^{2}\right) \hat{\sigma}_{W^{+}}
$$

where $\hat{\sigma}_{W^{+}}=\hat{\sigma}\left(q \bar{q}^{\prime} \rightarrow W^{+}\right)$is derived in Equation 1.3. The factor $K_{W}\left(\alpha_{S}\right)$ incoporates QCD corrections which have been recently calculated to second order in $\alpha_{S}$ by

\footnotetext{
${ }^{2}$ The coupling strength $g$ is related to the $W$ mass, $M_{W}$, and the Fermi constant, $G_{F}$, via the relation: $g^{2}=8 M_{W}^{2} G_{F} / \sqrt{2}$.
} 
Hamberg, van Neerven, and Matsuura [6]. $x_{a}$ and $x_{b}$ are the momentum fractions of the quark $q$ in the proton $p$ and the quark $\bar{q}^{\prime}$ in the antiproton $\bar{p}$, respectively, while $q\left(x_{a}, M_{W}^{2}\right)$ and $\bar{q}^{\prime}\left(x_{b}, M_{W}^{2}\right)$ are the structure functions which describe the probability of finding the appropriate quark $q$ (or $\bar{q}^{\prime}$ ) with momentum fraction $x_{a}$ (or $x_{b}$ ) in the proton $p$ (or antiproton $\bar{p}$ ). A major source of uncertainty in the calculation of the cross section is due to the knowledge of these structure functions.

The inclusive $Z$ boson production cross section is derived in a similar fashion. The quark production subprocess cross section is [5, p. 251]:

$$
\hat{\sigma}(q \bar{q} \rightarrow Z)=\pi \frac{G_{F}}{\sqrt{2}}\left(1-4\left|Q_{q}\right| x_{W}+8 Q_{q}^{2} x_{W}^{2}\right) M_{Z}^{2} \delta\left(\hat{s}-M_{Z}^{2}\right)
$$

where $Q_{q}$ is the charge of the quark, and $x_{W} \equiv \sin ^{2} \theta_{W}=1-M_{W}^{2} / M_{Z}^{2}$. The inclusive $Z$ production cross section then becomes:

$$
\sigma(p \bar{p} \rightarrow Z+X)=\frac{K_{Z}\left(\alpha_{S}\right)}{3} \int_{0}^{1} d x_{a} \int_{0}^{1} d x_{b} \sum_{q} q\left(x_{a}, M_{Z}^{2}\right) \bar{q}\left(x_{b}, M_{Z}^{2}\right) \hat{\sigma}_{Z}
$$

where the variables are as defined for the $W$ case.

As mentioned previously, these inclusive production cross sections have been calulated to $\mathcal{O}\left(\alpha_{S}^{2}\right)$ in the form of $K$ factors [6]. Most higher order corrections to $W$ and $Z$ production are common to both processes, making the ratio of cross sections one of the most reliably calculated results of QCD. The only difference between higher order corrections to the $W$ and $Z$ cross sections stems from the fact that the $W$ boson couples exclusively to flavor-nonsinglet channels while the $Z$ boson couples to flavorsinglet channels as well ${ }^{3}$. This contribution vanishes, however, if the $u$ and $d$ quarks

\footnotetext{
${ }^{3}$ For example, two gluons can produce a $Z$ via a triangle quark loop with the $Z$ occupying the third vertex. This is not allowed for $W$ bosons.
} 
have the same mass. The ratio can then be expressed as [7]:

$$
\frac{\hat{\sigma}_{W}}{\hat{\sigma}_{Z}}=(\text { ratios of known couplings })+\mathcal{O}\left(\alpha_{S}^{2}\right)\left[\frac{m_{t}^{2}-m_{b}^{2}}{M_{Z}^{2}}\right]
$$

where the $\mathcal{O}\left(\alpha_{S}^{2}\right)$ terms have been calculated and are less than $1 \%$ of the ratio.

The program ZWPROD, provided by the authors of Reference [6], has been used to evaluate the theoretically predicted $W$ and $Z$ cross sections and their ratio. For the nominal value, the MRSAp structure function ${ }^{4}$ is used, with $M_{Z}=91.19 \mathrm{GeV} / \mathrm{c}^{2}$, $M_{W}=80.45 \mathrm{GeV} / \mathrm{c}^{2}$, and $\sin ^{2} \theta_{W}=0.2216$. The cross sections and their ratio at $\sqrt{\mathrm{s}}$ $=1.8 \mathrm{TeV}$ are listed in Table 1.4 .

Table 1.4: SM predictions of the total $W$ and $Z$ production cross sections at three different perturbation levels, with the MRSAp structure function.

\begin{tabular}{cccc}
\hline \hline Order & $\sigma_{W}[\mathrm{nb}]$ & $\sigma_{Z}[\mathrm{nb}]$ & $\sigma_{W} / \sigma_{Z}$ \\
\hline Born & 18.15 & 5.474 & 3.315 \\
$\mathcal{O}\left(\alpha_{S}\right)$ & 21.81 & 6.606 & 3.302 \\
$\mathcal{O}\left(\alpha_{S}^{2}\right)$ & 22.27 & 6.768 & 3.290 \\
\hline \hline
\end{tabular}

\subsection{The $W$ Total Width}

The decay widths of particles are one the simplest experimental observables, and in most cases, can be calculated theoretically with fairly high precision. The $W$ decay width is no exception: it can be expressed as a function of the basic electroweak parameters. In the electroweak sector of the Standard Model, three free parameters are

\footnotetext{
${ }^{4}$ All structure functions have been obtained from PDFLIB V7.07 [8]. MRSAp is identified by Ngroup $=3$, and Nset $=39$.
} 
required $^{5}$ to absorb the divergences occuring in radiative corrections. Consequently, these three parameters cannot be predicted by the theory, but once fixed, the values of other related parameters can now be computed to any desired order in perturbation theory. The most relevant parametrization for the kinematic regime of this analysis is the Sirlin on-shell renormalization scheme [9], which fixes the values of the $Z$ boson mass $\left(M_{Z}\right)$, the $W$ boson mass $\left(M_{W}\right)$, and the Fermi constant $\left(G_{F}\right)$.

At Born level, the amplitude for the electronic decay of the $W^{+}$boson is written down directly from Feynman diagram rules [5, p. 236]:

$$
\mathcal{M}=-i \frac{g}{\sqrt{2}} \epsilon_{\mu} \bar{e} \gamma^{\mu} \frac{\left(1-\gamma_{5}\right)}{2} \nu
$$

where $\epsilon_{\mu}$ is the polarization function of the $W$, and $\bar{e}, \nu$ represent the spinor wave functions for the positron and neutrino. Summing $|\mathcal{M}|^{2}$ over $W$-polarizations, averaging over the initial lepton spins, and integrating over the available phase space, leads to the partial decay width:

$$
,\left(W^{+} \rightarrow e^{+} \nu_{e}\right)=\frac{1}{48 \pi} g^{2} M_{W}=\frac{G_{F}}{\sqrt{2}} \frac{M_{W}^{3}}{6 \pi} \equiv, \stackrel{0}{W}
$$

where the electron is assumed to be massless, and $g^{2}=8 M_{W}^{2} G_{F} / \sqrt{2}$.

The total width of the $W$ boson can now be expressed in terms of,${ }_{W}^{0}$. A recent calculation which includes QCD corrections to $\mathcal{O}\left(\alpha_{S}\right)$ as well as electroweak radiative corrections finds [10]:

$$
{ }_{W}=\left[1+\delta\left(M_{t}, M_{H}\right)\right] \cdot\left[3+6\left(1+\frac{\alpha_{S}\left(M_{W}\right)}{\pi}\right)\right] \cdot{ }_{W}^{0} .
$$

\footnotetext{
${ }^{5}$ These are besides the fermion and Higgs masses and the CKM matrix elements.
} 
This is obtained by summing the partial widths of all known decay modes of the $W^{+}$, which are listed in Table 1.5 (the $W^{-}$decays to the charge conjugate pairs). All leptonic decay modes are equal to ${ }_{W}^{0}$. The decay modes to quark pairs involve the appropriate CKM matrix element, a color factor of 3, and the $\mathcal{O}\left(\alpha_{S}\right)$ QCD $K$-factor which accounts for gluon radiation [11]. The $\delta$ term represents the radiative corrections, and is dependent on the top quark mass $\left(M_{t}\right)$ and the Higgs mass $\left(M_{H}\right)$. These corrections are primarily due to vertex corrections; the propagator loop corrections are mostly absorbed by the $M_{W}$ term. The value of the $\delta$ term is about $-0.35 \%$ [10] when computed with $M_{t}=140 \mathrm{GeV} / \mathrm{c}^{2}$, and $M_{H}=100 \mathrm{GeV} / \mathrm{c}^{2}$.

Table 1.5: Known decay modes and relative decay rates of the $W^{+}$.

\begin{tabular}{cc}
\hline \hline $\begin{array}{c}\text { Decay mode } \\
\text { of } W^{+}\end{array}$ & $\begin{array}{c}\text { Partial width in units } \\
\text { of },{ }_{W}\end{array}$ \\
\hline$e^{+} \nu_{e}$ & 1.0 \\
$\mu^{+} \nu_{\mu}$ & 1.0 \\
$\tau^{+} \nu_{\tau}$ & 1.0 \\
$u \bar{d}$ & $3\left(1+\alpha_{S} / \pi\right) \cos ^{2} \theta_{C}$ \\
$u \bar{s}$ & $3\left(1+\alpha_{S} / \pi\right) \sin ^{2} \theta_{C}$ \\
$c \bar{s}$ & $3\left(1+\alpha_{S} / \pi\right) \cos ^{2} \theta_{C}$ \\
$c \bar{d}$ & $3\left(1+\alpha_{S} / \pi\right) \sin ^{2} \theta_{C}$ \\
\hline \hline
\end{tabular}

A measurement of the $W$ width is sensitive to the corrections described above and to new decay channels available to the $W$ that are not listed in Table 1.5. When the top quark mass is varied between $80 \mathrm{GeV} / \mathrm{c}^{2}$ and $200 \mathrm{GeV} / \mathrm{c}^{2}$, the $W$ width changes by 4\%; Higgs mass variations between $50 \mathrm{GeV} / \mathrm{c}^{2}$ and $1000 \mathrm{GeV} / \mathrm{c}^{2}$ cause a $1 \%$ change in ${ }_{W}[11]$. However, this varation of,$w$ on the top quark and Higgs masses is dependent on the renormalization scheme. Thus, in addition to testing the 
Standard Model at the next level of perturbation theory, a measurement of the $W$ width with a precision of $1 \%$ or better would begin to constrain the allowed values of these particles' masses through the radiative corrections.

The $W$ width could also be altered by new, unobserved decay modes of the $W$. By comparing the measured value of,$w$ to its theoretical prediction, one can set an upper limit on the excess width of the $W$ allowed by experiment for non-Standard Model decay processes, such as the decay of $W$ into supersymmetric charginos and neutralinos [12], or into heavy quarks [13].

\subsection{Direct Measurement of $\Gamma_{W}$}

In order to directly measure the width of the $W$ boson in $p \bar{p}$ collisions, one can pursue two avenues. A full reconstruction of a $W$ decay can be achieved in the hadronic channels, which account for $\sim 68 \%$ of the decays. However, the production of jets through ordinary QCD processes overwhelms the jets produced in $W$ decays by several orders of magnitude, rendering the experimental task of identifying a hadronically decaying $W$ virtually impossible.

In contrast, the signature of a high- $p_{T}$ lepton from the decay of the $W$ is quite distinctive in the environment of hadron collisions. However, the undetectable neutrino spoils the reconstruction of the invariant mass, since the longitudinal momentum of the neutrino cannot be measured. Therefore, the $W$ kinematic parameters are only reconstructed in the transverse plane, and a variable called the transverse mass, $m_{T}$, 
is introduced. It is defined by

$$
m_{T}^{2} \equiv\left(\left|\vec{p}_{T}^{e}\right|+\left|\vec{p}_{T}^{\nu}\right|\right)^{2}-\left(\vec{p}_{T}^{e}+\vec{p}_{T}^{\nu}\right)^{2}
$$

where $\vec{p}_{T}^{e}$ and $\vec{p}_{T}^{\nu}$ are the electron and neutrino momenta in the plane transverse to the $p$ and $\bar{p}$ beam directions. The transverse mass is analogous, in the transverse momentum subspace, to the invariant mass. It is invariant under longitudinal Lorentz transformations (boosts), and is only affected at second order by transverse boostS [14].

The transverse mass distribution for a given momentum transfer squared, $\hat{s}$, of a $W$ produced with zero $p_{T}$, is [5, pp. 259-263]

$$
\frac{d \hat{\sigma}}{d m_{T}^{2}}=\frac{\left|V_{q q^{\prime}}\right|^{2}}{4 \pi}\left(\frac{G_{F} M_{W}^{2}}{\sqrt{2}}\right)^{2} \frac{1}{\left(\hat{s}-M_{W}^{2}\right)^{2}+\left(,{ }_{W} M_{W}\right)^{2}} \frac{2-m_{T}^{2} / \hat{s}}{\sqrt{1-m_{T}^{2} / \hat{s}}} .
$$

The divergence, when $m_{T}^{2}=\hat{s}$, is known as the Jacobian edge and is characteristic of all two body decays. The majority of events, created with $m_{T}<M_{W}$, are in a region where the distribution is sensitive to the $W$ mass and the $W$ width. However, any measurement of this distribution is affected by the experimental energy resolutions which cause a large smearing of the Jacobian edge, effectively coupling the experimental resolution with the natural width of the $W$. In order to overcome this problem, any measurement of the width of the $W$ using the transverse mass distribution must be done with events populating the region $m_{T}>M_{W}$. In this region, the experimental resolutions are secondary to the natural width of the $W$ in determining the exact shape of the distribution. However, a very large sample of $W$ boson decays is necessary to achieve good precision, thus any measurement with the currently available $W$ data samples will be severely limited by the statistical error. 


\subsection{Indirect Measurement of $\Gamma_{W}$}

The $W$ width may be extracted from the measurement of the ratio, $R$, of the cross sections times branching fractions of electronically decaying $W$ and $Z$ bosons. This idea was first proposed by N. Cabibbo [15] in 1983 shortly after the discovery of the $W$ and $Z$ bosons by the UA1 [16] and UA2 [17] experiments. In theory, the ratio $R$ can be expressed as

$$
R \equiv \frac{\sigma \cdot \operatorname{Br}(p \bar{p} \rightarrow W \rightarrow e \nu)}{\sigma \cdot \operatorname{Br}(p \bar{p} \rightarrow Z \rightarrow e e)}=\frac{\sigma(p \bar{p} \rightarrow W)}{\sigma(p \bar{p} \rightarrow Z)} \cdot \frac{,(W \rightarrow e \nu)}{,(Z \rightarrow e e)} \cdot \frac{, Z}{{ }_{W}}
$$

On the right side of Equation 1.13, the ratio $\sigma_{W} / \sigma_{Z}$ of the production cross sections may be calculated from the boson couplings and knowledge of the proton stucture functions (see Section 1.2) with a small uncertainty. The $Z$ boson total and leptonic partial widths have been measured quite accurately by the LEP experiments [18]. Thus, a measurement of $R$ yields a precise measurement of the $W$ leptonic branching fraction, $(W \rightarrow e \nu) /,{ }_{W}$. By using a precise calculation of the $W$ partial width , $(W \rightarrow e \nu)$ (see Section 1.3), the value of the $W$ total width , $W$ can be extracted with very good precision.

In contrast to a direct measurement of the $W$ boson width, the ratio method is not sensitive to the electroweak corrections, since it is normalized to the calculated leptonic partial width, $(W \rightarrow e \nu)$. However, a more precise measurement can be achieved since the entire $W$ and $Z$ data samples are utilized. The advantage remains with the sensitivity of this mesurement to possible unknown decays of the $W$. 


\subsection{Previous Measurements of $\Gamma_{W}$}

To this date, the most precise measurements that have been made of the $W$ width have been extractions from the ratio $R$. Only one direct measurement has been performed [26], and its error is quite large. A summary of the measured values of ${ }_{W}$ is shown in Table 1.6.

Table 1.6: Previous measurements of , $w$, including the current world average, obtained from measurements indicated with a $(\star)$.

\begin{tabular}{ccccccc}
\hline \hline & Experiment & Year & Decay & $\sqrt{s}(\mathrm{GeV})$ & ${ }_{W}(\mathrm{GeV})$ & Method \\
\hline & $\mathrm{UA1}[19]$ & 1989 & $e$ & 546,630 & $2.8_{-2.0}^{+1.9}$ & Ratio \\
& $\mathrm{UA2}[20]$ & 1990 & $e$ & 546,630 & $2.30 \pm 0.20$ & Ratio \\
& $\mathrm{CDF}[21]$ & 1990 & $e$ & 1800 & $2.12 \pm 0.20$ & Ratio \\
$\star$ & $\mathrm{UA1}[23]$ & 1991 & $e$ & 546,630 & $2.18_{-0.24}^{+0.26}$ & Ratio \\
& $\mathrm{CDF}[22]$ & 1992 & $e, \mu$ & 1800 & $2.16 \pm 0.17$ & Ratio \\
$\star$ & $\mathrm{UA2}[24]$ & 1992 & $e$ & 546,630 & $2.10_{-0.16}^{+0.17}$ & Ratio \\
$\star$ & $\mathrm{CDF}[25]$ & 1995 & $e$ & 1800 & $2.11 \pm 0.32$ & Direct \\
$\star$ & $\mathrm{CDF}[26]$ & 1995 & $e$ & 1800 & $2.064 \pm 0.084$ & Ratio \\
$\star$ & $\mathrm{D} \varnothing[27]$ & 1995 & $e, \mu$ & 1800 & $2.044 \pm 0.093$ & Ratio \\
\hline \multicolumn{6}{c}{ World Average } & $\mathbf{2 . 0 7} \pm \mathbf{0 . 0 6}$ \\
\hline \hline
\end{tabular}

The best previous measurements of the $W$ width, from the CDF and D $\varnothing$ experiments, had errors of roughly $4.5 \%$. The current world average, which combines the latest published measurements of the CDF, DØ, UA1, and UA2 experiments, is

$$
{ }_{W}=2.07 \pm 0.06 \mathrm{GeV}
$$

and has a precision of $\frac{\delta \Gamma_{W}}{\Gamma_{W}}=2.9 \%$. The subject of this dissertation is the indirect measurement of the $W$ boson width with a precision that is better than the current world average. 


\subsection{Outline of the Dissertation}

The dissertation will proceed as follows: Chapter 2 discusses the experimental setup for this analysis, with emphasis placed on detector subsystems that affect the detection of electrons. Chapter 3 describes how the various final state particles are reconstructred and identified in the data sample collected by the detector. The next four chapters discuss the details of the analysis: Chapter 4 describes the selection of the candidate $W \rightarrow e \nu$ and $Z \rightarrow e e$ samples, Chapter 5 the detector's kinematic and fiducial acceptance, Chapter 6 the trigger and selection efficiencies, and Chapter 7 the amount of background present in the final candidate samples. Chapter 8 presents the details of the determination of the integrated luminosity. Finally, Chapter 9 will present the $W$ and $Z$ cross section results, their ratio, and the extraction of the total width of the $W$. 


\section{Chapter 2}

\section{Experimental Apparatus}

This chapter will briefly discuss the apparatus used: from the Tevatron beam to the actual DØ detector. Emphasis will be placed on detector systems that affect this analysis, namely the calorimeter and the central tracker. The interested reader is urged to consult the references given here for further details. The reference for the detector as a whole is [28], which provides a complete description.

\subsection{Overview of the Tevatron Collider}

The Fermilab Tevatron [29], located at Fermi National Accelerator Laboratory (FNAL), is currently the highest energy particle accelerator in the world with a center of mass energy of the colliding proton-antiproton system of $1.8 \mathrm{TeV}$. In fact, the Tevatron, shown in Figure 2.1, is only the last of a chain of accelerators in the FNAL collider complex that start with protons taken from hydrogen gas atoms and end with a beam whose energy is $900 \mathrm{GeV}$. The basic idea behind a particle accelerator is quite simple: a charged particle is given a boost when it crosses a gap immersed in 
an electromagnetic field. Linear accelerators consist of a series of such gaps arranged in a linear configuration. Alternatively, a single gap can be reused by containing the particles in a circular (or elliptic) orbit. Magnets are used to bend the particles' trajectories and keep them in the desired orbit. On each pass around the ring, the energy of the particles is increased; as the particles' momenta increase, the magnetic field is increased in a synchronized fashion thus enabling the particles to remain in the ring. This type of machine is called a synchrotron, of which the Tevatron is an example.

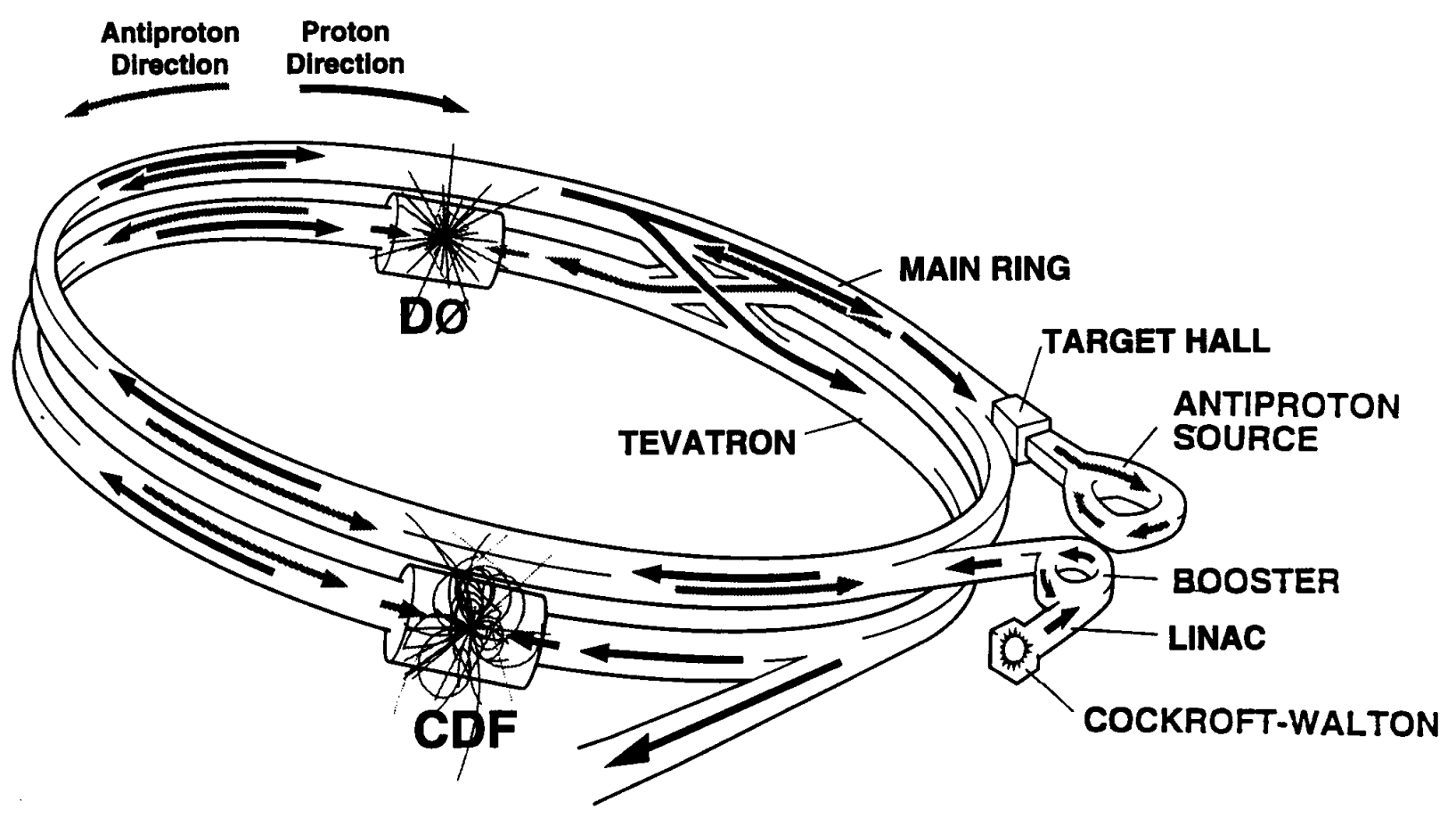

Figure 2.1: Fermilab Tevatron Collider complex.

After a beam of particles has been accelerated to the desired energy, it can be used in one of two ways: 1) fixed target mode or 2) collider mode. Each mode has its advantages and disadvantages, hence each is used for a different purpose. 
In the fixed target mode, the primary beam can be directed into detectors or onto various targets to produce a wide range of secondary beams (consisting of charged or neutral particles). The secondary beams are then delivered to detectors. The major advantage of the the fixed target mode is the control available to the researcher to change the particle type and energy. In the collider mode, the circulating beams of particles are strongly focussed so they may collide at specific interaction points. Detectors are built to surround these interaction points and collect the resultant debris of the collision. The advantage of the collider mode is the much higher center of mass energy that is made available ${ }^{1}$.

The Tevatron complex is actually a synchrotron of great complexity and sophistication (a more detailed introduction is provided in Reference [30]). It is composed of many parts:

1. The Preaccelerator

2. The Linac

3. The Booster

4. The Main Ring

5. The Antiproton Source

6. The Tevatron

\footnotetext{
${ }^{1}$ In fixed target mode, the maximum available center of mass energy for a given beam of energy $E$ is proportional to $\sqrt{E}$; in collider mode, it is proportional to $E$.
} 
The origin of the beam is a bottle of pressurized hydrogen gas. The hydrogen atoms are ionized by the addition of electrons, thus forming $H^{-}$ions. These $H^{-}$ions are accelerated to an energy of $750 \mathrm{keV}$ by an electrostatic Cockroft-Walton accelerator. Once at $750 \mathrm{keV}$, the ions are injected into the Linac. The Linac is a $150 \mathrm{~m}$ linear accelerator, which raises the energy of the ions to $400 \mathrm{MeV}$. Once the ions emerge from the Linac, they are passed through a carbon foil which strips off the electrons, thus creating protons. The protons are then steered into the Booster, a $151 \mathrm{~m}$ diameter synchrotron. Operating at a frequency of $15 \mathrm{~Hz}$, the Booster increases the energy of the protons to $8 \mathrm{GeV}$. The protons are then injected into the Main Ring, a $1 \mathrm{~km}$ radius synchrotron composed of 1000 conventional copper-coiled magnets. As the protons in the Main Ring are accelerated to $120 \mathrm{GeV}$, they are also being compressed into short bunches (with $\sim 2 \cdot 10^{12}$ protons/bunch). Some of the bunches are accelerated further to an energy of $150 \mathrm{GeV}$ for subsequent injection into the Tevatron. The remaining bunches, still at an energy of $120 \mathrm{GeV}$, are directed towards the Antiproton Source: the antiproton production cycle repeats every 2.4 seconds.

The production of antiprotrons (called pbar stacking) begins when $120 \mathrm{GeV}$ proton bunches are dumped on a nickel/copper target (in the Target Hall) and antiprotons are collected from the resultant debris. These antiprotons vary greatly in their angular divergence and energy spectrum. First, the beam is focussed using a lithium lens. A magnetic field is then applied to select $8 \mathrm{GeV}$ antiprotons that are transported into the Debuncher, the first of two antiproton storage rings. The production rate is about twenty antiprotons for every million protons that are sent 
to the Target Hall. The Debuncher uses sophisticated radio-frequency and cooling techniques to equalize all antiproton energies. This process is achieved by debunching then cooling the beam. Debunching is the process of rotating in phase space from a configuration with a small time and large momentum spread to one of large time and small momentum spread. Stochastic cooling [31] further reduces any momentum spread by applying a correction signal (via kicker electrodes) to the particles in the beam once any deviation of their trajectory from the desired orbit is measured. This process runs continuously until the next bunch of antiprotons arrives, about $2.4 \mathrm{~s}$ later. At this point, the monochromatic antiproton beam with $\sim 2 \cdot 10^{6}$ antiprotons is transfered to the second antiproton storage ring, known as the Accumulator. In the Accumulator, further cooling is applied to the antiprotons, and their density is increased. When about $4 \cdot 10^{11}$ antiprotons are stored (which typically takes 8 to 12 hours), they are transfered into the Main Ring where their energy is increased to $150 \mathrm{GeV}$, and are injected into the Tevatron in the direction opposite to that of the proton beam.

The Tevatron is located in the same tunnel as the Main Ring. They are separated by a distance of $1 \mathrm{~m}$, except at two intersection regions where detectors are located: B0 intersection region (separation is 19 feet) for the CDF detector and the D0 intersection region (separation is 89.2 inches) for the DØ detector. The Tevatron is a synchrotron composed of super-conducting magnets which operate at a temperature of $4.6 \mathrm{~K}$ and can produce fields of approximately 3 Tesla, thus allowing higher 
energy protons and antiprotons. In the final acceleration phase, six bunches of protons (with $\sim 10^{11}$ protons/bunch) and six bunches of antiprotons (with $\sim 5 \cdot 10^{10}$ antiprotons/bunch) are ramped to the maximum energy of $900 \mathrm{GeV}$. Once at this energy (called flattop), the beams are strongly focussed and made to collide at the B0 and D0 experimental areas. The proton and antiproton beams are kept from colliding at other points by the use of electrostatic separators. Over time, interactions of the beam with residual beam pipe gases cause a decrease in the size and density of the proton and antiproton bunches. The beam lifetime (also called store length) was typically 12 to 18 hours. Production of antiprotons is continuous during collisions in order to refill the Tevatron with new bunches as quickly as possible: typical down-time between any two stores was on the order of 2 hours.

\subsection{DØ Coordinate System}

Before proceeding with a description of the DØ detector, it is necessary to define the coordinate system used in the experiment. DØ uses a right-handed coordinate system with the positive $z$-axis pointing in the direction of the proton beam, and the positive $y$-axis pointing towards the zenith. The angular coordinates (azimuthal $\phi$ and polar $\theta$ ) are defined such as $\phi=0$ coincides with the $+x$ direction and $\theta=0$ with $+z$ direction. Radial distances are measured perpendicularly to the beam line. Instead of the angle $\theta$, it is convenient to use the pseudorapidity, $\eta$, defined by

$$
\eta \equiv-\ln \left[\tan \left(\frac{\theta}{2}\right)\right]=\tanh ^{-1}(\cos \theta)
$$


The pseudorapidity approximates the true rapidity ${ }^{2}$ of a particle,

$$
y=\frac{1}{2} \ln \left(\frac{E+p_{z}}{E-p_{z}}\right)
$$

in the limit that $m \ll E$, where $m$ is the particle's rest mass. In addition, it is often convenient to express polar angles in terms of "detector pseudorapidity", $\eta_{\text {det }}$, which is computed with respect to an interaction point whose longitudinal position is set to $z=0$. In reality, this interaction point is characterized by a Gaussian distribution whose mean is $z=0 \mathrm{~cm}$ and deviation $\sigma_{z}=30 \mathrm{~cm}$, thus causing a slight difference between $\eta$ and $\eta_{\text {det }}$ for any given particle.

In practice, it is also convenient to project the momentum vector of a particle onto a plane perpendicular to the beam line. This "transverse momentum"

$$
p_{T}=p \sin \theta
$$

is useful due to the fact that in a $\bar{p} p$ collision, many products of the collision escape detection by going down the beam pipe, thus making it impossible the measure the momenta accurately along the direction of the colliding partons. However, one can apply momentum conservation in the transverse plane. Similarly, one defines the "transverse energy" as

$$
E_{T}=E \sin \theta
$$

\footnotetext{
${ }^{2}$ The center of mass of the partons involved in the hard scatter is not necessarily at rest in the laboratory frame. In this case, for a parton of energy $E$ and momentum $p$, the rapidity as defined in Equation 2.2 is a Lorentz invariant quantity.
} 


\subsection{Overview of the $\mathrm{D} \varnothing$ Detector}

The DØ detector [28] is a large, general purpose detector for the study of high energy $\bar{p} p$ collisions at the Fermilab Tevatron collider. The detector was first proposed [32] in 1983, and has been in operation since 1992. The design was optimized for the study of high $p_{T}$ physics and high mass states. These topics include the search for the top quark, precision electroweak tests (production of $W$ and $Z$ gauge bosons), studies of perturbative QCD (production of jets, prompt photons and $b$-quark hadrons), and searches for new particles and phenomena beyond the Standard Model. With this choice of physics topics, the design stresses excellent identification of electron$\mathrm{s}$ and muons, a good measurement of high- $p_{T}$ jets, as well as the determination of any missing transverse energy. There is little attention paid to the identification or tracking of individual particles within jets.

Modern particle detectors at high energy hadron colliders (e.g., consult [33]) consist generally of three main detector elements: the tracking chamber, the calorimeter and the muon system. Closest to the interaction region is the tracking detector which is responsible for measuring the three dimensional trajectories of charged particles passing through it. Most often, it is immersed in a magnetic field thus permitting the determination of the momenta of the tracks (via a measurement of their bending radius). Surrounding the tracking detector is the calorimeter. This device enables the measurement of the energy of most particles that hit it. A calorimeter is made "thick" in order to absorb all of the energy of the incident particle; in contrast, the 
tracking detector contains the least possible material in order to minimize energy loss and multiple scattering prior to the calorimeter. Only the most penetrating of particles make it through a calorimeter: muons and neutrinos. Muons are detected by the presence of additional tracking chambers placed outside the calorimeter: any charged tracks originating from the interaction point and penetrating the calorimeter are most likely muons. Neutrinos, being non-interacting, are not detected in the tracking chambers nor is their energy deposited in the calorimeter. Their presence is inferred from an imbalance in the total detected transverse energy in the calorimeter. The calorimeter covers a large range in pseudorapidity thus making the detector as hermetic as possible. The only way energy can escape undetected is the very forward region, within the beam pipe traversing the center of the detector.

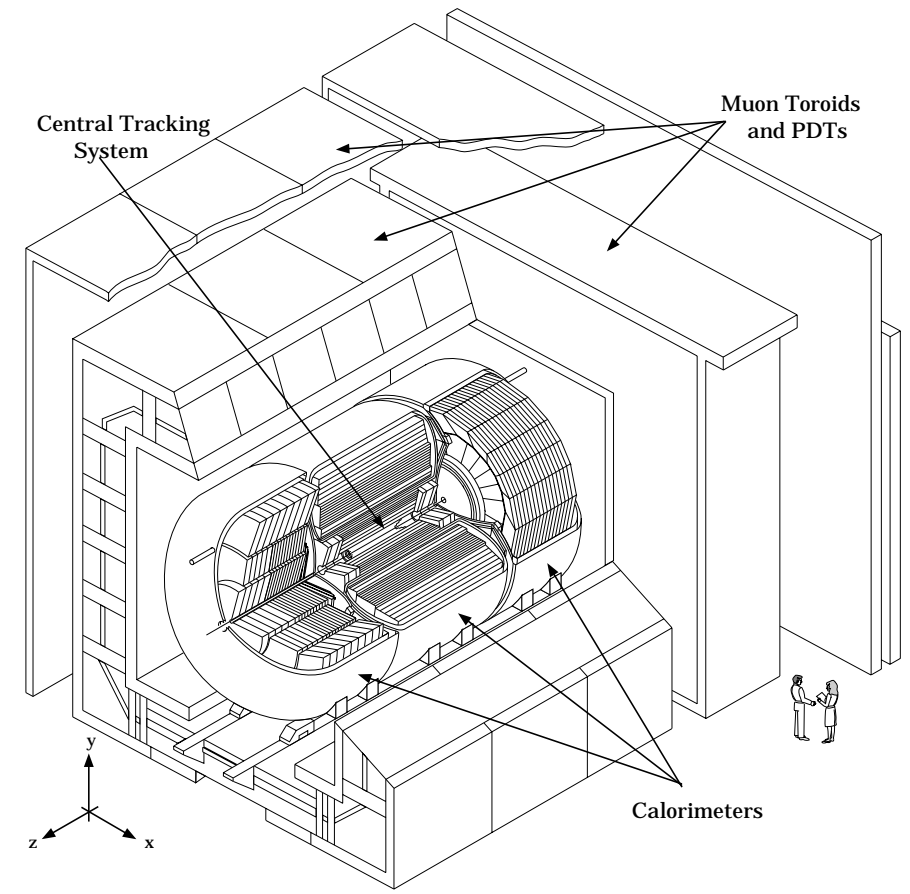

Figure 2.2: Isometric view of the D $\varnothing$ detector. 
The DØ detector, shown in Figure 2.2, is a modern collider detector which follows the prescription described above. Heavy emphasis is placed on the calorimetry. Hence, the tracking detectors, which surround the beam pipe, have no central magnetic field. Part of the reason for this is the fact that for high- $p_{T}$ objects, a better momentum resolution is achieved with a calorimeter than a tracking device ${ }^{3}$. The presence of a magnetic field also degrades the calorimetric energy measurement by: 1) sweeping away low momentum charged particles out of jets and 2) adding additional material (the solenoid magnet) prior to the calorimeter. Superior calorimetry is achieved at $\mathrm{D} \varnothing$ by using a liquid argon sampling calorimeter made mostly from depleted uranium. Since the tracking volume is smaller than usual (due to the absence of a magnet), the calorimeter is compact and yet remains very "thick". It covers pseudorapidity ranges out to $|\eta|<4.5$, making it almost hermetic. Its azimuthal symmetry is broken by the presence of the Main Ring beam pipe which passes through the upper portion of the calorimeter at a radius of 89.2 inches from Tevatron beam pipe (see Figure 2.2). To identify muons, additional tracking chambers were installed outside the calorimeter. A magnetized iron toroid was placed between the first two of three muon tracking layers, providing a measurement of the muon momentum. The geometry of these muon tracking layers is rectangular (thus violating azimuthal symmetry), and one layer is absent underneath the calorimeter, since space was needed for the detector's mechanical support structures.

\footnotetext{
${ }^{3}$ For a magnetic tracker, the momentum resolution $\frac{\delta p_{T}}{p_{T}} \propto p_{T}$; for a calorimeter, the energy resolution $\frac{\delta E}{E} \propto \frac{1}{E}$.
} 
The detector is very large. It weighs 5500 tons and measures $13 \mathrm{~m}$ (height) $\times$ $11 \mathrm{~m}$ (width) $\times 17 \mathrm{~m}$ (length). It is supported by a platform mounted on rollers so that the entire detector may be rolled from the assembly area to the Collision Hall. In addition, this platform provides space for detector electronics, as well other

utilities (such as gas, power, cryogenics). The use of digitizing electronics is kept to a minimum on the detector platform in order to reduce electronic noise. The analog signals are read via cables from this platform to the Moving Counting House (MCH). The MCH houses all the digitization electronics, the hardware trigger, high-voltage supplies, etc. In order to keep the cable length short, the MCH is likewise mounted on rollers, and follows the detector as it moves in and out of the Collision Hall. The cables are then led to the assembly building (Fixed Counting House) where the software trigger, online systems, and the control room are found.

The sections to follow will discuss the details of the different subsystems of the DØ detector.

\subsection{Central Tracking Detectors}

The Central Tracking Detectors (CD) at DØ are responsible for the reconstruction of the three-dimensional trajectories of charged particles which pass through them. The major design motivation is driven by the absence of a magnetic field. Without the need to measure the momenta of charged particles, emphasis is placed on good two-track resolving power, high efficiency, and good ionization measurement 
to distinguish between electrons and closely spaced conversion pairs $\left(\gamma \rightarrow e^{+} e^{-}\right)$. In addition, the central trackers are responsible for making a precise measurement of the location of the interaction vertices for each event, as well as improving the accuracy of the muon momentum measurements.

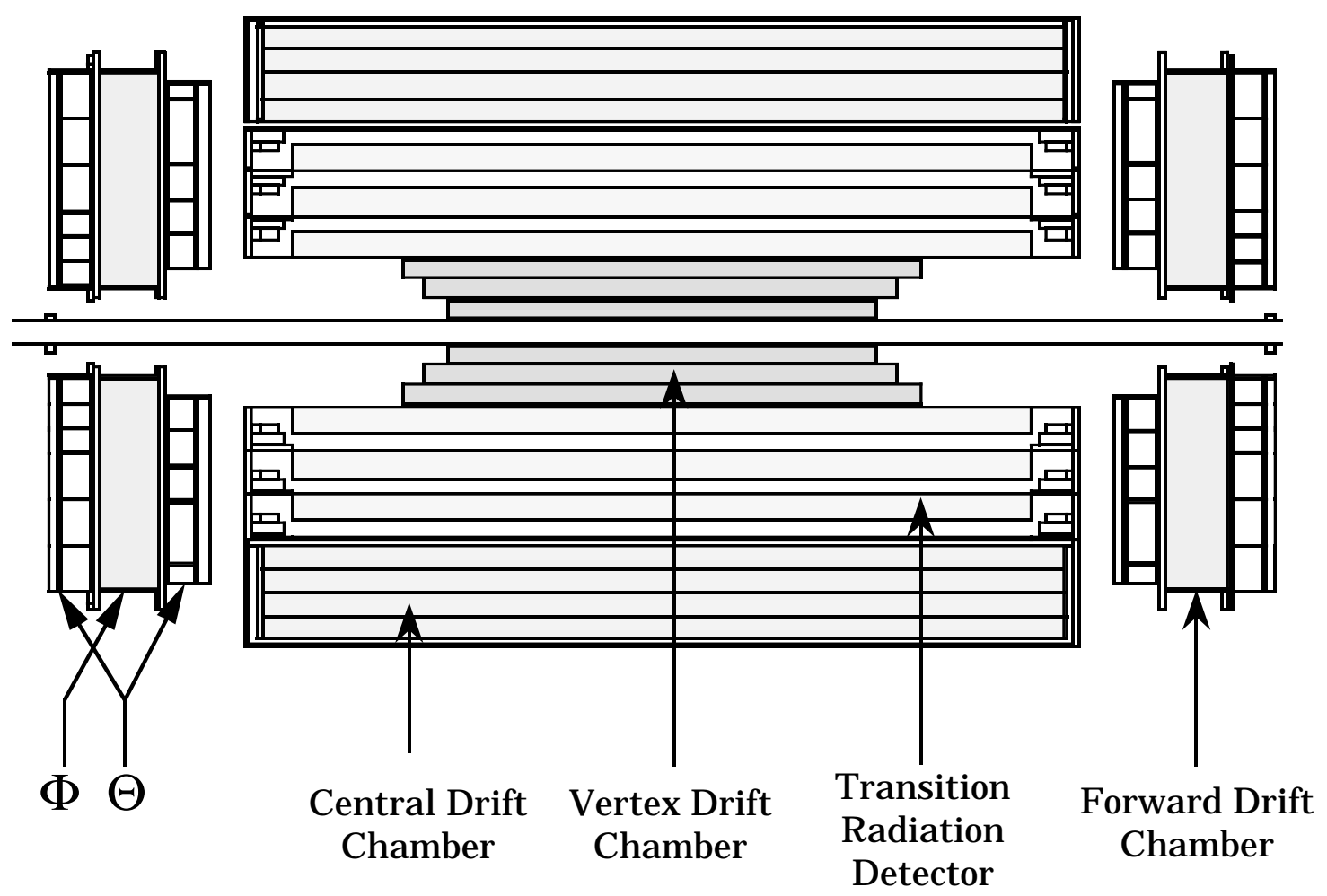

Figure 2.3: Side view of the DØ central tracking detectors.

The CD, shown in Figure 2.3, is composed of four subsystems. A Vertex Drift Chamber (VTX), a Transition Radiation Detector (TRD) and a Central Drift Chamber (CDC) are cylindrical devices which cover the large angle region, and are arranged concentrically around the beam pipe. The fourth subsystem consists of two Forward Drift Chambers (FDC) which are oriented perpendicularly to the beam pipe. The entire system is contained within the inner cylindrical aperture of the calorimeters and occupies a volume bounded by a radius $r=75 \mathrm{~cm}$ and length $l=270 \mathrm{~cm}$. 
Three of the four tracking detectors are wire drift chambers: the VTX, the CDC, and the FDC. The basic principle governing the operation of such devices will be outlined in the rest of this section. The reader is referred to [34] for detailed discussions on the principles of drift chambers.

When a charged particle passes through a gas, it interacts electromagnetically with nearby atomic electrons. This interaction causes the liberation of electron/ion pairs along the trajectory of the particle. In the presence of an electric field, the electrons will drift to the positively charged electrode wire, commonly known as a sense wire. The ions will drift in the opposite direction, but their motion can be ignored: their larger mass causes their drift velocity to be considerably slower than that of the electrons. A multi-wire drift chamber has several such sense wires strung in parallel. The ionization electrons will drift to the sense wire closest to their point of creation. The small diameter of the sense wire produces a very strong electric field in its immediate vicinity. Consequently, this strong electric field accelerates the drift electrons to energies high enough to induce further ionization. In this manner, the number of electrons increases exponentially and forms an avalanche, thus giving rise to a large and measurable electrical current whose size is proportional to the original number of electron/ion pairs created. The ratio between the final number of electrons collected and the initial number produced is referred to as the gas gain, and is typically on the order of $10^{4}-10^{6}$.

The velocity of the drift electrons is independent of the particle that initiates the ionization. It is dependent, however, on the strength of the electric field as well 
as the composition, temperature and pressure of the gas mixture. The drift time, defined as the difference between the known collision time and the arrival time of the pulse at the sense wire, is combined with the drift velocity in order to infer the drift distance of the electrons. In order to obtain a linear relationship between distance and time, it is necessary to ensure a constant electric field over as large a volume of the chamber as possible. Typically, one inserts additional field-shaping cathodes in order to make the field more uniform. From the drift time and inferred drift distance, the trajectories of charged particles are reconstructed (see Section 3.1).

For further discussion on drift chambers, as well as their application in highenergy physics experiments, the reader is urged to consult [34][35][36].

\subsubsection{Vertex Drift Chamber}

The Vertex Drift Chamber (VTX) [37][38][39] is the innermost of D $\varnothing$ 's tracking chambers. The design specifications were to provide precise position determination of interaction vertices. It is composed of three concentric and cylindrical layers occupying the region $3.7 \mathrm{~cm}<r<16.2 \mathrm{~cm}$. The inner layer measures $97 \mathrm{~cm}$ in length, with each successive layer being about $10 \mathrm{~cm}$ longer. A cross sectional view of the VTX chamber is shown in Figure 2.4.

The innermost layer of the VTX chamber consists of 16 azimuthal cells, while the outer two of 32 cells each. Each cell has eight $25 \mu \mathrm{m}$ nickel-cobalt-tin (NiCoSn) sense wires, which are staggered by $\pm 100 \mu \mathrm{m}$ azimuthally in order to resolve left/right 


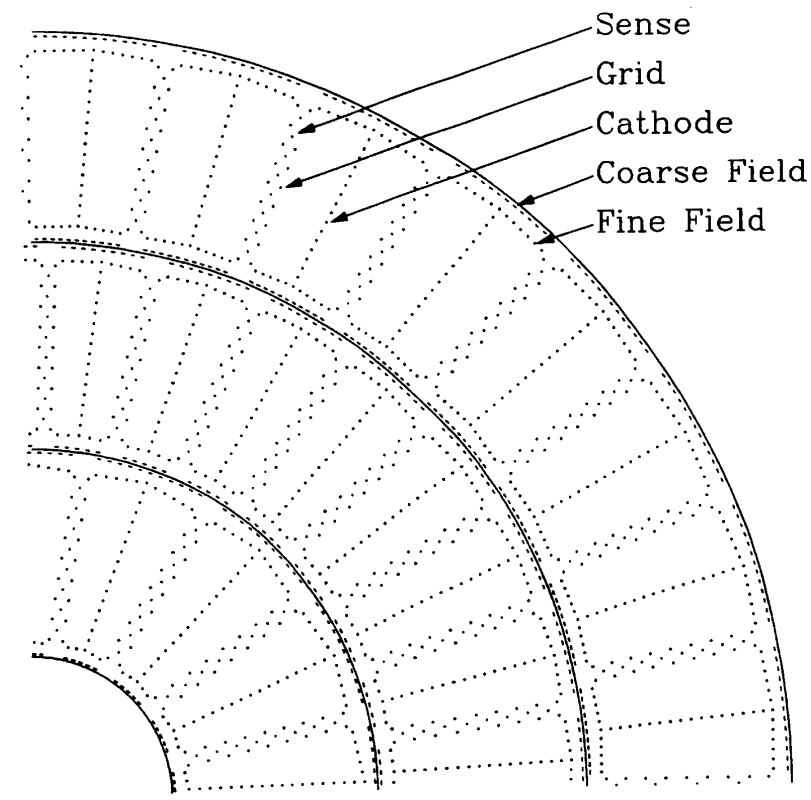

Figure 2.4: Cross sectional view of the VTX chamber.

Table 2.1: Vertex Chamber parameters.

\begin{tabular}{lc}
\hline \hline Number of layers & 3 \\
Active radius & $3.7-16.2 \mathrm{~cm}$ \\
Active length/layer & $96.6 \mathrm{~cm}, 106.6 \mathrm{~cm}, 116.8 \mathrm{~cm}$ \\
Num. of cells/layer & $16,32,32$ \\
Num. sense wires & $8 /$ cell, 640 total \\
Sense wire separation & $4.57 \mathrm{~mm}$ radially with $\pm 100 \mu \mathrm{m}$ stagger \\
Sense wire diameter & $25 \mu \mathrm{m} \mathrm{NiCoSn}$ \\
Guard wire diameter & $152 \mu \mathrm{m} \mathrm{Au}-\mathrm{plated} \mathrm{Al}$ \\
Sense wire potential & $+2.5 \mathrm{kV}$ \\
Gas mixture & $\mathrm{CO}_{2}(95 \%)-\mathrm{C}_{2} \mathrm{H}_{6}(5 \%)$ \\
Gas pressure & $1 \mathrm{~atm}$ \\
Drift field & $1.0-1.6 \mathrm{kV} / \mathrm{cm}$ \\
Average drift velocity & $7.3-12.8 \mu \mathrm{m} / \mathrm{ns}$ \\
Gas gain at sense wires & $4 \times 10^{4}$ \\
\hline \hline
\end{tabular}


ambiguity (this will be further explained in Section 3.1). Further enhancement of pattern recognition is accomplished by offsetting the cells azimuthally in each layer. The $r \phi$ coordinate measurement of a hit is achieved from the drift time and the wire hit, while the $z$ position is inferred via charge division: the sense wire is read out at both ends and is treated as a voltage divider. Unfortunately, this method is not very well suited for a high luminosity environment, since it requires that the sense wire pulses be well separated and that the cell have low occupancy. The $r \phi$ and $z$ coordinate spatial resolutions are approximately $60 \mu \mathrm{m}$ and $1.5 \mathrm{~cm}$ respectively. Further parameters of, and a summary of the VTX design specifications, are listed in Table 2.1.

\subsubsection{Transition Radiation Detector}

The Transition Radiation Detector (TRD) [40][41] occupies the space just beyond the radial extent of the VTX. The physical principle behind the TRD is the emission of transition X-rays by highly relativistic charged particles when they cross the boundary separating media with different dielectric constants. The emission of radiation occurs in the forward direction along the path of the charged particle $[36$, p. 136]. The intensity of the radiation is proportional to the relativistic gamma factor, $\gamma=E /\left(m c^{2}\right)$, and is contained within a cone whose opening angle is $2 / \gamma$. Making use of these two characteristics allows one to distinguish between particles of similar energies but differing masses. In practice, the X-ray radiation emitted from a particle crossing a single boundary is not readily detectable, so one stacks a large 


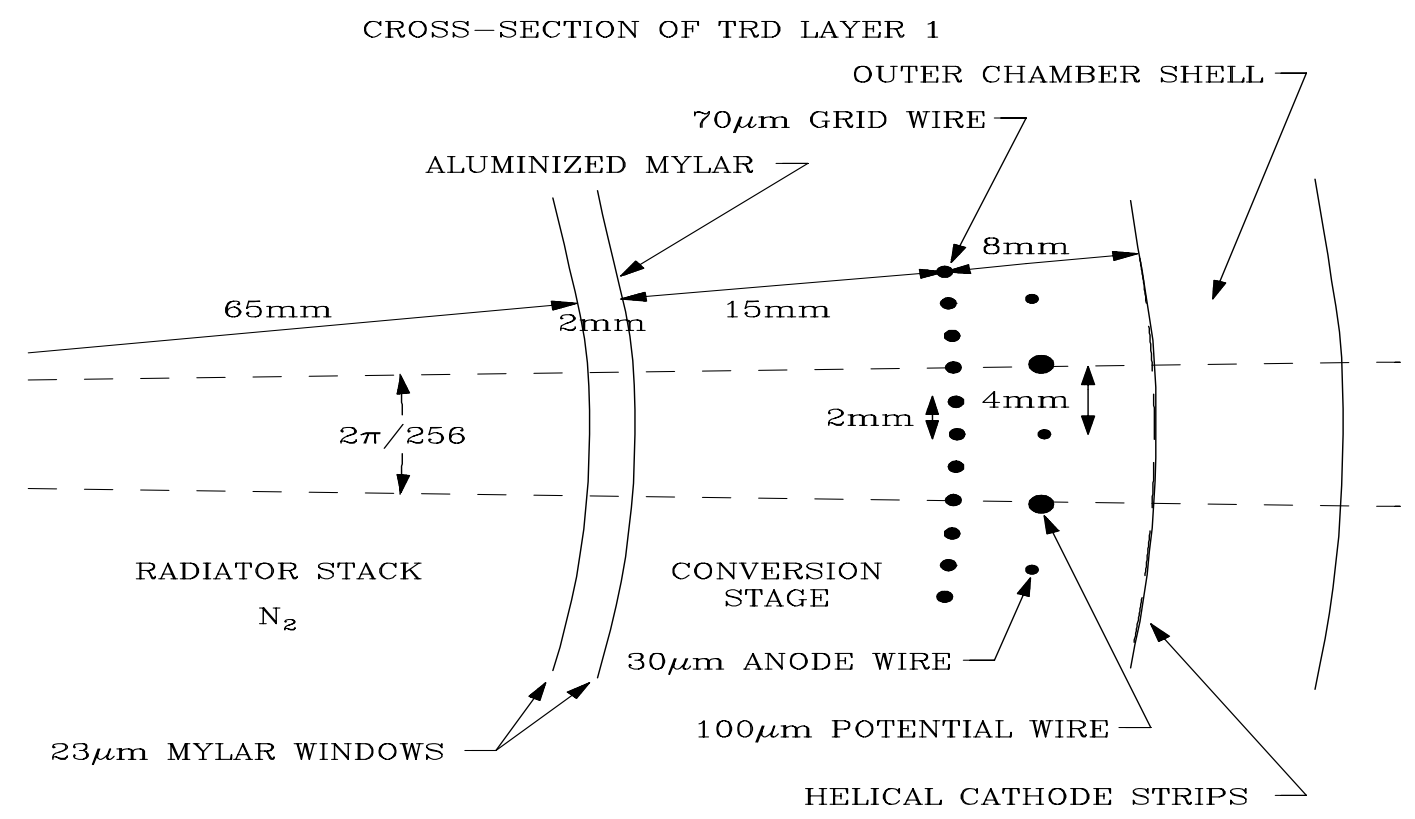

Figure 2.5: Cross sectional view of the first TRD layer.

number of such boundaries in order to obtain a measurable signal. The X-rays are in turn detected when they ionize the gas that is present in the TRD. The magnitude and arrival time of charged clusters are then used to distinguish between different particles.

The TRD at $\mathrm{D} \varnothing$ is primarily used in electron identification: its responsibility is the distinction of electrons from the heavier hadrons. Similar to the VTX, it consists of three concentric, cylindrical layers. Each layer has a radiator and an Xray detection chamber. The radiator consists of 393, $18 \mu$ m thick, polypropylene foils separated by a mean gap of $150 \mu \mathrm{m}$. These gaps are filled with nitrogen $\left(\mathrm{N}_{2}\right)$ gas. The $\mathrm{X}$-ray detector is a two stage time-expansion radial-drift proportional wire chamber mounted after the radiator. It is composed of a $15 \mathrm{~mm}$ conversion and an $8 \mathrm{~mm}$ amplification stages separated by a cathode grid of $70 \mu \mathrm{m}$ gold-plated tungsten (W) wire. The radiator and detector volumes are separated by a pair of $23 \mu \mathrm{m}$ windows 
where dry $\mathrm{CO}_{2}$ gas flows to prevent the $\mathrm{N}_{2}$ gas in the radiator from leaking into the detector volume and polluting the xenon $(\mathrm{Xe})$, methane $\left(\mathrm{CH}_{4}\right)$, ethane $\left(\mathrm{C}_{2} \mathrm{H}_{6}\right)$ chamber gas mixture $(91 \%: 7 \%: 2 \%)$. The TRD was designed to achieve a $10^{4}$ rejection factor against pions while remaining $90 \%$ efficient with isolated electrons. However, for efficiency reasons, the TRD is not used in this analysis.

\subsubsection{Central Drift Chamber}

The outermost tracker is the Central Drift Chamber (CDC) [38][42][43][44] consisting of four cylindrical, concentric layers. The CDC covers the pseudorapidity range $|\eta|<1.2$, providing the trajectory and ionization information on isolated charged particles. The four layers, shown in Figure 2.6, occupy the radial region between 49.5 $\mathrm{cm}$ and $74.5 \mathrm{~cm}$, while measuring $184 \mathrm{~cm}$ in length.

Each of the CDC layers is divided into 32 modular, azimuthal cells. Each cell contains seven $30 \mu \mathrm{m}$ gold-plated tungsten sense wires, and two delay lines. As in the case of the VTX, the sense wires are staggered by $\pm 200 \mu \mathrm{m}$ azimuthally to remove the left/right ambiguity, and alternate cells are offset azimuthally by half of a cell to enhance pattern recognition. The $r \phi$ coordinate measurement of a hit is achieved from the drift time and the wire hit. The $z$ position is inferred via the use of the delay lines: these are inductive wires that transmit an induced pulse whenever an avalanche occurs near outer sense wires. By looking at the difference in arrival times at both ends of the delay line, one can estimate where on the delay line the 


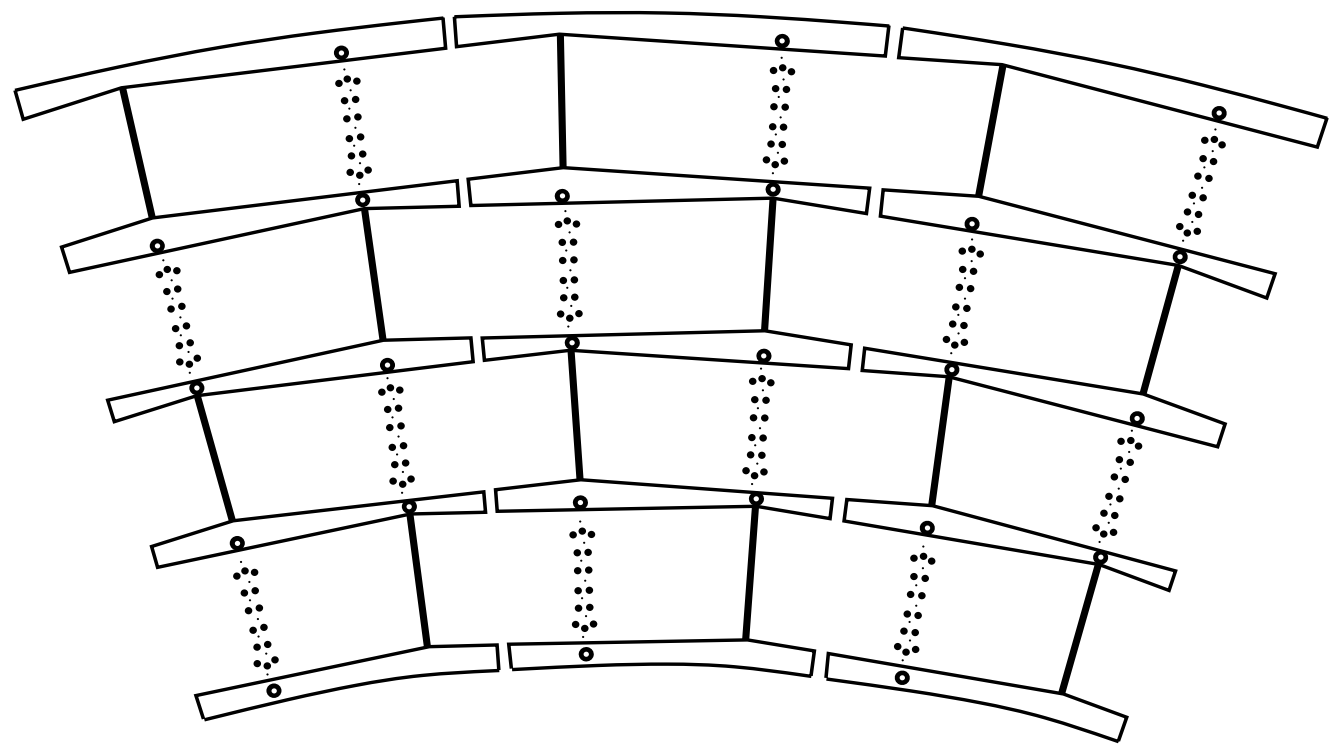

Figure 2.6: Cross sectional view of the CDC.

Table 2.2: Central Drift Chamber parameters.

\begin{tabular}{lc}
\hline \hline Length of active volume & $179.4 \mathrm{~cm}$ \\
Active radius & $51.8-71.9 \mathrm{~cm}$ \\
Number of layers & 4 \\
Num. of cells/layer & 32 \\
Num. of sense wires & $7 /$ cell, 896 total \\
Num. of delay lines & $2 /$ cell, 256 total \\
Sense wire separation & $6 \mathrm{~mm}$ with $\pm 200 \mu \mathrm{m}$ stagger \\
Sense wire diameter & $30 \mu \mathrm{m} \mathrm{Au-plated} \mathrm{W}$ \\
Guard wire diameter & $125 \mu \mathrm{m} \mathrm{Au}-\mathrm{plated} \mathrm{CuBe}$ \\
Sense wire potential & $+1.5 \mathrm{kV}$ \\
Gas mixture & $\mathrm{Ar}(93 \%)-\mathrm{CH} \mathrm{H}_{4}(4 \%)-\mathrm{CO}_{2}(3 \%)$ \\
Gas pressure & $1 \mathrm{~atm}$ \\
Drift field & $620 \mathrm{~V} / \mathrm{cm}$ \\
Average drift velocity & $34 \mu \mathrm{m} / \mathrm{ns}$ \\
Gas gain at sense wires & $2.6 \times 10^{4}$ \\
\hline \hline
\end{tabular}


hit actually occurred. The $r \phi$ and $z$ coordinate spatial resolutions are approximately $180 \mu \mathrm{m}$ and $2.9 \mathrm{~mm}$ respectively. Further parameters of, and a summary of the CDC design specifications, are listed in Table 2.2.

\subsubsection{Forward Drift Chambers}

The Forward Drift Chambers (FDC) [38][42][45][46], located at both ends of the CD system, extend the outer tracking coverage down to an angle of $\theta \approx 5^{\circ}$, or a pseudorapidity $\eta \approx 3.1$. These two sets of chambers are themselves composed of three chambers: the $\Phi$ chamber (with sense wires oriented axially to measure the $\phi$ coordinate of the hits) is sandwiched between two $\Theta$ chambers. Each $\Theta$ chamber is made of four quadrants (with sense wires oriented parallel to the $x$-axis for the top/bottom sub-chambers, and parallel to the $y$-axis for the left/right sub-chambers, to measure the $\theta$ coordinate of the hits). Figure 2.7 illustrates the layout of the FDC.

A $\Phi$ chamber is made up of 36 azimuthal cells, each containing 16 axial sense wires of length $50 \mathrm{~cm}$ that are parallel to the beam pipe. Each of the four quadrants of a $\Theta$ chamber contain six rectangular cells, located at increasing radii. Each cell contains eight sense wires and one delay line. Similar to the CDC, the sense wires in both $\Phi$ and $\Theta$ chambers are staggered by $\pm 200 \mu \mathrm{m}$ to help resolve left/right ambiguity. In addition, the two $\Theta$ chambers are rotated in $\phi$ by $45^{\circ}$ to aid in pattern recognition. The spatial resolutions are $200 \mu \mathrm{m}$ and $300 \mu \mathrm{m}$ for the $\Phi$ and $\Theta$ chambers 


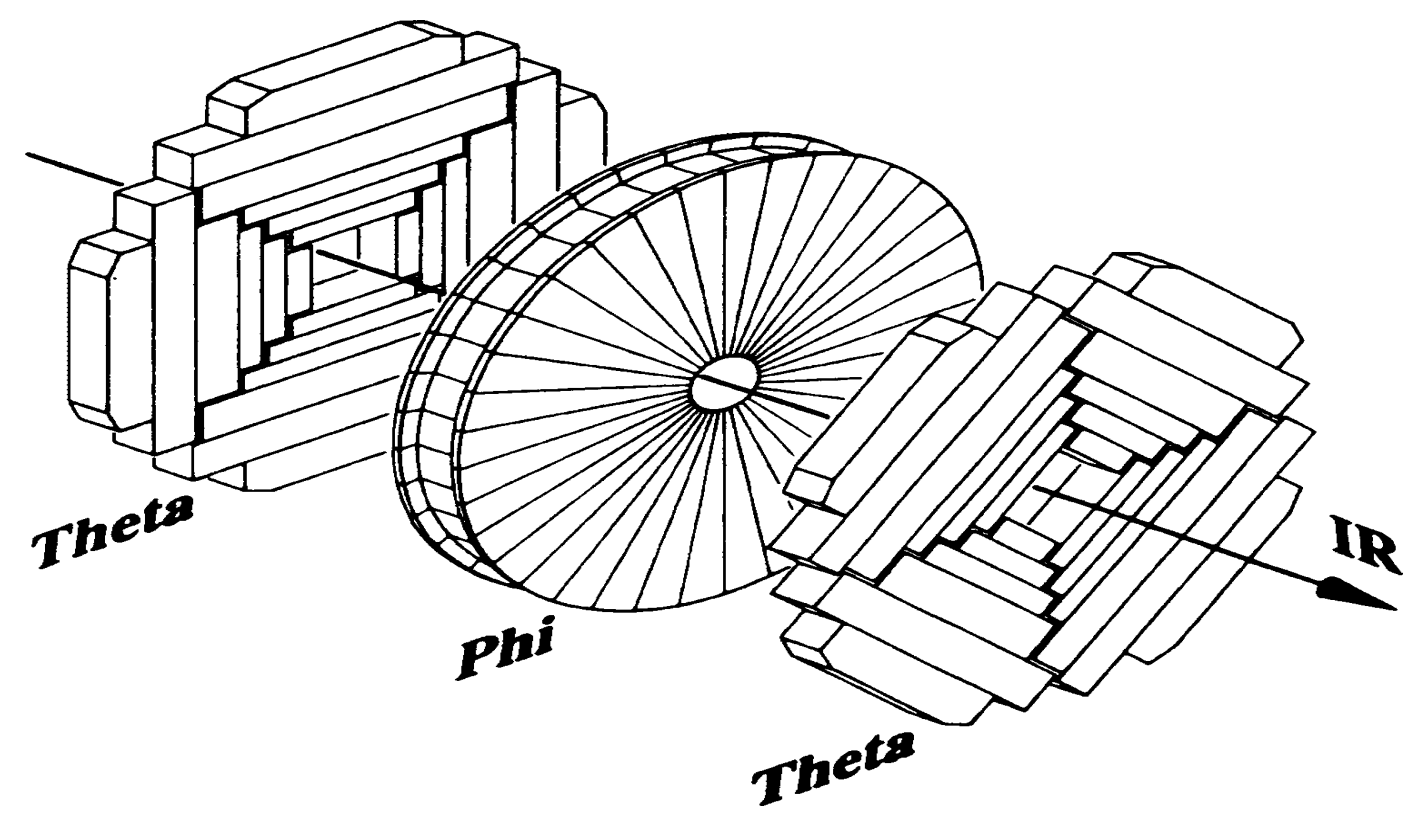

Figure 2.7: Layout of the FDC.

Table 2.3: Forward Drift Chamber parameters.

\begin{tabular}{lcc}
\hline \hline & $\Theta$ modules & $\Phi$ modules \\
\hline Active $z$ interval & $104.8-111.2 \mathrm{~cm}$ & $113.0-127.0 \mathrm{~cm}$ \\
& $128.8-135.2 \mathrm{~cm}$ & \\
Active radius & $11-62 \mathrm{~cm}$ & $11-61.3 \mathrm{~cm}$ \\
Number of modules & 4 & 2 \\
Num. of cells/module & $6 \times 4$ quadrants & 32 \\
Num. of sense wires/module & $8 /$ cell, 384 total & $16 /$ cell, 576 total \\
Num. of delay lines/module & $1 /$ cell, 48 total & - \\
Sense wire separation & $8 \mathrm{~mm}$ with $\pm 200 \mu \mathrm{m} \mathrm{stagger}$ \\
Sense wire diameter & \multicolumn{2}{c}{$30 \mu \mathrm{m} \mathrm{NiCoSn}$} \\
Guard wire diameter & \multicolumn{2}{c}{$163 \mu \mathrm{m} \mathrm{Au-plated} \mathrm{Al}$} \\
Sense wire potential & \multicolumn{2}{c}{$+1.5 \mathrm{kV}$} \\
Gas mixture & Ar $(93 \%)-\mathrm{CH}(4 \%)-\mathrm{CO}(3 \%)$ \\
Gas pressure & \multicolumn{2}{c}{1 atm } \\
Drift field & \multicolumn{2}{c}{$1.0 \mathrm{kV} / \mathrm{cm}$} \\
Average drift velocity & $37 \mu \mathrm{m} / \mathrm{ns} \quad 40 \mu \mathrm{m} / \mathrm{ns}$ \\
Gas gain at sense wire & $2.3,5.3 \times 10^{4}$ & $3.6 \times 10^{4}$ \\
\hline \hline
\end{tabular}


respectively. Further parameters of, and a summary of the FDC design specifications, are listed in Table 2.3.

\subsubsection{Central Detector Readout}

The electronics for reading out the signals of all CD devices are almost the same. Three stages of signal processing are performed. The first consists in leading the signals from the chamber wires into preamplifiers mounted directly on the chambers themselves. From there, these signals are fed into analog pulse shaping cards located on the support platform underneath the detector. Finally, the signals are sent to Flash Analog-To-Digital (FADC) converters located in the $\mathrm{MCH}$, where the signals are sampled and digitized at the rate of $106 \mathrm{MHz}$ (starting with the beam crossing). If the event is not accepted by the Level-1 trigger (see Section 2.7.2), the data is overwritten by the next crossing. Otherwise, the data is compressed by eliminating the flat portions of the signal between the pulses, a process known as zero suppression, and sent on to the Level-2 trigger.

\subsection{Calorimetry}

The Calorimeter system at $\mathrm{D} \varnothing$ is responsible for measuring the energy of most particles that are incident on it. It is the crucial part of the D $\varnothing$ detector since it provides the only means to measure the energy of electrons, photons, and jets. 
Furthermore, it plays a vital role in the identification of all final state particles: leptons, photons, jets, and neutrinos.

The interaction of photons and electrons with matter at energies well above 10 $\mathrm{MeV}$ occurs primarily via the creation of electron/positron pairs and the Bremsstrahlung mechanism ${ }^{4}$. An electromagnetic shower develops as an alternating sequence of interactions of these two types. For example, a primary electron will lose energy by emitting a photon. The photon will convert into an $\mathrm{e}^{+} \mathrm{e}^{-}$pair, which in turn will lose energy by emitting other photons. The process keeps occurring and the shower keeps developing until the energy of all secondary particles reaches the level where ionization losses and atomic excitations become important. Since, at high energies, the angles of emission of the electrons and photons are small, the shower develops primarily in the direction of motion of the original electron. The energy loss of an electromagnetic particle is characterized by the radiation length $X_{\circ}$ :

$$
\frac{d E}{E}=-\frac{d x}{X_{\circ}}
$$

The radiation length is dependent on the absorbing medium ${ }^{5}$ : it is $0.32 \mathrm{~cm}$ for uranium and $13.5 \mathrm{~cm}$ for liquid argon. In a mixture or compound, it is approximated by:

$$
1 / X_{\circ}=\sum w_{j} / X_{j}
$$

where $w_{j}$ and $X_{j}$ are the fraction by weight and the radiation length of the $j$ th element in the mixture.

\footnotetext{
${ }^{4}$ This is the mechanism where a charged particle interacts with the Coulomb field surrounding a nucleus and emits an energetic photon.

${ }^{5}$ The radiation length of a material of atomic number $A$, and charge $Z$ can be approximated [47] as $X_{\circ}\left[\mathrm{g} / \mathrm{cm}^{2}\right]=180 A / Z^{2}$.
} 
The physical process governing the interaction of a hadronic particle with matter is quite different than the one just described for electromagnetic particles. Hadronic showers are produced from the inelastic collisions of hadrons with the surrounding atomic nuclei, or from the multi-particle production of slow pions and kaons. These secondary hadrons will in turn undergo additional inelastic collisions, or produce more slow hadrons. Shower development ceases once ionization losses and nuclear absorption become dominant. It is important to note that typical secondary hadron production occurs with a transverse momentum of $\sim 350 \mathrm{MeV} / c$ [47]. Hence, hadronic showers tend to be more spread out laterally than electromagnetic ones. The longitudinal development of the hadronic showers scales with the nuclear absorption (or interaction) length of the medium, $\lambda_{\circ}$. The absorption length for uranium is $\lambda_{\circ}=10.5 \mathrm{~cm}$, thus causing the hadronic shower to be much larger than an electromagnetic shower of similar energy.

Although a homogeneous calorimeter ${ }^{6}$ achieves better resolutions, it is far more practical in large, high-energy detectors to use a sampling calorimeter. In a sampling calorimeter, the shower development of an incident particle is periodically sampled in sensitive layers via the ionization of the active medium (such as liquid argon) or the use of a scintillator. The sampling layers are interspersed with layers of passive absorber, thus making it possible to build rather compact devices. Since most of the energy ends up being absorbed by the passive material, only a small fraction is read

\footnotetext{
${ }^{6} \mathrm{~A}$ homogeneous calorimeter is made of one continuous absorber, usually using some inorganic scintillating crystal (such as NaI, BGO or lead glass).
} 
out, and a correction factor which is proportional to the sampling fraction:

$$
s f=\frac{E_{\text {active }}}{E_{\text {active }}+E_{\text {passive }}}
$$

is needed to obtain the total energy loss by the incident particle. Typically, $5-10 \%$ of an incident particle's energy gets deposited in the active layers through the ionization of the liquid argon.

Another desirable aspect in calorimetry is compensation: making the calorimeter's response to electromagnetic and hadronic showers as close as possible. The ratio of the two responses is known as the $e / h$ ratio, and is not necessarily equal to one since the energy response of hadronic showers tends to be smaller [47] than electromagnetic showers ${ }^{7}$. However, an $e / h$ ratio that is close to unity is achieved by either decreasing the electron response, or by boosting the hadronic signal. The latter can be achieved by using uranium-238 or thorium as the absorber. In $\mathrm{U}^{238}$, for example, some of the normally invisible energy lost in breaking up nuclei induces fission, which in turn emits detectable energy in the calorimeter.

It should be emphasized that an $e / h$ ratio that is close to one is important, since it leads to an improvement in the energy resolution. This is caused by the fact that a hadronic shower contains an electromagnetic component, such as photons produced in $\pi^{0}$ and $\eta$ decays. This electromagnetic component can vary largely from shower to shower, but if the $e / h$ ratio is one, the energy resolution will not be affected.

\footnotetext{
${ }^{7}$ In hadronic showers, a considerable fraction of the energy, such as nuclear binding energy, muons, and neutrinos, is 'invisible' since it escapes detection, or is not able to reach the active layers.
} 
The energy resolution of a sampling calorimeter can still be degraded even if the calorimeter is perfectly compensating. The energy resolution is determined by the fluctuations in the shower development. Since this is a statistical process in nature, one expects the fractional error to scale as $1 / \sqrt{E}$. Additional sources of energy fluctuations in the calorimeter could include [35, p. 270]:

- Actual energy deposited in the active layers (sampling fluctuations);

- Energy leakage out of the calorimeter with longitudinal losses causing a larger degradation than lateral ones;

- Variations in the construction or control of the calorimeter modules, such as absorber thickness and spacing;

- Noise in the active layers (due to the natural decay of the uranium-238);

- Electronic noise;

- Pile-up in the calorimeter (more than one event within the time resolution).

The calorimeter can also used to measure the positions of incident particles. This is achieved by segmenting the calorimeter, thus enabling one to measure the position and angle of the incident particles by studying their shower development. The impact point of the incident particle is determined by probing the shower in the early part of its development. Very precise determination of the position is also achieved by studying the transverse shower profile at the shower maximum and calculating its center of gravity. The shower maximum is the depth along the electromagnetic 
shower where the maximum number of particles are present in the shower [35, $\mathrm{p}$. 162]. The position resolution, similar to the energy resolution, depends on the fluctu-

ations within the shower: it scales as $1 / \sqrt{E}$. It also depends on the segmentation of the calorimeter: the resolution increases with the number of cells hit by the shower particles, and decreases with the cell size [36, p. 157].

For further discussions on calorimeters, the reader is referred to [35][36], as well as the excellent articles [47][48].

\subsubsection{Calorimeter Design}

The DØ Calorimeter is a sampling calorimeter, with liquid argon (LAr) as the active medium to sample the ionization produced in electromagnetic or hadronic showers. The use of LAr requires a containment vessel (cryostat), where the argon is kept cold enough to remain in the liquid form. In order to retain access to the central tracking detectors, the chosen design, shown in Figure 2.8, consisted of one Central Cryostat (CC) covering the region $|\eta|<1.2$, two Endcap Cryostats (EC) extending the coverage to $|\eta| \approx 4$, and the Inter-cryostat Detector (ICD) to cover their overlapping region.

The DØ Calorimeter is highly modular, and finely segmented in the transverse and longitudinal shower directions. Three distinct types of modules are used in the CC and EC: an electromagnetic section (EM) with relatively thin uranium-238 absorber plates, a fine hadronic section $(\mathrm{FH})$ with thicker uranium plates and a coarse hadronic 


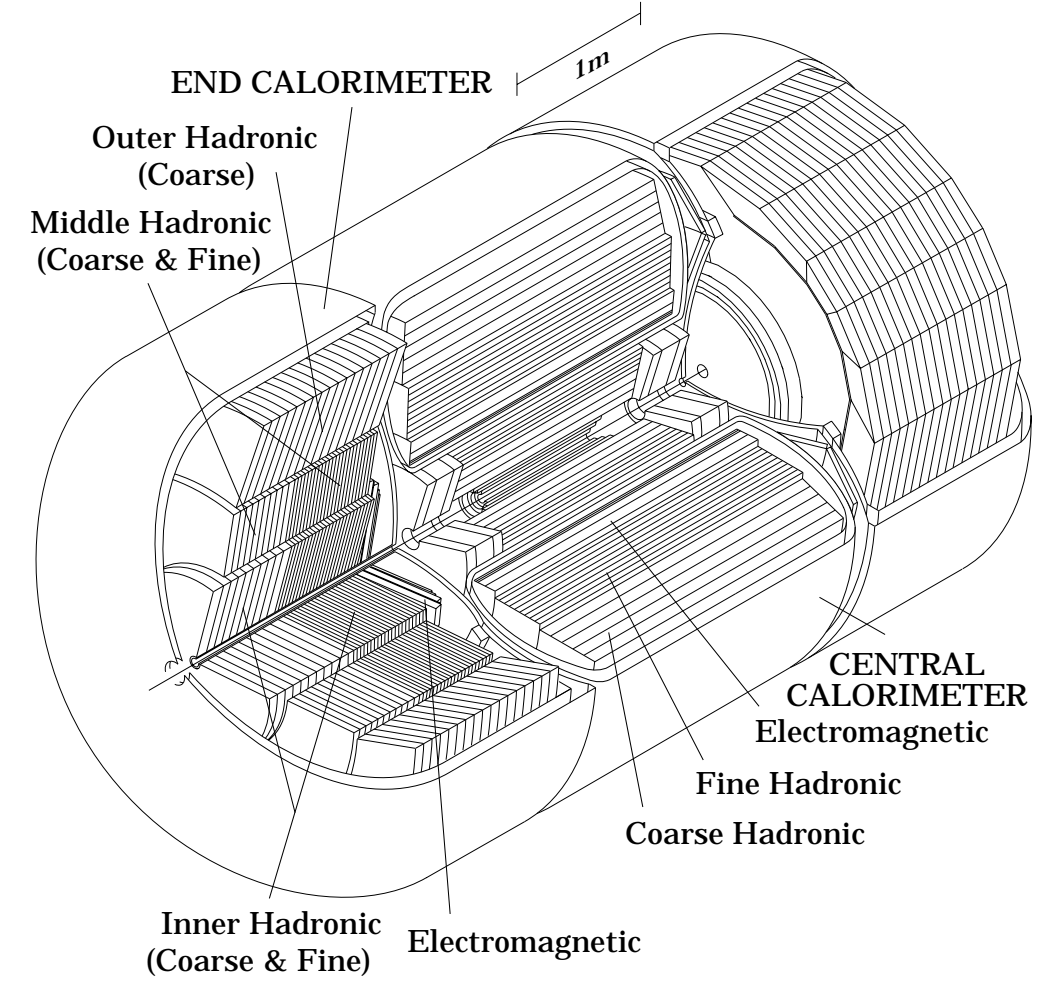

Figure 2.8: Isometric view of the DØ calorimeter system.

section $(\mathrm{CH})$ with thick copper or stainless steel plates. Each module consists of a row of alternating absorber plates and signal readout boards, as shown in Figure 2.9. The $2.3 \mathrm{~mm}$ gap separating adjacent absorber plates and signal boards is filled with LAr. The signal boards consist of a copper pad with two separate $0.5 \mathrm{~mm}$ thick G-10 sheets laminated at each end. The outer surfaces of the boards were coated with a highly resistive epoxy. An electric field is established by grounding the absorber plate while applying a positive potential (typically $2.0-2.5 \mathrm{kV}$ ) to the resistive surfaces of the signal boards. Incident particles shower in the absorber plates, and the resulting shower particles ionize the LAr in the adjacent gap. The liberated electrons drift to the signal boards (the drift time is $\approx 450 \mathrm{~ns}$ ), and induce a signal on the copper pad. 
Signals from several signal boards in the same $\eta$ and $\phi$ region are ganged together in depth to form a readout cell.

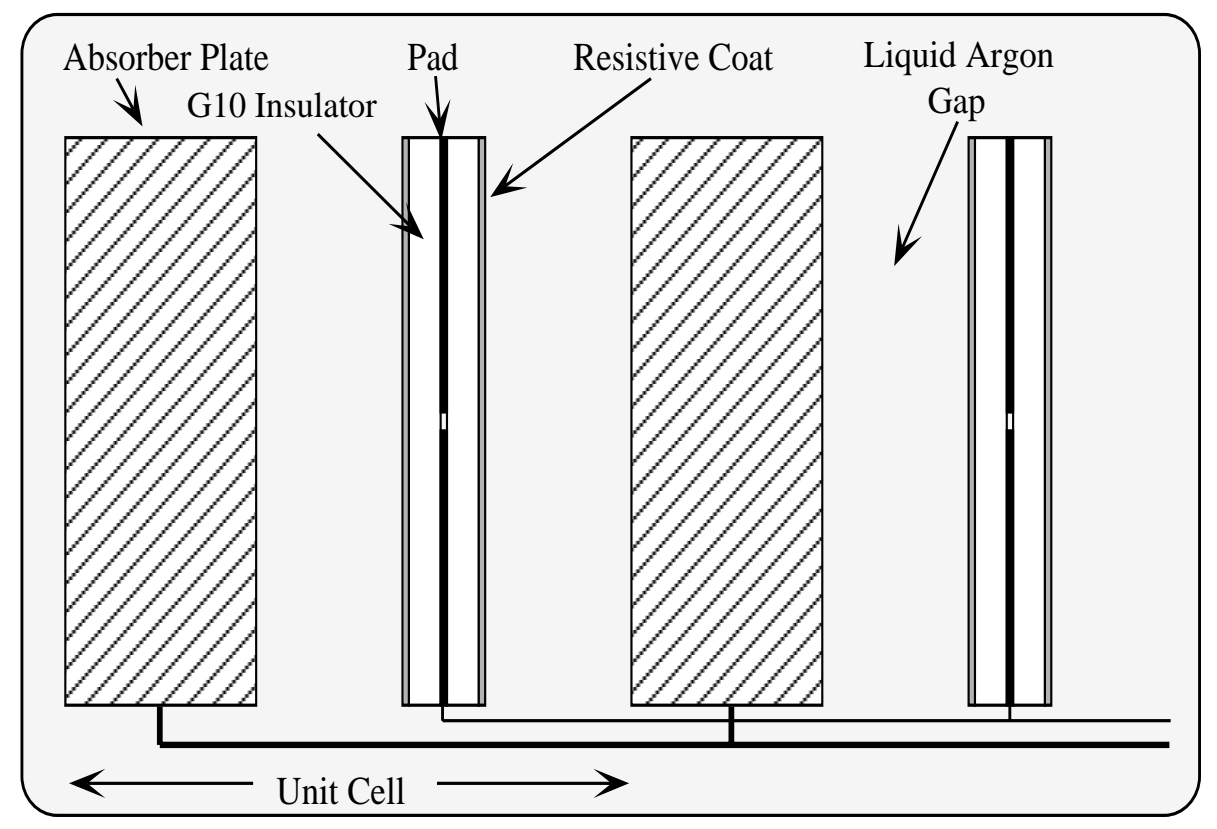

Figure 2.9: Schematic view of a $D \varnothing$ calorimeter cell.

The pattern and sizes of the readout cells were determined from considerations of shower sizes. The transverse dimensions of the readout cell were chosen to be similar to the transverse sizes of showers: $\sim 1-2 \mathrm{~cm}$ for EM showers and $\sim 10 \mathrm{~cm}$ for hadronic showers. Furthermore, longitudinal segmentation within the EM, FH and $\mathrm{CH}$ layers helps in the distinction and separation of electrons from hadrons. The design was chosen to be pseudo-projective: the centers of the cells lie on lines which project back to the center of the detector, but the cell boundaries are aligned perpendicular to the absorber plates. This is clearly illustrated in Figure 2.10. 


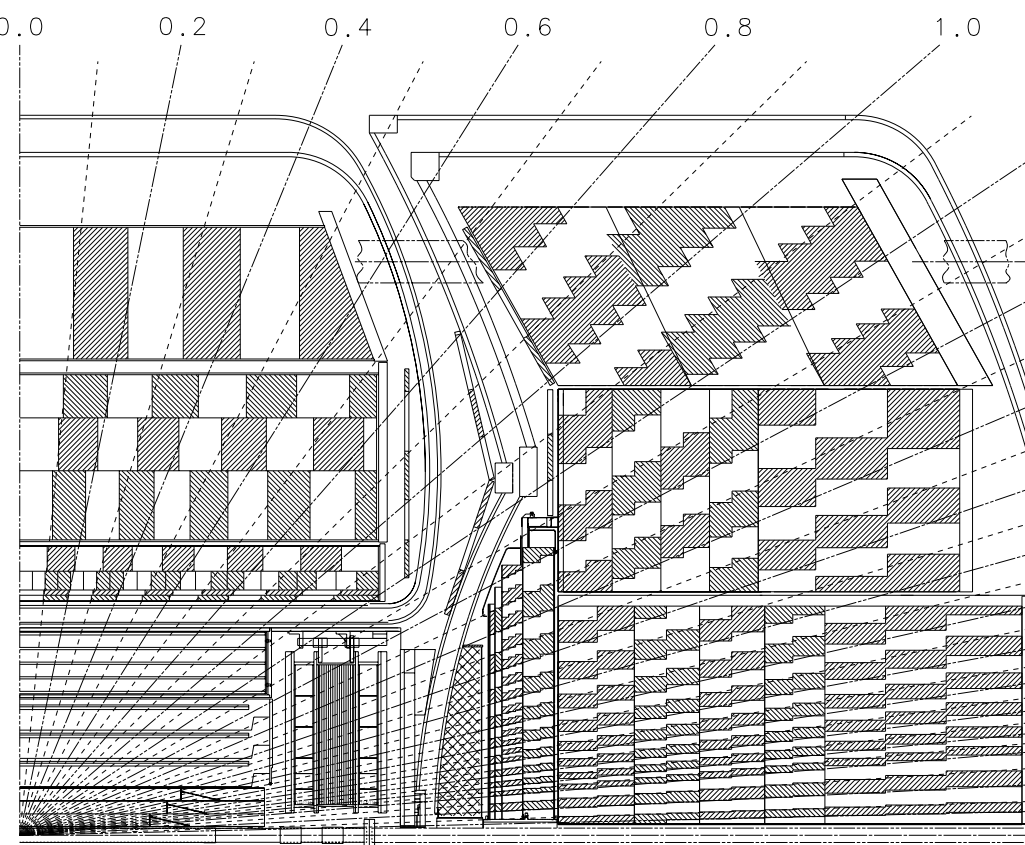

Figure 2.10: Side view of one quadrant of the calorimeters. Also shown are lines of constant pseudorapidity intervals.

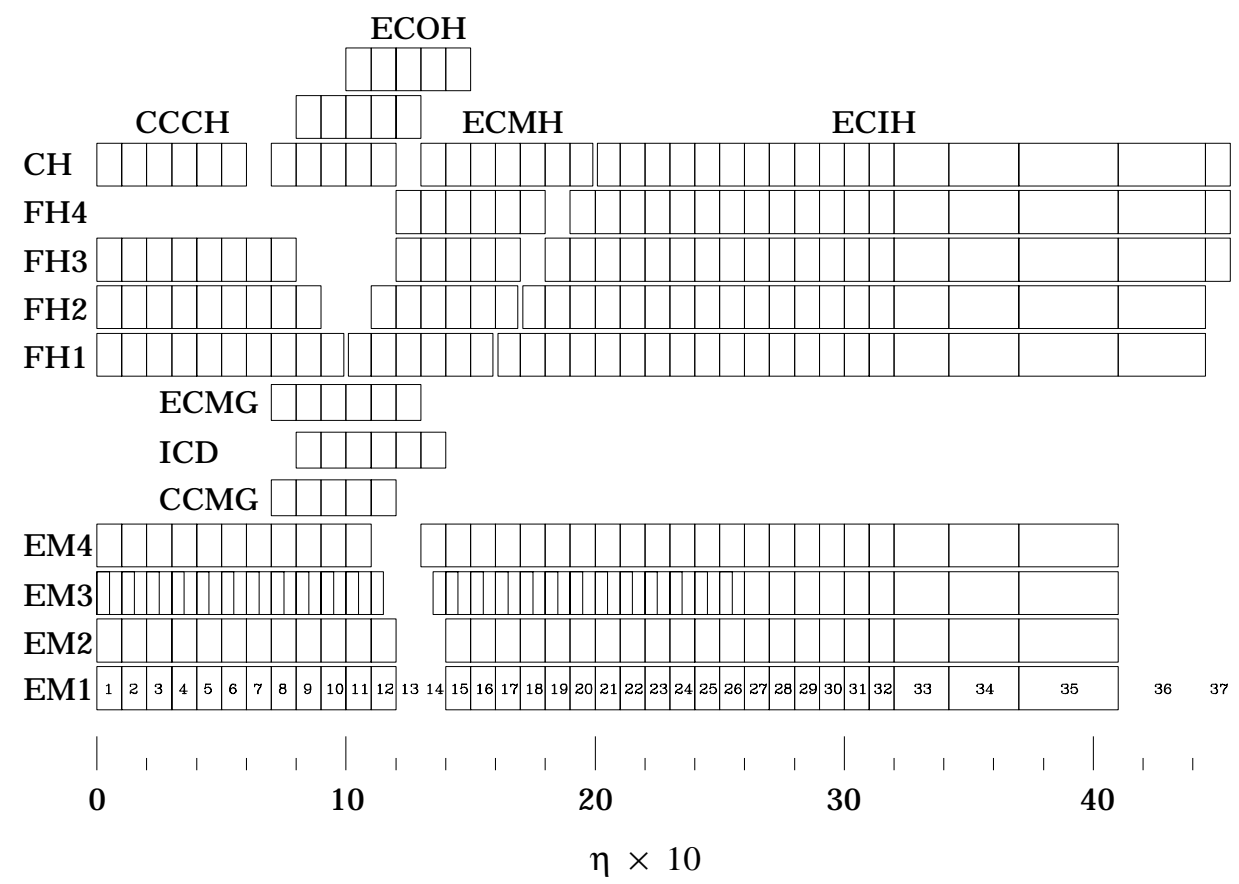

Figure 2.11: Segmentation of the D $\varnothing$ calorimeter towers. 


\subsubsection{Central Calorimeter}

The Central Calorimeter (CC) is composed of three cylindrical concentric shells parallel to the beam axis. Radially, it occupies the space $75<r<222 \mathrm{~cm}$ from the beam pipe with a length of $226 \mathrm{~cm}$, thus achieving an angular coverage of $35^{\circ}<\theta<$ $145^{\circ}$, or $|\eta|<1.2$. The inner shell consists of 32 electromagnetic (EM) modules, thick enough to contain most electromagnetic showers. The middle shell, made of 16 fine hadronic $(\mathrm{FH})$ modules, measures showers of hadronic particles, while the outer layer, made of 16 coarse hadronic $(\mathrm{CH})$ modules, measures any leakage out of the FH layer while minimizing punchthrough, the energy flow out of the calorimeter and into the muon system. The EM modules consist of 21 radial cells, arranged in four readout layers (EM1 through EM4). Each cell is composed of a $3 \mathrm{~mm}$ depleted uranium absorber plate and a $2.3 \mathrm{~mm}$ LAr gap for a sampling fraction of $12.9 \%$. The FH modules consist of 50 radial cells, arranged in three readout layers (FH1 through FH3), with each cell made from a $6 \mathrm{~mm}$ uranium-niobium alloy (U-Nb) absorber plate and a $2.3 \mathrm{~mm}$ LAr gap for a sampling fraction of $6.9 \%$. Finally, the $\mathrm{CH}$ modules consist of 9 radial cells, but only one readout layer. The $\mathrm{CH}$ cells use $4.75 \mathrm{~cm}$ copper absorber plates with a $2.3 \mathrm{~mm}$ LAr gap for a sampling fraction of $1.7 \%$.

The transverse segmentation of the calorimeter is $0.1 \times 0.1$ in $\eta \times \phi$ space, except in the third EM layer (EM3). This layer, corresponding to the EM shower maximum, has its segmentation increased to $0.05 \times 0.05$ in $\eta \times \phi$ space in order to fully optimize the distinguishability between electron and hadronic showers. In addition, 
each concentric shell (EM, FH and $\mathrm{CH}$ ) is rotated azimuthally, thus avoiding any continuous cracks.

The segmentation of the calorimeter is shown in Figure 2.11, while the major design specifications of the CC are listed in Table 2.4.

Table 2.4: Central Calorimeter parameters.

\begin{tabular}{lccc}
\hline \hline CC module type & EM & FH & CH \\
\hline Rapidity coverage & \pm 1.2 & \pm 1.0 & \pm 0.6 \\
Number of modules & 32 & 16 & 16 \\
Absorber & Uranium & Uranium & Copper \\
Absorber thickness [cm] & 0.3 & 0.6 & 4.65 \\
Liquid argon gap [cm] & 0.23 & 0.23 & 0.23 \\
Number of cells per module & 21 & 50 & 9 \\
Longitudinal depth & $20.5 X_{\circ}$ & $3.24 \lambda_{\circ}$ & $2.93 \lambda_{\circ}$ \\
Number of readout layers & 4 & 3 & 1 \\
Cells per readout layer & $2,2,7,10$ & $21,16,13$ & 9 \\
Total radiation lengths & 20.5 & 96.0 & 32.9 \\
Radiation length per cell & 0.975 & 1.92 & 3.29 \\
Total absorption lengths $(\lambda)$ & 0.76 & 3.2 & 3.2 \\
Absorption length per cell & 0.036 & 0.0645 & 0.317 \\
Sampling fraction [\%] & 11.79 & 6.79 & 1.45 \\
Segmentation $(\phi \times \eta)^{b}$ & $0.1 \times 0.1$ & $0.1 \times 0.1$ & $0.1 \times 0.1$ \\
Total number of readout cells & 10,368 & 3456 & 768 \\
\hline \hline
\end{tabular}

${ }^{a}$ Uranium is depleted and FH absorbers contain $1.7 \%$ Niobium alloy

${ }^{b}$ EM3 layer has $0.05 \times 0.05$

\subsubsection{Endcap Calorimeters}

The two Endcap Calorimeters (EC) provide coverage on either side of the CC from a pseudorapidity of 1.3 out to about 4 . This corresponds to an angular coverage of $2^{\circ}<\theta<30^{\circ}$, and $150^{\circ}<\theta<178^{\circ}$. Each EC cryostat is divided into four sections: the electromagnetic (EM), the inner hadronic (IH), the middle hadronic (MH), and 
the outer hadronic $(\mathrm{OH})$. The EM modules in the EC (EMEC) are disk shaped and occupy the center of the EC cryostat. The radial coverage starts at $5.7 \mathrm{~cm}$ and extends to an outer radius varying between $84 \mathrm{~cm}$ to $104 \mathrm{~cm}$, corresponding to an angular coverage of $3^{\circ}<\theta<27^{\circ}$. The modules consist of 18 radial cells with absorber plates made from $4 \mathrm{~mm}$ thick depleted uranium. The cells are arranged into four readout layers (EM1 through EM4). The transverse segmentation is mostly $0.1 \times 0.1$ in $\eta \times \phi$ space; however, for $|\eta|>3.2$, the pad size becomes too small so the segmentation is increased to $0.2 \times 0.2$. As in the CC, the third ECEM layer has finer segmentation to improve electron/hadron shower resolution. The segmentation is $\Delta \eta \times \Delta \phi=0.05 \times 0.05$ for $|\eta|<2.7,0.1 \times 0.1$ for $2.7<|\eta|<3.2$, and $0.2 \times 0.2$ for $|\eta|>3.2$

The IH module, located directly behind the ECEM, is cylindrically shaped with inner and outer radii $3.92 \mathrm{~cm}$ and $86.4 \mathrm{~cm}$ respectively. Longitudinally, the $\mathrm{IH}$ is divided into fine hadronic (IFH) and coarse hadronic (ICH) sections. The IFH consists of 16 cells - each made from $6 \mathrm{~mm}$ thick semicircular uranium absorber plates - that are arranged in four readout layers (FH1 through FH4). In order to avoid cracks, each alternate plate is rotated by $90^{\circ}$ in $\phi$. The ICH consists of a single readout layer made from 13 cells, each using $46.5 \mathrm{~mm}$ stainless steel absorber plates. The IH transverse segmentation matches that of the ECEM: $\Delta \eta \times \Delta \phi=0.1 \times 0.1$ for $|\eta|<3.2$, and $0.2 \times 0.2$ otherwise; however, for $|\eta|>3.8$ (beyond the ECEM coverage), it is $0.4 \times 0.2$.

Surrounding the inner core of EM and IH modules in the EC is the MH ring. This ring, consisting of 16 wedge shaped modules, extends from an inner radius 
of $33 \mathrm{~cm}$ to an outer radius of $152 \mathrm{~cm}$. Like the $\mathrm{IH}$, each $\mathrm{MH}$ module is divided longitudinally into fine hadronic $(\mathrm{MFH})$ and coarse hadronic $(\mathrm{MCH})$ sections. The MFH consists of 60 radial cells arranged in four readout layers (FH1 through FH4). Each cell uses $6 \mathrm{~mm}$ U-Nb alloy absorber plates. The $\mathrm{MCH}$ is a single readout layer consisting of 14 cells. Each cell uses $46.5 \mathrm{~mm}$ stainless steel absorber plates. The transverse segmentation of the $\mathrm{MH}$ is exactly like the $\mathrm{IH}$.

Table 2.5: Endcap Calorimeter parameters.

\begin{tabular}{|c|c|c|c|c|c|c|}
\hline$\overline{\text { EC module type }}$ & $\overline{\mathrm{EM}}$ & $\overline{\mathrm{IFH}}$ & $\overline{\mathrm{ICH}}$ & $\overline{\mathrm{MFH}}$ & $\overline{\mathrm{MCH}}$ & $\overline{\mathrm{OH}}$ \\
\hline Rapidity coverage & $1.3-4.1$ & $1.6-4.5$ & $2.0-4.5$ & $1.0-1.7$ & $1.3-2.0$ & $0.7-1.4$ \\
\hline Num. of modules/cryostat & 1 & 1 & 1 & 16 & 16 & 16 \\
\hline Absorber $^{a}$ & $\mathrm{U}$ & $\mathrm{U}$ & $\mathrm{SS}^{b}$ & $\mathrm{U}$ & SS & SS \\
\hline Absorber thickness $[\mathrm{cm}]$ & 0.4 & 0.6 & 4.6 & 0.6 & 4.6 & 4.6 \\
\hline Liquid argon gap [cm] & 0.23 & 0.21 & 0.21 & 0.22 & 0.22 & 0.22 \\
\hline Num. of cells per module & 18 & 64 & 12 & 60 & 12 & 24 \\
\hline Longitudinal depth & $20.5 X_{\circ}$ & $4.4 \lambda_{\circ}$ & $4.1 \lambda_{\circ}$ & $3.6 \lambda_{\circ}$ & $4.4 \lambda_{\circ}$ & $4.4 \lambda_{\circ}$ \\
\hline Num. of readout layers & 4 & 4 & 1 & 4 & 1 & 3 \\
\hline Cells/Readout layer & $2,2,6,8$ & 16 & 12 & 15 & 12 & 8 \\
\hline Tot. radiation lengths & 20.5 & 121.8 & 32.8 & 115.5 & 37.9 & 65.1 \\
\hline Tot. absorption length $(\lambda)$ & 0.95 & 4.9 & 3.6 & 4.0 & 4.1 & 7.0 \\
\hline Sampling fraction [\%] & 11.9 & 5.7 & 1.5 & 6.7 & 1.6 & 1.6 \\
\hline$\Delta \phi$ segmentation $^{c}$ & 0.1 & 0.1 & 0.1 & 0.1 & 0.1 & 0.1 \\
\hline$\Delta \eta$ segmentation $^{d}$ & 0.1 & 0.1 & 0.1 & 0.1 & 0.1 & 0.1 \\
\hline Readout channels $^{e}$ & 14976 & 8576 & 1856 & 2944 & 768 & 1784 \\
\hline
\end{tabular}

${ }^{a}$ Uranium is depleted and $\mathrm{FH}$ (IFH and MFH) absorbers contain $1.7 \%$ Niobium alloy ${ }^{b}$ Stainless Steel

${ }^{c}$ EM3 layer has $\Delta \phi \times \Delta \eta=0.05 \times 0.05$ for $|\eta|<2.6$

${ }^{d}$ For $|\eta|>3.2, \Delta \phi=0.2 \Delta \eta \approx 0.2$

${ }^{e} \mathrm{MCH}$ and $\mathrm{OH}$ are summed together at $|\eta|=1.4$

The $\mathrm{OH}$ ring surrounds the $\mathrm{MH}$ ring at an inner radius of $162 \mathrm{~cm}$ and an outer radius of $226 \mathrm{~cm}$. Each of the $16 \mathrm{OH}$ modules are coarse, and form a parallelogram with an inner face at an angle of $27.4^{\circ}$ with respect to the $x y$ plane. An $\mathrm{OH}$ module 
consists of 25 radial cells, read out in three layers. Each cell uses $46.5 \mathrm{~mm}$ stainless steel absorber plates.

The reader is referred to Table 2.5 for a summary of the design specifications of the EC, and to Figures 2.10 and 2.11 for a layout of the calorimeter modules.

\subsubsection{Intercryostat Detectors and Massless Gaps}

In the crossover region from CC to EC (see Figure 2.10), there are several rapidity regions where a particle must travel through mostly support structures (e.g. cryostat walls, end support plates, etc.) before reaching the sampling calorimeter modules. To partially compensate for the energy loss in these support walls two different types of detectors were adopted. First, an additional layer of LAr sampling was included on the face of each $\mathrm{MH}$ and $\mathrm{OH}$ module of the $\mathrm{EC}$ and on each end of the FH modules in the CC. These massless gaps (MG) have no significant absorber material but do sample the shower energy before and after the dead material between the cryostats. The $\eta$ coverage for the MG is $0.7<|\eta|<1.2$, with a typical segmentation of $0.1 \times 0.1$ in $\eta \times \phi$ space. The second, called the Intercryostat Detector (ICD), consists of two arrays of 384 scintillation counter tiles mounted on the front surface of each EC cryostat. The tiles match the LAr calorimeter cells in size. 


\subsubsection{Calorimeter Readout}

The calorimeter signals, which consist of pulses with widths of $450 \mathrm{~ns}$, are readout in three steps. First, the signals are carried through four ports in the cryostats to charge sensitive preamplifiers mounted on top of the cryostats. Output signals from the preamplifiers are transported to the baseline subtractor (BLS) modules located in the platform underneath the detector. The BLS modules perform analog signal shaping, then split the signal into two. The first is used as input to the calorimeter Level-1 trigger (see Section 2.7.2.2) after summing the signals into $0.2 \times$ 0.2 trigger towers. The second is used for data readout: it is sampled just before the beam crossing and $2.2 \mu$ s later. The difference between the two samples is a dc voltage proportional to the collected charge. Finally, if the event is accepted by the Level-1 trigger, this difference is sent to ADCs which digitize, then zero-suppress (see Section 2.4.5) the signal before sending it on to the Level-2 trigger.

\subsubsection{Calorimeter Performance}

The performance of the calorimeter modules has been extensively studied in test beams [28, p. 210], and by using cosmic rays in situ [49]. Their response to single electrons and pions, with energies ranging between 10 and $150 \mathrm{GeV}$, is found to be linear to within $0.5 \%$. 
The energy resolution is parametrized [35, p. 271] as

$$
\frac{\delta E}{E}=\mathcal{C} \oplus \frac{\mathcal{S}}{\sqrt{E}} \oplus \frac{\mathcal{N}}{E} .
$$

The noise constant $\mathcal{N}$ is only important at low energies, and is primarily due to uranium radioactivity. The sampling term $\mathcal{S}$ is the dominant term, and is due to the sampling fluctuations. Contributions to the constant term $\mathcal{C}$ affect the resolution curve as a whole, and hence include any calibration errors. For electrons, the resolutions are measured to be

$$
\mathcal{C}=0.003 \pm 0.002, \quad \mathcal{S}=0.157 \pm 0.005 \mathrm{GeV}^{\frac{1}{2}}, \quad \mathcal{N} \approx 0.140 \mathrm{GeV}
$$

while for pions, they are

$$
\mathcal{C}=0.032 \pm 0.004, \quad \mathcal{S}=0.41 \pm 0.04 \mathrm{GeV}^{\frac{1}{2}}, \quad \mathcal{N} \approx 1.28 \mathrm{GeV}
$$

The position resolution of the EM calorimeters is found to vary between 0.8 and $1.2 \mathrm{~mm}$ as the impact position varied. This position resolution also exhibited the expected $1 / \sqrt{E}$ dependence.

Finally, the calorimeter does not turn out to be perfectly compensating: the $e / h$ ratio varies from 1.11 at $10 \mathrm{GeV}$ to 1.04 at $150 \mathrm{GeV}$.

\subsection{Muon Spectrometer}

Muons are identified by their very penetrating nature: their lifetime of $2.2 \mu \mathrm{s}$ is much larger than the scale of the detector (thus making them stable for all practical 
purposes), and their mass of $\approx 200 m_{e}$ is too large to initiate an electromagnetic shower $^{8}$. The calorimeter is made thick enough that only muons are likely to penetrate its outermost layers. These muons are detected in proportional drift tube (PDT) chambers surrounding the calorimeter. The principle of operation of these chambers is nearly identical to the VTX, CDC and FDC (see Section 2.4). In addition, their momenta are measured using a spectrometer consisting of three layers of such PDT chambers on either side of toroidal magnets (see Figure 2.2). The minimum muon momentum required to pass through the calorimeter and the iron magnets varies from $3.5 \mathrm{GeV} / \mathrm{c}$ at $\eta=0$ to $5.0 \mathrm{GeV} / \mathrm{c}$ at higher $\eta$. The momentum resolution is most easily parametrized in terms of the inverse momentum $k=1 / p$. This resolution is measured to be:

$$
\frac{\delta k}{k}=0.18 \oplus \frac{0.03}{k}
$$

The muon system is not used in this analysis, so the interested reader is referred to [28][50] for details.

\subsection{Trigger and Data Acquisition}

The Tevatron, during Run I, operated with a $3.5 \mu$ s interval between bunch crossings, which amounts to a rate of about $286 \mathrm{kHz}(\approx 1 / 3.5 \mu \mathrm{s})$. It is neither practical, nor is it interesting, to read out the entire detector at each beam crossing. Most of the physics processes of interest have a very small cross section compared to

\footnotetext{
${ }^{8}$ Muons with energies less than $\sim 500 \mathrm{GeV}$ do not readily produce an electromagnetic shower.
} 
the total (elastic and inelastic) $p \bar{p}$ cross section ${ }^{9}$. The solution is to trigger on events of interest. A trigger is defined as an electronic signal indicating the occurrence of a desired temporal and spatial correlation in the detector signals [35, p. 303]. From a practical point of view, the readout system is triggered and the event is recorded if certain conditions are met, such as the presence of a high- $E_{T}$ electron.

A schematic overview of the DØ trigger system is shown in Figure 2.12. It consists of three different levels, each with increasingly sophisticated event characterization. The Level- $\varnothing$ trigger, using a set of scintillator counters, indicates the presence of an inelastic collision: it distinguishes between beam-beam $(p \bar{p})$ and beam-gas or beam-halo collisions. It reduces the $286 \mathrm{kHz}$ rate down to about $150-200 \mathrm{kHz}$. The Level-1 trigger is hardware based: hardware elements issue decisions based on fast detector pick-offs. Most trigger decisions incur no dead-time penalty: they are made within the $3.5 \mu$ s interval between beam crossings. However, some triggers, called Level-1.5, may require additional time. The event rate out of Level-1 (and Level-1.5) is roughly $100 \mathrm{~Hz}$. Events that pass the Level-1 trigger are fully digitized, and the data is sent to a farm of 48 microprocessors, which make up the Level-2 trigger. The Level-2 trigger is software based, and relies on the reconstruction of the events with a simplified and fast version of the reconstruction program. If an event passes the Level-2 trigger, it is passed along to the host system, where it is written to magnetic tape. The rate out of Level- 2 is approximately $2 \mathrm{~Hz}$, a rate limited by the speed of the magnetic recording medium.

\footnotetext{
${ }^{9}$ At $\sqrt{\mathrm{s}}=1.8 \mathrm{TeV}$, the total $p \bar{p} \rightarrow X$ cross section is $70 \mathrm{mb}$.
} 


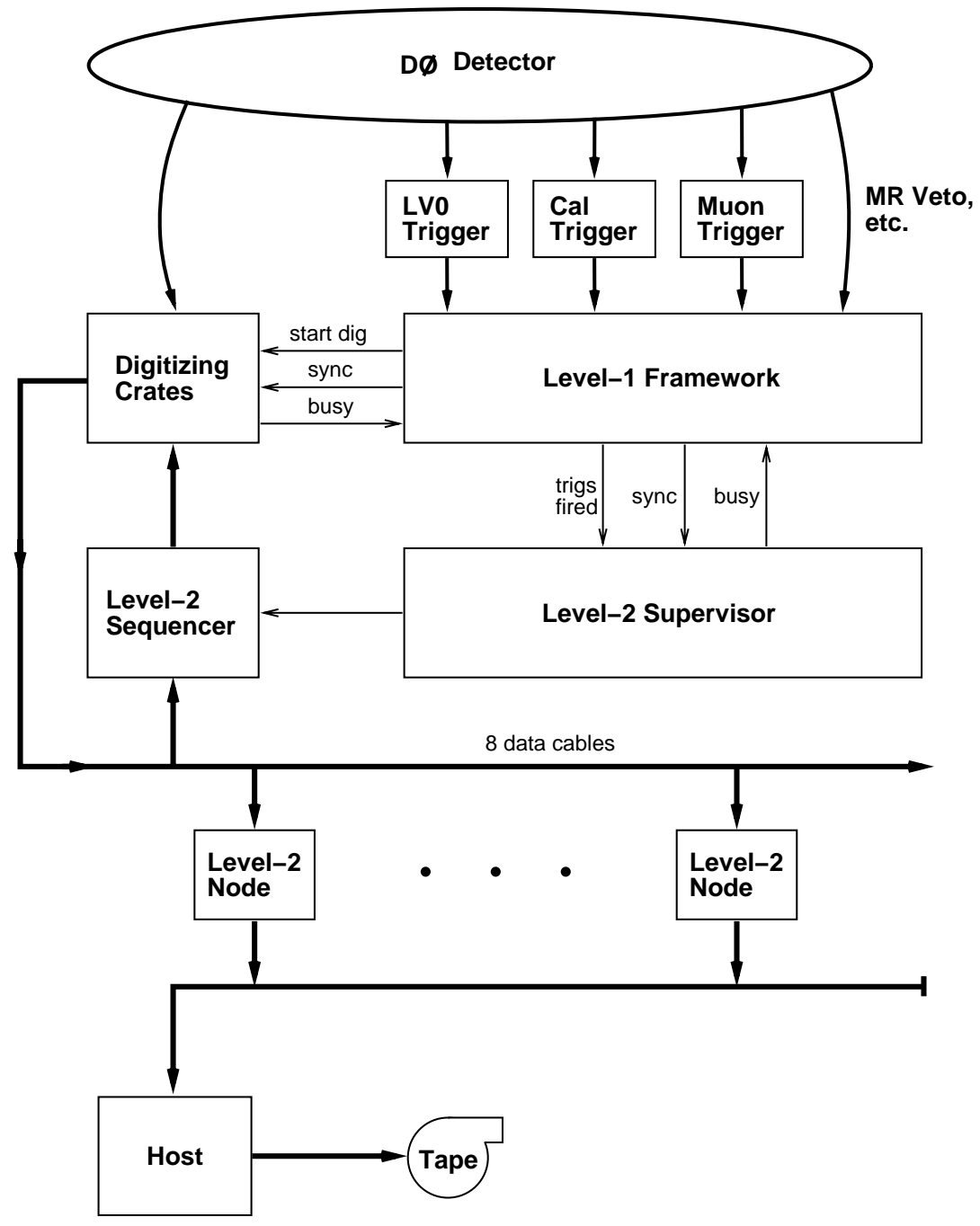

Figure 2.12: D $\varnothing$ trigger and data acquisition system.

For further discussion on triggers and data acquisition systems, the reader is referred to [51][52][53, ch. 1]

\subsubsection{Level- $\varnothing$}

The Level- $\varnothing$ system [54] performs several functions:

- Detection of inelastic $p \bar{p}$ collisions; 
- Luminosity monitoring;

- Identification of multiple interactions within one beam crossing;

- Determination of the $z$-coordinate of the interaction vertex.

The Level- $\varnothing$ system consists of two arrays of hodoscopes mounted between the FDC and EC. Each hodoscope consists of rectangular scintillation counters which provide partial coverage of the rapidity range $1.9<|\eta|<4.3$, and nearly full coverage in the range $2.3<|\eta|<3.9$. The spectator quarks in an inelastic $p \bar{p}$ collision will hadronize in the far forward region. The Level- $\varnothing$ trigger exploits this by looking for a coincidence between signals from the hodoscopes at each end of the detector. The rapidity coverage is set by the requirement that such a coincidence is greater than $99 \%$ efficient in the detection of inelastic collisions.

In addition, the arrival times of the signals from the two hodoscopes is used in determining the $z$-coordinate of the collision vertex. Due to the large spread of the vertex distribution $(\sigma=30 \mathrm{~cm})$, knowledge of $z$ position of the vertex improves all $E_{T}$ and $\mathbb{E}_{T}$ calculations at Level-1 and Level-2. A fast $z$-coordinate (fast $z$ ) determination, with a resolution of $\pm 15 \mathrm{~cm}$ is available $800 \mathrm{~ns}$ after the collision, and is used by Level-1. At $2.1 \mu$ s after the collision, a more accurate determination is made with a resolution of $\pm 3.5 \mathrm{~cm}$ (slow $z$ ), and this is provided to the Level-2 trigger. The time distribution of the counter hits is also used to determine if an event contained multiple interactions: a flag is set and made available to the trigger system. 
The rate of the Level- $\varnothing$ system is also used to determine the luminosity delivered to the $\mathrm{D} \varnothing$ detector. At low luminosities, there are very few multiple interactions, hence, the coincidence rate is almost exactly proportional to the instantaneous luminosity. At higher luminosities, the rate of multiple interactions increases, and the coincidence rate starts to saturate ${ }^{10}$. A correction is introduced in order to properly calculate the luminosity. A discussion of this issue, as well as the whole luminosity determination mechanism, will be deferred until Chapter 8 .

\subsubsection{Level-1}

The Level-1 trigger is a hardware trigger which bases its decision on fast detector data pickoffs (see Section 2.5.5), and renders most of its decisions within the $3.5 \mu \mathrm{s}$ interval between beam crossings. At its heart lies the Level-1 trigger framework [55][56], a flexible and programmable hardware processor that is responsible for combining the decisions of the individual Level-1 components, for coordinating various vetoes which can inhibit triggers, for providing a large number of scalers which allow for accounting of trigger rates and dead-times, and for managing the readout of the digitization crates before handing the event to the Level-2 trigger. The Level-1 trigger decisions are based on detector data from the calorimeter, the muon system, the Level- $\varnothing$ hodoscopes, and the accelerator timing signals. The trigger vetoes are related to any Main Ring activity (recall that the Main Ring passes through the calorimeter), as well as to any required prescales to reduce the output rate.

\footnotetext{
${ }^{10}$ Saturation occurs when a crossing with multiple interactions is recorded as a single coincidence.
} 
The Level-1 trigger framework consists of a network of 256 AND-OR terms (called latch bits). Each of these bits contains specific requirements, such as the presence of an EM trigger tower with $E_{T}>10 \mathrm{GeV}$. The 256 input AND-OR trigger terms are reduced to 32 output terms, corresponding to 32 specific Level-1 triggers. Each Level-1 trigger is a logical combination of the 256 input term, whether that term is required to be asserted, negated, or ignored. Each trigger has also a programmable prescale $^{11}$ that can be used to control the input rate to the Level-2 trigger.

When a specific Level-1 trigger is satisfied, the framework begins the digitization of the data, and informs Level-2 by sending it a mask containing all Level-1 trigger decisions. The digitization hardware resides in 86 front-end VME crates which are located in the Moving Counting House. The crates are double buffered, hence an event can be digitized while the previous one is still being transferred to Level-2. If an event requires Level-1.5 confirmation, the framework begins the digitization process, but delays the notification of Level-2. If Level-1.5 is satisfied, then Level-2 is notified; otherwise, the digitization process is aborted.

\subsubsection{Beam Vetoes}

During normal Tevatron operations, the Main Ring is continually used for antiproton production: this minimizes the down-time between stores, thus maximizing the total delivered luminosity to the detectors. Since the Main Ring passes through the DØ detector, losses in the Main Ring will show up in the detector, thus creating

\footnotetext{
${ }^{11}$ Setting the prescale to some integer value $\mathrm{N}$ causes the trigger to pass the event once in every $\mathrm{N}$ times that its trigger is satisfied.
} 
a high noise level (especially in the calorimeter). Therefore, events are not accepted during these noisy periods by enabling veto signals in the framework.

The largest Main Ring losses occur during injection and transition. Injection occurs every $2.4 \mathrm{~s}$ with transition occurring $300 \mathrm{~ms}$ later [30, p. 16]. To deal with this situation, a MRBS_LOSS veto trigger is implemented, which rejects any events within a $400 \mathrm{~ms}$ window following injection. The resultant dead-time is about $0.4 / 2.4 \approx 17 \%$ $[62]$

Another period of heavy Main Ring losses occurs when Main Ring bunches pass through the detector. A veto trigger, called MICRO_BLANK, is utilized to reject events when Main Ring bunches occur within a $1.6 \mu$ s window of the Tevatron beam crossing $^{12}$. This incurs a dead-time of about $8 \%$ [62].

\subsubsection{Level-1 Calorimeter Trigger}

The calorimeter Level-1 trigger [55][56] operates on the fast pick-off signals provided by the calorimeter's BLS cards (see Section 2.5.5), which sum cells into 1280 trigger towers of size $0.2 \times 0.2$ in $\eta \times \phi$ space out to $|\eta|=4.0$. Separate inputs are provided for the EM and the $\mathrm{FH}$ modules ${ }^{13}$, for a total of 2560 energy measurements. Each energy measurement is analog-weighted by the sine of the polar angle of the trigger tower in order to obtain the transverse energy, and then it is digitized by an 8-bit FADC. The corrected transverse energy is then computed by using the Level- $\varnothing$

\footnotetext{
${ }^{12}$ This situation occurs every $21 \mu \mathrm{s}$.

${ }^{13}$ The $\mathrm{CH}$ modules are not used at Level-1.
} 
"fast $z "$ measurement of the interaction vertex. In addition, all trigger towers are summed to produce seven global variables: the global corrected EM transverse energy $\left(E_{T}\right)$, hadronic $E_{T}$, and total $E_{T}$, and the global uncorrected EM $E_{T}$, hadronic $E_{T}$, and total $E_{T}$, as well as missing $E_{T}$. These quantities can be compared with up to 32 programmable thresholds. Each of these comparisons yields a trigger term which is input to the trigger framework. This makes it possible to specify triggers such as 'missing $E_{T}$ above $15 \mathrm{GeV}$ ', or 'total corrected $E_{T}$ above $100 \mathrm{GeV}$ '.

In addition to using the global sums, trigger terms can be formed by comparison of the individual trigger tower energies with up to four different programmable reference values. There are four sets of EM tower thresholds, each with an associated hadronic veto threshold, i.e. a trigger bit is set if the EM energy is greater than, and the hadronic energy is less than, their respective thresholds. Each of these threshold pairs constitutes an EM $E_{T}$ reference set. Furthermore, there are four thresholds used for the total energy in the tower, known as total $E_{T}$ reference set. It is worthwhile mentioning that the trigger decision can be very flexible since the 12 reference values are separately programmable for each trigger tower. For each of the eight reference sets (four EM and four total), a global count of the number of trigger towers that exceed their threshold is made. These global counts can be compared with up to 32 programmable count thresholds, the results of which are input as AND-OR terms in the Level-1 framework. This makes it possible to specify triggers such as 'one EM tower above $15 \mathrm{GeV}$ ', or 'two EM towers above $10 \mathrm{GeV}$ each'. 


\subsubsection{Level-1.5 Calorimeter Trigger}

When the $\mathrm{D} \varnothing$ detector was first commissioned, only muon L1.5 triggers were implemented. During Run Ia, it was quickly realized that $W$ and $Z$ electronic triggers would have to be prescaled in order to keep their rates at a reasonable level. The rather low $E_{T}$ thresholds used at Level-1 were motivated by the $E_{T}$ resolution of the Level-1 calorimeter trigger ${ }^{14}$ and the desire to remain fully efficient. Since the background rates for electrons grows dramatically as $E_{T}$ thresholds are lowered, it becomes advantageous to improve the resolutions and increase the background rejection. This is one of the reasons for the introduction of the calorimeter Level-1.5 trigger [57][58] during Run Ib, based on commercial digital signal processors (DSP).

The Level-1.5 calorimeter trigger is composed of two hardware pieces: the interface to Level-1 which supplies the necessary input data, and the actual trigger machinery, where the DSP boards reside inside a VME crate. The calorimeter Level-1 trigger signals are spread out across 10 physical racks. Hence, it was natural to assign essentially one local DSP per Level-1 $\operatorname{rack}^{15}$ for the local processing of candidates. Each DSP handles Level-1 candidates from all $32 \phi$ trigger towers in a patch $4 \eta$ trigger towers wide: the effective coverage is $\Delta \eta \times \Delta \phi=0.8 \times 2 \pi$. Once Level-1.5 confirmation is required, the data, consisting of the EM and total transverse energy of each trigger tower, is sent to the local DSPs at a rate of about $400 \mathrm{Mbytes} / \mathrm{s}$. The

\footnotetext{
${ }^{14}$ Both electrons and jets may split their energy across towers, thus resulting in decreased trigger efficiency.

${ }^{15}$ There is, in fact, a total of 11 such DSPs.
} 
local DSPs then proceed to rebuild the Level-1 candidate list, since it was not made available to Level-1.5.

The Level-1.5 electromagnetic nearest neighbor algorithm is then applied to all Level-1 candidates. This algorithm adds together the EM transverse energies of the seed tower and its highest $E_{T}$ neighbor in either the $\eta$ or $\phi$ direction: this sum, denoted by $(2 \times 1)_{E M}$, is required to be larger than a preset threshold. A second algorithm, the EM fraction algorithm, then requires the ratio of the $(2 \times 1)_{E M}$ transverse energy to the $(2 \times 1)_{\text {total }}$ transverse energy to pass a certain threshold. A third algorithm, the EM isolation algorithm, is also capable of deciding whether the candidate electron was isolated by applying a cut on the isolation estimator $(2 \times 1)_{E M} /(3 \times 3)_{\text {total }}$. However, the isolation algorithm was not applied in the $W$ and $Z$ triggers. This makes it possible to specify Level-1.5 triggers such as 'one EM object above $12 \mathrm{GeV}$ ', or 'two EM objects above $10 \mathrm{GeV}$ each, one of which has an EM fraction greater than $85 \%$ '.

The local DSPs then report their results to a global DSP which informs the Level-1 framework whether the Level-1 candidates were confirmed or not, which ultimately leads to a decision on whether the event should be sent to Level-2, or should be discarded.

\subsubsection{Level-2}

The Level-2 system [59][60] functions as the D $\varnothing$ data acquisition system and the Level-2 software trigger. It is responsible for collecting the digitized data from Level-1, 
formatting it into a dynamic data structure called ZEBRA ${ }^{16}$, applying software event filtering, and sending events of interest to the online host system. The goal of the Level-2 system is the reduction of the $100 \mathrm{~Hz}$ input rate to $2 \mathrm{~Hz}$, while retaining events of interest.

The Level-2 system is composed of 48 parallel microprocessors that are connected to the Level-1 framework by a set of eight 32-bit wide high speed data cables, each capable of a transfer rate of 40 Mbytes/s. These data cables form a loop which originates and terminates at a sequencer card. The VME crates, which house the digitizing hardware, contain a VME buffer/driver (VBD) card each. These VBD cards are connected to one of the eight data cables. When a crate has finished digitizing, the VBD copies the data into an internal buffer and waits until the sequencer card instructs it to read it out over the data cables.

The microprocessors are DEC Vax-stations (nodes) running the VAXELN ${ }^{17}$ real time operating system. Each Level-2 node is connected to its own VME crate and a VBD card. Each VME crate contains four dual multi-port memory (MPM) boards which are in turn connected to the eight data cables. The MPM memory, which appears as contiguous I/O space memory to the Level-2 node, holds the data while event filtering is in process. Each Level-2 VBD card buffers the data for transfer to the host system.

\footnotetext{
${ }^{16}$ ZEBRA [63] is an extension of the FORTRAN programming language which allows for dynamic memory allocation.

${ }^{17}$ VAXELN is designed to guarantee a precise response to every required system task; it performs no swapping or page faulting.
} 
The actual control of the eight data cables, as well as the transfer from the digitizing crates to a Level-2 node, is performed by the Level-2 supervisor. When the Level-1 framework informs the supervisor that an event is ready, the supervisor polls the Level-2 nodes to find one available, then enables its MPMs to receive the data from the cables. The supervisor then informs the sequencers to begin the transfer of the data in the VBD buffers to the MPMs. Once the target Level-2 node has received the complete data for an event, the event is converted into the ZEBRA format. The node is now ready for event filtering.

\subsubsection{Level-2 Filter}

Software filtering [61] of events on each of the Level-2 nodes is accomplished by a series of filter tools. Each tool has a specific function related to identification of a type of particle or event characteristic. Jets, muons, calorimeter EM clusters, track association with calorimeter clusters, scalar $E_{T}\left(\Sigma E_{T}\right)$, and missing $E_{T}$, have their own filtering tools. For example, an electron filter tool may depend on a minimum number of calorimeter EM clusters, minimum $E_{T}$ for each cluster, and track association with the clusters. The tools are associated in particular combinations and ordered into scripts. Each of the 32 Level-1 trigger bits is associated with one or more scripts. For example, a single electron trigger from Level-1 can have several Level-2 scripts depending upon the $E_{T}$ threshold or other features in the event (e.g. energy isolation or the presence of missing $E_{T}$ ). There are a maximum of 128 Level-2 scripts. 
For each Level-1 bit that is passed, a call is made to its Level-2 associated scripts. If any of the Level-2 scripts are passed, the event is sent to the online cluster to be logged and recorded on permanent storage media.

\subsection{Online Cluster}

The D $\varnothing$ online cluster [64, sec. 9] serves as the interface to the detector systems. It is composed of three DEC VAX computers supplemented by additional satellite nodes and X-window terminals. The online cluster is responsible for high level control of the data-taking system, downloading all set table parameters (i.e. trigger configuration), specifying hardware monitoring activities, and the recording and displaying of data collected by the detector ( $p \bar{p}$ interactions, calibrations, monitoring information, alarms). The data-taking system is designed to support flexibly defined partitions of the detector: it allows multiple users to collect individually tailored data streams. Upon receiving an event from Level-2, the host system logs it to a staging disk, dispatches a sample for online monitoring purposes, and spools the events from the staging disk to $8 \mathrm{~mm}$ Exabyte tapes.

\subsection{Offline Data Processing}

The raw data recorded on $8 \mathrm{~mm}$ tapes are reconstructed on a farm of SGI and IBM machines. The number of nodes in the farm was dependent on demand and 
consisted of as many as 96 nodes during Run I. The output of the reconstruction program consists of two sets of files: STA and DST. The STA files, ranging from 600-1000 kbytes/event, contain the raw data of the event, along with the results of the reconstruction. The DST files contain a summary of the event data, along with the reconstruction results for high-level objects, such as electrons, photons, muons, and jets. The DST files are about 15 kbytes/event. However, the enormous size of the Run I data sample necessitated the creation of a third file type, the $\mu \mathrm{DST}$. $\mu$ DSTs contained the minimum amount of information needed to carry out the physics analyses. All the $\mu \mathrm{DST}$ s were placed on disks, and made available to the users via the Dø file server. 


\section{Chapter 3}

\section{Event Reconstruction and Particle}

\section{Identification}

The information recorded by the $\mathrm{D} \emptyset$ detector is in the form of digital signals: pulse heights, widths and times, which need to "interpreted" as physics objects. This complicated and difficult task is performed by the standard reconstruction software package (DØRECO). DØRECO starts by processing the raw data into high-level objects, such as energy clusters in the calorimeters or tracks in the tracking and muon systems. These objects are in turn combined to form the physical particles that originated in the $\bar{p} p$ collisions: electrons, photons, jets, muons, and neutrinos $\left(\mathbb{E}_{T}\right)$. These particles and their measured kinematic properties form the basis of all analyses; therefore, it is essential to fully understand the reconstruction process. A full account of the reconstruction process is outside the scope of this dissertation, so a brief description will be presented, with emphasis given to the relevant pieces which affect this analysis. The interested reader is referred to [65] for further details. 


\subsection{Track Reconstruction}

Track reconstruction in the Central Drift Chamber is categorized as follows:

- Pulse and Hit Finding;

- Segment Finding;

- Segment Matching and Global Track Fit.

First, the raw FADC data, for any given sector and layer, are unpacked. The data contains the digitized charge versus time bin with the associated wire address. The identification of individual pulses is achieved by looking for leading and trailing edges. Each pulse is integrated to compute the total deposited charge (this is later used in computing $d E / d x)$. After performing channel-to-channel variation corrections, the time of arrival of a pulse is used to determine the position of the pulse: the time required to drift to the sense wire gives the distance of the hit from the sense wire, while the arrival time of the pulse from the delay line gives its location along the sense wire. Since the drift volume is divided by the sense wire into two symmetric halves, individual drift times cannot allow one to distinguish from which side of the wire the electrons drifted. This is commonly referred to as left/right ambiguity: one hit corresponds to the true track, while the other is its mirror image. In practice, this ambiguity is resolved by stagerring the sense wires, thus breaking the symmetry between the two halves. The set of hits originating from the true track lends itself 
to a better straight line fit than the set from mirror images. Once all hits are found, track segment finding begins.

The segment finding process connects groups of hits within a single layer. This process is performed using a road method in the $r-\phi$ plane (the $z$ information is added to the segment afterwards). The road is defined by a pair of hits which span the sector: one hit is from the inner most wire, and one from the outer most. Due to the absence of a central magnetic field, all roads are straight. In addition, they are constrained to be nearly radial since the tracks originate from the interaction vertex and are not subjected to any significant multiple scatter. The width of the road is chosen such as full efficiency is retained while minimizing the number of fake track segments: the road width is roughly fives times the single hit resolution. All hits on intermediate wires within the road are then considered. When a sufficient predetermined number of hits is found, an overall straight line fit is performed: a segment is formed if the resultant $\chi^{2}$ is small enough $\left(\chi^{2} /\right.$ degree of freedom $\left.<10\right)$. Once all track segments are found, segment linking into tracks is straightforward. In order to be linked, two segments must lie along the same line: they must point in the same direction in space, and their spatial mismatch in the mid-plane dividing the two layers must be small. A final straight line fit is performed using all the hits from the linked segments: if the fit is good enough, and a minimum of three (out of four) layers are found, then a track is formed.

The track reconstruction outlined above is in fact applied for all three central drift chambers (VTX, CDC, and FDC). For each track, directional information is 
specified by five parameters: the coordinates of a reference point $\left(x_{0}, y_{0}, z_{0}\right)$, called the track centroid or track center-of-gravity, along with the polar angle $\theta$, and the azimuthal angle $\phi$. The track centroid is then corrected for any biases in the delay lines. In particular, the $z$-coordinate of CDC tracks has been calibrated using cosmic ray [66] and collider muons [67]. It should also be mentioned that hit finding and track reconstruction in the forward direction is only performed within wide roads defined by calorimeter clusters and the event vertex. All hits and tracks are reconstructed in the central region. For further details regarding central detector hit finding and tracking, please consult [43][44][45][46].

\subsection{Vertex Finding}

Once all the tracks in the event are found, the position of the interaction vertex (or vertices) is performed. The method employed by DØRECO to find the $z$ coordinate of the vertex utilizes a histogram of track z-intercepts (see Figure 3.1). Each CDC track is projected onto the beam-line (at $x \approx y \approx 0$ ), and the interaction vertices are identified as peaks in the resultant distribution of $z$-intercepts. The peak with the largest number of associated tracks is considered to be the "primary" or "hard scatter" vertex: all high- $p_{T}$ objects are assumed to originate from this vertex. The remaining "secondary" vertices ${ }^{1}$ are considered to arise from minimum

\footnotetext{
${ }^{1}$ In this dissertation, "primary" and "secondary" refer to the multiplicities associated with the vertices; this has nothing to do with the decay of any long-lived particles.
} 

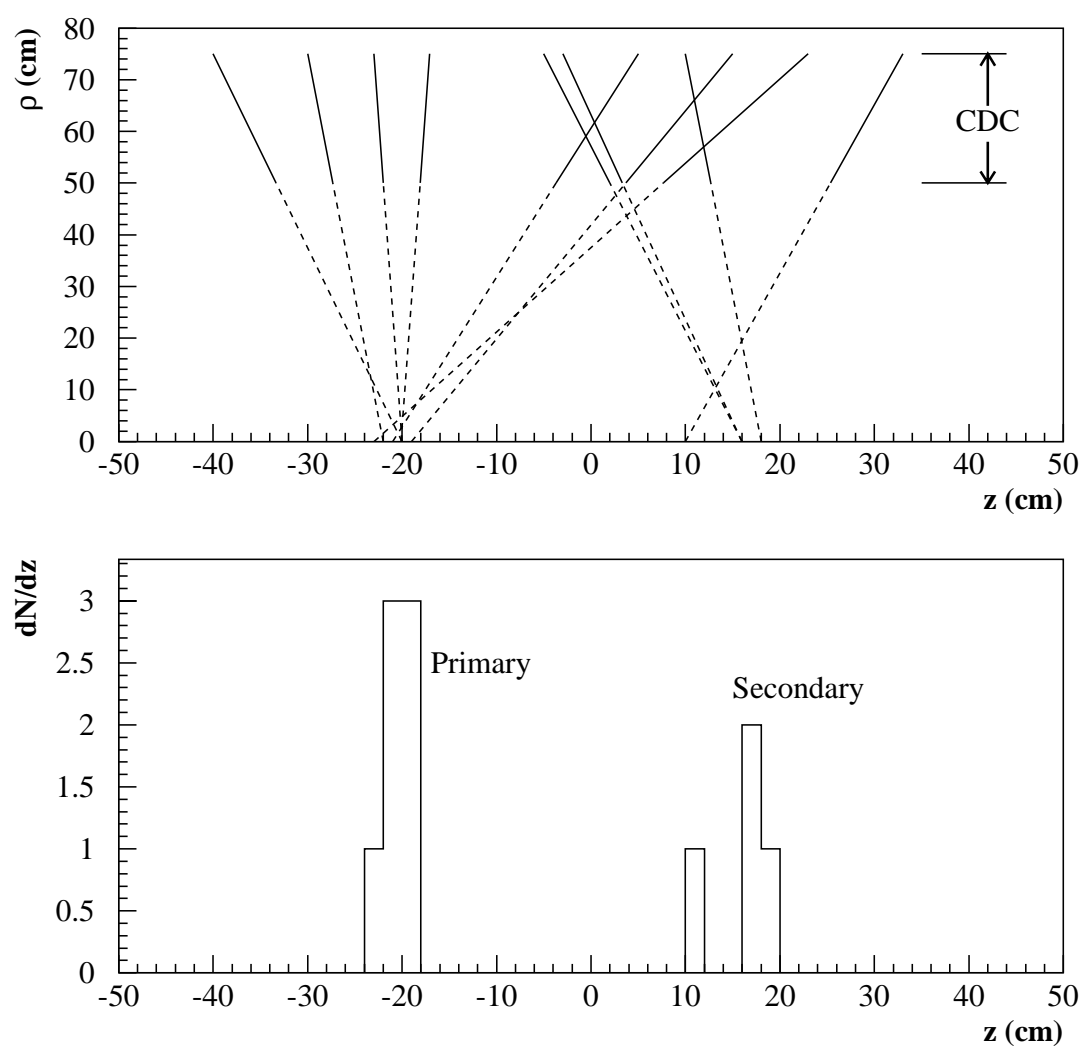

Figure 3.1: Vertex $z$ coordinate determination by the histogram method. Top: projections of CDC tracks to the beam-line (view is integrated over all azimuthal angles $\phi$ ). Bottom: resultant distribution of $z$-intercepts from which vertices are determined [68, p. 72].

bias interactions(s) in the event. This method typically achieves vertex $z$ resolutions of 1-2 $\mathrm{cm}$, with multiple vertices being identified if they are separated by at least $7 \mathrm{~cm}$. Unfortunately, this histogram vertexing method becomes unreliable in a high-luminosity environment where the number of interactions per crossing increases, or the interesting high- $p_{T}$ interaction happens to have a lower total charged track multiplicity than the other minimum bias interactions in the event. For the typical luminosity of Run I $\left(\sim 7 \cdot 10^{30} \mathrm{~cm}^{-2} \mathrm{~s}^{-1}\right)$, there were, on average, 1.2 interactions 
per event (i.e. $70 \%$ of all events contained more than one interaction). Thus a correction is introduced which is based on the angular information associated with the high- $p_{T}$ objects in the event (particularly electrons). Discussion of this correction will be postponed until Section 3.8.1, after the reconstruction of electrons has been described.

The transverse $(x, y)$ position of the vertex is not measured on an event-byevent basis. The beam spot in the transverse direction was very well known because the cross sectional area of the beam, roughly $50 \mu \mathrm{m}$, was minimized in this plane in order to achieve maximum luminosity. The beam spot was monitored on a store-bystore basis using the VTX chamber. It is interesting to note that the beam was not perfectly centered in the detector, with an offset of $3-4 \mathrm{~mm}$. This shift was noticeable in the detector data, but had very little effect on the physics analysis.

\subsection{Calorimeter Hit Finding}

The deposited energy recorded as digitized counts in the calorimeter cells has to be converted back to a physical energy in units of $\mathrm{GeV}$. The conversion factors were determined from the responses of the modules to the known energies of the test beam particles [49]. All appropriate corrections are applied, including run dependent gain corrections (such as cell-to-cell variations in the electronics gain and pedestal values), absorber thickness corrections, liquid argon purity and temperature corrections, etc. Each cell that passed zero suppression has its address bits converted to the physical 
indices in the calorimeter (eta and phi indices identify the calorimeter tower, and a layer index identifies the depth of the calorimeter cell). Hence, the energy conversion can be expressed as follows [65, p. 91]:

$$
E_{\text {cell }}(e, p, l)=A(d) \times W(e, l) \times C(e, p, l) \times G(e, p, l) \times A D C(e, p, l)
$$

where $E_{\text {cell }}$ is the cell energy in $\mathrm{GeV}$, and $(e, p, l)$ correspond to:

- $e=$ calorimeter $\eta$ index: $-37 \leq e \leq 37$;

- $p=$ calorimeter $\phi$ index: $1 \leq p \leq 64$;

- $l=$ calorimeter depth (layer) index: $1 \leq l \leq 17$.

$A$ is an overall calibration constant which depends on the module type: central calorimeter (CC), end calorimeter (EC), inter-cryostat detector (ICD), CC massless gap (CCMG), or EC massless gap (ECMG); it contains the conversion from adc counts to $\mathrm{GeV}$, as well as any needed high-voltage correction. $W$ is the sampling fraction weight, determined from test beam data, which provides the best energy resolution. $C$ contains all non run dependent corrections such as absorber thickness in the $\mathrm{CC}$ and $\mathrm{EC}$, or the ICD minimum ionizing signal corrections. $G$ contains the run dependent electronic gain corrections, such as response corrections due to capacitance or timing (derived from calibration runs), or shorted or missing channels. Finally, $A D C$ is the digitized cell energy in raw adc counts.

$\mathrm{D} \varnothing$ defines for each calorimeter cell $(e, p, l)$ the directed energy vector

$$
\vec{E}_{\text {cell }}(e, p, l)=\hat{n} E_{\text {cell }}(e, p, l)
$$


where $\hat{n}$ is the unit vector pointing from the interaction vertex to the center of the cell $(e, p, l)$ and $E_{c e l l}(e, p, l)$ is the magnitude of the energy deposited in that cell as calculated in Equation 3.1. The cell energy can then be decomposed into its vectorial components:

$$
E_{x}=E \sin \theta \cos \phi \quad E_{y}=E \sin \theta \sin \phi \quad E_{z}=E \cos \theta
$$

and

$$
E_{T}=\sqrt{E_{x}^{2}+E_{y}^{2}}=E \sin \theta
$$

The final step in calorimeter hit-finding consists in summing the energies for all the cells in each $\eta-\phi$ tower. This is done by summing over the layer index, $l$, for each tower. For towers near the cryostat boundaries, this sum includes any contributions from the massless gaps and the ICD. The sum is performed separately for the total energy and the electromagnetic (EM) energy. The EM energy includes the four layers of the electromagnetic calorimeter as well as the first layer of the fine hadronic $(\mathrm{FH})$ calorimeter $^{2}$. From the cells that constitute a calorimeter tower $(e, p)$, the energy is defined to be:

$$
E_{\text {tower }}^{E M}(e, p)=\sum_{l=1}^{8} E_{\text {cell }}(e, p, l)
$$

and

$$
E_{\text {tower }}^{T O T}(e, p)=\sum_{l=1}^{17} E_{c e l l}(e, p, l)
$$

\footnotetext{
${ }^{2}$ For EM towers, the layer index $l$ runs from 1 through 8. Out of a total of 17 possible layers, EM1 and EM2 have layer indices $l=1$ and $l=2$, the finer segmentation in EM3 takes up $l=3-6$, EM4 has $l=7$, and FH1 has $l=8$ (see Figure 2.11).
} 
For each tower, the vectorial components of its EM and total energy are also computed from the vectorial components of the cell energies:

$$
E_{x}^{\text {tower }}=\sum_{\text {layers }} E_{x}^{\text {cell }} \quad E_{y}^{\text {tower }}=\sum_{\text {layers }} E_{y}^{\text {cell }} \quad E_{z}^{\text {tower }}=\sum_{\text {layers }} E_{z}^{\text {cell }}
$$

and

$$
E_{T}^{\text {tower }}=\sqrt{\left(E_{x}^{\text {tower }}\right)^{2}+\left(E_{y}^{\text {tower }}\right)^{2}}
$$

Related kinematic quantities are then computed:

$$
\begin{aligned}
& \phi_{\text {tower }}=\arctan \left(\frac{E_{y}^{\text {tower }}}{E_{x}^{\text {tower }}}\right) \\
& \theta_{\text {tower }}=\arccos \left(\frac{E_{z}^{\text {tower }}}{E^{\text {tower }}}\right)
\end{aligned}
$$

and

$$
\eta_{\text {tower }}=-\ln \left[\tan \left(\frac{\theta_{\text {tower }}}{2}\right)\right]
$$

These tower energies form the building blocks, or seeds, of the jet and electron cluster finding algorithms.

\subsection{Missing Energy}

Neutrinos (and possibly other weakly interacting neutral particles) are not directly detected in conventional high-energy collider detectors. Their presence is inferred from an overall momentum imbalance in the event. When momentum conservation between initial and final state particles is applied, their kinematic properties are derived from the vector sum of the particles that are detected. Since energy flow 
near the beam-line is undetected (mainly due to the spectator quarks), this method can only be employed in the plane transverse to the beam. As the initial transverse momentum of the quark-antiquark system is small $(\sim 300 \mathrm{MeV})$, one expects the final transverse momentum to be small as well. When a high- $p_{T}$ neutrino is produced, the negative vector resultant of the detected particles will match the neutrino's momentum vector. This quantity, referred to as missing $E_{T}$ and denoted as $\mathbb{E}_{T}$, is used to indicate their presence.

The calculation of $\mathbb{E}_{T}$ is based upon energy deposits at the calorimeter cell level. A missing transverse energy vector, $\vec{E}_{T}$, is defined so that it cancels exactly the total transverse energy vector in the calorimeter:

$$
\mathbb{E}_{x}=-\sum_{e, p, l} E_{x}(e, p, l) \quad \mathbb{E}_{y}=-\sum_{e, p, l} E_{y}(e, p, l)
$$

and

$$
\vec{E}_{T}=\left(\begin{array}{c}
E_{x} \\
E_{y}
\end{array}\right) \text {. }
$$

The missing transverse energy, $\mathbb{E}_{T}$, is just the magnitude of this vector:

$$
\mathscr{E}_{T}=\left|\vec{E}_{T}\right|=\sqrt{\mathscr{E}_{x}^{2}+\mathbb{E}_{y}^{2}}
$$

while the azimuthal direction of $\vec{H}_{T}$ is:

$$
\phi_{E_{T}}=\arctan \left(\frac{\not E_{y}}{\not E_{x}}\right) \text {. }
$$


DØRECO computes three versions of the transverse missing energy. The first is based on the energy imbalance of the calorimeter cells only. The second version includes corrections from the massless gaps and the ICD. The third version incorporates the momenta of any reconstructed muons into the momentum balance.

\subsection{Jet Reconstruction}

When a quark or gluon (parton) emerges from the hard scatter, it cannot remain free: color confinement implies that it will appear as a jet. The process of turning a colored parton into a jet is called hadronization, since the quark produces a large number of colorless hadrons that appear in the detector as a collimated spray of hadronic particles. The process of jet identification involves finding these jets within the calorimeter, and measuring their kinematic features in order to relate them to the original parton. Although several algorithms can be used for jet reconstruction, the most common is the "fixed cone" algorithm. Specifically, the algorithm uses a fixed cone radius $\mathcal{R}$ in $\eta \times \phi$ space, where $\mathcal{R}=\sqrt{\Delta \eta^{2}+\Delta \phi^{2}}$ describes the maximum transverse radius of the jet. Four values of $\mathcal{R}$ were used, namely $\mathcal{R}=0.3,0.5,0.7$ and 1.0 , but most analyses used $\mathcal{R}=0.5$. The reconstruction algorithm is dependent on the total transverse energy in the calorimeter towers, $E_{T}^{\text {tower }}$ as defined in Equation 3.8, and is implemented as a three step process:

1. Preclustering of towers;

2. Cone clustering of preclusters; 
3. Splitting/Merging of cone clusters.

The first step in preclustering is the ordering of calorimeter seed towers in decreasing $E_{T}$. Seed towers are calorimeter towers with a minimum transverse energy of $1 \mathrm{GeV}$. The highest $E_{T}$ calorimeter seed tower is used as the starting point of the precluster. Adjacent seed towers are added to the precluster if they are within \pm 0.3 units in $\eta$ and \pm 0.3 units in $\phi$. Once seed towers are included in a precluster, they are then removed from the seed tower list. The remaining seed tower with the highest $E_{T}$ in the list is then used as the starting point of the next precluster. Preclustering continues until all of the seed towers have been exhausted. Once all seed towers are assigned to a precluster, the preclusters are ordered in decreasing precluster $E_{T}$, where the precluster $E_{T}$ is the scalar sum of all towers' $E_{T}$ in the precluster.

Cone clustering begins with the $E_{T}$ ordered list of preclusters. For each precluster, the $E_{T}$ weighted $(\eta, \phi)$ centroid is calculated and identified as the cone axis. All towers within a radius $\mathcal{R}$ of that axis are assigned to the cone cluster. The cone axis is recalculated using these towers, and the process is iterated until the cone axis becomes stable ${ }^{3}$. If the resultant cone has $E_{T}>8 \mathrm{GeV}$, the cone cluster is kept and is identified as a jet, with the jet axis defined by the stable cone cluster axis. Splitting/merging is then attempted on that jet.

Jets are not allowed to share energy: each calorimeter cell must belong to a maximum of one jet. The first jet, by definition, can share no energy with a previously

\footnotetext{
${ }^{3}$ The cone axis is considered stable if it moves less than 0.01 in $\eta \times \phi$ space between iterations, or if the maximum number of 50 iterations has been reached. The latter is introduced to prevent the rare case of a bi-stable solution from using an unreasonable amount of computer processing time.
} 
found jet. When the second and subsequent jets are found, a check is made to determine whether the new jet shared any towers with previously found jets. If one or more towers are shared, the jet axes are compared: if the jets are separated by less than 0.01 in $\eta \times \phi$ space, then the two jets are considered to be the same ${ }^{4}$, and the most recently constructed jet is dropped. In the case that the new jet is not identical to any of the previous ones, then the fraction of the $E_{T}$ sum of the shared towers to the lowest $E_{T}$ jet is calculated. If this fraction is greater than 0.5 , the two jets are merged into a single jet with all towers assigned to the combined jet. Otherwise, both jets are preserved, and each shared cell assigned to the jet whose axis is nearest to it. In either case, the jet axis is recalculated one last time, and the relevant kinematic variables are calculated for all appropriate cone clusters. On average, $5 \%$ of the jets are merged, and $30 \%$ are split. Once splitting/merging is completed, the cone clustering process is repeated until all preclusters have been exhausted.

Once the clustering process is complete, the kinematic properties of the reconstructed jets are determined by summing over the towers contained in that jet. The energy components of the jet are defined to be:

$$
E_{i}^{j e t}=\sum_{k=1}^{\text {ntowers }} E_{i}^{k} \quad(i=x, y, z, \text { total })
$$

and the $E_{T}$ of the jet:

$$
E_{T}^{j e t}=\sum_{k=1}^{n \text { towers }} E_{T}^{k}=\sum_{k=1}^{n \text { towers }} \sqrt{\left(E_{x}^{k}\right)^{2}+\left(E_{y}^{k}\right)^{2}} .
$$

\footnotetext{
${ }^{4}$ It is possible to find the same jet from more than one preluster.
} 
The jet angles are then computed as:

$$
\begin{aligned}
\phi^{j e t} & =\arctan \left(\frac{E_{y}^{j e t}}{E_{x}^{j e t}}\right) \\
\theta^{j e t} & =\arccos \left(\frac{E_{z}^{j e t}}{E_{\text {total }}^{j e t}}\right)
\end{aligned}
$$

and

$$
\eta^{j e t}=-\ln \left[\tan \left(\frac{\theta^{j e t}}{2}\right)\right] .
$$

It is worthwhile to note that the transverse and total energy of the jet is computed from the sum of the individual transverse or total tower energies, and not the magnitude of the vector components.

\subsection{Electron Reconstruction}

The showers from electrons and photons are very similar (see Section 2.5): narrow concentrated clusters of energy deposited mainly in the electromagnetic layers of the calorimeter. The only distinguishing feature is the association of the electron cluster with a track in the central tracking chambers. Hence, the reconstruction of these objects proceeds along the same line.

In contrast to the fixed cone algorithm used in jet reconstruction (see Section 3.5), the reconstruction of electrons and photons uses a "nearest neighbor" (NN) algorithm [69][70] based on the energy (not $E_{T}$ ) of electromagnetic towers ${ }^{5}$ as defined in Equation 3.5. The towers are grouped together by connecting each EM tower with

\footnotetext{
${ }^{5}$ Recall that an electromagnetic tower consists of the four EM and the first hadronic (FH1) layers of the calorimeter.
} 
other EM towers in its local neighborhood. The neighborhood is defined as an array of $3 \times 3$ towers in $\eta \times \phi$ space centered on the tower. Tower connections are made if the neighboring tower has an energy above $50 \mathrm{MeV}$. These connections define clusters of mutually connected towers in the calorimeter. Unlike the fixed cone algorithm, there is no sharing of towers between clusters: a tower is included in at most one cluster.

For each calorimeter cluster found, its kinematic properties are computed from the cell energies ${ }^{6}$. The cluster energy and transverse energy are:

$$
E_{i}^{\text {clus }}=\sum_{k=1}^{\text {ncells }} E_{i}^{k} \quad(i=x, y, z, \text { total })
$$

and

$$
E_{T}^{\text {clus }}=\sqrt{\left(E_{x}^{\text {clus }}\right)^{2}+\left(E_{y}^{\text {clus }}\right)^{2}}=\sqrt{\left(\sum_{k=1}^{n c e l l s} E_{x}^{k}\right)^{2}+\left(\sum_{k=1}^{n c e l l s} E_{y}^{k}\right)^{2}}
$$

while the angles are:

$$
\begin{aligned}
& \phi^{\text {clus }}=\arctan \left(\frac{E_{y}^{\text {clus }}}{E_{x}^{\text {clus }}}\right) \\
& \theta^{\text {clus }}=\arccos \left(\frac{E_{z}^{\text {clus }}}{E_{\text {total }}^{\text {clus }}}\right)
\end{aligned}
$$

and

$$
\eta^{\text {clus }}=-\ln \left[\tan \left(\frac{\theta^{\text {clus }}}{2}\right)\right]
$$

In addition, the energy deposited in the first hadronic layer, $E_{\text {had }}$, is computed, as well as the transverse profile, $E_{\text {trans }}$, which is defined as the energy outside the hottest tower (in $\eta$ direction) of the cluster.

\footnotetext{
${ }^{6}$ Recall that for jets, the tower energies were used.
} 
At this point, all possible clusters have been identified. Those which pass the following criteria are then considered as electron or photon candidates [65, pp. 114115]:

- The total cluster energy $E_{\text {total }}>1.5 \mathrm{GeV}$;

- The total transverse cluster energy $E_{T}>1.5 \mathrm{GeV}$;

- The electromagnetic energy fraction is greater then $90 \%$, or $E_{\text {had }} / E_{\text {total }}<0.1$;

- The hottest tower (i.e. the one containing the most energy) of the cluster must account for at least $40 \%$ of the cluster energy, or $E_{\text {trans }} / E_{\text {total }}<0.6$.

For electron or photon candidates, the cluster centroid, $\vec{x}_{c l u s}$, is calculated by forming a weighted mean of the coordinates $\vec{x}_{i}$ of the cluster cells in the finely segmented EM3 layer [65, p. 119]:

$$
\vec{x}_{c l u s}=\frac{\sum_{i} w_{i} \vec{x}_{i}}{\sum_{i} w_{i}} .
$$

The weights $w_{i}$ are based on the logarithm of the cell energy $E_{i}$ :

$$
w_{i}=\max \left(0, w_{0}+\ln \left(\frac{E_{i}}{E_{\text {clus }}}\right)\right)
$$

where $w_{0}$ is a parameter chosen to optimize the position resolution. These weights are found to be $\eta$ and $\phi$ dependent, and were tuned using test beam data. This logarithmic weighting scheme is motivated by the exponential lateral profile of the shower [71][72]. In addition, small corrections to the centroid position are made which account for the entry angle of the electron into the calorimeter [73]. 
At this juncture, a distinction is made between electrons and photons. Tracking roads are defined between the calorimeter clusters and the primary interaction vertex position. Azimuthally, the road covers \pm 0.1 radians around the cluster position. The road limits in $\theta$ are determined as follows [65, p. 115]:

$$
\tan \theta_{ \pm}=\min \left(\frac{\rho_{\text {clus }}}{\left(z_{\text {clus }}-z_{v t x} \pm \delta z\right)}, 0.1\right)
$$

where $\vec{x}_{\text {clus }}=\left(x_{\text {clus }}, y_{\text {clus }}, z_{\text {clus }}\right)$ are the coordinates of the cluster centroid, $\rho_{\text {clus }}=$

$\sqrt{x_{\text {clus }}^{2}+y_{\text {clus }}^{2}}, z_{v t x}$ is the $z$-coordinate of the primary vertex, and $\delta z$ its corresponding error. A search for central detector tracks is performed in this road. If one or more tracks are found, then the candidate cluster is classified as an electron; otherwise, it is classified as a photon. Clearly, this distinction becomes problematic if the vertex position is incorrectly identified and the tracking roads are miscalculated. A more robust method which does not rely on the vertex position has been developed to deal with this shortcoming, and will be described in Section 3.8.1.

\subsection{Electron Identification}

The emphasis of the algorithms used in DØRECO is towards maximum efficiency in the reconstruction of electrons and photons. This implies that a fair amount of background is present at this point, and that the task of further separating it from the real signal is left to the individual analyses. Standard techniques have been developed in the identification of electrons, which introduce additional criteria that reduce the background considerably while retaining most genuine electrons for the 
analysis. Two of the criteria rely on calorimeter information and exploit the difference between an electromagnetic and a hadronic shower: the electromagnetic energy

fraction $\left(f_{e m}\right)$ and the H-matrix chi-squared $\left(\chi_{h m}^{2}\right)$, where the latter is derived from an analysis of the shower shape. The third criterion, shower isolation fraction $\left(f_{i s o}\right)$, also relies on calorimetric information. However, this criterion is not based on the shower itself: it is a topological cut which is consistent with the decay of electrons from $W$ and $Z$ gauge bosons. Finally, the fourth criterion is based on calorimetric and tracking information: track match significance $\left(S_{t r k}\right)$ quantifies the quality of the track matching performed for electrons.

\subsubsection{Electromagnetic Energy Fraction}

Electrons and photons have, by definition, a large electromagnetic fraction (seeSection 3.6): 90\% of the cluster energy must be deposited in the EM layers of the calorimeter. For electrons originating from decays of $W$ and $Z$ bosons, that requirement is quite loose. Figure 3.2 shows the distribution of $f_{e m}$ for electrons from $Z \rightarrow e e$ decays, and electrons from multi-jet events. The former is dominated by signal, while the latter is dominated by background. Added background rejection is obtained by raising the cut to $f_{e m}>0.95$. 

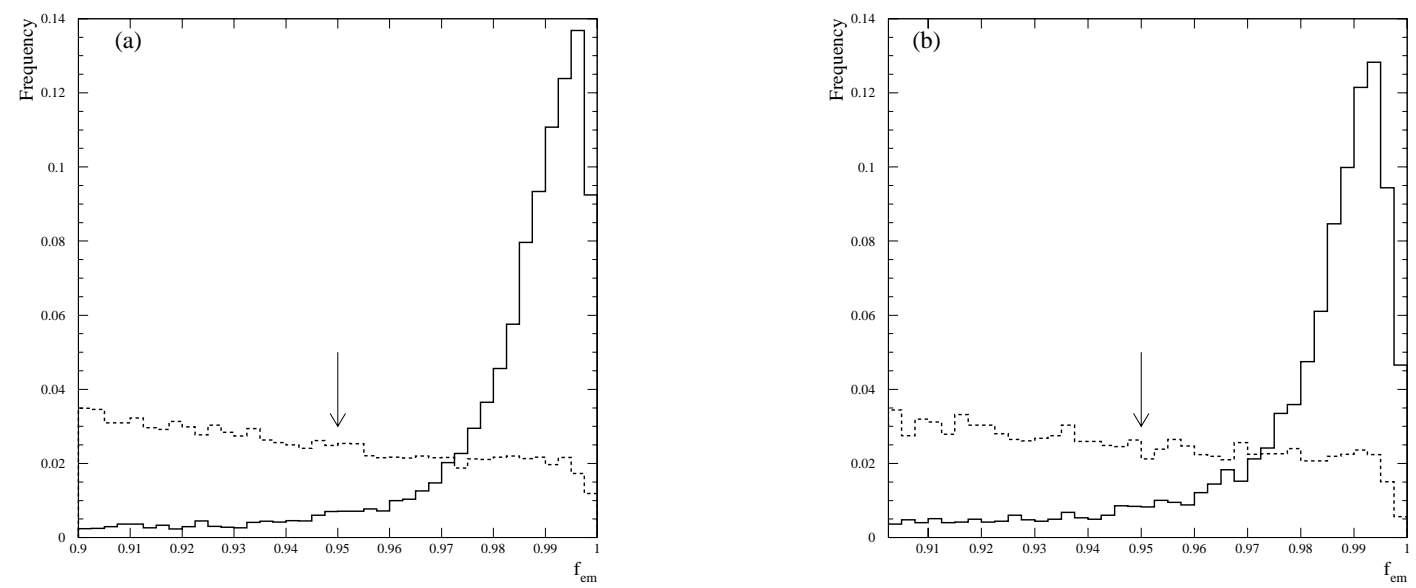

Figure 3.2: EM fraction $f_{\text {em }}$ distribution for electrons from $Z \rightarrow e e$ candidates (solid) and electrons in multi-jet triggered data (dashed), for (a) central electrons and (b) forward electrons.

\subsubsection{Shower Shape Analysis}

The shower shape of an electromagnetic object (electron or photon) can be characterized by its longitudinal and transverse profile: it is dependent on the fraction of cluster energy deposited in each cell of the calorimeter. The fractions, besides being dependent on the incident electron energy and impact position, are also correlated: a shower which fluctuates and deposits a large fraction of its energy in the first layer will then deposit a smaller fraction in the subsequent layers and vice versa.

To fully account for all possible correlations, a covariance matrix $M$ of 41 observables is built which characterizes the "electron-ness" of the shower [74][75][76]. The observables are the fractional energies in layers EM1, EM2 and EM4 of the calorimeter, and the fractional energy in each cell of a $6 \times 6$ array of EM3 cells in $\eta \times \phi$ space centered on the most energetic tower in the cluster. In addition, the logarithm of the cluster energy is included as an observable to account for the dependence 
of the fractional energy deposits on the cluster energy. Finally, the z-coordinate of the interaction vertex is included, to account for the dependence of the shower shape on the angle of incidence into the calorimeter. Since the calorimeter geometry is $\eta$-dependent ${ }^{7}, 37$ different matrices $M$ are built, one each for the 37 pseudorapidity towers in half of the calorimeter. The other half, with negative $z$-coordinates, is handled using reflection symmetry.

The matrix elements are computed using a reference sample of $N$ Monte Carlo electrons $^{8}$ with a wide range in energies $(10 \mathrm{GeV}$ to $150 \mathrm{GeV})$, and a wide range in $z_{v t x}$. For two observables $x_{i}$ and $x_{j}$, the correlation is defined as:

$$
M_{i j}=\frac{1}{N} \sum_{n=1}^{N}\left(x_{i}^{n}-\bar{x}_{i}\right)\left(x_{j}^{n}-\bar{x}_{j}\right)
$$

where $x_{i}^{n}$ is the value of the $i^{\text {th }}$ observable of the $n^{\text {th }}$ reference electron and $\bar{x}_{i}$ is the mean of the $i^{\text {th }}$ observable for the entire reference set. These matrices were verified using test beam electrons in order to ensure that they adequately describe real data.

For a particular shower, characterized by the observables $x_{i}^{\prime}$, the covariance parameter:

$$
\chi_{h m}^{2}=\sum_{i, j=1}^{41}\left(x_{i}^{\prime}-\bar{x}_{i}\right) H_{i j}\left(x_{j}^{\prime}-\bar{x}_{j}\right)
$$

is computed, where $H=M^{-1}$ is the error matrix obtained from the inverse of the correlation matrix $M$. This parameter $\chi_{h m}^{2}$ measures how closely the cluster shape is consistent with an electromagnetic shower. In general, the values of the observables $x_{i}$ are not normally distributed, and therefore the covariance parameter $\chi_{h m}^{2}$ does not

\footnotetext{
${ }^{7}$ The transverse cell size is a function of the pseudorapidity.

${ }^{8}$ Monte Carlo simulation of the calorimeter is performed using GEANT [77].
} 
follow a true $\chi^{2}$ distribution. Nevertheless, the covariance parameter offers strong rejection power against background sources since only genuine electrons will have low $\chi_{h m}^{2}$ values, as illustrated in Figure 3.3.

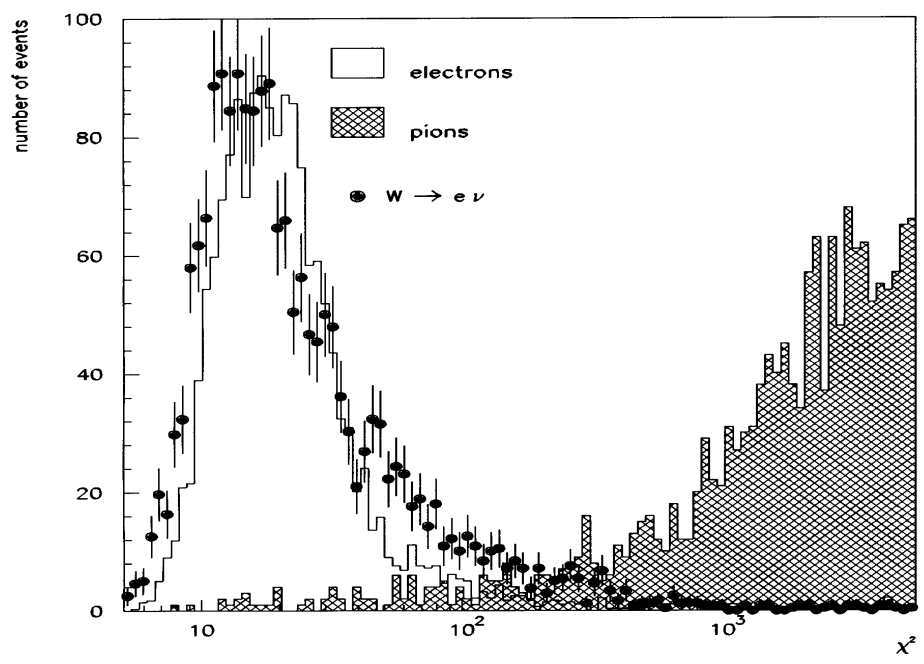

Figure 3.3: $\chi_{h m}^{2}$ distribution for test beam electrons (unshaded), test beam pions (shaded), and electrons from $W \rightarrow e \nu$ events (dots).

It is worthwhile mentioning that the $H$ matrix is a symmetric matrix, and it can be diagonalized using an appropriate unitary matrix $U$. The covariance parameter can be rewritten as:

$$
\chi_{h m}^{2}=y H^{\prime} y^{T}
$$

so that the transformed matrix $H^{\prime}=U^{T} H U$ is diagonal and the components of the vector $y$ are uncorrelated variables. These matrices, as mentioned previously, are calculated using Monte Carlo events. Slight differences in shower shapes between Monte Carlo and data can cause large contributions to $\chi_{h m}^{2}$, if the eigenvalues of the matrices are unusually large. To prevent any component from dominating the value of the covariance parameter $\chi_{h m}^{2}$, the magnitude of the diagonal elements of $H^{\prime}$ are 
limited to a maximum value, which optimizes the separation between electrons and hadrons.

In this analysis, electron candidates are required to have $\chi_{h m}^{2}<100$. The effect of this cut on electrons from $Z \rightarrow e e$ decays and electrons from multijet triggers is shown in Figure 3.4.
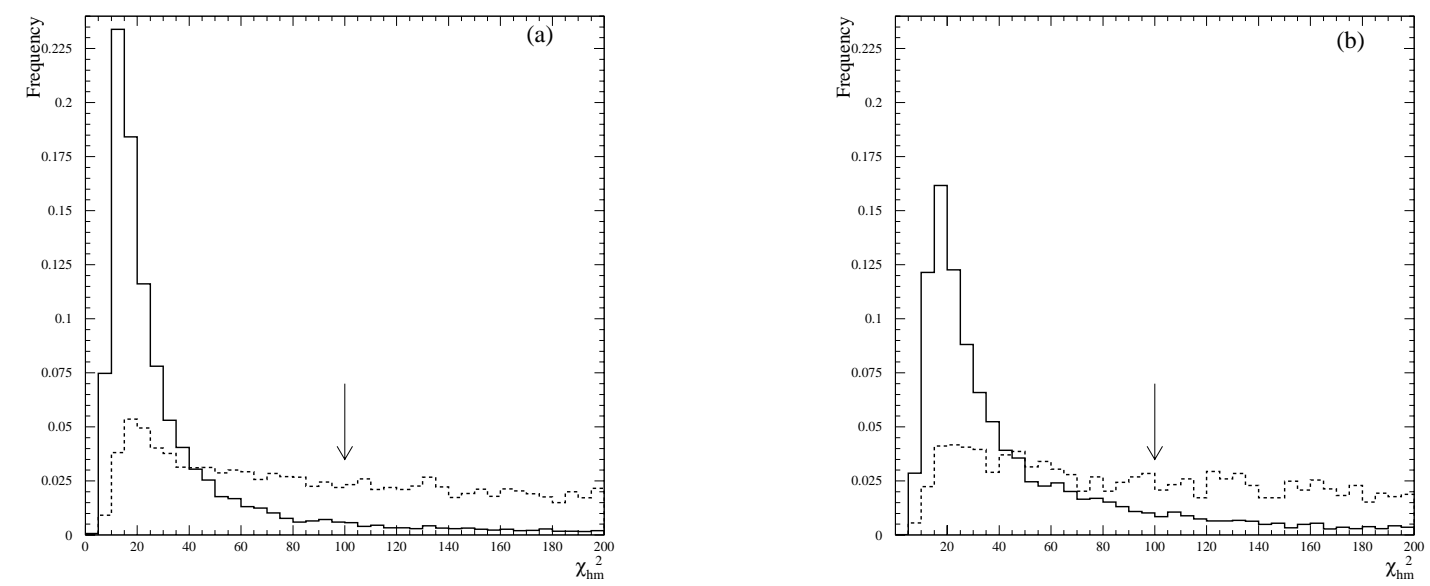

Figure 3.4: H-matrix $\chi_{h m}^{2}$ distribution for electrons from $Z \rightarrow e e$ candidates (solid) and electrons in multi-jet triggered data (dashed), for (a) central electrons and (b) forward electrons.

\subsubsection{Shower Isolation}

Electrons originating from the decays of $W$ and $Z$ bosons are isolated: very little activity surrounds the cluster since it was not produced in association with other particles. In contrast, the production of $\pi^{0}$ and $\eta$ particles (which can mimic an electromagnetic shower), or the production of electrons from heavy quark leptonic decays, that are isolated from other hadrons is relatively rare $^{9}$. Hence an isolation

\footnotetext{
${ }^{9} \mathrm{It}$ is on the order of $10^{-3}-10^{-4}$.
} 
requirement does not identify genuine eletrons, but rather selects a particular type of physics process: in this case, requiring isolated electrons preferentially selects $W$ and $Z$ events, while rejecting other sources of real electrons.

Since electromagnetic showers are usually contained in a cone of radius $\mathcal{R}=0.2$, an isolation fraction variable is defined as:

$$
f_{\text {iso }}=\frac{E_{\text {total }}(0.4)-E_{E M}(0.2)}{E_{E M}(0.2)}
$$

where $E_{\text {total }}(0.4)$ is the total energy in an isolation cone of radius $\mathcal{R}=0.4$, and $E_{E M}(0.2)$ is the electromagnetic energy in a core cone of radius $\mathcal{R}=0.2$. Distributions of the isolation variable, $f_{i s o}$, are shown in Figure 3.5 for electrons from $Z \rightarrow e e$ decays and electrons from multijet triggers. For this analyis, a requirement of $f_{\text {iso }}<0.15$ is imposed on all electron candidates.
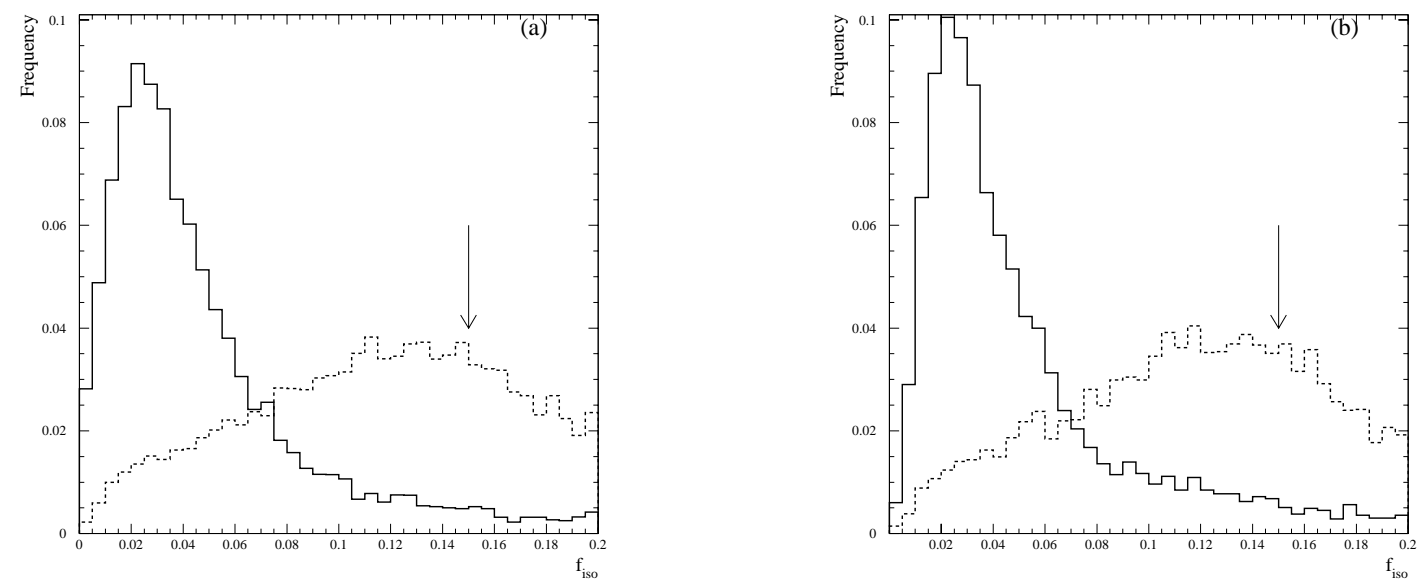

Figure 3.5: Isolation distribution $f_{\text {iso }}$ for electrons from $Z \rightarrow e e$ candidates (solid) and electrons in multi-jet triggered data (dashed), for (a) central electrons and (b) forward electrons. 


\subsubsection{Track Matching}

Electrons are defined by DØRECO as electromagnetic clusters with a track present in the road defined by the vertex position and the cluster centroid. Since the road definitions were quite loose (refer to Equation 3.28 in Section 3.6), background contamination due to accidental overlaps (such as the presence of $\pi^{0}$ or $\eta$ and additional nearby soft charged hadrons) can be substantial. The tracks of genuine electrons are expected to be well aligned with the calorimeter cluster, hence background rejection can be achieved if tighter cluster-track matching is performed than the road method of DØRECO.

To quantify the quality of the cluster-track matching, the track is extrapolated into the EM3 layer of the calorimeter and the distance between the projection and the cluster centroid is determined in both longitudinal $(\theta)$ and transverse $(\phi)$ directions. In order to place any significance on this spatial mismatch, one must understand the resolutions in track projection-cluster matching. For electrons in the central calorimeter, this resolution is $1.7 \mathrm{~cm}$ in the longitudinal direction and $0.3 \mathrm{~cm}$ in the transverse direction. For electrons in the end calorimeter, these resolutions are $0.7 \mathrm{~cm}$ and $0.3 \mathrm{~cm}$ respectively (see Figure 3.6 ). The track match significance in the central calorimeter is then defined as:

$$
S_{t r k}^{C C}=\sqrt{\left(\frac{\rho \Delta \phi}{\sigma_{\rho \phi}}\right)^{2}+\left(\frac{\Delta z}{\sigma_{z}}\right)^{2}}
$$

where $\rho \Delta \phi$ is the transverse spatial mismatch, $\Delta z$ is the longitudinal spatial mismatch, and $\sigma_{\rho \phi}$ and $\sigma_{z}$ the corresponding resolutions. Similarly, the track match 

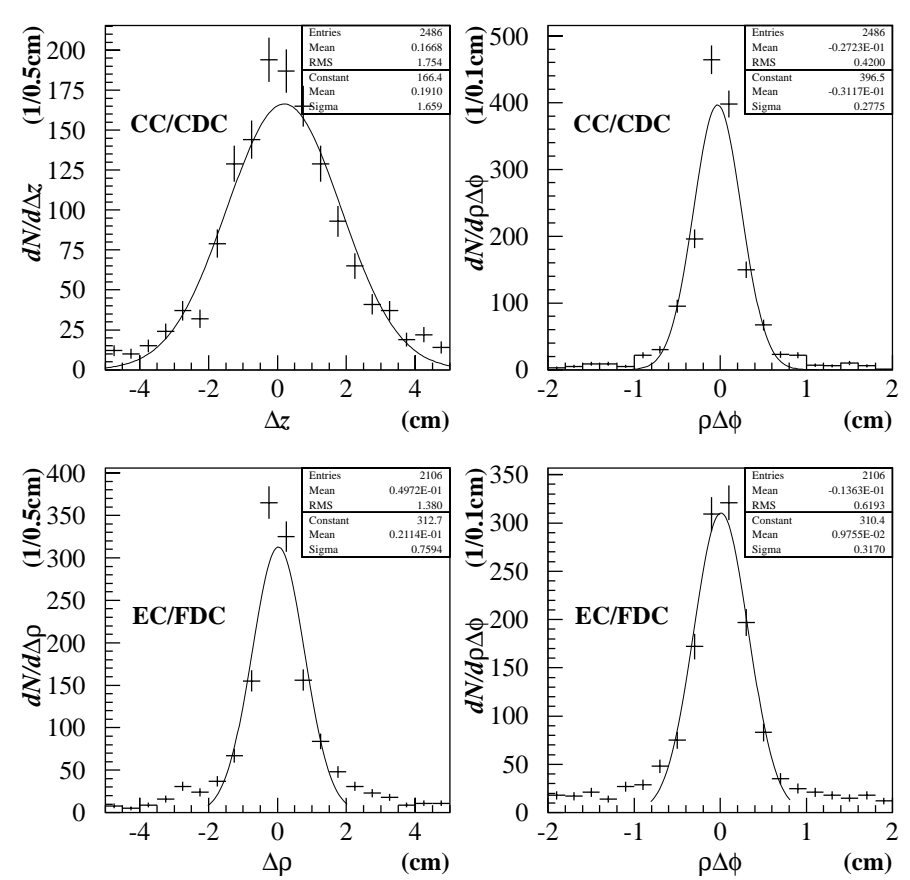

Figure 3.6: Differences in cluster centroid and EM3 projected track positions for electrons from $Z \rightarrow e e$ candidates with $S_{t r k}<30$ [68, p. 80].

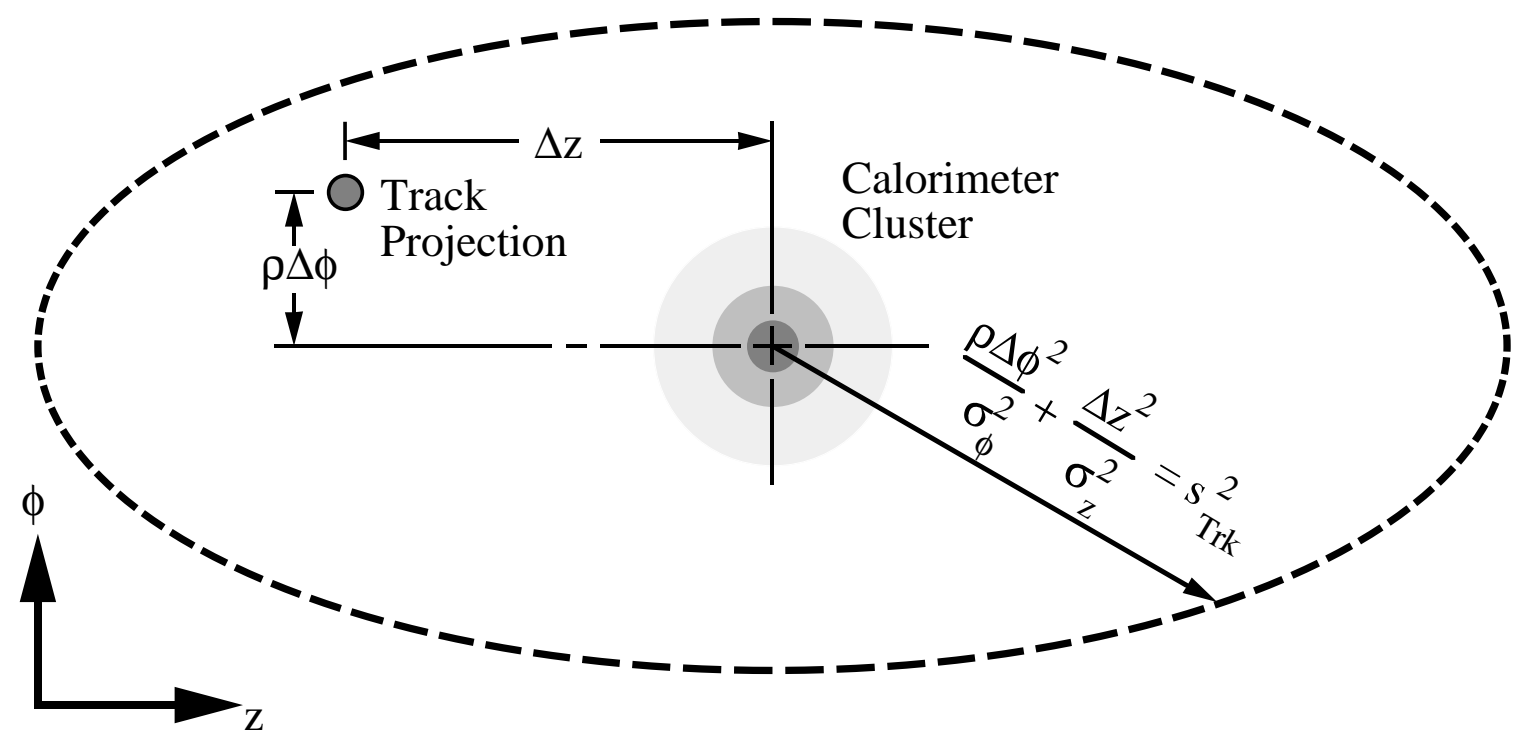

Figure 3.7: Definition of track match significance in terms of the cluster centroid in EM3 and the projection of the track to that radius [68, p. 81]. 
significance in the end calorimeter is defined as:

$$
S_{t r k}^{E C}=\sqrt{\left(\frac{\rho \Delta \phi}{\sigma_{\rho \phi}}\right)^{2}+\left(\frac{\Delta \rho}{\sigma_{\rho}}\right)^{2}}
$$

where $\rho \Delta \phi$ is the transverse spatial mismatch, $\Delta \rho$ is the longitudinal spatial mismatch, and $\sigma_{\rho \phi}$ and $\sigma_{\rho}$ the corresponding resolutions. To clarify the definition of track match significance further, an illustration of its physical meaning is shown in Figure 3.7: track projections which fall within the indicated significance ellipse projected onto the surface of the EM3 layer are considered good matches.

Distributions of the track match significance variable, $S_{t r k}$, are shown in Figure 3.8 for electrons from $Z \rightarrow e e$ decays and electrons from multijet triggers. For this analysis, the track match significance requirement was $S_{t r k}<5$ for central electrons and $S_{t r k}<10$ for forward electrons.
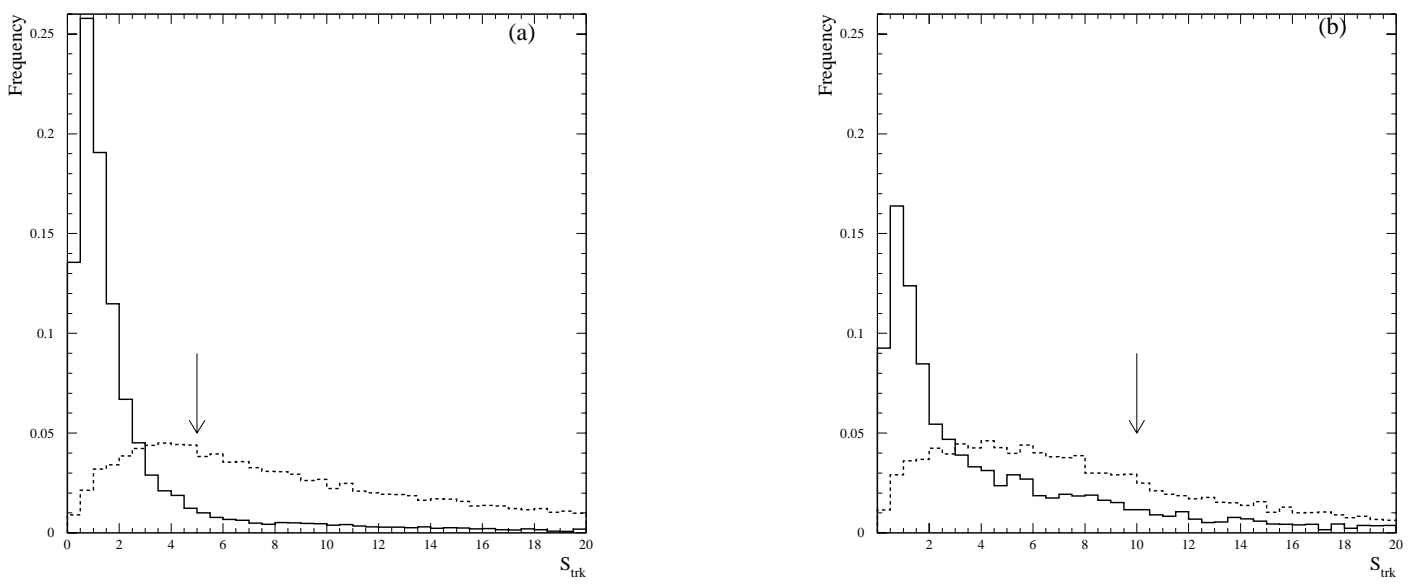

Figure 3.8: Track match siginificance $S_{t r k}$ distribution for electrons from $Z \rightarrow e e$ candidates (solid) and electrons in multi-jet triggered data (dashed), for (a) central electrons and (b) forward electrons. 


\subsection{Extension to Standard Electron ID}

The previous section described, in detail, the "standard" electron identification criteria. However, improvements can be made which result in higher efficiency, thus leading to smaller systematic errors. The extended technique used in this analysis makes use of tracking information to improve the determination of the interaction vertex.

As mentioned in Section 3.6, the distinction between electron candidates and photon candidates relies on the presence (or lack thereof) of a track in the road defined by the calorimeter cluster and the primary vertex. Mismeasurement of the vertex position leads to an incorrect definition of the tracking road, which in turn leads to a possible misidentification of electrons as photons. In addition, important kinematic quantities (such as electron $E_{T}$, gauge boson invariant mass, etc.) which depend on the vertex position will be affected as well. A method was developed which attempts to identify the primary vertex by using the electron information, and will be described in the following section.

\subsubsection{Electron Vertex Finding}

The electron vertex finding algorithm [68, sec. 4.8.1] relies on calorimeter cluster and associated track matching, instead of tracking roads. For a given electromagnetic cluster in the calorimeter, a search is performed for the best matching track, regardless of whether this track was contained in the tracking road. This search is performed 


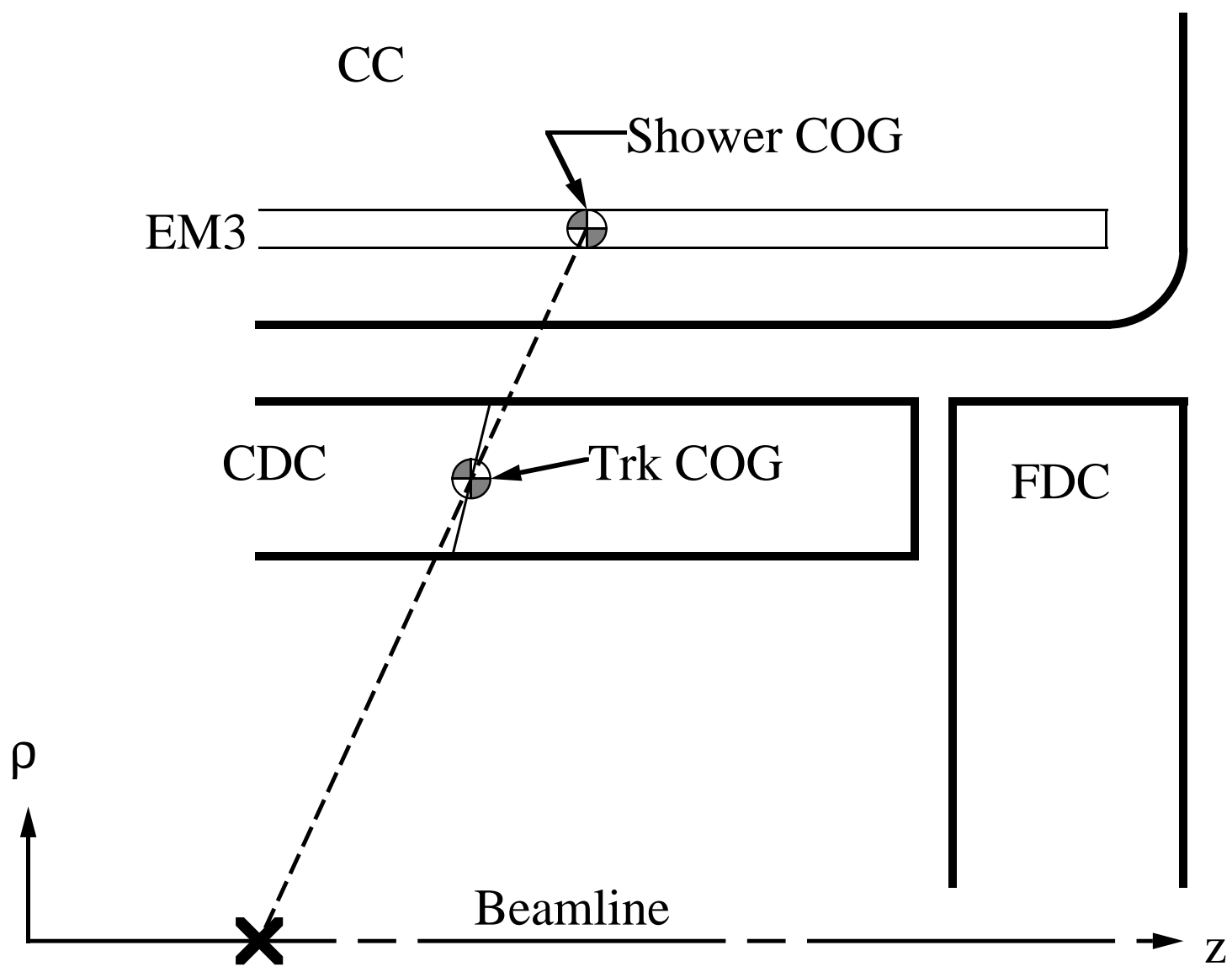

Figure 3.9: Vertex determination by electron cluster-track projection method [68, p. 84].

on all CDC and FDC tracks ${ }^{10}$, and track match significance is computed exactly as described in Equation 3.33 and Equation 3.34. This track can then be used to determine the origin of the electron by extrapolating the line connecting the calorimeter cluster's center-of-gravity and the track's center-of-gravity to the beamline. Hence, the $z$-coordinate of the interaction vertex, denoted as $z_{v}$, is given by:

$$
z_{v}=z_{0}^{t r k}-\left(\frac{z_{0}^{c a l}-z_{0}^{t r k}}{\rho_{0}^{c a l}-\rho_{0}^{t r k}}\right) \rho_{0}^{t r k}
$$

where $\left(z_{0}^{\text {trk }}, \rho_{0}^{\text {trk }}\right)$ and $\left(z_{0}^{c a l}, \rho_{0}^{c a l}\right)$ are the centers-of-gravity of the drift chamber track and the calorimeter cluster respectively. This extrapolation is illustrated in Figure 3.9

\footnotetext{
${ }^{10}$ VTX chamber tracks are ignored.
} 

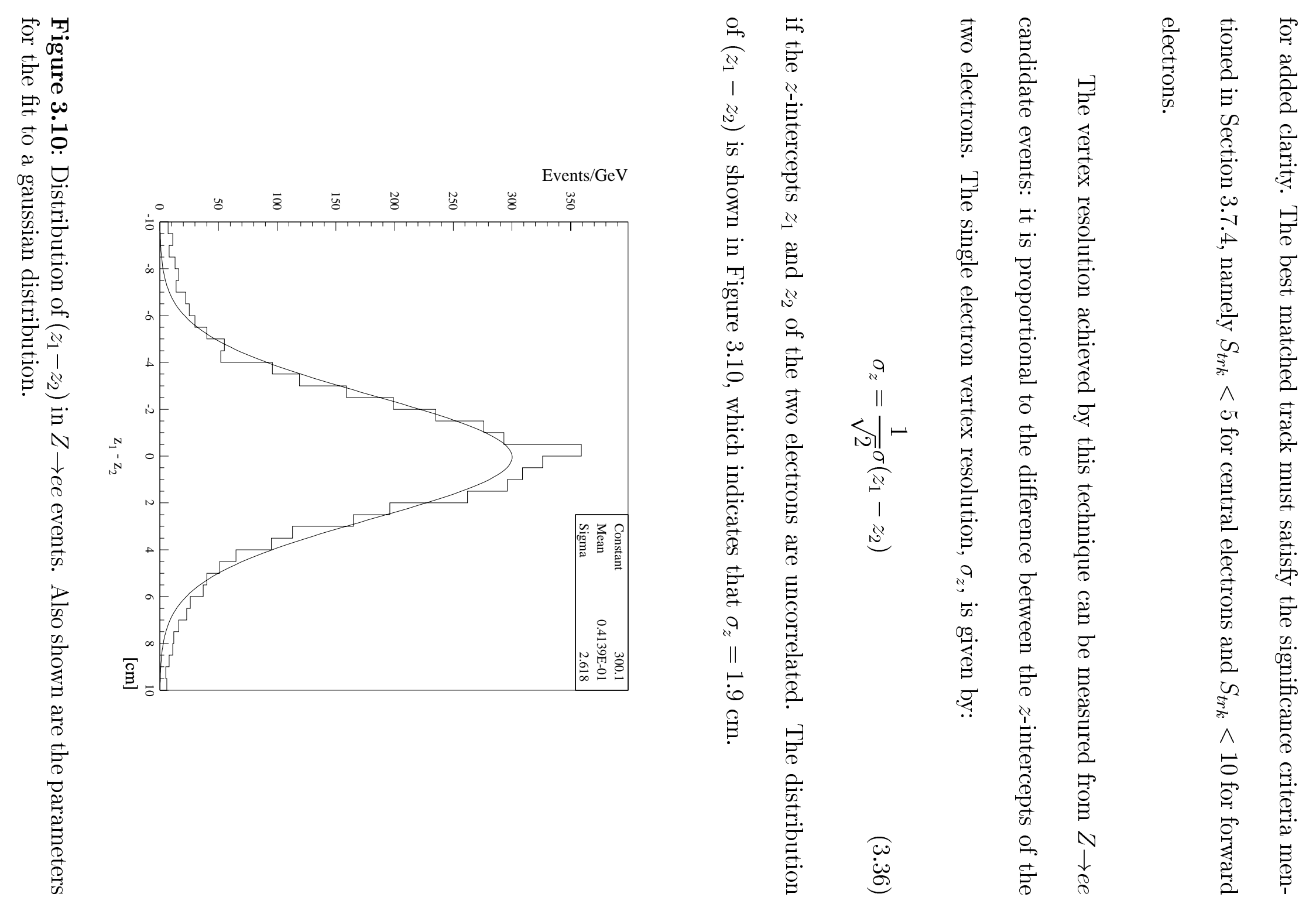


\subsubsection{Performance of Electron Vertex Finding}

The performance of the electron vertex finding algorithm can be compared to the standard algorithm in DØRECO. In fact, with the knowledge of the single electron vertex resolution, it is possible to measure how often DØRECO misreconstructs the primary vertex position. The standard vertex is considered to be mismeasured if it is at least 5 standard deviations from the single electron vertex (in this case, this distance amounts to $10 \mathrm{~cm}$ ). As is shown in Figure 3.11a, the rate at which this occurs in $Z \rightarrow e e$ events $^{11}$ grows as a function of instantaneous luminosity. For the inclusive $Z \rightarrow e e$ sample, about $13 \%$ of the events have mismeasured primary vertices. In contrast, the rate at which $\left(z_{1}-z_{2}\right)>10 \mathrm{~cm}$ is much flatter as a function of instantaneous luminosity, indicating that the electron vertex algorithm is quite robust. This situation would have been quite different if the busy environment in high luminosity events was affecting the electron vertex algorithm via random overlaps and/or reconstruction inefficiencies.

In addition, the invariant mass spectrum of $Z \rightarrow e e$ candidate events is shown in Figure 3.11b for both vertexing algorithms. It is clearly visible that the electron vertex algorithm increases the number of events in the central peak region. The broader distribution in the standard vertex algorithm is caused by misreconstructed interaction vertices which lead to a mismeasurement of the invariant mass of the two electrons $^{12}$.

\footnotetext{
${ }^{11}$ In $Z \rightarrow e e$ events, the single electron vertex position is determined by the most central electron which has a matching track. The most central electron is the one nearest $\eta_{\text {det }}=0$.

${ }^{12}$ This is caused by a mismeasurement of the opening angle between the two electrons.
} 


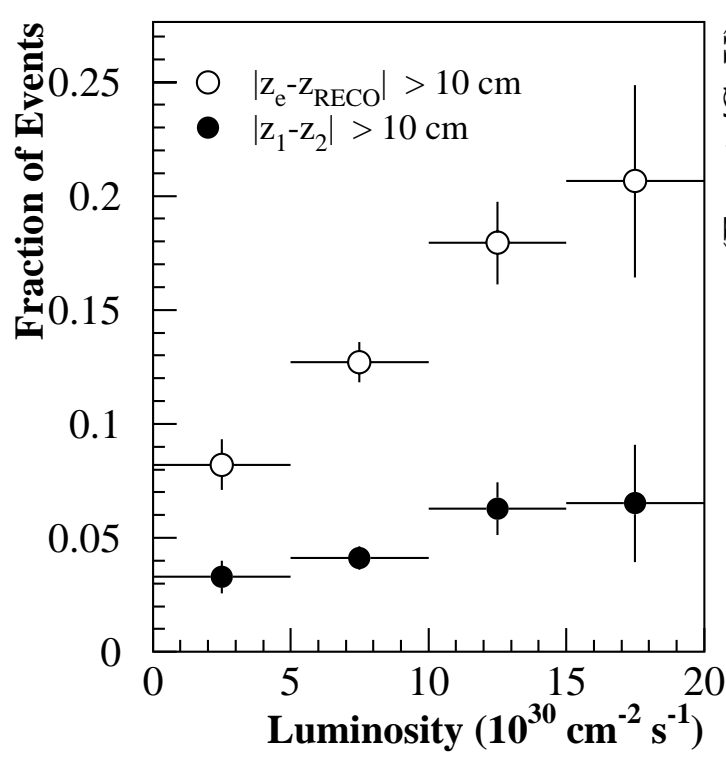

(a)

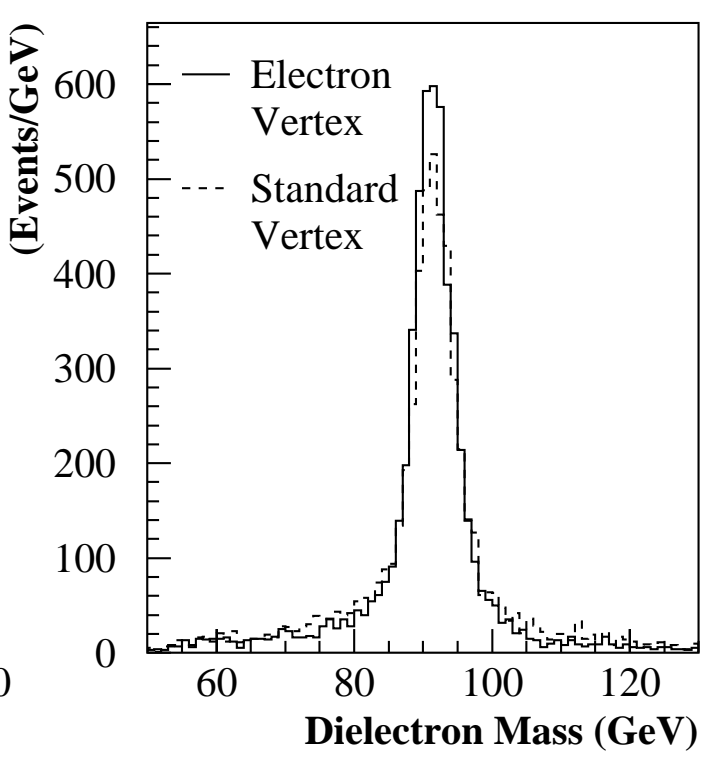

(b)

Figure 3.11: (a) Frequency at which the standard vertex is mismeasured as a function of instantaneous luminosity. The standard vertex is considered to be mismeasured if it is found more than $10 \mathrm{~cm}$ away from the electron vertex. As a benchmark, the rate at which the electron $z$-intercepts differ by more than $10 \mathrm{~cm}$ is also shown. (b) Invariant mass distribution for $Z \rightarrow e e$ events using the two vertexing algorithms [68, p. 86].

\subsection{Neutrino Identification}

$W$ bosons decay into an electron and a neutrino. As mentioned previously, the presence of the neutrino is inferred from $\mathbb{E}_{T}$, the overall imbalance in the transverse energy deposited in the calorimeter. In fact, the transverse momentum of the neutrino, $p_{T}^{\nu}$, is defined as the magnitude of $\mathscr{E}_{T}$. The calculation of $\not_{T}$ from the individual cells in the calorimeter has already been described (Section 3.4). However, most analyses apply corrections to the energies of various physics objects, such as electrons, jets, or muons which then must be incorporated into the calculation of $\mathbb{E}_{T}$. 
For these analyses, it is important to clearly define what the energy corrections are, and how they get propagated into the calculation of $\mathbb{E}_{T}$. In this analysis, corrections are applied to the signal electron(s) in $W$ and $Z$ events.

All electrons are corrected by the standard electromagnetic correction. The calibration of the EM calorimeter cryostats was provided initially by test beam energy calibrations using electrons and pions. This calibration was already included in the determination of the energy deposited in each cell. However, when one studies a clean sample of $Z \rightarrow e e$ events, one finds that the fitted $Z$ boson mass is a few percent lower than its world average value [18]. Extensive studies [78, ch. 6] of the absolute calibration of the EM calorimeter finds that this deficit is mostly due to a scale factor, with an additional offset that is close to zero. Based on this, multiplicative corrections are derived for each EM cryostat separately, and are summarized in Table 3.1. The reader is referred to [79] for the reasons underlying this miscalibration. In addition, the electron vertexing algorithm introduces angular corrections to the electrons, which affect their transverse energy determination.

Table 3.1: EM scale factors applied to each calorimeter cryostat.

\begin{tabular}{cc}
\hline \hline Cryostat & EM correction factor \\
\hline CC & 1.0502 \\
EC South & 1.0454 \\
EC North & 1.0639 \\
\hline \hline
\end{tabular}

The final calculation of the missing transverse energy starts with the calorimeter based $\mathbb{E}_{T}$, with ICD and massless gap corrections. The scalar quantity $\mathbb{E}_{T}$ from 
Equation 3.14, along with the azimuthal angle $\phi_{E_{T}}$ from Equation 3.15 are used to calculate the $x$ and $y$ components of the missing transverse energy vector. As each energy correction is applied, the transverse components of the correction vector are also computed. These in turn are propagated ${ }^{13}$ into the components of $\mathbb{E}_{T}$ :

$$
\begin{aligned}
& \mathscr{E}_{x}{ }^{\prime}=E_{x}+\sum_{i} \Delta E_{x}^{i} \\
& \mathscr{E}_{y}{ }^{\prime}=E_{y}+\sum_{i} \Delta E_{y}^{i} .
\end{aligned}
$$

where the index $i$ labels all the corrections that are applied. Hence the final $\mathbb{E}_{T}$ magnitude and direction are:

$$
\mathscr{E}_{T}^{\prime}=\sqrt{\left(\mathscr{E}_{x}^{\prime}\right)^{2}+\left(\mathbb{E}_{y}^{\prime}\right)^{2}}
$$

and

$$
\phi_{E_{T}}^{\prime}=\arctan \left(\frac{\mathbb{E}_{y}^{\prime}}{\mathbb{E}_{x}^{\prime}}\right) .
$$

The prescription for the calculation of $\mathbb{E}_{T}$ outlined above ignores any corrections made to muons and jets found in the event. This leads to a small degradation in the $\mathscr{E}_{T}$ resolution. This is acceptable as long as the same prescription is followed in modeling the $\mathbb{E}_{T}$ resolution (see Chapter 5).

\footnotetext{
${ }^{13}$ Recall that $\vec{H}_{T}=-\vec{E}_{T}^{t o t}$.
} 


\section{Chapter 4}

\section{Event Samples}

The Fermilab Tevatron started its physics collider run in late 1992, and operated for a period of three and one half years, until early 1996. This run, known as Run I, consists of three distinct periods: Ia, Ib, and Ic. DØ collected data during all three periods, for a total integrated luminosity of $\sim 110 \mathrm{pb}^{-1}$. However, the data used in this analysis were taken during Run Ib, from February 1994 to April 1995, and Run Ic, from November 1995 to March 1996. The integrated luminosity over these two periods amounts to roughly $95 \mathrm{pb}^{-1}$, which is approximately six times the amount of data collected in Run Ia. The history of data taking at DØ is illustrated in Figure 4.1. The difference between delivered and recorded luminosity is caused by detector downtime (9\% of the delivered luminosity) or by beam conditions (see Section 2.7.2.1) that are not suitable for physics analyses (13\% of the delivered luminosity).

This chapter deals with the selection of $W \rightarrow e \nu$ and $Z \rightarrow e e$ event candidates from Run Ib and Ic. Nearly all the physics data is used for this analysis: a small amount $(<1 \%)$ is left out if it had originated from runs with known detector problems. 


\section{$D \varnothing$ Run I Integrated Luminosity}

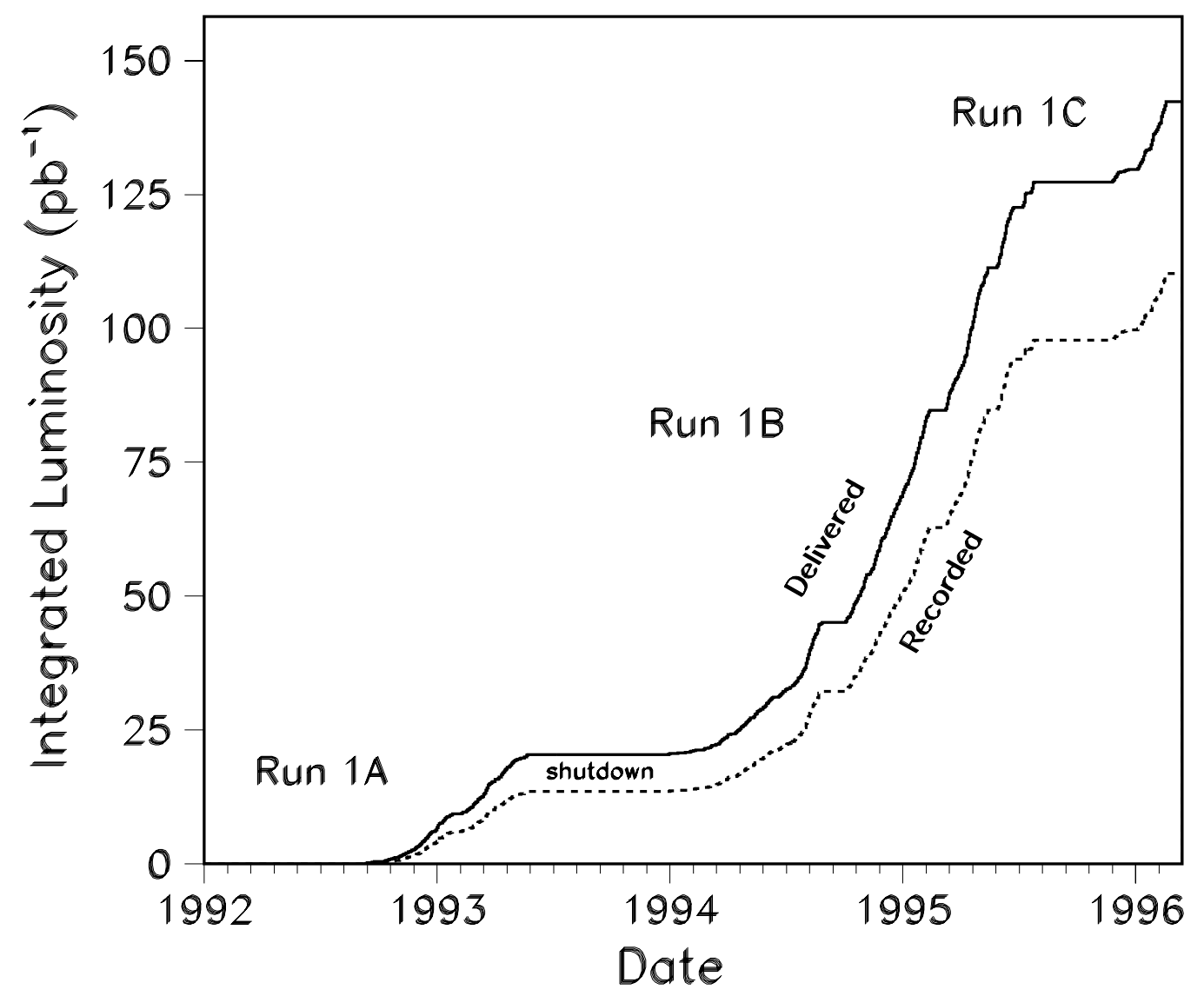

Figure 4.1: The Run I integrated luminosity as a function of time. The difference between delivered and recorded luminosity is due to Main Ring beam activity and/or detector down-time. 


\subsection{Offline Electron Selection}

Before proceeding to the selection of $W$ and $Z$ events, a description of the electron selection itself must be done. Two classifications are used to describe the signal electrons: a loose selection which identifies "loose" electrons and a tight selection which identifies "tight" electrons. The tight electrons form a subset of the loose ones. The fiducial region is selected such that non-instrumented or poorly instrumented regions of the detector are eliminated. These regions include the inter-cryostat detector (ICD), the very forward regions where the segmentation of the EM calorimeter decreases, and the boundaries between the electromagnetic central calorimeter (CCEM) modules. The fiducial region is identical for tight and loose electrons:

- Central Calorimeter: $\quad\left|\eta_{\text {det }}\right|<1.1 \quad$ and $0.05<\bmod \phi_{\text {cluster }}^{e}<0.95$;

- Endcap Calorimeter: $1.5<\left|\eta_{\text {det }}\right|<2.5$.

The variable $m o d \_\phi_{\text {cluster }}^{e}$ is defined as the $\phi$-angle of the electron cluster relative to the edge of the CCEM calorimeter module, in units of the angle subtended by the module:

$$
\text { mod_ } \phi_{\text {cluster }}^{e}=\operatorname{MOD}\left(\frac{32}{2 \pi} \phi_{\text {cluster }}, 1\right)
$$

The fiducial region represents $\mathrm{a} \sim 25 \%$ loss in acceptance of the full calorimeter solid angle.

With the above fiducial region in mind, one can proceed to describe the actual criteria of tight and loose electrons: 
- $\underline{\text { Tight electron }}$

- EM cluster in the good fiducial region;

- H-matrix $\chi_{h m}^{2}<100$;

- EM fraction $f_{e m}>0.95$

- Isolation fraction $f_{\text {iso }}<0.15$

- A matching central detector track with significance $S_{\text {trk }}<5(10)$ in the CC(EC).

- $\underline{\text { Loose electron }}$

- EM cluster in the good fiducial region;

- H-matrix $\chi_{h m}^{2}<100$;

- EM fraction $f_{e m}>0.95$

- Isolation fraction $f_{\text {iso }}<0.15$.

Loose and tight electrons share all calorimeter-based electron identification criteria. However, loose electrons are not required to have a matching track. This class of electrons is only used in $Z \rightarrow e e$ event selection in order to increase the statistics of the $Z$ sample. 


\section{2 $W \rightarrow e \nu$ Event Selection}

$W \rightarrow e \nu$ candidates are selected using the signature of an isolated high- $p_{T}$ electron and a high- $p_{T}$ neutrino. The neutrino's presence is inferred by the detection of substantial $\mathbb{E}_{T} . W \rightarrow e \nu$ event selection occurs in two stages: trigger and offline. The trigger requirements are always very loose: the cuts are made in order to reduce the amount of data written to tape to an acceptable level ( see Section 2.7). The offline cuts are imposed in a such a manner as to optimize the signal to background ratio (see Section 3.7): the final candidate sample is as large as possible thus ensuring a small statistical error, while keeping the level of background to an acceptably small (yet reliably measurable) amount. Discussion on the amount of background which contaminates the final $W \rightarrow e \nu$ sample is deffered until Chapter 7 .

\subsection{1 $W \rightarrow e \nu$ Trigger Requirements}

The $W \rightarrow e \nu$ data sample was collected with the EM1_EISTRKCC_MS trigger. This trigger was configured in two different ways during Run Ib, the change occuring with the introduction of the Calorimeter Level-1.5 trigger halfway through the run (trigger configuration menu version 10.0). In addition, the trigger was completely dropped in Run Ic, since the additional gain in statistics was not deemed worthwhile ${ }^{1}$. The EM1_EISTRKCC_MS trigger had the following conditions:

\footnotetext{
${ }^{1}$ In Run Ic, the collaboration elected to focus its resources on the search for hadronically decaying $W$ bosons, $W \rightarrow q \bar{q}^{\prime}$, which required considerable trigger bandwidth.
} 
- $\underline{\text { Level- } \varnothing \text { trigger (hardware) }}$

- The universal Level- $\varnothing$ minimum bias requirement was imposed for trigger versions $<10.0$ : this consisted of the detection of an inelastic collision with simultaneous hits in the north and south Level- $\varnothing$ counters, as well as a fast $z$ determination with $|z|<96.875 \mathrm{~cm}$;

- The requirement was removed for trigger versions $\geq 10.0$. It operated in a mark and pass mode, where the Level- $\varnothing$ requirement was checked but the result was not used in the trigger decision. However, this result was recorded so that the cut may be studied and imposed offline (this issue will be discussed in Section 6.3.1).

- Level-1 trigger (hardware)

- $E_{T}^{e m}>12.0$ or $10.0 \mathrm{GeV}$. The threshold changed with trigger version 10.0 ;

- GOOdCAL beam veto: events which occured during the MRBS LOSS window were rejected.

- Level-1.5 trigger (hardware)

$-E_{T}^{e m}>15.0 \mathrm{GeV}$ (this cut was introduced with trigger version 10.0);

$-f_{e m}>0.85$ (this cut was introduced with trigger version 10.1).

- Level-2 filter (software)

$-E_{T}>20.0 \mathrm{GeV}$ 
- Loose shower shape (ele) and isolation fraction (iso) cuts

$$
\begin{array}{r}
(\text { eis } \equiv \text { ele } \cdot i s o) ; \\
- \text { EtT }_{T}>15.0 \mathrm{GeV} .
\end{array}
$$

\subsection{2 $W \rightarrow e \nu$ Offline Selection}

The final selection of $W \rightarrow e \nu$ events is performed from all Run Ib data, after runs with known problems are removed [80]. The following cuts are used:

- Event must pass EM1_EISTRKCC_MS trigger;

- Event must pass the Level- $\varnothing$ minimum bias requirement (see Section 4.2.1);

- Event must pass the GoOdBeAm veto condition: events occurring in the MRBS_LOSS or the MICRO_BLANK periods are rejected;

- One tight triggered electron with $E_{T}>25 \mathrm{GeV}$;

- the vertex for the event is defined by this electron. The z-coordinate of the vertex must have $|z|<96.875 \mathrm{~cm}$ in order to match the Level- $\varnothing$ requirement;

- Corrected $\not_{T}>25 \mathrm{GeV}$;

- Events containing a second loose electron with $E_{T}>25 \mathrm{GeV}$ are excluded (to minimize the $Z \rightarrow e$ background with mismeasured $\left.\mathbb{E}_{T}\right)$. 
Table 4.1: Summary of the $W \rightarrow e \nu$ signal event sample and topological breakdown.

\begin{tabular}{cc}
\hline \hline$W \rightarrow e \nu$ Signal Events \\
\hline $\mathrm{CC}$ & 47004 \\
$\mathrm{EC}$ & 20402 \\
\hline Total & 67406 \\
\hline \hline
\end{tabular}

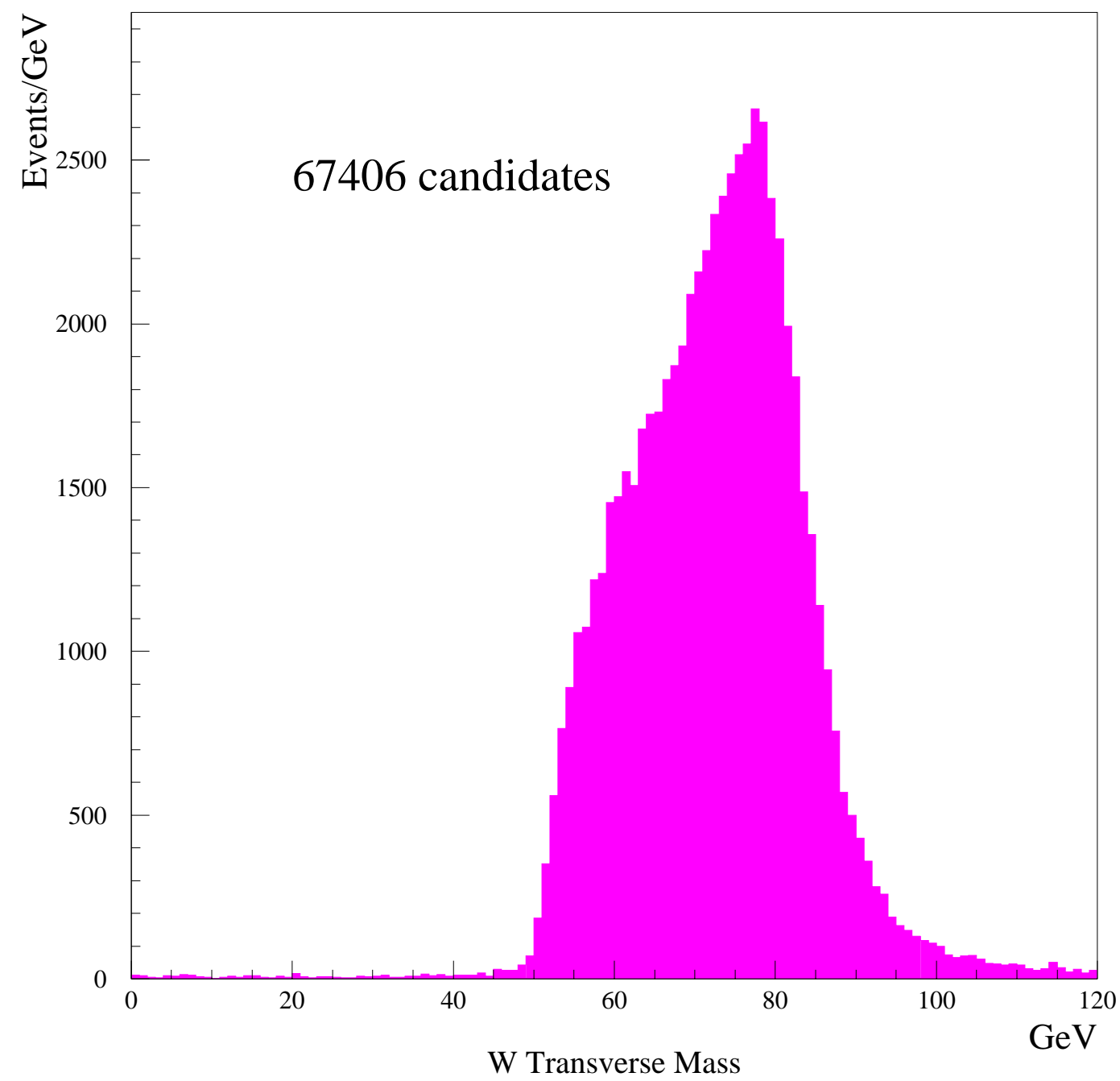

Figure 4.2: Transverse mass of the final $W \rightarrow e \nu$ candidate sample. 
A total of 67,406 events pass these cuts. The topological breakdown into central (CC) or forward (EC) events is summarized in Table 4.1. The transverse mass distribution of the candidate events is shown in Figure 4.2.

\section{3 $Z \rightarrow e e$ Event Selection}

$Z \rightarrow e e$ candidates are selected using the signature of two isolated high- $p_{T}$ electrons. The actual selection proceeds along similar lines to the $W$ case, namely with two stages: trigger and offline. Discussion on the amount of background which contaminates the final $Z \rightarrow e e$ sample is also deferred until Chapter 7 .

\subsection{1 $Z \rightarrow e e$ Trigger Requirements}

The $Z \rightarrow e$ data sample was collected with the EM2_EIS2_HI trigger. This trigger was also configured in two different ways during Run Ib, the change occuring with trigger version 10.0. Unlike the $W \rightarrow e \nu$ case, this trigger was kept during Run Ic since $Z \rightarrow e e$ event samples are statistically limited and their use goes beyond a mere $Z \rightarrow e e$ cross section analysis: they are invaluable as a calibration tool in understanding the detector. The EM2_EIS2_HI trigger had the following conditions:

- Level- $\varnothing$ trigger

- The universal Level- $\varnothing$ minimum bias requirement was imposed throughout the data taking period. 
- $\underline{\text { Level-1 trigger }}$

-2 EM objects with $E_{T}^{e m}>7.0 \mathrm{GeV}$;

- MAXLive beam veto: events occurring in the MRBS_LOSS and MICRO_BLANK periods simultaneously, were rejected.

- Level-1.5 trigger

-2 EM objects with $E_{T}^{e m}>12.0 \mathrm{GeV}$ (trigger version $\geq 10.0$ );

-2 EM objects with $f_{\text {em }}>0.85$ (trigger version $\geq 10.1$ ).

- $\underline{\text { Level-2 filter }}$

- 2 EM objects with $E_{T}>20.0 \mathrm{GeV}$;

- Loose shower shape and isolation fraction cut (eis) on both objects.

\subsection{2 $Z \rightarrow e e$ Offline Selection}

The final selection of $Z \rightarrow e e$ events is performed from all Run Ib and Ic data, after runs with known problems are removed [80]. The following cuts are used:

- Event must pass the EM2_EIS2_HI trigger;

- Two loose triggered electron with $E_{T}>25 \mathrm{GeV}$ each, one of which must be tight; 
Table 4.2: Summary of the $Z \rightarrow e e$ signal event sample and topological breakdown.

\begin{tabular}{cc}
\hline \hline$Z \rightarrow e e$ Signal Events \\
\hline CC-CC & 3628 \\
CC-EC & 2831 \\
EC-EC & 681 \\
\hline Total & 7140 \\
\hline \hline
\end{tabular}

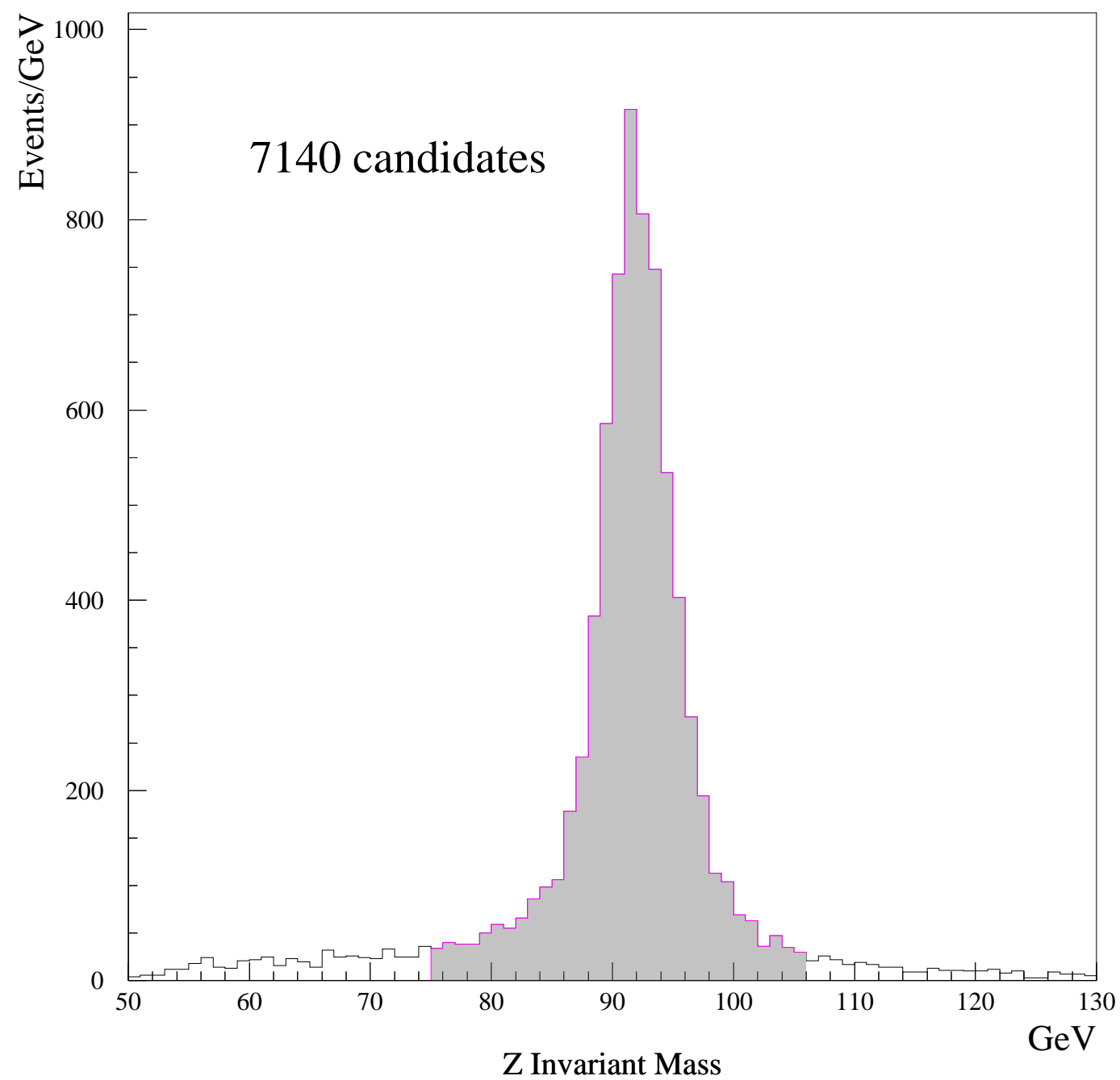

Figure 4.3: Invariant mass of the final $Z \rightarrow e e$ candidate sample. 
- the vertex for the event is defined by the tight electron. In case both electrons are tight, the most central (smallest $\left|\eta_{\text {det }}\right|$ ) is used to define the vertex. The $z$-coordinate of the vertex must have $|z|<96.875 \mathrm{~cm}$ in order to match the Level- $\varnothing$ requirement;

- Invariant mass of the dielectron pair: $75<M_{e e}<105 \mathrm{GeV} / \mathrm{c}^{2}$.

A total of 7,140 events pass these cuts. The topological breakdown into centralcentral (CC-CC), central-forward (CC-EC), and forward-forward (EC-EC) events is summarized in Table 4.2. The invariant mass distribution of the candidate events is shown in Figure 4.3. 


\section{Chapter 5}

\section{Detector Acceptance}

The processes $p \bar{p} \rightarrow W \rightarrow e \nu$ and $p \bar{p} \rightarrow Z \rightarrow e e$ are identified at $\mathrm{D} \varnothing$ by the decay leptons. However, only a fraction of the events that are produced end up being observed. Electrons might escape detection if they enter an uninstrumented region of the detector, or if they fail to satisfy the fiducial criteria. Furthermore, the decay leptons might not possess sufficient transverse momentum to satisfy the selection criteria.

A fast Monte Carlo simulation, CMS [78][81], is used to generate $W$ and $Z$ bosons, perform their decay, and apply the detector effects to the decay products. The acceptance of $W \rightarrow e \nu$ and $Z \rightarrow e e$ events produced in $p \bar{p}$ collisions is then defined as the ratio of the number of events which pass the fiducial and kinematic requirements to the total number of events generated. A brief desciption of CMS is provided for completeness, but the reader is urged to consult [78, chap. 5] and [81, chap. 3] for additional details. 


\subsection{Event Generation}

Vector boson production is ideally modeled with a a fully differential cross section:

$$
\frac{d^{5} \sigma}{d m d p_{T} d y d \phi d \epsilon}
$$

where $m, p_{T}, y, \phi$, and, $\epsilon$ are the vector boson mass, transverse momentum, rapidity, azimuthal angle, and polarization, respectively. In the CMS Monte Carlo, this differential cross section is factored into four separate pieces:

$$
\frac{d^{5} \sigma}{d m d p_{T} d y d \phi d \epsilon}=\frac{d \sigma}{d m} \cdot \frac{d^{2} \sigma}{d p_{T} d y} \cdot \frac{d \sigma}{d \phi} \cdot \frac{d \sigma}{d \epsilon}
$$

The $\phi$ distribution is trivial: $\frac{d \sigma}{d \phi}$ is uniform, hence a value is chosen at random in the interval $[0,2 \pi)$. The factorization of the three remaining terms is not strictly correct, since correlations between them do exist. Fortunately, these correlations have a negligible effect on the acceptance.

\subsection{1 $W$ and $Z$ Boson Generation}

In the generation of $Z$ bosons, the polarization vector is chosen randomly to lie either in the direction of the incoming proton, or opposite to it. For the $W$ boson case, the charge defines its polarization. For a $W^{+}$, the polarization vector is opposite the proton direction ${ }^{1}$. For the fraction of events involving quarks originating from the sea $\left(f_{s s} \approx 20 \%\right)$, one half of these events have their polarization reversed.

\footnotetext{
${ }^{1}$ The proton direction is taken to be along the positive $z$-axis (see Section 2.2).
} 
The mass of the boson is selected from a relativistic Breit-Wigner (with a $\hat{s}^{-}$ dependent width) modified by a parton luminosity term. This term is introduced to model the dependence of the mass on the momentum distribution of the quarks: this distribution favors the production of vector bosons with a lower mass. The mass distribution is then given by:

$$
\frac{d \sigma}{d m}=P L(m) \cdot \frac{m}{\left(m^{2}-M^{2}\right)^{2}+m^{4},{ }^{2} / M^{2}}
$$

where $P L(m)$ is the parton luminosity term, $m$ is the mass of the vector boson being generated, and $M$ and, are the boson's true mass and natural width, respectively. The parton luminosity term is dependent on the choice of stucture function. It is found to be well modeled by:

$$
P L(m)=\frac{e^{-\beta \cdot m}}{m}
$$

where the parton luminosity slope $\beta$ is obtained by fitting the vector boson invariant mass distribution generated from the HERWIG Monte Carlo [82] to the function expressed in Equation 5.3 (see Figure 5.1).

\subsubsection{Transverse Momentum and Rapidity}

At tree level, $W$ and $Z$ production is based on the Drell-Yan process (see Figure 5.2), where the boson is generated through $q \bar{q}$ annihilation. The longitudinal momentum of the boson is determined by the momentum imbalance of the incoming quarks. Since the vector boson is the only final state particle, it is generated without any transverse momentum. However, higher order QCD processes allow final states 


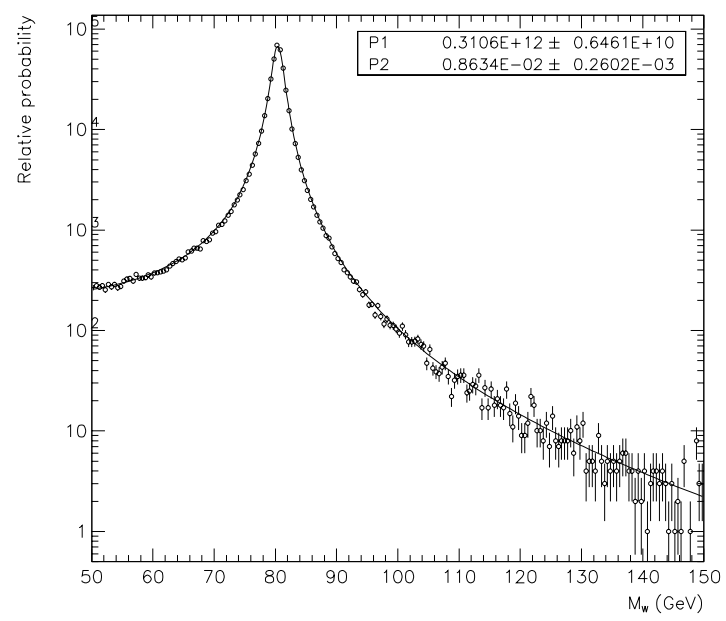

Figure 5.1: Mass distribution from HERWIG showing the parton luminosity effect [81, p. 68].

in which hadrons may recoil against the boson, thus providing it with transverse momentum. Such processes include initial state gluon radiation and the Compton process (see Figure 5.3). As an alternative to incorporating such higher order processes into the boson generation itself, the generated $W$ and $Z$ bosons are given transverse momentum according to theoretical calculations which include perturbative, as well as non-perturbative contributions. In the high- $p_{T}$ regime $\left(p_{T}>50 \mathrm{GeV}\right)$, the second order perturbative calculation of Arnold and Reno [83] is used. For the low- $p_{T}$ regime $\left(p_{T}<50 \mathrm{GeV}\right)$, the resummed calculation of Ladinsky and Yuan [84] is used. Resummation [85] is the theoretical framework which involves rearraging the divergent logarithms that appear in the perturbation series, and summing them into an exponential factor. 
$\mathrm{u}$

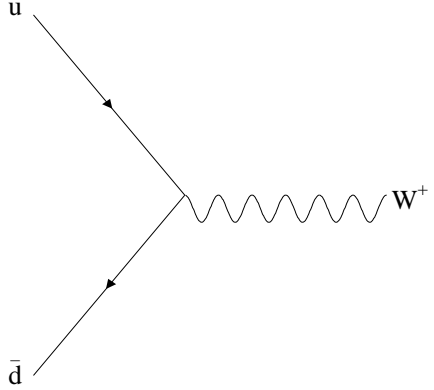

$\mathrm{u}$

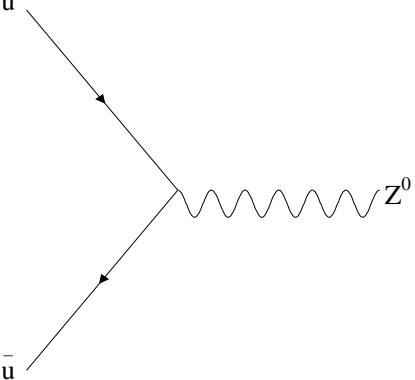

Figure 5.2: Lowest order (Drell-Yan) diagrams for $W$ and $Z$ production.

The resummed double differential cross section for vector boson production is written as:

$$
\frac{d^{2} \sigma}{d p_{T} d y} \propto \int \frac{d^{2} b}{(2 \pi)^{2}} e^{i \bar{b} \cdot \bar{p}_{T}} W\left(b_{\star}\right) e^{-S_{N P}(b)}
$$

where $b$, the impact parameter in the transverse plane, is the conjugate variable to $p_{T}$ : small values of $b$ correspond to large $p_{T}$ and large $b$ to low $p_{T}$. $b_{\star}$ is a function of $b$ which handles the divergence at high- $b$ values by incorporating a cutoff $b_{\max }$ :

$$
b_{\star}=\frac{b}{\sqrt{1+b^{2} / b_{\max }^{2}}} .
$$

The function $W\left(b_{\star}\right)$ is a complicated but well defined function calculated in perturbation theory [83], while the function $S_{N P}(b)$ incorporates the non-perturbative effects at high- $b$ values obtained from the resummation. Ladinsky and Yuan use the following parametrization for $S_{N P}$ :

$$
S_{N P}=g_{1} b^{2}+g_{2} b^{2} \ln \left(\frac{Q}{2 Q_{\circ}}\right)+g_{1} g_{3} \ln \left(100 x_{A} x_{B}\right)
$$

with $Q_{\circ}$ an arbitrary momentum scale, $Q$ the mass of the vector boson, and $x_{A}$, $x_{B}$ the momentum fractions of the incoming quarks. The parameters $g_{1}, g_{2}$, and $g_{3}$ 

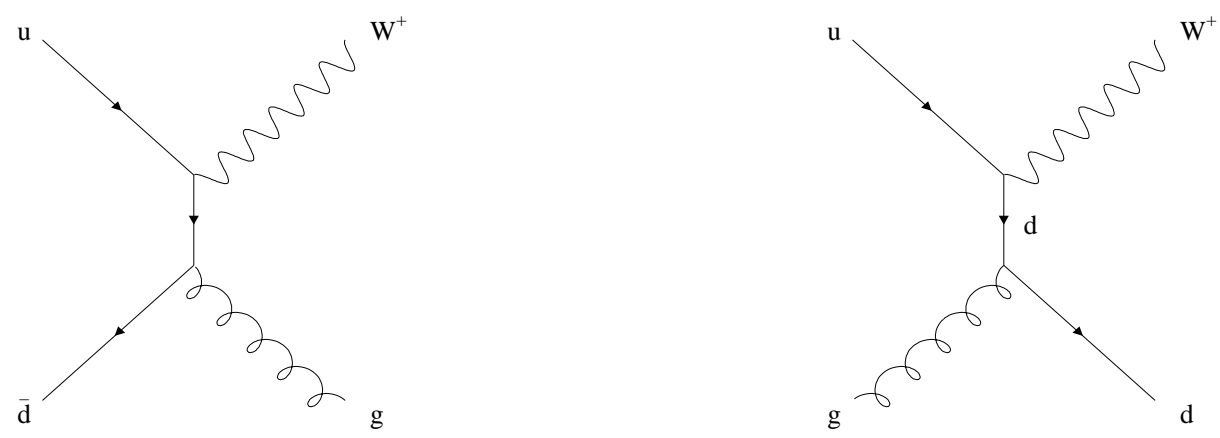

Figure 5.3: Higher order diagrams for $W$ production: (left) the inital state gluon radiation process and the (right) Compton process. Similar diagrams exist for $Z$ production.

are determined by Ladinsky and Yuan. They fit their hypothesis to the available Drell-Yan and $Z$ production data and obtain the values:

$$
g_{1}=0.11_{-0.03}^{+0.04} \mathrm{GeV}^{2} \quad g_{2}=0.58_{-0.2}^{+0.1} \mathrm{GeV}^{2} \quad g_{3}=-1.5_{-0.1}^{+0.1} \mathrm{GeV}^{-1}
$$

where $Q_{\circ}=1.6 \mathrm{GeV}$, and $b_{\max }=0.5 \mathrm{GeV}^{-1}$ are chosen. A study of the relative contributions of each of the three terms [81, chap. 3], shows that $g_{2}$ is the dominant parameter, and that the nominal values computed by Ladinsky and Yuan agree very well with the D $\varnothing$ data.

\subsection{3 $W$ and $Z$ Decays}

The decay of the $W$ or $Z$ boson is performed in its rest frame. The boson is decayed using the leading order diagrams shown in Figure 5.4. By assuming the leptons to be massless, the decay products are back-to-back. In addition, each decay 
product has an energy equal to $1 / 2$ the boson mass. The distribution of the azimuthal angle, $\phi$, of the leptons is uniform in the interval $[0,2 \pi)$.
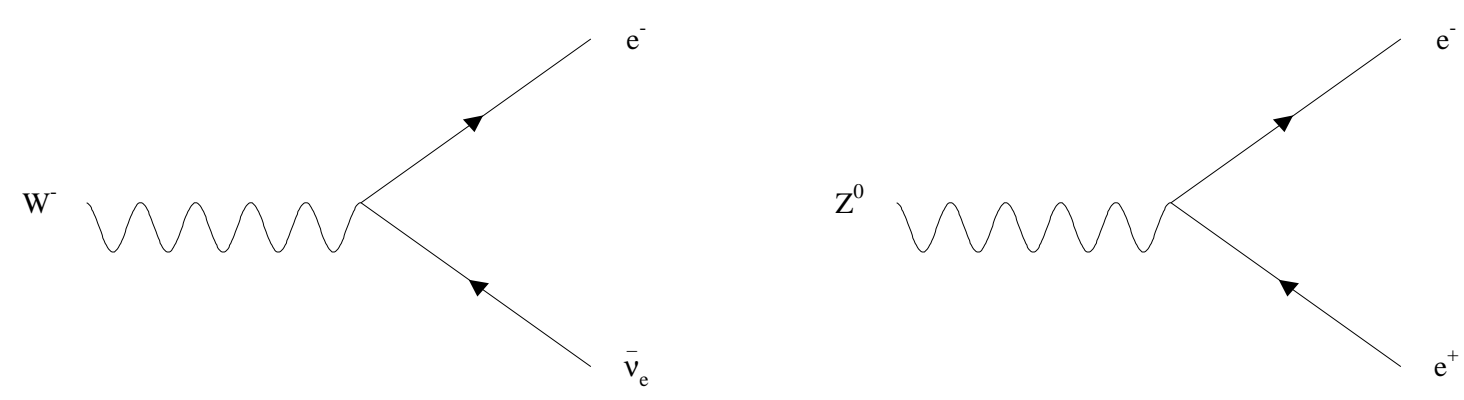

Figure 5.4: Leading order diagrams for $W \rightarrow e \nu$ and $Z \rightarrow e e$ decays.

For $W$ decays, the distribution of the polar angle, $\theta^{\star}$, is dependent on the $W^{\prime}$ s charge $Q$ and polarization $\lambda$ :

$$
\frac{d \sigma}{d \theta^{\star}} \propto\left(1-\lambda Q \cos \theta^{\star}\right)^{2}
$$

where $\theta^{\star}$ is the angle between the lepton and the $+z$-direction in the $W$ boson rest frame. For $Z$ decays, the $\theta^{\star}$ distribution is given by:

$$
\frac{d \sigma}{d \theta^{\star}} \propto 1+\cos ^{2} \theta^{\star}
$$

where $\theta^{\star}$ is again the angle between either lepton and $+z$-direction in the $Z$ boson rest frame. The absence of a term proportional to $\cos \theta^{\star}$ is due to the fact that $D \varnothing$ has no central magnetic field, and the charge of the decay lepton cannot be observed. At this point, the leptons are boosted into the lab frame using the four vector of the generated boson. 


\subsubsection{QED Radiative Decays}

The lowest order decay diagrams discussed in the previous section are corrected for the "internal bremsstrahlung" process, where a photon is radiated from the final state electron. This effect can be important since the radiated photon effectively lowers the measured energy of the electron. However, this effect is dependent on the energy of the photon and its separation from the electron. The calculation of Berends and Kleiss [86], performed at $\mathcal{O}\left(\alpha_{e m}\right)$, is used with a lower limit on the energy of the photon that may be produced. Approximately $31 \%$ of $W$ events, and $66 \%$ of $Z$ events, are allowed to radiate one photon with a minimum energy $\gamma_{\min }>50 \mathrm{MeV}[78$, p. 65]. In these radiative events, the electron, photon, and neutrino are then boosted into the lab frame. The energies of the photon and its associated electron are combined if their separation, $\mathcal{R}=\sqrt{\Delta \phi^{2}+\Delta \eta^{2}}$, is less than 0.3 ; otherwise, the photon is treated as a separate object.

\subsubsection{2 $W \rightarrow \tau \nu \rightarrow e \nu \nu$ Decays}

One of the options in the CMS Monte Carlo is the sequential decay of the $W$ to an electron via a $\tau$ intermediate state $(W \rightarrow \tau \nu \rightarrow e \nu \nu)$ for a specified fraction of the events. The process $W \rightarrow \tau \nu$ is generated in the same manner as $W \rightarrow e \nu$. The three body $\tau$ decay, $\tau \rightarrow e \nu \nu$, is performed in the rest frame of the $\tau$. The energy and angular correlations of the electron with respect to the $\tau$ polarization vector are preserved by selecting them from a two dimensional distribution obtained from $\tau \rightarrow e \nu \nu$ decays 
generated with the ISAJET Monte Carlo [87]. In the acceptance calculation of the $W$, this option is not utilized, since one is computing the acceptance of $W \rightarrow e \nu$ events exclusively. However, it becomes usefull in computing the irreducible $W \rightarrow \tau \nu$ background in $W \rightarrow e \nu$ events (see Section 7.1.2).

\subsection{Detector Response}

In order to accurately measure the acceptance of $W$ and $Z$ events, the response of the $\mathrm{D} \emptyset$ detector must be applied to the decay leptons. The relevant detector effects are the electromagnetic energy scale and resolutions, as well as the hadronic response and resolution.

\subsubsection{Electromagnetic Energy Scale}

The response model of the electromagnetic clusters is based on the test beam measurements, in which the true and measured energies are linearly related:

$$
E^{\text {true }}=\alpha E^{\text {meas }}+\beta
$$

where $\alpha$ is the EM energy scale and $\beta$ an energy offset. The energy offset was measured in low energy $J / \psi[88]$ and $\pi^{o}$ resonances [89], and the value is listed in Table 5.1. Since this correction is applied to the data, $\beta$ is set to 0 . in the CMS Monte Carlo.

The electromagnetic energy scale, $\alpha$, has already been discussed in Section 3.9. It is determined by a constrained fit of the dielectron invariant mass spectrum from 
a sample of clean $Z \rightarrow e e$ events to the $Z$ boson mass value measured very precisely by the four LEP experiments. An EM energy scale is measured for each cryostat, and the scale corrections, listed in Table 5.1, are applied to the data. Hence, a scale of 1.0 is applied to the events generated with CMS. The error in the CC EM scale is statistical, and is only limited by the number of available $Z \rightarrow e e$ events. The EC scale errors, however, are dominated by the systematic error. The latter is obtained by comparing the invariant mass distribution of CC-EC events with EC-EC events. A shift of $800 \mathrm{MeV}$ in the peak of the distribution is observed, which leads to a rather large error on the value of the EC scale. This shift is probably caused by a miscalibration of the EC calorimeter module and/or the FDC trackers.

Table 5.1: EM energy calibration constants applied to the data and in the CMS Monte Carlo.

\begin{tabular}{|c|c|c|c|c|}
\hline \multicolumn{2}{|c|}{$\overline{\bar{P}}$ Parameter } & $\overline{\mathrm{CC}}$ & $\overline{\text { EC South }}$ & $\overline{\text { EC North }}$ \\
\hline \multirow{2}{*}{ Data } & $\alpha$ & 1.072 & 1.112 & 1.025 \\
\hline & $\beta$ & & $-0.158 \mathrm{GeV}$ & \\
\hline \multirow{2}{*}{ CMS } & $\alpha$ & $1.000 \pm 0.001$ & $1.000 \pm 0.013$ & $1.000 \pm 0.012$ \\
\hline & $\bar{\beta}$ & & $0.00_{-0.21}^{+0.03} \mathrm{GeV}$ & \\
\hline
\end{tabular}

\subsubsection{Electromagnetic Energy Resolutions}

The electromagnetic energy resolution is parametrized as follows:

$$
\Delta E=\mathcal{C} \cdot E \oplus \mathcal{S} \cdot \sqrt{E_{T}}
$$

where $\mathcal{C}$ is the constant term, and $\mathcal{S}$ is the sampling term. The square root of the transverse energy, $\sqrt{E_{T}}$, is used in conjunction with the sampling term for the central 
calorimeter, while the square root of the energy, $\sqrt{E}$, for the forward calorimeter. The values of the resolution parameters are listed in Table 5.2. The values of the sampling term, $\mathcal{S}$, are obtained from test beam studies [90], and are assumed to be exact.

The constant term, $\mathcal{C}$, is determined from the width of the invariant mass distribution, $\sigma\left(M_{e e}\right)$, in $Z \rightarrow e e$ events as shown in Figure 5.5a. By varying the constant term in the Monte Carlo, the best fit to the invariant mass distribution in the data is obtained (see Figure 5.5b).

Table 5.2: EM energy resolution parameters used in the CMS Monte Carlo.

\begin{tabular}{ccc}
\hline \hline Parameter & Central Calorimeter & Forward calorimeter \\
\hline $\mathcal{C}$ & $0.014 \pm 0.002$ & $0.00_{-0.00}^{+0.01}$ \\
$\mathcal{S}$ & $0.135 \mathrm{GeV}^{1 / 2}$ & $0.157 \mathrm{GeV}^{1 / 2}$ \\
\hline \hline
\end{tabular}
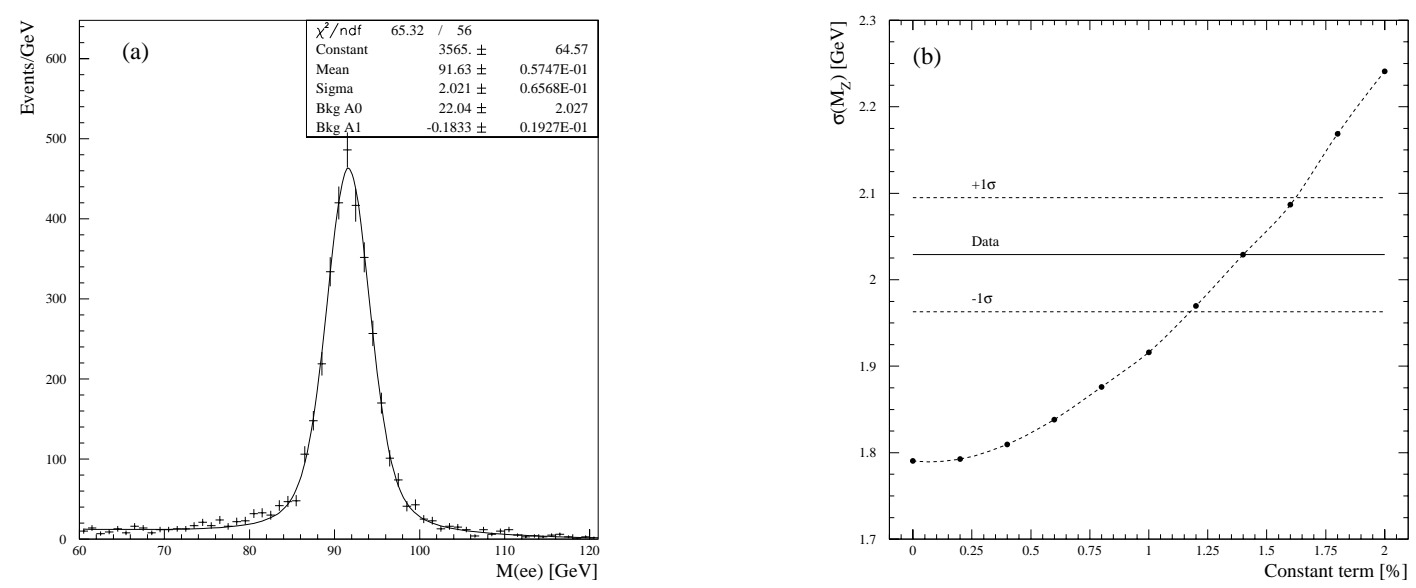

Figure 5.5: (a) Simple Breit-Wigner convoluted with a gaussian resolution fit to the central $Z \rightarrow e e$ invariant mass spectrum. (b) Predicted $\sigma\left(M_{e e}\right)$ vs $\mathcal{C}$ for data (line) and Monte Carlo (points) for central electrons. 


\subsubsection{Hadronic Response and Resolutions}

A $W$ or $Z$ event is composed of two parts: the hard scattering which produces the vector boson, and the hadronization of the spectator quarks. The hard scattering produces the vector boson in question, along with a recoil jet-like energy flow in the opposite direction to the initial transverse momentum of the vector boson. The spectator quarks produce a softer energy flow, which is azimuthally symmetric, called the underlying event. The model employed in the CMS Monte Carlo to simulate the hadronic response incorporates these two components: the recoil and the underlying event.

The resolution of the recoil that balances the initial $p_{T}$ of the boson is assumed to be modeled according to the single jet resolution:

$$
\Delta p_{T}=\mathcal{C}_{H} \cdot p_{T} \oplus \mathcal{S}_{H} \cdot \sqrt{p_{T}}
$$

where the values $\mathcal{C}_{H}=4 \%$ and $\mathcal{S}_{H}=80 \%$ are taken from the DØ QCD working group [91] and are assumed to be exact.

The contribution from the underlying event is modeled using minimum bias data. A library of minimum bias events is constructed from events that were collected throughout the data taking period. Since these events do not contain any high- $p_{T}$ neutrinos, their $\mathbb{E}_{T}$ distribution reflects the resolution due to noise, pile-up, and other activity which mimics the behavior of the spectator quarks. The $\mathbb{E}_{T}$ from a randomly chosen minimum bias event is added to the Monte Carlo. Its direction is chosen at a 
random direction in azimuth, while its magnitude is scaled by a factor, $\alpha_{M B}$, which allows for the tuning of the hadronic resolution.

\subsubsection{Hadronic Scale}

The energy scale of the hadronic recoil of the vector boson is referred to as the hadronic response, or the hadronic energy scale $\left(\alpha_{H}\right)$. This hadronic energy scale is measured relative to the electromagnetic energy scale, by comparing the $p_{T}(Z)$ measured from the electron pair, to the $p_{T}(Z)$ measured from the hadronic system. The hadronic response is then defined as:

$$
\vec{p}_{T}^{r e c}=\alpha_{H} \vec{p}_{T}^{e e}
$$

In order to perform this comparison, it is useful to define a coordinate system in the transverse plane which depends on the electron directions, and not their momenta. The $\eta$-axis ${ }^{2}$ is chosen as the bisector of the electron directions in the transverse plane, while the $\xi$-axis is perpendicular to it. An illustration of this definition is provided in Figure 5.6.

The projection of the recoil onto the $\eta$-axis is minimally sensitive to the electron energy resolution. Hence, the hadronic scale is constrained by measuring the dependence of $\left(\vec{p}_{T}^{r e c}+\vec{p}_{T}^{e e}\right) \cdot \hat{\eta}$ on $\vec{p}_{T}^{e e} \cdot \hat{\eta}$. Since the true momentum vectors of the dielectron and recoil systems are equal and opposite by momentum conservation, the vectorial sum $\left(\vec{p}_{T}^{r e c}+\vec{p}_{T}^{e e}\right)$ is zero on average, if the EM and hadronic responses are

\footnotetext{
${ }^{2}$ This $\eta$ is completely unrelated to the pseudorapidity.
} 


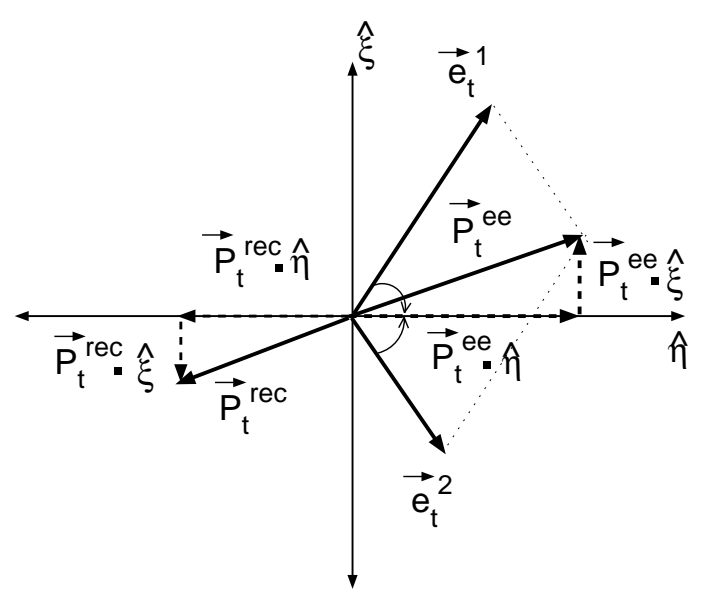

Figure 5.6: Definition of the $\eta-\xi$ coordinate system in a $Z$ boson event. The $\eta$ axis is the bisector of the electrons in the transverse plane; the $\xi$ axis is perpendicular to $\eta[78$, p. 46].

equivalent. For the reasons pointed out in Section 2.5, the hadronic scale is usually lower than the EM scale. Hence, a relative scale $\alpha_{H}$ would cause a slope of $\sim\left(1-\alpha_{H}\right)$ in the plot of the $\eta$-balance: $\left(\vec{p}_{T}^{r e c}+\vec{p}_{T}^{e e}\right) \cdot \hat{\eta}$ vs $\vec{p}_{T}^{e e} \cdot \hat{\eta}$. This $\eta$-balance plot is shown in Figure 5.7a. The CMS Monte Carlo is used to derive fit slope values of the $\eta$-balance as a function of $\alpha_{H}$, as is shown in Figure $5.7 \mathrm{~b}$. The hadronic response is found to be $\alpha_{H}=0.753 \pm 0.012$. The error on $\alpha_{H}$ is assigned a 0.021 systematic error due to the uncertainties in the EC electromagnetic scale. Thus, the hadronic response is $\alpha_{H}=0.753 \pm 0.024$. The data is not corrected for this scale factor, hence $\alpha_{H}$ is applied to CMS.

\subsubsection{Hadronic resolution}

To measure the hadronic resolution parameters, the CMS Monte Carlo is used to determine the resolution of the $\eta$-balance as a function of $\alpha_{M B}$, the number of minimum bias events added to the generated vector boson. This $\eta$-balance width is 

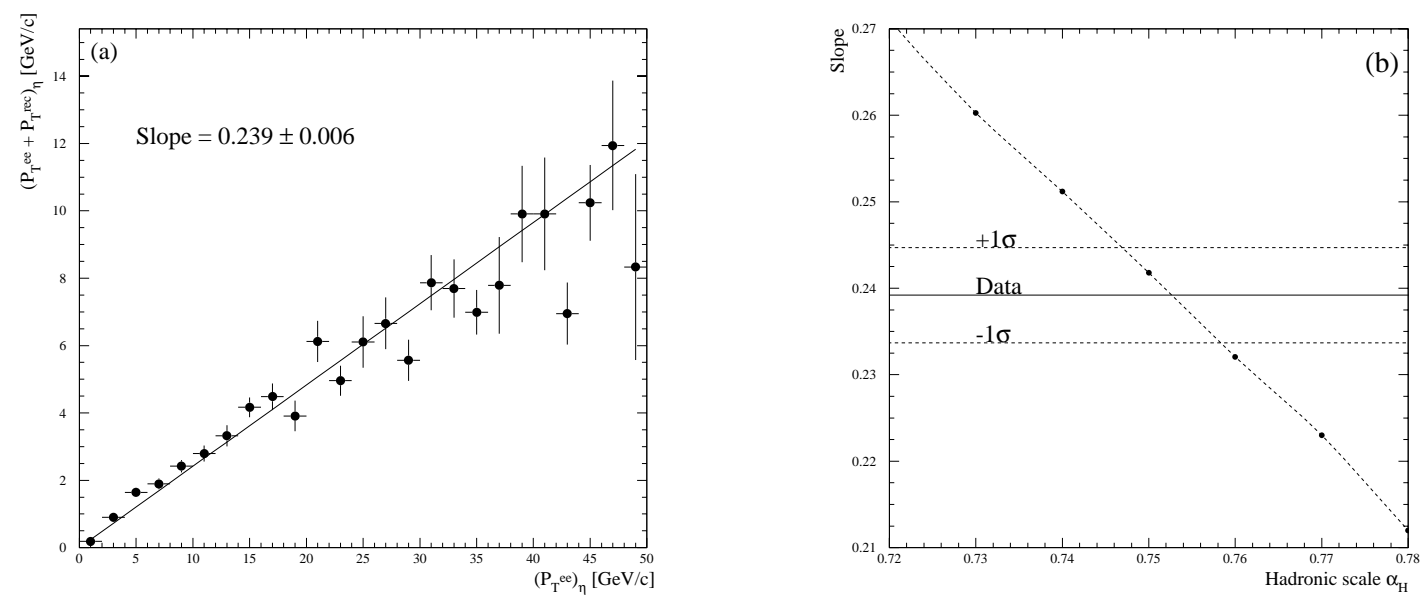

Figure 5.7: (a) The fit to the $\eta$-balance in $Z \rightarrow e e$ events. (b) Predicted $\eta$-balance vs $\alpha_{H}$ values (points) as obtained in CMS, and the $\eta$-balance for the data (line).
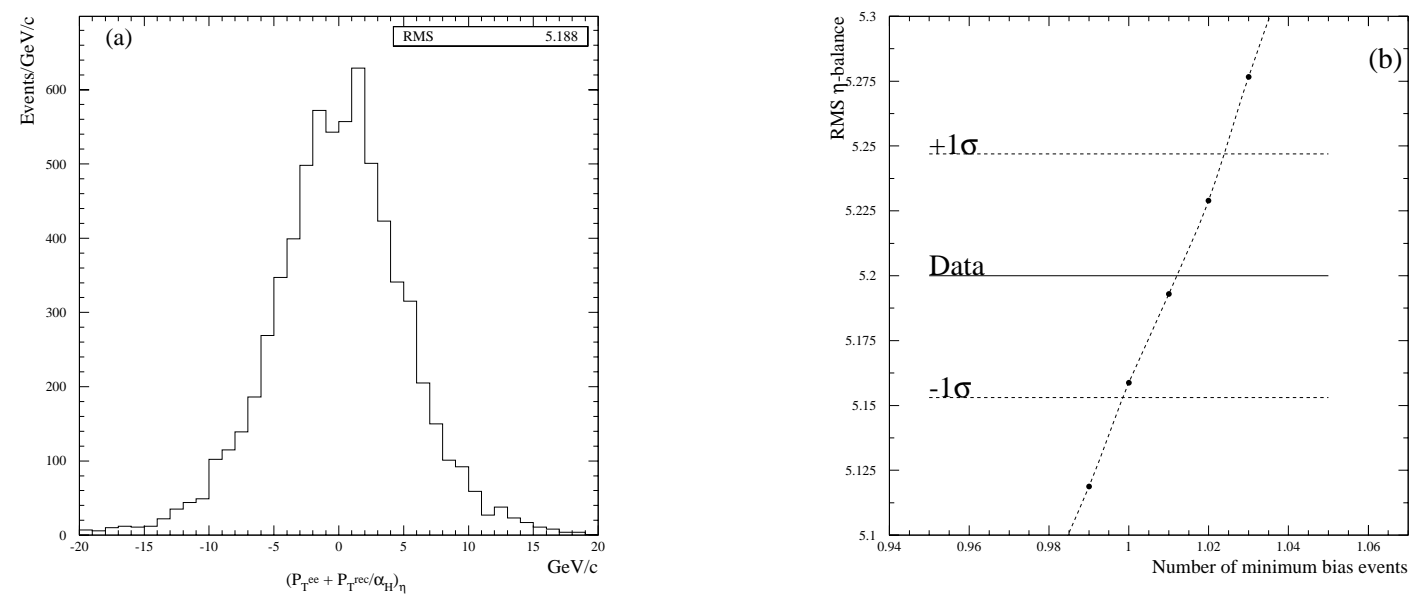

Figure 5.8: (a) The $\eta$-balance width (RMS of the $\alpha_{H}$ corrected $\eta$-balance) in $Z \rightarrow e e$ events. (b) Predicted $\eta$-balance width vs $\alpha_{M B}$ for Monte Carlo (points) and the data (line). 
computed with the hadronic recoil vector that is corrected for the hadronic response: the $\eta$-balance is equal to $\left(\vec{p}_{T}^{e e}+\vec{p}_{T}^{r e c} / \alpha_{H}\right) \cdot \hat{\eta}$. The plot of the $\eta$-balance width is shown in Figure 5.8a, where a value of $\alpha_{H}=0.753$ is used.

The CMS Monte Carlo is used to derive $\eta$-balance width values as a function of $\alpha_{M B}$, as is shown in Figure 5.8b. The number of minimum bias events is found to be $\alpha_{M B}=1.01 \pm 0.02$, which is consistent with one. A summary of the measured hadronic parameters and their errors used in the CMS Monte Carlo is presented in Table 5.3.

Table 5.3: Hadronic scale factors and resolutions used in the CMS Monte Carlo.

\begin{tabular}{cccc}
\hline \hline$\alpha_{H}$ & $\mathcal{C}_{H}$ & $\mathcal{S}_{H}$ & $\alpha_{M B}$ \\
\hline $0.753 \pm 0.012$ & 0.04 & 0.50 & $1.01 \pm 0.02$ \\
\hline \hline
\end{tabular}

\subsubsection{Underlying Event Corrections}

The underlying event contributes energy symmetrically in the detector. Due to the electron and transverse energy reconstruction algorithms, the CMS Monte Carlo electron and recoil vectors need to be corrected for two effects. Firstly, the electron energy needs to be increased by the contribution from the underlying event flowing into the detector area occupied by the electron cluster; this correction is denoted by $E_{u e}$. Secondly, the recoil vector must be decreased for the energy lost under the electron; this correction is denoted by $\Delta U_{\|}$. Even though these two corrections should 
be equal and opposite, the zero suppression during the calorimeter cell readout (see Section 2.5.5) causes them to be different $\left(E_{u e}<\Delta U_{\|}\right)$.

The correction to the recoil vector is obtained from $W$ boson events by rotating azimuthally $90^{\circ}$ away from the electron cluster and summing the energy in an area equal to that occupied by the electron cluster. The applied correction to the recoil vector is dependent on the instantaneous luminosity, and on the parallel component of the underlying event with respect to the electron direction. The mean value of this correction for the entire data sample is $460 \pm 25 \mathrm{MeV}$.

The correction to the electron vector is determined from ISAJET electrons generated with the same $\eta$ and $E_{T}$ distribution as electrons from $W$ boson events. These electrons are used to prepare two data sets. The first is obtained by subjecting them to DØGEANT, and performing the reconstruction using DØRECO. The second is obtained by overlapping them with non-zero suppressed minimum bias events, then passing them through DØGEANT, and reconstructing them with DØRECO. The energy flow under the electron is computed on an event by event basis, by taking the difference in the reconstructed energy of the two data samples. The mean value of the correction is found to be $215 \pm 25 \mathrm{MeV}$.

In the CMS Monte Carlo, the corrections $E_{u e}$ and $\Delta U_{\|}$are modeled according to the distributions determined in the data. These corrections act as effective noise terms in the resolution functions of Equation 5.12 and Equation 5.13. 


\section{$5.3 \quad W$ and $Z$ Acceptances}

For the central value of the acceptance calculation, the MRSAp structure function [92] is used, along with the following values for the vector boson masses and natural widths:

$$
\begin{gathered}
M_{W}=80.45 \mathrm{GeV} / \mathrm{c}^{2}, \quad,{ }_{W}=2.07 \mathrm{GeV} \\
M_{Z}=91.188 \mathrm{GeV} / \mathrm{c}^{2}, \quad,{ }_{Z}=2.496 \mathrm{GeV} .
\end{gathered}
$$

The $W$ mass value corresponds to the latest world average [93], while the $W$ natural width to the current world average which incorporates the Run Ia D $\varnothing$ and CDF measurements [18]. The parameters of the $Z$ boson were obtained from the Particle Data Group [18], are are based on the very precise measurements of the LEP experiments.

To calculate the acceptance, 25 million $W \rightarrow e \nu$ events, $N_{W}($ gen $)$, and 25 million $Z \rightarrow e e$ events, $N_{Z}($ gen $)$, are generated with the CMS Monte Carlo. The $W$ acceptance is calculated from the number of events, $N_{W}$ (pass), which contain the following:

- Electron with $E_{T}>25 \mathrm{GeV}$, where the electron satisfies the fiducial cuts as defined in Section 4.1;

- $\not_{T}>25 \mathrm{GeV}$.

The $W$ acceptance is computed as:

$$
\mathcal{A}_{W}=\frac{N_{W}(\text { pass })}{N_{W}(\text { gen })}
$$

The $Z$ acceptance is calculated from the number of events, $N_{Z}$ (pass), which contain the following: 
- 2 electrons with $E_{T}>25 \mathrm{GeV}$, where the electrons satisfy the fiducial cuts as defined in Section 4.1;

- $75<M_{e e}<105 \mathrm{GeV} / \mathrm{c}^{2}$.

The $Z$ acceptance is computed as:

$$
\mathcal{A}_{Z}=\frac{N_{Z}(\text { pass })}{N_{Z}(\text { gen })} .
$$

The measured acceptances are summarized in Table 5.4. The statistical errors are negligible $(\sim 0.02 \%)$, while the systematic errors are obtained by varying the input parameters to the CMS Monte Carlo (this will be further explained in the next section).

Table 5.4: Absolute and relative acceptances of $Z$ and $W$ boson events (quoted errors are statistical $\oplus$ systematic).

\begin{tabular}{cccc}
\hline \hline \multicolumn{2}{c}{ Acceptance } & Absolute & Relative \\
\hline \multirow{4}{*}{$\mathcal{A}_{Z}$} & CC-CC & $0.1857 \pm 0.0015$ & 0.4959 \\
& CC-EC & $0.1511 \pm 0.0013$ & 0.4036 \\
& EC-EC & $0.0376 \pm 0.0003$ & 0.1004 \\
\cline { 2 - 4 } & Total & $0.3744 \pm 0.0031$ & 1.0000 \\
\hline \hline \multirow{2}{*}{$\mathcal{A}_{W}$} & CC & $0.3284 \pm 0.0026$ & 0.6967 \\
& EC & $0.1430 \pm 0.0011$ & 0.3033 \\
\cline { 2 - 4 } & Total & $0.4714 \pm 0.0038$ & 1.0000 \\
\hline \hline
\end{tabular}

\subsubsection{Systematic Errors}

Any uncertainties in the event generation or detector simulation in the CMS Monte Carlo will cause an error in the measured acceptances. Some uncertainties are 
due to the theoretical production model, such as the vector boson $p_{T}$ spectrum, the the choice of structure functions, the choice of photon-electron separation criteria in radiative events, and the choice of vector boson mass and natural width. Other uncertainties are due to the parametrization of the detector response. The errors on the acceptances are summarized in Table 5.5, where the quoted errors on the $W$ and $Z$ acceptances are given as a percentage of the acceptance. In addition, the error on the acceptance ratio, $\delta\left(\mathcal{A}_{Z} / \mathcal{A}_{W}\right)$, is also given in terms of the acceptance ratio, $\mathcal{A}_{Z} / \mathcal{A}_{W}$

Table 5.5: Systematic errors on the acceptance of $W$ and $Z$ boson events. The total errors are obtained by summing in quadrature all the individual errors.

\begin{tabular}{lccc}
\hline \hline Error source & $\frac{\delta \mathcal{A}_{W}}{\mathcal{A}_{W}}[\%]$ & $\frac{\delta \mathcal{A}_{Z}}{\mathcal{A}_{Z}}[\%]$ & $\delta\left(\frac{\mathcal{A}_{Z}}{\mathcal{A}_{W}}\right) /\left(\frac{\mathcal{A}_{Z}}{\mathcal{A}_{W}}\right)[\%]$ \\
\hline$p_{T}$ spectrum & 0.29 & 0.14 & 0.24 \\
Structure functions & 0.40 & 0.60 & 0.30 \\
QED corrections & 0.16 & 0.31 & 0.15 \\
$\delta M_{W}$ & 0.17 & - & 0.17 \\
$\delta, W$ & 0.08 & - & 0.08 \\
EM energy scale & 0.55 & 0.37 & 0.30 \\
EM energy resolution & 0.04 & 0.08 & 0.06 \\
Hadronic response & 0.15 & - & 0.15 \\
Hadronic resolution & 0.07 & - & 0.07 \\
Angular resolution & 0.03 & 0.08 & 0.05 \\
\hline Total & $0.80 \%$ & $0.83 \%$ & $0.62 \%$ \\
\hline \hline
\end{tabular}

The largest error in the $W$ and $Z p_{T}$ spectrum is in the soft, non-perturbative regime, where the resummation calculation is most important. Since the $g_{2}$ parameter in the Ladinsky-Yuan model (see Section 5.1.2) is the dominant parameter, the error due to the $p_{T}$ spectrum on the acceptances is estimated by varying it within its 
measured range of values. Changing $g_{2}$ by $\pm 0.2 \mathrm{GeV}^{2}$ leads to a $0.29 \%$ change in the $W$ acceptance and a $0.14 \%$ change in the $Z$ acceptance.

The systematic error due to the choice of structure functions is calculated by using the MRSAp structure functions with varying values of the strong coupling constant $\alpha_{S}$ : MRSAp-150, MRSAp-201,MRSAp-266, and MRSAp-344 [94]. Variations in $\alpha_{S}$ causes a variation in the amount of gluons produced, which affects the kinematics of the generated vector boson, and its decay leptons. The largest difference is used to estimate the error of $0.40 \%$ for the $W$ acceptance and $0.60 \%$ for the $Z$ acceptance.

The chosen value of the $W$ mass is the current world average value, and has an error of $125 \mathrm{MeV}$; varying $M_{W}$ by this amount leads to a $0.17 \%$ change in the $W$ acceptance. Similarly, the chosen value of the $W$ natural width is the current world average value, and has an error of $60 \mathrm{MeV}$. Varying this width by $240 \mathrm{MeV}$ $( \pm 4 \sigma)$, leads to a change in the acceptance of $0.08 \%$. Since the values of the $Z$ boson mass and width are known quite accurately, their effect on the acceptance are safely neglected.

The error due to the QED radiative corrections is estimated by varying the cone size used in deciding whether the radiated photon's energy should be merged with its associated electron. The cone variation of $\delta \mathcal{R}= \pm 0.1$ leads to a $0.16 \%$ change in the $W$ acceptance, and a $0.31 \%$ change in the $Z$ acceptance.

The EM energy scale and resolution are varied within their measured errors. The error due to the EM energy scale, $\alpha$, is $0.55 \%$ in the $W$, and $0.37 \%$ in the $Z$ 
acceptance; the errors due to variations in the constant term $\mathcal{C}$ are $0.04 \%$ and $0.08 \%$ for the $W$ and $Z$ acceptances respectively.. The hadronic scale and resolution are also varied within their measured errors. The change in the $W$ acceptance due to variations in $\alpha_{H}$ is $0.15 \%$, while the change due to variations in $\alpha_{M B}$ is $0.07 \%$. The errors due to the underlying event corrections $E_{u e}$ and $\Delta U_{\|}$, as well as the energy offset $\beta$, are negligible.

Finally, the largest uncertainty in the determination of the electron angles is due to the uncertainty on the bias in the measurement of the track center-of-gravity. Hence, the estimate of the acceptance error due to the angular resolution is done by varying the $z$-position ( $r$-position) of the central (forward) track's center-of-gravity within its measured error. This leads to a $0.03 \%$ and $0.08 \%$ change on the $W$ and $Z$ acceptances, respectively. 


\section{Chapter 6}

\section{Detection Efficiencies}

The trigger and offline selection cuts used in identifying genuine electrons and reducing the background cause a fraction of the real signal to be lost. For example, shower fluctuations could cause an electron to be rejected by the isolation or shower shape requirement. This chapter concerns the measurement of the efficiencies of these cuts, where the efficiency reflects the frequency at which events are lost due to the cuts themselves.

\subsection{Methods}

The accurate measurement of the efficiency of a selection cut requires one to have a clean sample of unbiased electrons. This sample, called the diagnostic sample, should in principle be selected with a minimum of cuts, thus avoiding the introduction of any bias. These cuts must also be uncorrelated with the one that is under study. One might be tempted to consider a beam of pure electrons delivered to the detector. 
This was indeed done in test beam measurements where the initial attempts at defining the electron selection criteria were developed. However, it is not possible, much less worthwhile, to deliver such a beam to the actual DØ detector. The alternative is then to rely on the collider data to provide the required diagnostic sample from which the selection criteria can be measured. Samples of electrons from $W$ and $Z$ events are perfect candidates for such diagnostic samples, since the electrons then have all the characteristics (e.g. the underlying event or multiple interactions effects) which might affect the efficiencies that need to be measured.

Despite its large size, efficiency measurements from a sample of $W$ electrons is quite difficult since the reconstruction of the $W$ invariant mass is impossible with the presence of a neutrino. Without a characteristic Breit-Wigner from which signal and background is separated, one is reduced to using electron selection criteria just to define the diagnostic sample, thus introducing bias and possible correlation effects.

$Z \rightarrow e e$ events are much better suited for the task at hand: by requiring the reconstructed dielectron invariant mass to be close to $M_{Z}$, and by imposing the tight electron identification criteria on one of the electrons, one obtains a clean sample of unbiased, or probe, electrons which contain little background [95][96]. The efficiency of an offline cut is measured by looking at the change in the number of probe electrons after they are subjected to the cut. Hence, the definition of the efficiency of a cut " $a$ " relative to a (looser) cut " $b$ " is given by:

$$
\varepsilon_{a b}=\frac{N_{a b}}{N_{b}}
$$


where $N_{a b}$ and $N_{b}$ denote the background subtracted number of probe electrons which pass cuts " $a$ " and " $b$ " together, and cut " $b$ " alone, respectively. Of course, the precision of the measurement is statistically limited by the number of available $Z \rightarrow e e$ events. In addition, any uncertainty in the determination of the amount of background will give rise to a systematic uncertainty in the efficiency measurement.

\subsubsection{Diagnostic Data Sample}

The sample of diagnostic electrons is obtained from a $Z \rightarrow e e$ sample which passed the EM2_EIS_ESC Level-2 filter. This filter basically looked for two EM clusters where one cluster was isolated and was required to have transverse energy $E_{T}^{\mathrm{L} 2}>20$ $\mathrm{GeV}$, while the other was only required to possess transverse energy $E_{T}^{\mathrm{L} 2}>16 \mathrm{GeV}$. Since a Level-2 transverse energy cut of $16 \mathrm{GeV}$ is $100 \%$ efficient for electrons with offline transverse energy $E_{T}>25 \mathrm{GeV}$, this loosely triggered electron becomes the probe electron from which the efficiency of the offline selection cuts will be measured.

The selection procedure starts with events passing the EM2_EIS_ESC filter and which contain two electrons in the standard fiducial region of the detector. Tagging cuts are applied on one of the electrons. These cuts include a Level-2 trigger requirement as well as offline selection criteria. The tagging cuts are as follows:

- EM cluster passes L2 eis20 requirement ${ }^{1}$;

\footnotetext{
${ }^{1}$ The Level-2 shower shape cut is denoted by ele; the Level-2 isolation cut is denoted by $i s o$; requiring both is denoted by eis, while requiring neither is denoted by esc. In addition, the following convention is adopted: the value of the Level- $2 E_{T}$ cut is appended to the Level-2 terms, hence eis 20 denotes an electron with $E_{T}^{\mathrm{L} 2}>20 \mathrm{GeV}$ which passes Level-2 shower shape and isolation cuts.
} 
- H-matrix $\chi_{h m}^{2}<100$;

- EM fraction $f_{e m}>0.95$;

- Isolation fraction $f_{i s o}<0.15$

- A track exists with $S_{t r k}<5(10)$ in the CC(EC).

If an electron passes the tagging cuts, then it is used to determine the $z$-coordinate of the event, from which the transverse energy of the electrons are computed. Both electrons are required to pass a transverse energy $E_{T}>25 \mathrm{GeV}$ cut. In addition, the invariant mass of the two electrons, $M_{e e}$, is required to be very close to the true $Z$ boson mass $\left(M_{Z}=91.2 \mathrm{GeV} / \mathrm{c}^{2}\right)$. It is important to note that the tagging procedure is applied for each EM cluster in the event: in cases where both electrons pass the tagging cuts, the event is used twice in the efficiency studies.

\subsubsection{Background Determination}

In order to quantify the uncertainty due to the background subtraction mechanism, four different methods are employed to estimate it:

A. A sideband averaging technique is used. Lower and upper sideband regions are defined outside the signal region of $86<M_{e e}<96 \mathrm{GeV} / \mathrm{c}^{2}$ in order to estimate the amount of background. The lower sideband encompasses the region $60<M_{e e}<70 \mathrm{GeV} / \mathrm{c}^{2}$, while the upper sideband the region $110<M_{e e}<120$ $\mathrm{GeV} / \mathrm{c}^{2}$. These regions are chosen to be symmetric about the signal region, and 
cover the same range in invariant mass. The number of background events is then taken to be the average of the two sideband regions;

B. Method $A$ is repeated for a signal region $81<M_{e e}<101 \mathrm{GeV} / \mathrm{c}^{2}$. With this signal region, the number of background events is taken to be the sum of the two sideband regions (Figure 6.1a);

$C$. The dielectron mass spectrum is fit using a Breit-Wigner convoluted with a Gaussian (to account for the resolution in the measurement) and a linear background in the region $70<M_{e e}<110 \mathrm{GeV} / \mathrm{c}^{2}$. The linear fit parameters are then used to estimate the number of background events which must be subtracted out. The signal window is $86<M_{e e}<96 \mathrm{GeV} / \mathrm{c}^{2}$;

D. Method $C$ is repeated for a signal window $81<M_{e e}<101 \mathrm{GeV} / \mathrm{c}^{2}$ (Figure 6.1b).

A summary of the four methods employed is shown in Table 6.1.
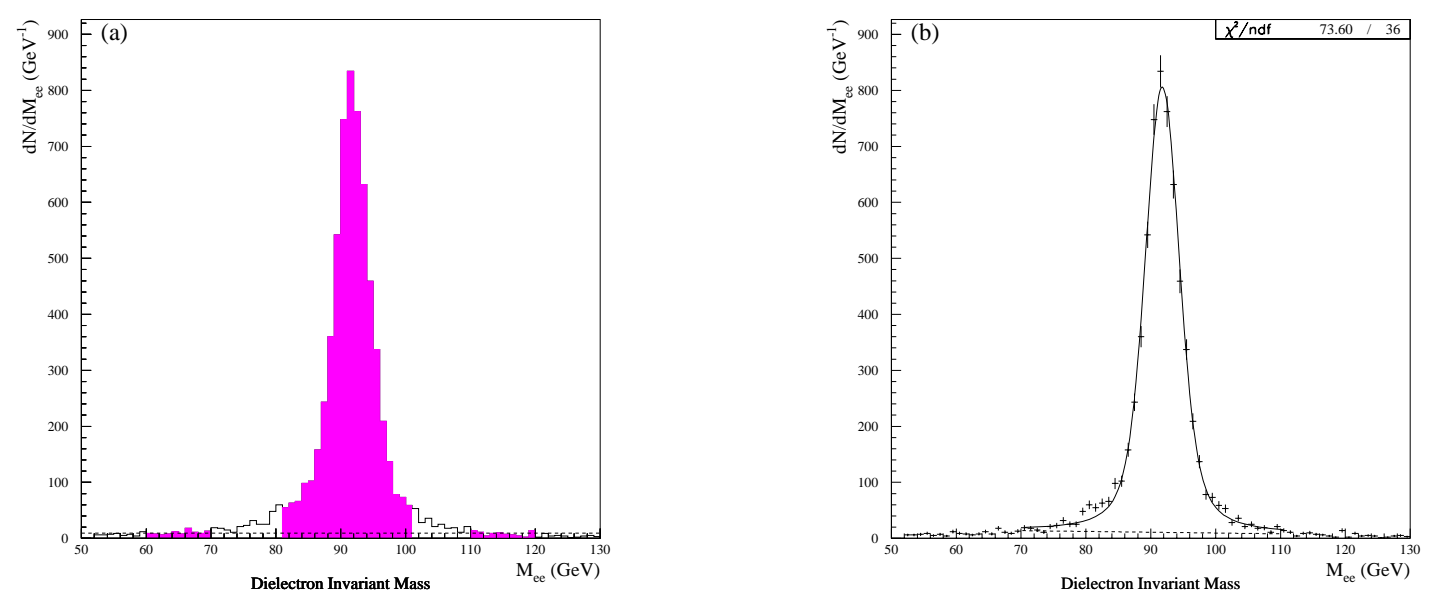

Figure 6.1: Illustration of the background subtraction mechanism for the (a) sideband technique, and (b) fit technique. The dashed line shows the estimated level of background. 
Table 6.1: Summary of the background subtraction methods used in estimating electron selection efficiencies.

\begin{tabular}{ccl}
\hline \hline Method & Background Subtraction & Signal Peak Range \\
\hline$A$ & Sideband & $86<M_{e e}<96 \mathrm{GeV} / \mathrm{c}^{2}$ \\
$B$ & Sideband & $81<M_{e e}<101 \mathrm{GeV} / \mathrm{c}^{2}$ \\
$C$ & $\mathrm{BW} \otimes \mathrm{G}$ fit & $86<M_{e e}<96 \mathrm{GeV} / \mathrm{c}^{2}$ \\
$D$ & $\mathrm{BW} \otimes \mathrm{G}$ fit & $81<M_{e e}<101 \mathrm{GeV} / \mathrm{c}^{2}$ \\
\hline \hline
\end{tabular}

\subsection{Single Electron Efficiencies}

Five different cuts are imposed on the probe electrons, creating five different electron classes:

1. Probe electron: an electron which passes the Level-2 esc16 required in the trigger. All other cuts are measured relative to this baseline cut;

2. Trigger electron: a probe electron which passes the Level-2 eis20 requirement;

3. Track electron: a trigger electron which passes the tracking requirements, namely a matching track with $S_{t r k}<5(10)$ in the CC(EC);

4. Loose electron: a trigger electron which passes the calorimetric identification criteria, namely $f_{e m}>0.95, \chi_{h m}^{2}<100$, and $f_{\text {iso }}<0.15$;

5. Tight electron: a loose and track electron.

The number of signal (i.e. background subtracted) electrons which are contained in each category are summarized in Table 6.2. They form the basis for three different 
relative efficiencies which are measured:

$$
\begin{array}{cc}
\text { Level-2 trigger efficiency } & \varepsilon_{\mathrm{L} 2} \equiv \frac{\# \text { Trigger electrons }}{\# \text { Probe electrons }}, \\
\text { Calorimeter ID efficiency } & \varepsilon_{\text {cal }} \equiv \frac{\# \text { Loose electrons }}{\text { \# Trigger electrons }}, \\
\text { and Tracking efficiency } & \varepsilon_{t r k} \equiv \frac{\# \text { Tight electrons }}{\# \text { Loose electrons }} .
\end{array}
$$

For example, the tracking efficiency for central electrons using method $A$ is:

$$
\varepsilon_{t r k}=\frac{4984}{6339}=0.7862 \pm 0.0051
$$

where the statistical error is binomial:

$$
\varepsilon=\frac{\mathrm{N}}{\mathrm{M}} \quad \Longrightarrow \quad \delta \varepsilon_{\text {stat }}=\sqrt{\frac{\varepsilon \cdot(1-\varepsilon)}{\mathrm{M}}}
$$

The results of the relative efficiency calculations are listed in Table 6.3 and Table 6.4 for central and forward electrons, respectively.

\subsection{Trigger Efficiency}

The single electron efficiencies computed in the previous section took into account the electron Level-2 terms ( $E_{T}$ and isolation). However, two important factors remain to be measured: the efficiency of the Level- $\varnothing$ system for detecting $W$ and $Z$ bosons, and the efficiency of the Level-2 trigger $\mathbb{E}_{T}$ requirement. 
Table 6.2: Number of (background subtracted) central (CC) and forward (EC) electrons contained in the $Z \rightarrow e e$ signal window for various offline cuts and background subtraction methods.

\begin{tabular}{lcccccccc}
\hline \hline \multirow{2}{*}{ Electron type } & \multicolumn{4}{c}{$\mathrm{CC}$} \\
\cline { 2 - 9 } & $N_{A}$ & $N_{B}$ & $N_{C}$ & $N_{D}$ & $N_{A}$ & $N_{B}$ & $N_{C}$ & $N_{D}$ \\
\hline Probe & 6912 & 8207 & 6860 & 8102 & 2884 & 3504 & 2903 & 3545 \\
Trigger & 6811 & 8059 & 6776 & 7989 & 2864 & 3497 & 2883 & 3536 \\
Track & 5292 & 6210 & 5277 & 6178 & 2093 & 2540 & 2119 & 2593 \\
Loose & 6339 & 7466 & 6299 & 7376 & 2485 & 3023 & 2510 & 3074 \\
Tight & 4984 & 5832 & 4966 & 5797 & 1819 & 2202 & 1844 & 2253 \\
\hline \hline
\end{tabular}

Table 6.3: Central electron relative efficiencies (quoted errors are statistical).

\begin{tabular}{cccc}
\hline \hline & \multicolumn{3}{c}{ CC electron efficiencies } \\
\hline Method & $\varepsilon_{\mathrm{L} 2}$ & $\varepsilon_{\text {cal }}$ & $\varepsilon_{\text {trk }}$ \\
\hline$A$ & $0.9853 \pm 0.0014$ & $0.9170 \pm 0.0033$ & $0.7862 \pm 0.0052$ \\
$B$ & $0.9820 \pm 0.0015$ & $0.9097 \pm 0.0032$ & $0.7811 \pm 0.0048$ \\
$C$ & $0.9878 \pm 0.0013$ & $0.9182 \pm 0.0033$ & $0.7884 \pm 0.0052$ \\
$D$ & $0.9861 \pm 0.0013$ & $0.9116 \pm 0.0032$ & $0.7849 \pm 0.0048$ \\
\hline \hline
\end{tabular}

Table 6.4: Forward electron relative efficiencies (quoted errors are statistical).

\begin{tabular}{cccc}
\hline \hline & \multicolumn{3}{c}{ EC electron efficiencies } \\
\hline Method & $\varepsilon_{\mathrm{L} 2}$ & $\varepsilon_{\text {cal }}$ & $\varepsilon_{\text {trk }}$ \\
\hline$A$ & $0.9932 \pm 0.0015$ & $0.8618 \pm 0.0064$ & $0.7320 \pm 0.0089$ \\
$B$ & $0.9980 \pm 0.0008$ & $0.8627 \pm 0.0058$ & $0.7284 \pm 0.0081$ \\
$C$ & $0.9930 \pm 0.0016$ & $0.8645 \pm 0.0064$ & $0.7347 \pm 0.0088$ \\
$D$ & $0.9975 \pm 0.0008$ & $0.8672 \pm 0.0057$ & $0.7328 \pm 0.0080$ \\
\hline \hline
\end{tabular}




\subsubsection{Level-Ø Efficiency}

The Level- $\varnothing$ trigger imposes a universal minimum bias requirement on all events. Its requirements are quite simple: hits must be recorded in both Level- $\varnothing$ counters, and the resultant fast $z$ calculation must be consistent with $\left|z_{v t x}\right|<96.875 \mathrm{~cm}$. In order to calculate the Level- $\varnothing$ efficiency for $W$ and $Z$ events, the Level- $\varnothing$ system logic was modified during Run Ib: events passing the $W \rightarrow e \nu$ trigger (EM1_EISTRKCC_MS) were no longer required to fire the Level- $\varnothing$ trigger, but the latter's decision was saved along with the event. Due to the relatively lower number of $Z$ candidate events, the Level- $\varnothing$ efficiency measured by the $W$ sample is used in both the $W$ and $Z$ cross section calculations. Naturally, this is not a significant shortcoming, since the underlying events in $W$ and $Z$ boson production are essentially identical.

A sample of $W$ events is selected using the cuts outlined in Section 4.2, with the Level- $\varnothing$ requirement removed. Such events are restricted to trigger versions 10.0 and later, where the Level- $\varnothing$ system operated in the mark and pass mode. Many of these events contain more than one interaction. This must be properly taken into account, since any additional interactions will increase the probability that the Level- $\varnothing$ system fires. The overall Level- $\varnothing$ efficiency is then expressed as follows [106, p. 106]:

$$
\varepsilon_{\mathrm{L} \varnothing}(W)=\sum_{n=0}^{\infty} \varepsilon_{\mathrm{L} \emptyset}(W+n) \cdot P(n)
$$

where $\varepsilon_{\mathrm{L} \varnothing}(W)$ is the overall Level- $\varnothing$ efficiency for $W$ events, while $\varepsilon_{\mathrm{L} \varnothing}(W+n)$ and $P(n)$ are the Level- $\varnothing$ efficiency and the probability for a $W$ event accompanied with $n$ minimum bias events, respectively. With each additional minimum bias event, the 
efficiency of the Level- $\varnothing$ system increases, quickly approaching unity. By assuming that $\varepsilon_{\mathrm{L} \varnothing}(W+n)=1$ for $n \geq 2$, Equation 6.7 is re-expressed as:

$$
\varepsilon_{\mathrm{L} \varnothing}(W)=\varepsilon_{\mathrm{L} \emptyset}(W+0) \cdot P(0)+\varepsilon_{\mathrm{L} \emptyset}(W+1) \cdot P(1)+(1 .-P(0)-P(1)) .
$$

A measure of the number of interations in each event is made by using a tool developed specifically for this purpose, MULTIPLE_INTERACTION_TOOL_RUN1 [97]: the probability of multiple interactions is estimated by utilizing information from the Level- $\varnothing$ system, the tracking system, and the calorimeter simultaneously. Based on the $W$ signal sample, $P(0)$ and $P(1)$ are measured to be:

$$
P(0)=0.3717 \pm 0.0019 \quad P(1)=0.6192 \pm 0.0019
$$

while $\varepsilon_{\mathrm{L} \varnothing}(W+0)$ and $\varepsilon_{\mathrm{L} \varnothing}(W+1)$ are:

$$
\varepsilon_{\mathrm{L} \varnothing}(W+0)=\frac{15125}{15701}=0.9633 \pm 0.0015
$$

and

$$
\varepsilon_{\mathrm{L} \varnothing}(W+1)=\frac{31747}{32014}=0.9917 \pm 0.0005
$$

Inserting the above numbers into Equation 6.8, one obtains the overall Level- $\varnothing$ efficiency for $W$ and $Z$ events:

$$
\varepsilon_{\mathrm{L} \varnothing}(W)=\varepsilon_{\mathrm{L} \varnothing}(Z)=0.9860 \pm 0.0050
$$

\subsubsection{Level-2 $\mathbb{E}_{T}$ Trigger Efficiency}

In order to estimate the efficiency of the Level-2 missing transverse energy term, $\not_{T}^{\mathrm{L} 2}$, the $W$ event criteria is applied on events collected with the single electron monitor 
trigger. The latter did not have the $\not_{T}^{\mathrm{L} 2}>15 \mathrm{GeV}$ cut imposed. From a total of 5245 candidate events which were collected with this trigger, 5207 pass the $\not \mathbb{L}_{T}^{\mathrm{L} 2}>15 \mathrm{GeV}$ requirement. Hence the $\mathbb{E}_{T}$ trigger efficiency is:

$$
\varepsilon_{\mathrm{L} 2 m e t}=\frac{5207}{5245}=0.9928 \pm 0.0011
$$

\subsection{Overall $W$ and $Z$ Efficiencies}

As was mentioned in Chapter 4, two different electron selections are used in this analysis: a tight selection is employed in the identification of electrons from $W$ and $Z$ boson decays, while a loose selection is employed in the identification of the second electron in $Z \rightarrow e e$ events. Since the loose cuts have the tracking requirement removed, they form a subset of the tight cuts. The tight electron selection efficiency can then be expressed as:

$$
\varepsilon_{\text {ele }}^{w}=\varepsilon_{\ell} \cdot \varepsilon_{t}
$$

where $\varepsilon_{\text {ele }}^{w}$ is the overall tight electron selection efficiency employed in the selection of $W$ electron events, $\varepsilon_{\ell}$ is the efficiency of the loose cuts employed in the identification of the second electron in $Z$ events, and $\varepsilon_{t}$ is the efficiency of the additional cuts used to make up the tight electron selection. The loose selection include the Level-2 electron trigger term, as well as the calorimeter based requirements. The tight selection further requires a good matching track. Hence, the loose and tight efficiencies can be 
identified as:

$$
\varepsilon_{\text {ele }}^{w}=\underbrace{\varepsilon_{\mathrm{L} 2} \cdot \varepsilon_{c a l}}_{\varepsilon_{\ell}} \cdot \underbrace{\varepsilon_{t r k}}_{\varepsilon_{t}} .
$$

\subsection{1 $W$ Selection Efficiency}

The values for $\varepsilon_{\text {ele }}^{w}$ are computed for each background subtraction method, and are summarized in Table 6.5. Method $D$ is used to quote the central value of the efficiency; the systematic error due to background subtraction is taken to be half the maximum difference between the various methods. Hence, the $W$ selection efficiencies for central (CC) and forward (EC) electrons are:

$$
\varepsilon_{\text {ele }}^{w}(c c)=0.7161 \pm 0.0047 \pm 0.0070 \quad \varepsilon_{\text {ele }}^{w}(e c)=0.6361 \pm 0.0086 \pm 0.0031
$$

where the first error is statistical and the second systematic. To calculate the overall $W$ electron selection efficiency, the $\mathrm{CC}$ and EC results are combined based on the relative acceptance fractions as listed in Table 5.4:

$$
\varepsilon_{\text {ele }}^{w}=0.6921 \pm 0.0100
$$

where the quoted error combines the statistical and systematic errors in quadrature.

The total $W$ selection efficiency is obtained by combining the electron selection efficiency with the Level- $\varnothing$ efficiency and the Level-2 $\not_{T}$ efficiency:

$$
\varepsilon_{\text {tot }}^{w}=\varepsilon_{\mathrm{L} \varnothing} \cdot \varepsilon_{\mathrm{L} 2 m e t} \cdot \varepsilon_{\text {ele }}^{w}=0.6775 \pm 0.0104 .
$$


Table 6.5: Overall $W$ electron selection efficiency (quoted errors are statistical.

\begin{tabular}{ccc}
\hline \hline & \multicolumn{2}{c}{$W$ electron selection efficiency } \\
\hline Method & CC & EC \\
\hline$A$ & $0.7212 \pm 0.0052$ & $0.6322 \pm 0.0086$ \\
$B$ & $0.7104 \pm 0.0051$ & $0.6299 \pm 0.0080$ \\
$C$ & $0.7245 \pm 0.0053$ & $0.6358 \pm 0.0091$ \\
$D$ & $0.7161 \pm 0.0047$ & $0.6361 \pm 0.0086$ \\
\hline \hline
\end{tabular}

\subsubsection{Z Selection Efficiency}

In the $Z$ boson selection, there are two electrons, which have different efficiencies for different cryostats. Hence, three distinct cases of $Z$ electron selection efficiencies are identified:

$$
\begin{aligned}
& \varepsilon_{\text {ele }}^{z}(c c-c c)=\left(\varepsilon_{\ell}^{c}\right)^{2} \cdot\left[2 \varepsilon_{t}^{c}-\left(\varepsilon_{t}^{c}\right)^{2}\right] \\
& \varepsilon_{\text {ele }}^{z}(c c-e c)=\varepsilon_{\ell}^{c} \cdot \varepsilon_{\ell}^{e} \cdot\left(\varepsilon_{t}^{c}+\varepsilon_{t}^{e}-\varepsilon_{t}^{c} \cdot \varepsilon_{t}^{e}\right) \\
& \varepsilon_{\text {ele }}^{z}(e c-e c)=\left(\varepsilon_{\ell}^{e}\right)^{2} \cdot\left[2 \varepsilon_{t}^{e}-\left(\varepsilon_{t}^{e}\right)^{2}\right]
\end{aligned}
$$

where $\varepsilon_{\ell}^{c}$ and $\varepsilon_{t}^{c}$ are the loose and tight selection efficiencies for central electrons, and $\varepsilon_{\ell}^{e}$ and $\varepsilon_{t}^{e}$ are the corresponding efficiencies for forward electrons. The $Z$ electron selection efficiencies are calculated for each background subtraction method, and the results are listed in Table 6.6.

Using method $D$ to quote the central values of the efficiencies, and assigning the systematic error as half the maximum difference between the four methods, the $Z$ electron selection efficiencies are:

$$
\varepsilon_{\text {ele }}^{z}(c c-c c)=0.7943 \pm 0.0054 \pm 0.0094
$$


Table 6.6: Overall $Z$ electron selection efficiency (quoted errors are statistical.

\begin{tabular}{cccc}
\hline \hline & \multicolumn{3}{c}{$Z$ electron selection efficiency } \\
\hline Method & CC-CC & CC-EC & EC-EC \\
\hline$A$ & $0.8034 \pm 0.0056$ & $0.7458 \pm 0.0064$ & $0.6901 \pm 0.0103$ \\
$B$ & $0.7882 \pm 0.0057$ & $0.7383 \pm 0.0057$ & $0.6895 \pm 0.0092$ \\
$C$ & $0.8070 \pm 0.0061$ & $0.7503 \pm 0.0060$ & $0.6958 \pm 0.0105$ \\
$D$ & $0.7943 \pm 0.0054$ & $0.7458 \pm 0.0057$ & $0.6981 \pm 0.0093$ \\
\hline \hline
\end{tabular}

$$
\begin{aligned}
& \varepsilon_{\text {ele }}^{z}(c c-e c)=0.7458 \pm 0.0057 \pm 0.0060 \\
& \varepsilon_{\text {ele }}^{z}(e c-e c)=0.6981 \pm 0.0093 \pm 0.0043 .
\end{aligned}
$$

To calculate the overall $Z$ electron selection efficiency, the CC-CC, CC-EC and ECEC results are combined based on their relative acceptance fractions as listed in Table 5.4, with care taken to properly handle the correlation in the error calculation:

$$
\varepsilon_{\text {ele }}^{z}=0.7650 \pm 0.0086 .
$$

The quoted error combines the statistical and systematic errors in quadrature.

The total $Z$ selection efficiency is obtained by combining the electron selection efficiency with the Level- $\varnothing$ efficiency:

$$
\varepsilon_{\text {tot }}^{z}=\varepsilon_{\mathrm{L} \emptyset} \cdot \varepsilon_{\text {ele }}^{z}=0.7543 \pm 0.0094 .
$$

\subsubsection{Efficiency Ratio}

The primary concern of this analysis is the measurement of the cross section ratio of $W$ and $Z$ electronic decays. Hence, the measurement of the ratio of selection efficiencies is quite important. Fortunately, a large portion of the systematic error in 
the electron selection efficiency ratio cancels since it is correlated ${ }^{2}$. This correlation is taken into account by a simple Monte Carlo program. This program correlates the loose and tight efficiencies by using one single random number in deciding if an electron passes the loose, the tight, or both efficiencies simultaneously. From this the $W$ and $Z$ boson efficiencies, and their ratio, are computed. An ensemble of 10000 such experiments is repeated, and the distribution of all efficiencies are fitted to gaussians. The central value of the efficiencies is obtained from the mean of the gaussian, and the error from its width. The computation of the efficiency ratio is performed for each of the background subtraction methods: the central value is again chosen to be the one from method $D$, while the systematic error is taken to be half the maximum difference between the various methods. The efficiency ratio is measured to be:

$$
\frac{\varepsilon^{z}}{\varepsilon^{w}}=1.113 \pm 0.005 \pm 0.002=1.113 \pm 0.005
$$

The values of this ratio for the four different methods are summarized in Table 6.7.

Table 6.7: Ratio of $W$ and $Z$ selection efficiencies (quoted errors are statistical).

\begin{tabular}{cc}
\hline \hline Method & $\varepsilon^{z} / \varepsilon^{w}$ \\
\hline$A$ & $1.115 \pm 0.005$ \\
$B$ & $1.113 \pm 0.005$ \\
$C$ & $1.116 \pm 0.005$ \\
$D$ & $1.113 \pm 0.005$ \\
\hline \hline
\end{tabular}

\footnotetext{
${ }^{2}$ The efficiency of the Level- $\varnothing$ system cancels completely.
} 


\section{Chapter 7}

\section{Backgrounds}

This chapter discusses the background which contaminates the final $W \rightarrow e \nu$ and $Z \rightarrow e e$ samples. As stated in Chapter 4, electronically decaying vector bosons have very distinct characteristics, so that the application of electron identification cuts provides a clean data sample. However, the choice of the values of the cuts themselves represent a compromise between retaining high efficiency and reducing the background. A small amount of background is still acceptable in the final sample, as long as methods are developed to reliably measure it.

\subsection{Backgrounds in the $W \rightarrow e \nu$ Sample}

The signature of a high- $p_{T}$ electron and large $\mathbb{E}_{T}$ which forms the basis of the $W$ boson selection is quite unique. Nevertheless, there are several sources of instrumental and physics backgrounds which contaminate the final event sample. The largest source arises when a jet in QCD multi-jet events fluctuates electromagnetically and mimics the electron signature, while another is mismeasured giving rise to missing 
transverse energy. Additional backgrounds come from $W \rightarrow \tau \nu$ and $Z \rightarrow \tau \tau$ events with the subsequent decay of a $\tau$ into an electron, $\tau \rightarrow e \nu$. A final source is from $Z \rightarrow e e$ events where one electron is lost in the cracks of the detector, giving rise to $\mathbb{E}_{T}$.

\subsubsection{QCD Multi-jets}

The dominant background source in the $W \rightarrow e \nu$ sample originates from QCD multi-jet events, where one of the jets fluctuates electromagnetically and fakes the electron signature, while another has its energy mismeasured (perhaps most of the energy gets deposited in detector cracks) so that the event has an apparent large amount of $\not_{T}$. The amount of background from this source is measured using a data-based technique, outlined below.

Consider a sample of $M W \rightarrow e \nu$ events which are obtained with a minimal set of cuts (maybe the only requirement is that such a sample passes the $W \rightarrow e \nu$ trigger), labeled the parent sample. If one subsequently imposes a set of tight cuts (maybe the actual $W \rightarrow e \nu$ selection cuts), one obtains two subsamples: a subsample containing $P$ events which passed the tight cuts, and a subsample containing $F$ events which failed. However, the parent sample can be also be divided into two other subsamples: one containing the actual number $R$ of real $W \rightarrow e \nu$ events, and another containing the actual number $B$ of background events. An illustration of the two possible ways of dividing the parent sample is provided in Figure 7.1. 


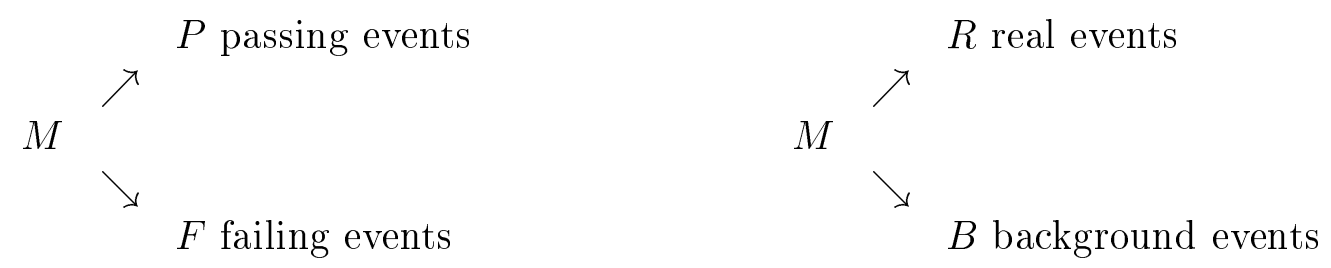

Figure 7.1: Diagram illustrating the two ways to split the parent sample in the determination of QCD multi-jet background to $W \rightarrow e \nu$ events.

With the knowledge of the efficiency and rejection of the tight cuts relative to the loose cuts of the parent sample, one can relate the subsamples $(P, F)$ to the subsamples $(R, B)$ :

$$
\left(\begin{array}{c}
P \\
F
\end{array}\right)=\left(\begin{array}{cc}
\varepsilon_{e} & \varepsilon_{j} \\
1-\varepsilon_{e} & 1-\varepsilon_{j}
\end{array}\right)\left(\begin{array}{c}
R \\
B
\end{array}\right)
$$

where $\varepsilon_{e}$ is the efficiency of the tight cuts (relative to the loose cuts of the parent sample) on electrons, and $\varepsilon_{j}$ is the efficiency of the tight cuts on jets. This later efficiency is nothing more than the inverse of the rejection $r$ of the cuts on jets, $\varepsilon_{j}=r^{-1}$. There are two additional constraints that arise in the construction of the problem, namely the conservation of the total number of events in the parent sample:

$$
M=P+F \quad \text { and } \quad M=R+B .
$$

The QCD multi-jet background in the $W \rightarrow e \nu$ sample is contained in Equation 7.1, as part of the expression for $P$ :

$$
P=\varepsilon_{e} R+\varepsilon_{j} B
$$

The total number of QCD multi-jet background events is

$$
N_{q c d}^{w}=\varepsilon_{j} B
$$


or, alternatively, the background fraction is

$$
f_{q c d}^{w}=\frac{\varepsilon_{j} B}{P}
$$

The actual solution to the background fraction in terms of the known (or measurable) parameters $M, P, \varepsilon_{e}$, and $\varepsilon_{j}$, entails inverting the $2 \times 2$ matrix in Equation 7.1:

$$
\left(\begin{array}{c}
R \\
B
\end{array}\right)=\frac{1}{\varepsilon_{e}-\varepsilon_{j}}\left(\begin{array}{cc}
1-\varepsilon_{j} & -\varepsilon_{j} \\
-\left(1-\varepsilon_{e}\right) & \varepsilon_{e}
\end{array}\right)\left(\begin{array}{c}
P \\
M-P
\end{array}\right) \text {. }
$$

The matrix is invertible as long as its determinant $\varepsilon_{e}-\varepsilon_{j}$ is not identically zero, which is certainly the case since the the electron identification criteria was constructed in such a way as to keep most genuine electrons and to reject most background sources $\left(\varepsilon_{e}>\varepsilon_{j}\right)$. From Equation 7.6, the expression for $B$ is resolved:

$$
B=\frac{\varepsilon_{e} M-P}{\varepsilon_{e}-\varepsilon_{j}}
$$

which, upon substitution into Equation 7.5, yields the expression for the background fraction:

$$
f_{q c d}^{w}=\frac{\varepsilon_{j}}{P} \cdot \frac{\varepsilon_{e} M-P}{\varepsilon_{e}-\varepsilon_{j}} .
$$

In carrying out the method outlined above, the parent sample is chosen to have all the $W \rightarrow e \nu$ selection cuts (see Section 4.2.2), but with the tight electron requirement replaced by a track electron requirement ${ }^{1}$. In this manner, the electron does not have any of the calorimeter based identification criteria, but has a very good track. The tracking requirement is important, since the electron vertex method (Section 3.8.1) is being applied.

\footnotetext{
${ }^{1}$ Please refer to Section 4.1 for the various definitions of electrons (loose,track,tight, etc).
} 
The efficiencies that have to be measured, $\varepsilon_{e}$ and $\varepsilon_{j}$, are the efficiencies of the calorimeter based identification criteria $\left(f_{e m}, \chi_{h m}^{2}\right.$, and $\left.f_{i s o}\right)$ relative to a track electron. The electron efficiency $\varepsilon_{e}$ is easily obtained by using the methods in Chapter 6 . The jet efficiency $\varepsilon_{j}$ is obtained in a similar manner, but from a sample of "electrons" that are dominated by jets. Such a sample can be obtained from a single electron trigger $^{2}$. Figure $7.2 \mathrm{a}$ shows the $\mathbb{E}_{T}$ spectrum of a combined signal and background sample, obtained by requiring one tight electron in the single electron trigger data. In contrast, Figure $7.2 \mathrm{~b}$ shows the $\mathbb{E}_{T}$ spectrum of the background alone, obtained by requiring calorimeter anti-quality cuts $^{3}$ on EM objects in the same data set. The large peak at low misssing $E_{T}$, common in both figures, is clearly due to QCD di-jet events; the broad peak at high missing $E_{T}$ in the signal plot comes from $W \rightarrow e \nu$ signal events. Very few such signal events are expected to populate the $\mathbb{E}_{T}$ region below $15 \mathrm{GeV}$. The electron efficiency for jets, $\varepsilon_{j}$, is measured as the ratio of the number of background events in the signal sample to the number of background events in the parent sample; these background events occupy the $0-15 \mathrm{GeV}$ window of the $\mathbb{E}_{T}$ spectrum.

Table 7.1: Measured parameters used as input to the QCD multi-jet fraction determination in $W \rightarrow e \nu$ events.

\begin{tabular}{ccccc}
\hline \hline & $M$ & $P$ & $\varepsilon_{e}$ & $\varepsilon_{j}$ \\
\hline $\mathrm{CC}$ & 51430 & 47041 & $0.939 \pm 0.003$ & $0.557 \pm 0.027$ \\
$\mathrm{EC}$ & 28281 & 20404 & $0.868 \pm 0.007$ & $0.343 \pm 0.019$ \\
\hline \hline
\end{tabular}

\footnotetext{
${ }^{2} \mathrm{~A}$ single electron trigger without a $\not_{T}$ cut at Level-2 was collected, but heavily prescaled, during the entire run.

${ }^{3}$ The actual requirements were $S_{t r k}<5(10)$ in the $\mathrm{CC}(\mathrm{EC})$ but $f_{\text {iso }}>0.15, \chi_{h m}^{2}>200$.
} 

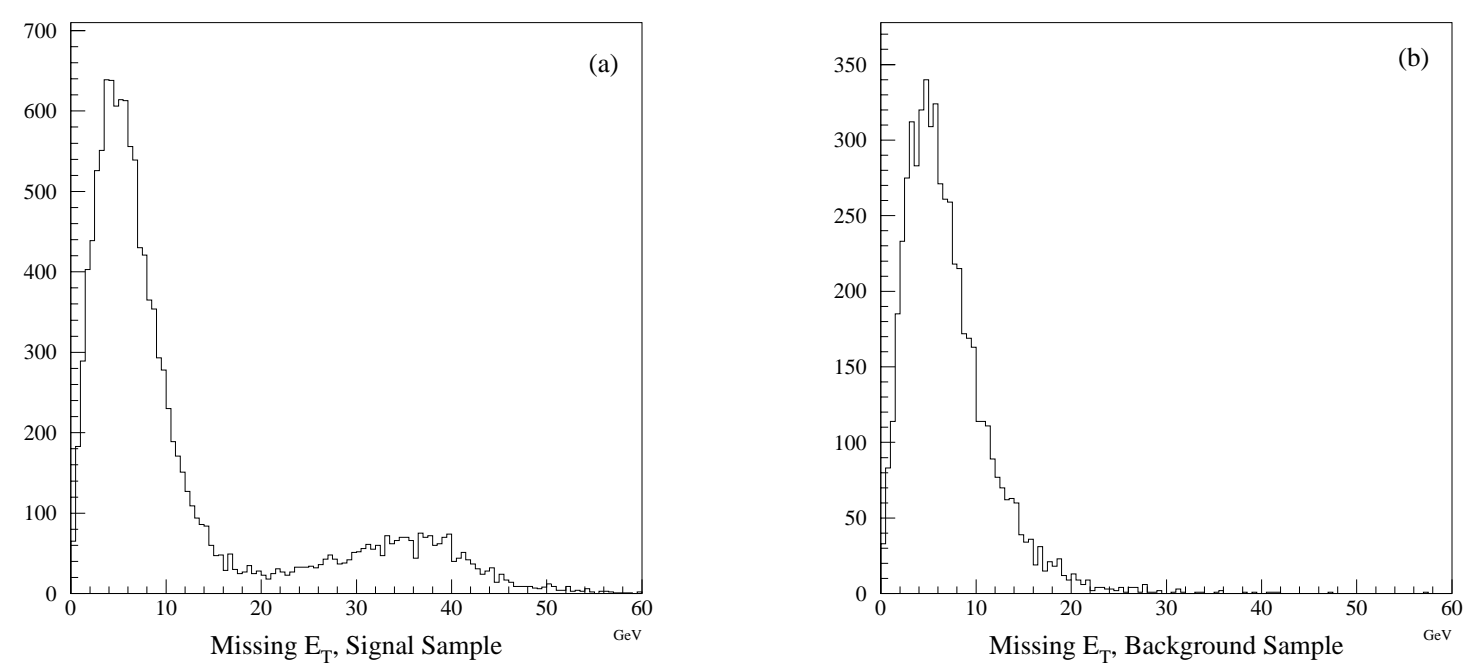

Figure 7.2: $\mathbb{E}_{T}$ spectra for the (a) signal and (b) background event samples in the single electron trigger.

The measured parameters $M, P, \varepsilon_{e}$, and $\varepsilon_{j}$, are summarized in Table 7.1. By using them as input to Equation 7.8, the background fractions for central and forward $W \rightarrow e \nu$ events are measured to be:

$$
f_{q c d}^{w}(c c)=(4.0 \pm 0.7) \% \quad \text { and } \quad f_{q c d}^{w}(e c)=(13.3 \pm 1.1) \%
$$

which amount to an overall QCD multi-jet background fraction of:

$$
f_{q c d}^{w}=(6.8 \pm 0.8) \%
$$

The quoted error contains a statistical part (computed by assuming binomial errors), and a systematic part which comes from the error on the measured efficiencies $\varepsilon_{e}$ and $\varepsilon_{j}$. It should be noted that this background fraction is a fraction of the $W \rightarrow e \nu$ final candidate sample, and not a fraction of the real $W$ boson events. 


\subsection{2 $W \rightarrow \tau \nu$}

Another source of background comes from $W \rightarrow \tau \nu$ events, where the $\tau$ decays electronically, $\tau \rightarrow e \nu$. These events constitute a physics background to $W \rightarrow e \nu$ events, since they contain the same final state leptons. However, the final state electrons are not the decay product of the vector boson, but rather of the $\tau$. The resultant electron $E_{T}$ spectrum tends to be much softer: it does not exhibit the characteristic Jacobian peak at a value of $M_{W} / 2$, but rather peaks at low energies and falls off rapidly (see Figure 7.3). With the $25 \mathrm{GeV} E_{T}$ cut that is imposed on the electron, the kinematic acceptance of $W \rightarrow \tau \nu$ events is greatly restricted. In addition, the amount which contaminates $W \rightarrow e \nu$ events is further reduced by the $\tau \rightarrow e \nu$ branching ratio of $17.8 \%$ [18].

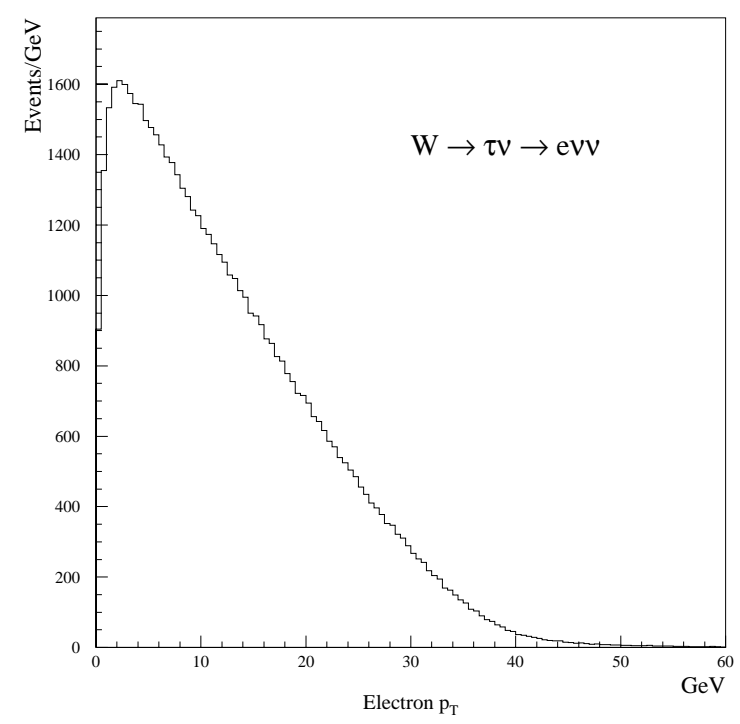

Figure 7.3: Electron $E_{T}$ distribution from $W \rightarrow \tau \nu \rightarrow e \nu \nu$ events. 
The amount of background is determined by using the parametric Monte Carlo used in Chapter 5 (see Section 5.1.3.2). The geometric and kinematic acceptance, $\mathcal{A}_{w}^{\tau}$, of the $W \rightarrow \tau \nu \rightarrow e \nu \nu$ process, is calculated in the same way as the $W \rightarrow e \nu$ acceptance, $\mathcal{A}_{w}^{e}$. The background fraction is then given by the ratio of the two acceptances, modified by the $\tau \rightarrow e \nu$ branching fraction:

$$
f_{\tau}^{w}=\operatorname{Br}(\tau \rightarrow e \nu) \cdot \frac{\mathcal{A}_{w}^{\tau}}{\mathcal{A}_{w}^{e}}
$$

if $W$ bosons decay equally into electrons and taus (lepton universality). Using $\operatorname{Br}(\tau \rightarrow e \nu)=(17.8 \pm 0.1) \%$, the background fraction is measured to be:

$$
f_{\tau}^{w}=(2.1 \pm 0.2) \%
$$

where the error is dominated by Monte Carlo statistics since all other systematic errors cancel in the ratio, and the error on the branching fraction is negligible. It is important to note that this fraction is a fraction of the true $W \rightarrow e \nu$ events, and not a fraction of the final candidate sample.

\subsection{3 $Z \rightarrow e e$}

In $Z \rightarrow e e$ decays, it is possible for one of the electrons to enter an uninstrumented region of the detector. Not only will this electron have a small probability of being reconstructed, but the lost energy will show up as sizable missing transverse energy in the event. Under these conditions, the $Z \rightarrow e \notin$ events will have an identical signature to $W \rightarrow e \nu$ events, and will end up contaminating it. Such a background is difficult to measure using data-based techniques. Instead, it is calculated using a Monte 
Carlo. The parametric detector Monte Carlo of Chapter 5 is not adequate, since a detailed simulation of the cracks is needed. Hence, this study is performed using DØGEANT [98], a detailed simulation of the DØ detector based on the GEANT [77] Monte Carlo.

A sample of $9,970 Z \rightarrow e e$ events is prepared using HERWIG, then processed through DØGEANT and DØRECO. These events are subsequently overlayed with zero-bias data taken at a luminosity $\mathcal{L}=3 \cdot 10^{30} \mathrm{~cm}^{-2} \mathrm{~s}^{-1}$. The $W \rightarrow e \nu$ selection criteria are applied to this sample, and 295 events survive. The background fraction is then the product of the ratio of this DØGEANT based acceptance $\left(\mathcal{A}_{z \rightarrow w}\right)$ to the CMS acceptance $\left(\mathcal{A}_{w}\right.$, and the ratio of $Z$ to $W$ inclusive cross sections times branching fractions:

$$
f_{z}^{w}(e)=\frac{\sigma(p \bar{p} \rightarrow Z \rightarrow e e)}{\sigma(p \bar{p} \rightarrow W \rightarrow e \nu)} \cdot \frac{\mathcal{A}_{z \rightarrow w}}{\mathcal{A}_{w}}=\frac{\sigma(p \bar{p} \rightarrow Z \rightarrow e e)}{\sigma(p \bar{p} \rightarrow W \rightarrow e \nu)} \cdot \frac{\frac{3792}{50668}}{\mathcal{A}_{w}}
$$

The ratio of production cross sections $\sigma(p \bar{p} \rightarrow Z \rightarrow e e) / \sigma(p \bar{p} \rightarrow W \rightarrow e \nu)$ is taken from theoretical calculations. The assumed value of 0.1 is very close to previous experimental observations. The value of $\mathcal{A}_{w}$ is taken from Chapter 5: $47.1 \%$. Finally, $\mathcal{A}_{z \rightarrow w}$ is assigned a conservative relative error of $10 \%$, which covers the Monte Carlo statistics, and any difference between the CMS and HERWIG generators. The background fraction is measured to be:

$$
f_{z}^{w}(e)=(0.59 \pm 0.06) \%
$$

The quoted error is dominated by the error on the HERWIG acceptance. It is important to note once again that this background fraction is a fraction of the true $W \rightarrow e \nu$ events, and not the final candidate sample. 


\subsection{4 $Z \rightarrow \tau \tau$}

The process $Z \rightarrow \tau \tau$ with one $\tau$ decaying electronically, $\tau \rightarrow e \nu$, is also a physics background to $W \rightarrow e \nu$ events. This background is also estimated from $Z \rightarrow \tau \tau$ events prepared with HERWIG, then processed though DØGEANT and DØRECO. From a sample of $31,485 Z \rightarrow \tau \tau$ events, where one of the $\tau$ leptons is forced to decay to an electron, 750 events survive the $W$ selection criteria.

The amount of background is extracted in exactly the same fashion as the $Z \rightarrow e e$ background. However, one must include the $\tau \rightarrow e \nu$ branching fraction of $17.8 \%$ :

$$
f_{z}^{w}(\tau)=\frac{\sigma(p \bar{p} \rightarrow Z \rightarrow e e)}{\sigma(p \bar{p} \rightarrow W \rightarrow e \nu)} \cdot \frac{\mathcal{A}_{z \rightarrow w}}{\mathcal{A}_{w}} \cdot\left(2 \operatorname{Br}(\tau \rightarrow e \nu)-\operatorname{Br}(\tau \rightarrow e \nu)^{2}\right)
$$

By assigning a conservative $10 \%$ error to $\mathcal{A}_{z \rightarrow w}$, one obtains a background fraction:

$$
f_{z}^{w}(\tau)=(0.16 \pm 0.02) \%
$$

which is a fraction of the true number of $W \rightarrow e \nu$ events, and not the final candiate sample.

\subsection{Backgrounds in the $Z \rightarrow e e$ Sample}

The signature of two isolated high- $p_{T}$ electrons which forms the basis of the $Z$ boson selection is quite unique. Nevertheless, there exist instrumental and physics backgrounds which contaminate the final event sample. The largest source stems from jets which fluctuate electromagnetically, forming a QCD multi-jet background. 
In addition, the Drell-Yan mechanism, and the $Z \rightarrow \tau \tau \rightarrow e e \nu \nu$ process, can contribute to the production of two isolated high- $p_{T}$ electrons.

\subsubsection{QCD Backgrounds}

The dominant background source in the $Z \rightarrow e e$ event sample consists of QCD processes: di-jet production, where both jets fluctuate electromagnetically, and direct "photon + jet" production, where the photon satisfies the loose electron criteria, and the jet masquerades as the second electron. Since the invariant mass distribution of the electrons from $Z$ boson decays exhibits a well defined resonance peak, and the spectrum from QCD sources is exponentially falling, a data-based technique is utilized to estimate the amount of QCD background. The technique, outline below, exploits the difference in shape from each sample by performing a maximum likelihood fit of all samples to the actual data, and extracts the relative contribution of each.

The invariant mass shape of signal events is obtained from the PYTHIA physics generator [99]. The process $p \bar{p} \rightarrow Z / \gamma^{\star} \rightarrow e e$ is generated, which contains the pure $Z$, Drell-Yan, and interference contributions to the production cross section. The generated electron 4-vectors are smeared with the detector resolution and QED radiation effects using the parametric detector Monte Carlo from Chapter 5. After fiducial and geometric cuts are applied, the resultant mass distribution, $N_{Z / \gamma}(m)$, is shown in Figure 7.4. 


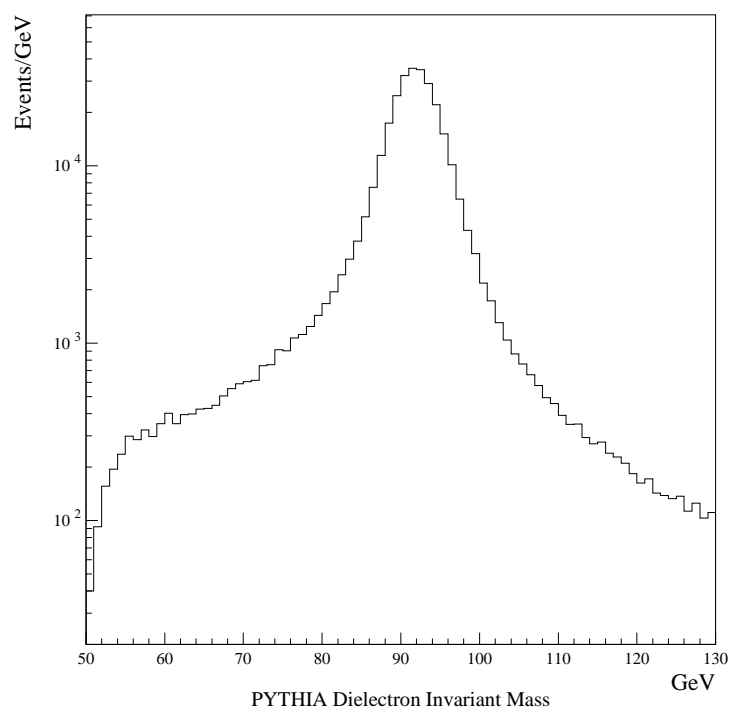

Figure 7.4: The $Z / \gamma$ mass distribution used in fitting the QCD background to $Z \rightarrow e e$ events.

The invariant mass shape of background events is obtained from the DØ direct photon group [100]. The "photon + jet" selection is highly contaminated with dijet events ${ }^{4}$ making it ideal as a QCD background model. The spectrum, labeled $N_{\gamma-j e t}(m)$, is shown in Figure 7.5.

To figure out the amount of QCD background contaminating the $Z \rightarrow e e$ sample, the invariant mass distribution of the data, $N_{\text {data }}(m)$, is fit to the sum of the signal and background distributions:

$$
N_{\text {data }}(m)=c_{1} \cdot N_{Z / \gamma}+c_{2} \cdot N_{\gamma-j e t}
$$

where $c_{1}$ and $c_{2}$ are the normalization factors of the signal and background respectively. These two factors are not independent since their sum must add up to unity. A binned maximum likelihood fit [101, chap. 9] is performed using the function HMCMLL

\footnotetext{
${ }^{4}$ The sample is composed of roughly $50 \%$ photon-jet events, and $50 \%$ di-jet events.
} 


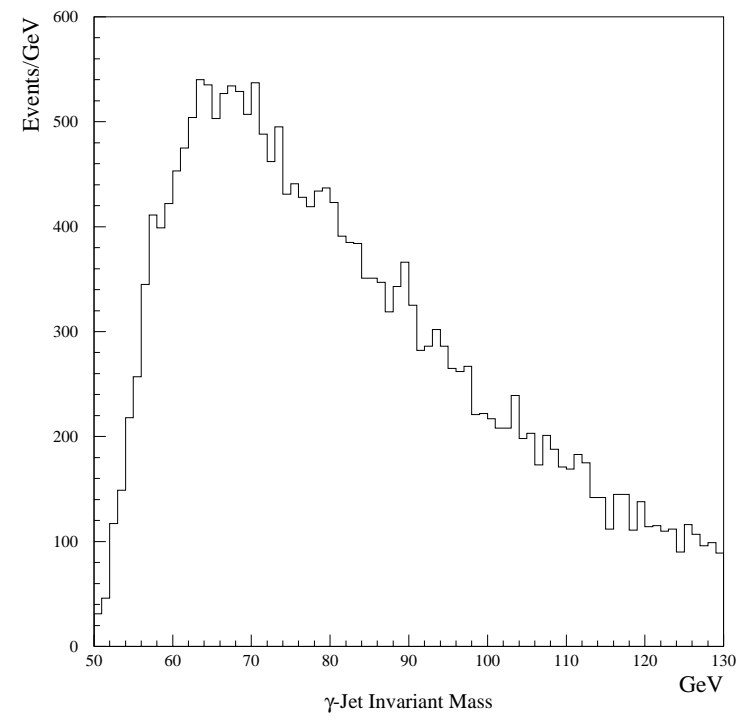

Figure 7.5: The QCD background distribution from "photon + jet" events, used in fitting the QCD background to $Z \rightarrow e e$ events.

provided in the HBOOK statistical and histogramming package [102]. This function takes the three distributions for the data, the theoretical prediction, and the background as input, and returns the values of $c_{1}$ and $c_{2}$. The QCD background fraction is then expressed as:

$$
f_{q c d}^{z}=c_{2} \cdot \frac{N_{\text {data }}^{t o t}}{N_{\gamma-j e t}^{t o t}} \cdot \frac{N_{\gamma-j e t}^{75-105}}{N_{\text {data }}^{75-105}}
$$

The fit, performed in the invariant mass window $60-120 \mathrm{GeV} / \mathrm{c}^{2}$, yields:

$$
f_{q c d}^{z}=(4.1 \pm 0.4) \%
$$

For this value, the $Z \rightarrow e e$ data is compared to the sum of the signal and predicted background in Figure 7.6. The agreement is quite good, with a $\chi^{2}$ per degree of freedom of 1.15. This background fraction is a fraction of the $Z \rightarrow e e$ candidate sample, and not of the real $Z$ boson events. 


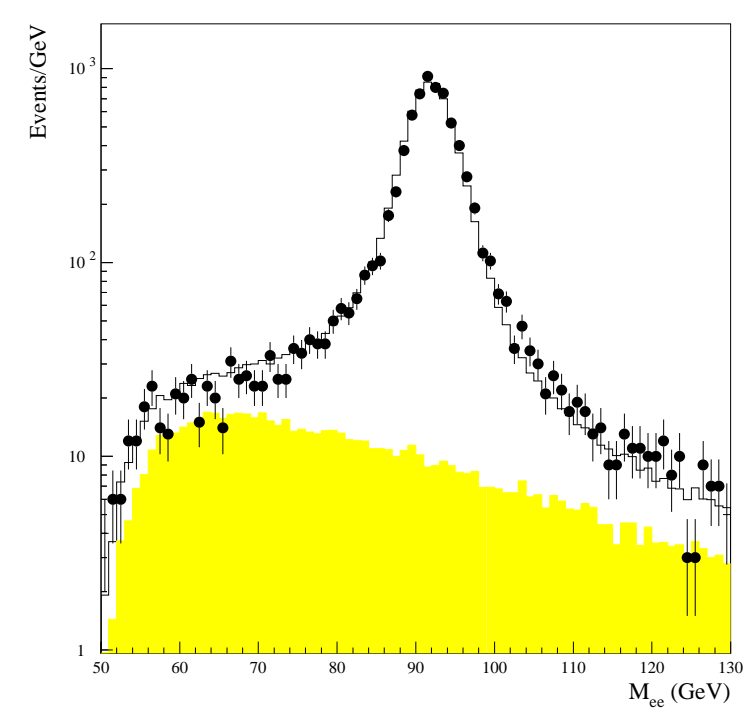

Figure 7.6: Comparison of the $Z \rightarrow e e$ invariant mass distribution for the data (dots), the predicted background (shaded), and the sum of Monte Carlo signal and predicted background (histogram).

\subsubsection{Drell-Yan and $Z / \gamma^{\star}$ Interference}

The physics underlying $p \bar{p} \rightarrow Z \rightarrow e e$ production is exactly the same if the $Z$ is replaced by an off-shelf photon, $\gamma^{\star}$. The actual mechanism is $p \bar{p} \rightarrow Z / \gamma^{\star} \rightarrow e e$ with an amplitude $M_{Z}$, an amplitude $M_{\gamma^{\star}}$, and an interference term $M_{Z-\gamma^{\star}}$ contributing to the cross section. However, in order to measure a pure $Z \rightarrow e e$ cross section, the data sample must be corrected for the contribution of events from the Drell-Yan $\left(\left|M_{\gamma^{\star}}\right|^{2}\right)$ and interference $\left(\left|M_{Z-\gamma^{\star}}\right|^{2}\right)$ terms.

Two 1,000,000 event samples are generated using PYTHIA: one using the full $Z-\gamma^{\star}$ matrix elements (this includes the interference term), and another using the pure $Z$ matrix element alone. The samples, generated with a lower kinematic limit $q^{2}>30 \mathrm{GeV} / \mathrm{c}^{2}$, correspond to cross sections of $234.1 \mathrm{nb}$ for the full $Z-\gamma^{\star}$ matrix, 
and to $179.3 \mathrm{nb}$ for the pure $Z$ matrix. The electron 4 -vectors are smeared using the parametric detector Monte Carlo from Chapter 5, then all kinematic and fiducial cuts are applied: the number of surviving events are 287,678 and 371,330 for $Z / \gamma^{\star} \rightarrow e e$ and $Z \rightarrow e e$ production, respectively.

To determine the fraction of events, $f_{d-y}^{z}$, originating from the Drell-Yan and interference terms, the ratio of the cross sections of the two samples inside the $75-105$ $\mathrm{GeV} / \mathrm{c}^{2}$ invariant mass window is calculated:

$$
1+f_{d-y}^{z}=\frac{\left[\sigma\left(p \bar{p} \rightarrow Z / \gamma^{\star} \rightarrow e e\right)\right]_{75-105}}{[\sigma(p \bar{p} \rightarrow Z \rightarrow e e)]_{75-105}}=\frac{\frac{287678}{100000}}{\frac{371330}{100000}} \cdot \frac{234.1 \mathrm{nb}}{179.3 \mathrm{nb}}=1.012
$$

The statistical error associated with this result is negligible. However, the cross sections could be generator dependent. Hence, this procedure is repeated with events generated using ISAJET, and the results are found to be consistent. In addition, variations of $f_{d-y}^{z}$ due to a variation in the lower limit of $q^{2}$ were found to be small. Therefore, the fraction of background due to Drell-Yan and the interference term is:

$$
f_{d-y}^{z}=(1.2 \pm 0.1) \%
$$

where the quoted error is dominated by the systematic error due to the generator and $q^{2}$ variations. This fraction is a fraction of the real $Z \rightarrow e e$ events, and not a fraction of the signal sample.

Another potential source of background is the Drell-Yan production of taus, $p \bar{p} \rightarrow \gamma^{\star} \rightarrow \tau \tau$, with the taus subsequently decaying to electrons, $\tau \rightarrow e \nu$. Considering the soft $E_{T}$ spectrum of the electrons, as well as the two electronic branching ratios of the $\tau$, this background is negligible and is safely ignored. 


\subsection{3 $Z \rightarrow \tau \tau$}

The final source of background comes from the $Z \rightarrow \tau \tau$ process, where both taus decay electronically. The decay rate of $Z$ bosons into taus is identical to its decay rate to electrons ${ }^{5}$. However the combination of a soft electron $E_{T}$ spectrum and the additional $(17 \%)^{2}$ factor from the $\tau \rightarrow e \nu$ branching fraction make this background negligible. This is verified by generating 900 ISAJET $Z \rightarrow \tau \tau \rightarrow e e \nu \nu$ events, and passing them through DØGEANT and DØRECO. After the geometric and kinematic cuts are imposed, 17 events survive. The invariant mass window cut $\left(75<M_{e e}<\right.$ $105 \mathrm{GeV} / \mathrm{c}^{2}$ ) reduces the sample to one single event. Taking into consideration the $(17 \%)^{2}$ branching ratio factors, the acceptance of $Z \rightarrow \tau \tau \rightarrow e e \nu \nu$ is indeed found to be negligible; thus this source of background can be safely ignored.

\footnotetext{
${ }^{5}$ Again, using lepton universality.
} 


\section{Chapter 8}

\section{Luminosity}

The final ingredient required for the measurement of any cross section is the determination of the integrated luminosity, a measure of the exposure of the detector to $p \bar{p}$ interactions. It is essential to measure this quantity as accurately as possible, since any error on the luminosity translates directly into an error on the cross section. However, it is helpful to note that the error due to the luminosity cancels out in the measurement of the ratio of two cross sections. In calculating the luminosity, a determination of the total number of $p \bar{p}$ interactions used in the analysis, as well as the total $p \bar{p}$ cross section visible to the triggering system, are needed. In addition, knowledge of all sources of dead-time, as well as a careful handling and bookkeeping of the data, are required.

\subsection{Determination of Instantaneous Luminosity}

The instantaneous luminosity $\mathcal{L}$ at $\mathrm{D} \varnothing$ is measured using the Level- $\varnothing$ trigger, where hard-core, inelastic $p \bar{p}$ collisions are detected with high efficiency. The measured 
instantaneous luminosity $\mathcal{L}$ is proportional to the counting rate $R_{\mathrm{L} \varnothing}$ of the Level- $\varnothing$ system:

$$
\mathcal{L}=\frac{R_{\mathrm{L} \varnothing}}{\sigma_{\mathrm{L} \emptyset}}
$$

where $\sigma_{\mathrm{L} \emptyset}$ is the $p \bar{p}$ cross section subtended by the counters of the Level- $\varnothing$ system. This cross section is known as the Level- $\varnothing$ monitor constant, and its calculation will be described in Section 8.2.

For any given crossing, this equation is strictly correct if the counting rate corresponds to the interaction rate. This is the case at low enough luminosities, where single interaction events are dominant. However, as the luminosity increases, so does the proportion of multiple interactions per crossing. The counting rate $R_{\mathrm{L} \varnothing}$ does not distinguish between crossings with a single or multiple interactions, counting only once for each crossing with one or more interactions. Therefore, the direct proportionality in Equation 8.1 breaks down and needs to get corrected.

\subsubsection{Multiple Interaction Correction}

The multiple interaction correction can be calculated using Poisson statistics. At a given instantaneous luminosity $\mathcal{L}$, the average number of interactions per beam crossing, $\bar{n}$, is given by [103]:

$$
\bar{n}=\mathcal{L} \tau \sigma_{\mathrm{L} \varnothing}
$$

where $\tau=3.5 \mu \mathrm{s}$ is the time interval beam crossings. This can be rewritten as

$$
\mathcal{L}=\frac{\bar{n}}{\tau \sigma_{\mathrm{L} \varnothing}}
$$


The probability of having $n$ interactions in one crossing, $\mathcal{P}_{n}$, follows from Poisson statistics, and is given by:

$$
\mathcal{P}_{n}=\frac{\mu}{n !} e^{-\mu}
$$

where $\mu$ is just $\bar{n}$, the average number of interactions per crossing. Since the counting rate does not distinguish between single and multiple interactions, it becomes proportional to the probability of having one or more interactions $\left(\mathcal{P}_{n \geq 1}\right)$ :

$$
\mathcal{P}_{n \geq 1}=1-\mathcal{P}_{0}=1-e^{-\bar{n}}=\mathcal{L} \tau \sigma_{\mathrm{L} \varnothing}=\tau R_{\mathrm{L} \varnothing}
$$

where $P_{0}=e^{-\bar{n}}$ is the Poisson probability of having a crossing with no interactions. This can be used to write $\bar{n}$ as:

$$
\bar{n}=-\ln \left(1-\tau R_{\mathrm{L} \varnothing}\right)
$$

which gets substituted into Equation 8.3 to yield:

$$
\mathcal{L}=\frac{-\ln \left(1-\tau R_{\mathrm{L} \varnothing}\right)}{\tau \sigma_{\mathrm{L} \varnothing}}
$$

This calculation now takes into account the multiple interactions within each beam crossing. However, during normal Tevatron operation, there are six bunches of protons and six bunches of antiprotons giving rise to a total of six distinct $p \bar{p}$ bunchpairs for any given interaction point. Since the bunches tend to have an unequal population of protons (or antiprotons), the multiple interaction probability becomes bunch-pair dependent. To deal with this problem, the luminosity is then measured independently for each bunch-pair and the result is summed. The calculation for each bunch-pair is identical to Equation 8.7 except for a crossing time $\tau$ value which is six times greater. 


\subsection{The Level- $\varnothing$ Monitor Constant $\sigma_{\mathrm{L} \varnothing}$}

In order to calculate the luminosity, it is necessary to know the total $p \bar{p}$ cross section that is visible to the Level- $\varnothing$ counters. This total cross section can be separated into two pieces:

$$
\sigma_{t o t}=\sigma_{e l}+\sigma_{\text {inel }}
$$

where $\sigma_{e l}$ and $\sigma_{\text {inel }}$ are the contribution of elastic and inelastic processes respectively. Furthermore, the inelastic part can be separated into a hard-core, a single-diffractive, and a double-diffractive piece:

$$
\sigma_{i n e l}=\sigma_{H C}+\sigma_{S D}+\sigma_{D D}
$$

A diffractive process is one where the incident $p$ (or $\bar{p}$ ) is excited by vacuum-exchange

to a high mass nucleon state with essentially unchanged energy [5, p. 381], which then decays into a shower of hadrons. Diffraction is characterized by low- $p_{T}$ hadrons, which are emitted in the very forward direction. Single diffraction processes are ones which involve either the incident $p$ or the incident $\bar{p}$, while double diffraction involves both. In contrast, the hard core scattering processes are characterized by a very inelastic high-momentum transfer, and are the processes of interest in this analysis. The Level- $\varnothing$ system is designed to be very efficient at detecting hard core inelastic collisions. The requirement of coincidental hits in the Level- $\varnothing$ counters results in very few elastic or diffractive events. The Level- $\varnothing$ monitor constant $\sigma_{\mathrm{L} \varnothing}$ is obtained by applying the measured efficiencies and acceptances of the Level- $\varnothing$ system to the various components of the total $p \bar{p}$ cross section at $\sqrt{s}=1.8 \mathrm{TeV}$. The calculation is 
given by:

$$
\sigma_{\mathrm{L} \varnothing}=\epsilon_{\mathrm{L} \varnothing} f_{\text {halo }} f_{M S D} \sum_{i} \mathcal{A}_{i} \sigma_{i} \quad(i=S D, D D, H C)
$$

where $\epsilon_{\mathrm{L} \varnothing}$ is the efficiency of the Level- $\varnothing$ system, $\mathcal{A}_{i}$ is the geometric acceptance in the Level- $\varnothing$ array of counters for each process (single-diffractive, double-diffractive and hard-core), each having a cross section $\sigma_{i}$, and $f_{\text {halo }}$ and $f_{M S D}$ are luminosity dependent corrections that deal with beam halo and multiple single-diffractive interactions, respectively.

\subsubsection{Inelastic Scattering Cross Sections}

The total $p \bar{p}$ cross section $\sigma_{p \bar{p}}$, as well as its elastic, inelastic, and single diffractive components, have been measured independently by Fermilab experiments E710 [104] and CDF [105], and are listed in Table 8.1. DØ forms a 'world average' weighted mean of the two measurements. Because of the large difference between the two experiments, the error has been scaled by $\chi$ taken from the $\chi^{2}$ probability that the two values are the same within their quoted errors.

Table 8.1: CDF and E710 $p \bar{p}$ total cross section components and their 'World Average'.

\begin{tabular}{clccccc}
\hline \hline & & $\begin{array}{c}\text { CDF } \\
{[\mathrm{mb}]}\end{array}$ & $\begin{array}{c}\text { E710 } \\
{[\mathrm{mb}]}\end{array}$ & $\begin{array}{c}\text { Combined } \\
{[\mathrm{mb}]}\end{array}$ & $\begin{array}{c}\text { Error } \\
\text { scale }\end{array}$ & $\begin{array}{c}\text { Average } \\
{[\mathrm{mb}]}\end{array}$ \\
\hline \multirow{2}{*}{$\sigma_{e l}$} & Mean & 19.70 & 16.6 & 19.0 & & 19.02 \\
& Error & 0.85 & 1.6 & 0.8 & 1.7 & 1.28 \\
\hline \multirow{2}{*}{$\sigma_{\text {inel }}$} & Mean & 60.3 & 55.5 & 58.9 & & 58.94 \\
& Error & 1.40 & 2.2 & 1.2 & 1.85 & 2.19 \\
\hline \multirow{2}{*}{$\sigma_{S D}$} & Mean & 9.46 & 11.7 & 9.5 & & 9.54 \\
& Error & 0.44 & 2.3 & 0.4 & 1.0 & 0.43 \\
\hline \hline
\end{tabular}


The double-diffractive part of the cross section, $\sigma_{D D}$, is not directly measured, but rather derived from the elastic, $\sigma_{e l}$, and single-diffractive, $\sigma_{S D}$, pieces. The calculation uses factorization (see Figure 8.1) to approximate the equivalence of the ratio:

$$
\frac{\sigma_{D D}}{\sigma_{S D}} \approx \frac{\sigma_{S D}}{\sigma_{e l}}
$$
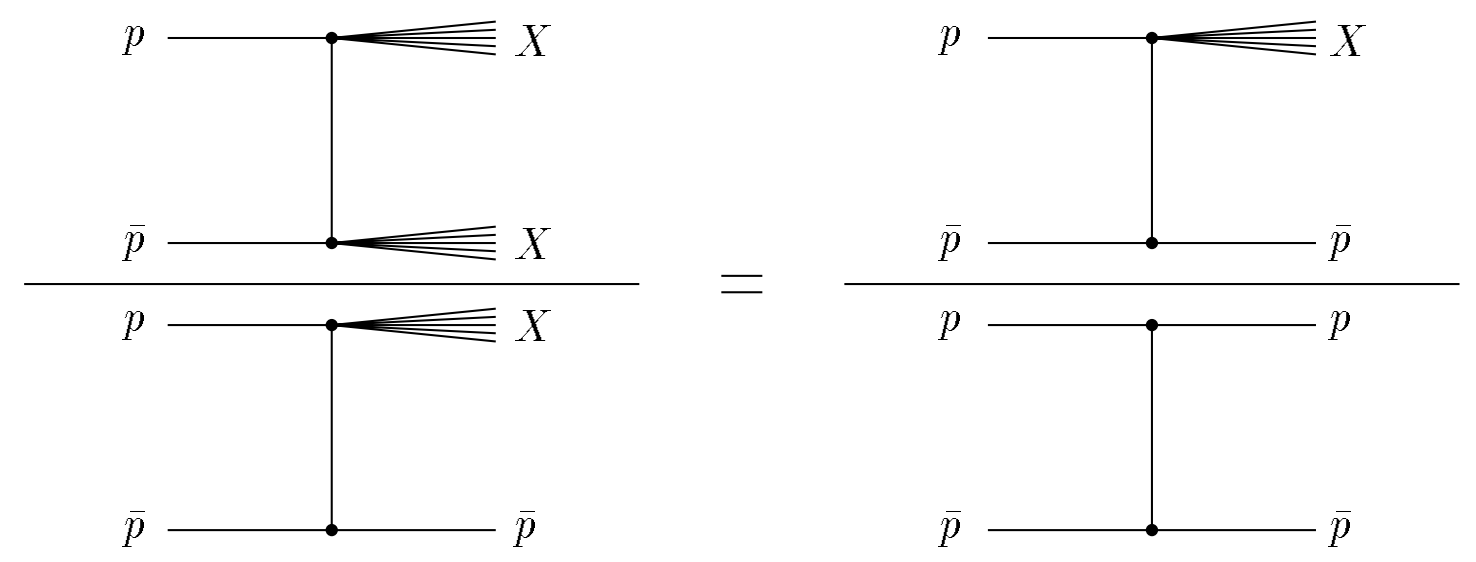

Figure 8.1: Schematic diagram illustrating the factorization principle used in calculating the double-diffractive cross section $\sigma_{D D}[106$, p. 138].

Since the single-diffractive cross section from Table 8.1 contains equal contributions from either the $p$ or $\bar{p}$ diffracting, only half of it should be used in Equation 8.11. The expression for the double-diffractive cross section then becomes:

$$
\sigma_{D D}=\frac{\sigma_{S D}^{2}}{4 \sigma_{e l}}
$$

The calculation of the double-diffractive cross section is done for both the E710 and CDF measurements of the single diffractive and elastic cross sections. A 'world average' weighted mean and error are calculated using the prescription outlined above, 
and the results are listed in Table 8.2. Despite the good agreement with the theoretical calculations of Goulianos [107], a 10\% systematic error is added to the final average to account for the factorization assumption.

Table 8.2: Double-diffractive cross section calculation.

\begin{tabular}{ccccccc}
\hline \hline & & $\begin{array}{c}\text { CDF } \\
{[\mathrm{mb}]}\end{array}$ & $\begin{array}{c}\text { E710 } \\
{[\mathrm{mb}]}\end{array}$ & $\begin{array}{c}\text { Combined } \\
{[\mathrm{mb}]}\end{array}$ & $\begin{array}{c}\text { Fact. Error } \\
{[\mathrm{mb}]}\end{array}$ & $\begin{array}{c}\text { Average } \\
{[\mathrm{mb}]}\end{array}$ \\
\hline \multirow{2}{*}{$\sigma_{D D}$} & Mean & 1.14 & 2.1 & 1.15 & & 1.15 \\
& Error & 0.12 & 0.8 & 0.12 & 0.115 & 0.17 \\
\hline \hline
\end{tabular}

The hard-core component of the inelastic cross section, which contains all the relevant physics processes, can now be deduced by subtracting the diffractive pieces from the total inelastic cross section:

$$
\sigma_{H C}=\sigma_{i n e l}-\sigma_{S D}-\sigma_{D D}
$$

The result is $\sigma_{H C}=48.25 \pm 2.23 \mathrm{mb}$.

\subsubsection{Level- $\varnothing$ Acceptances for Inelastic Scattering Processes}

The acceptances for the inelastic components of the $p \bar{p}$ cross section are calculated using two independent Monte Carlo generators. The MBR [108] Monte Carlo is based on CDF multiplicity studies and the DTUJET [109][110] is based on dual parton model calculations. Each Monte Carlo can generate single-diffractive, doublediffractive, and hard-core events separately. A 1000 event data sample was generated for each process using both generators (for a total of six samples). Each sample was 
subjected to a full detector simulation (DØGEANT) followed by the reconstruction program (DØRECO). For each sample, the measured acceptance was the fraction of events with at least one charged particle passing through each of the north and south Level- $\varnothing$ counter arrays.

The two generators were in fairly good agreement. In addition, cross-checks were performed against zero-bias data ${ }^{1}$, and the agreement was fairly good. The final acceptance is calculated by averaging the results of the two generators, and a systematic error of half the difference is assigned. The acceptance results are summarized in Table 8.3.

Table 8.3: Results of the Monte Carlo Level- $\varnothing$ acceptances.

\begin{tabular}{cccc}
\hline \hline & MBR & DTUJET & Combined \\
\hline $\mathcal{A}_{S D}[\%]$ & $20.5 \pm 1.3$ & $9.7 \pm 0.9$ & $15.1 \pm 0.8 \pm 5.4$ \\
\hline $\mathcal{A}_{D D}[\%]$ & $68.5 \pm 1.5$ & $74.7 \pm 1.6$ & $71.6 \pm 1.1 \pm 3.1$ \\
\hline $\mathcal{A}_{H C}[\%]$ & $95.2 \pm 0.7$ & $99.0 \pm 0.7$ & $97.1 \pm 0.5 \pm 1.9$ \\
\hline \hline
\end{tabular}

\subsubsection{The Level- $\varnothing$ Trigger Efficiency}

The Level- $\varnothing$ efficiency, $\epsilon_{\mathrm{L} \varnothing}$, is defined as the efficiency with which the Level- $\varnothing$ trigger fires on a single, inelastic interaction. It is determined using zero-bias data samples. Events with no in-time hits on either array of the Level- $\varnothing$ system are used to measure the pedestal of the Level- $\varnothing$ counters. To study the efficiency for one array, events are selected such that in-time hits are present on the opposite array:

\footnotetext{
${ }^{1}$ Zero-bias data are collected on random beam crossings: the trigger relies solely on the crossing time of the beam rather than on the detected presence of an interaction.
} 
the pedestal is subtracted, and the remaining fraction of events that have a good fast $z$ signal is taken to be the efficiency for that array. The final Level- $\varnothing$ efficiency is obtained by taking the product of the efficiencies of the north and south arrays, The effects of multiple interactions are accounted for using Poisson statistics as discussed in Section 8.1.1. The results are shown in Figure 8.2. Despite the precautions taken to account for any instantaneous luminosity dependence, the results still exhibit a residual effect. The data are fitted to a line and the result is $\epsilon_{\mathrm{L} \varnothing}=0.897+0.00124 \mathcal{L}$. This fitted slope is used in the determination of the instantaneous luminosity. By using the instantaneous luminosity profile of the complete data sample (Figure 8.3), the luminosity weighted average of the Level- $\varnothing$ trigger efficiency is $\epsilon_{\mathrm{L} \varnothing}=0.907 \pm 0.017$.

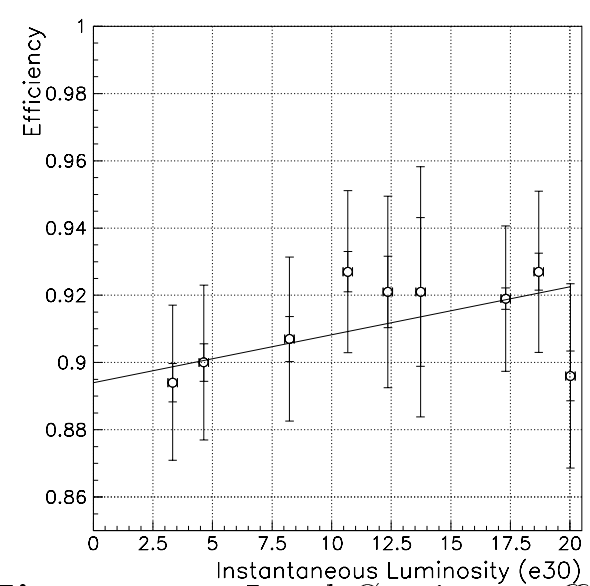

Figure 8.2: Level- $\varnothing$ trigger efficiency as a function of instantaneous luminosity [103]. The fitted line is $\epsilon_{\mathrm{L} \varnothing}=$ $0.897+0.00124 \mathcal{L}$.

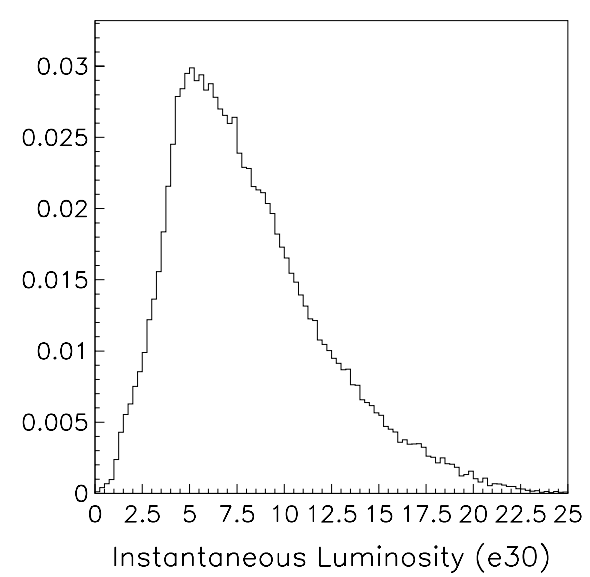

Figure 8.3: Distribution of the integrated luminosity of the entire data sample as a function of instantaneous luminosity [103].

\subsubsection{Correction for Multiple Single-Diffractive Interactions}

The Level- $\varnothing$ array acceptance for single-diffractive interactions, $\mathcal{A}_{S D}$, is determined by assuming that the same single-diffractive interaction is able to produce a 
charged particle hit in each of the two arrays. This acceptance is low because a diffraction particle is infrequently emitted in the direction opposite to that of the initial proton (or antiproton). Thus, most single-diffractive interactions are characterized by a charged particle hitting one array but not the other. In a high luminosity environment, it becomes possible to produce multiple single-diffractive interactions, where the diffraction occurs in opposite directions for the two (or more) simultaneous interactions. The situation becomes very similar to the double-diffractive case, where the acceptance is higher. These multiple single-diffractive interactions are included in the overall acceptance derived in Section 8.2.2, but a luminosity dependent weighting factor is still required to adjust the acceptance to the instantaneous luminosity profile of the data sample. This weighting (or correction) factor, $f_{M S D}$, as a function of instantaneous luminosity is shown in Figure 8.4. In addition, the average luminosity-weighted correction for all data recorded below the corresponding instantaneous luminosity is shown in Figure 8.5.

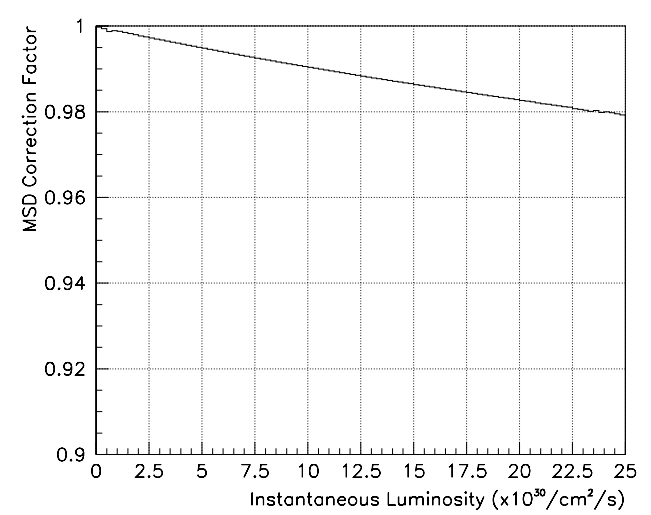

Figure 8.4: The average multiple single diffractive correction as a function of instantaneous luminosity [103].

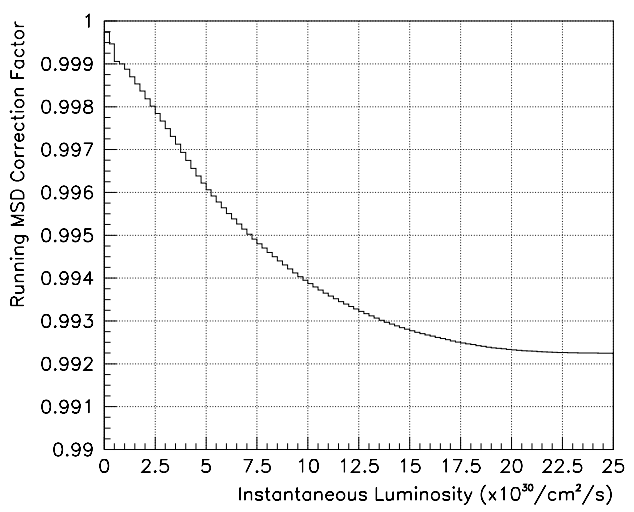

Figure 8.5: The running average multiple single diffractive correction as a function of instantaneous luminosity [103]. 


\subsubsection{Correction for Beam Halo}

Beam halo is another effect which becomes significant at higher luminosities, and thus introduces luminosity dependence which must be considered. Beam halo can veto a beam crossing, thereby lowering the luminosity. Although the accelerator operated at typically less than $1 \%$ halo ( $p$ and $\bar{p}$ combined), the effect at the higher luminosities was significant. Since the Level- $\varnothing$ fast $z$ calculation was an integral part of the physics triggers, no correction is needed at low luminosities, since a halo veto also vetoes the physics triggers. The halo factor, $f_{\text {halo }}$, is introduced to correct the error due to the multiple interaction correction portion of the halo term. The Level- $\varnothing$ slow $z$ calculation was unaffected by halo, and served as a baseline when determining the correction factor needed to remove halo effects. The dependence of the correction factor $f_{\text {halo }}$ on instantaneous luminosity is shown in Figure 8.6. In addition, the running of this correction factor for the integrated luminosity calculation for all data up to a given instantaneous luminosity is shown in Figure 8.7.

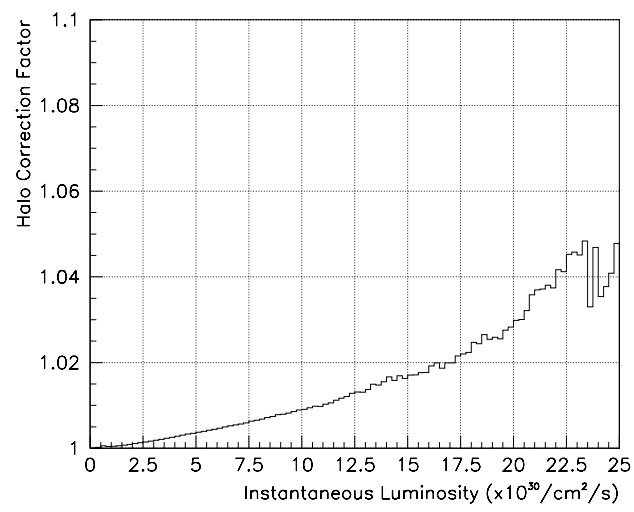

Figure 8.6: The beam halo correction as a function of instantaneous luminosity [103].

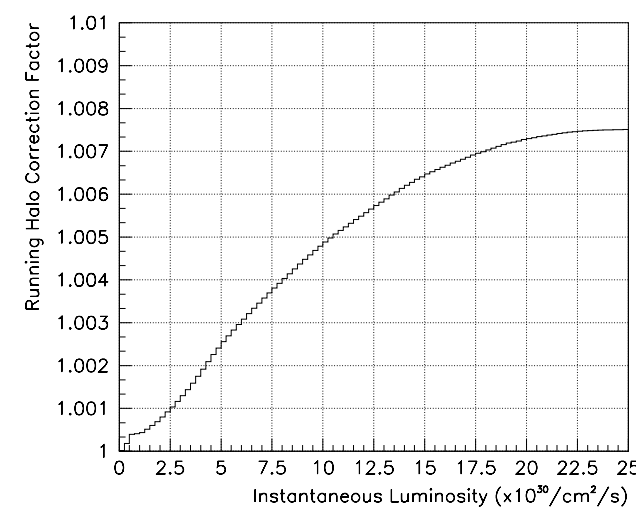

Figure 8.7: The running beam halo correction as a function of instantaneous luminosity [103]. 


\subsubsection{Calculation of $\sigma_{\mathrm{L} \varnothing}$}

Before performing the calculation of $\sigma_{\mathrm{L} \varnothing}$, it is interesting to note that the halo and multiple single-diffraction correction factors are roughly equal and opposite at most luminosities. Figure 8.8 shows the dependence of the average combined correction factor, $f_{\text {halo }} \times f_{M S D}$, on the instantaneous luminosity, where it is apparent that the beam halo correction is dominant at higher luminosities. The running combined correction factor for the integrated luminosity calculation for all data up to a given instantaneous luminosity is shown in Figure 8.9. The overall shift in the integrated luminosity for the entire data set is only $0.03 \%$. Even though this overall correction seems small, the correction for data obtained at the predominantly lower luminosities is larger.

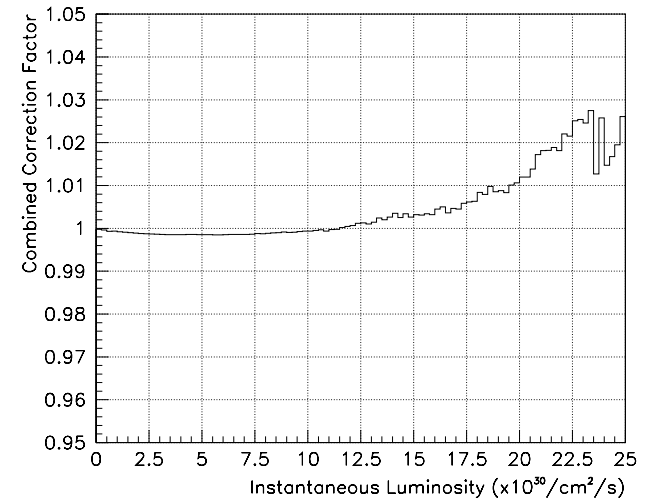

Figure 8.8: The combined beam halo and multiple single diffractive correction as a function of instantaneous luminosity [103].

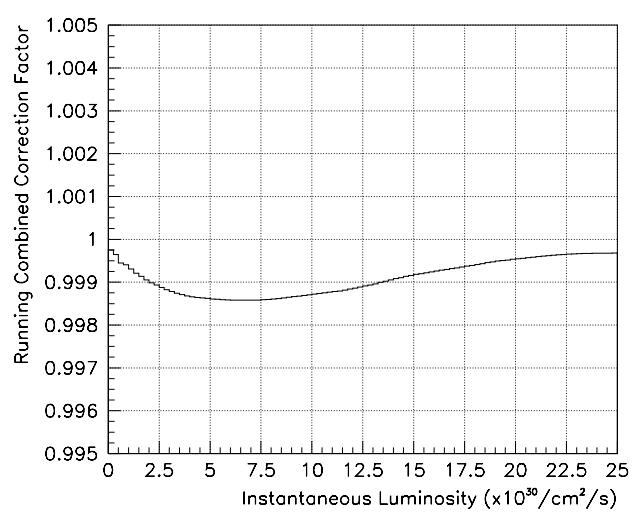

Figure 8.9: The running combined beam halo and multiple single diffractive correction as a function of instantaneous luminosity [103].

The calculation of $\sigma_{\mathrm{L} \varnothing}$ requires the values calculated in the previous sections as input to:

$$
\sigma_{\mathrm{L} \emptyset}=\epsilon_{\mathrm{L} \varnothing} f_{\text {halo }} f_{M S D} \sum_{i} \mathcal{A}_{i} \sigma_{i} \quad(i=S D, D D, H C)
$$


The results of the calculation expressed in Equation 8.14 are summarized in Table 8.4 where the luminosity weighted Level- $\varnothing$ efficiency, $\epsilon_{\mathrm{L} \varnothing}$, and combined halo and MSD correction factors, $f_{\text {halo }} \times f_{M S D}$, are used to determine the luminosity weighted Level- $\varnothing$ monitor constant $\sigma_{\mathrm{L} \varnothing}$.

Table 8.4: Results of the calculation of the luminosity weighted average $\sigma_{\mathrm{L} \emptyset}$.

\begin{tabular}{ccc}
\hline \hline & Mean & Error \\
\hline $\mathcal{A}_{S D} \sigma_{S D}+\mathcal{A}_{D D} \sigma_{D D}+\mathcal{A}_{H C} \sigma_{H}$ & $49.11 \mathrm{mb}$ & $2.45 \mathrm{mb}$ \\
$\epsilon_{\mathrm{L} \emptyset}$ & 0.907 & 0.017 \\
$f_{\text {halo }} \times f_{M S D}$ & 0.99975 & 0.0020 \\
\hline$\sigma_{\mathrm{L} \emptyset}$ & $44.53 \mathrm{mb}$ & $2.37 \mathrm{mb}$ \\
\hline \hline
\end{tabular}

\subsection{Determination of Integrated Luminosity}

In order to determine the total luminosity used in the analysis, the instantaneous luminosity $\mathcal{L}$ is integrated over the live running time used in collecting the data. The information needed to calculate $\mathcal{L}$ is stored periodically where the period is chosen to be small enough so that $\mathcal{L}$ does not vary appreciably, thus minimizing the inaccuracies induced by the numerical integration method. In addition, knowledge of any deadtime in the data acquisition system must be recorded since it affects the calculation of the integrated luminosity. There are many sources of dead-time for any trigger, such as a front-end busy (this occurs when the front-end digitizing crates are not ready to process data), Level-2 busy (when there are no Level-2 nodes available to process an event), Main Ring vetoes, or a prescale. In general, a run has several different 
triggers, each having a different live-time. Enough data must be stored in order to calculate each trigger live-time separately.

The information regarding luminosity is handled by the Luminosity Server, a program that runs in the background of the online computer system. It accesses the Level-1 scaler information, along with relevant information from the accelerator and the data acquisition system, once per minute, and writes the current values of this information to a database once every ten minutes. This time scale is short compared to the typical lifetime of a store ( 24 hours $)$. All necessary information to calculate the integrated luminosity for a specific trigger is contained in the database.

\subsubsection{Luminosity Integral}

The instantaneous luminosity is determined by looking at the firing rate of the Level- $\varnothing$ system. As was discussed previously, the equations relating the instantaneous luminosity to this firing rate become non-linear in the high luminosity regime. On the other hand, the luminosity is slowly varying with a time constant of the order of 24 hours. Integrating over small enough time periods (10 minutes) allows one to evaluate the integrated luminosity knowing only the live time fraction. The integration, then, becomes a simple sum [111]:

$$
\int \mathcal{L}(T) d t=\sum_{b, i} \overline{\mathcal{L}}(b, i) \cdot f_{\text {live }}(i, b, T) \cdot \Delta t(i)
$$


where $\overline{\mathcal{L}}(b, i)$ is the average instantaneous luminosity for the bunch-pair $b$ in the time period $i, f_{\text {live }}(i, b, T)$ is the corresponding live-time for a specific trigger $T$, and $\Delta t(i)$ is the time interval between two successive readings $i$ and $i+1$.

The luminosity integral proposed in Equation 8.15 reflects quite a bit of generality, namely that each Level-1 trigger has an associated Level-1 scaler from which the live-time can be measured. This amounts to a total of $6 \times 32$ scalers for each Nbunch $\times$ Nbit. Unfortunately, this total is larger than the available number of scalers. Furthermore, since the Level-1 trigger conditions are defined at run time, each scaler would require fully programmable logic hardware, as flexible as the decision hardware itself. The Level-1 framework does not offer such flexibility.

The solution to this dilemma is to impose the requirement that all sources of non-physics dead-time be common to all of the Level-1 triggers. With this requirement imposed, it is possible to dedicate one particular trigger bit (bit 30) to keeping track of the live-time. The signal from bit 30 ANDed with the output of the Level- $\varnothing$ system measures live luminous crossings containing one or more interactions. The integrated luminosity for any given time period is then obtained by dividing the number of luminous crossings by the Level- $\varnothing$ luminosity constant $\sigma_{\mathrm{L} \varnothing}$. The correction for multiple interactions, as expressed in Equation 8.7, must still be applied.

The requirement that all sources of non-physics dead-time be common to all Level-1 triggers implies the following conditions: 
- All Level-2 nodes must be available for all Level-1 triggers, with a single class of nodes treating all physics triggers (i.e. one cannot dedicate a certain class of nodes for more important triggers);

- All front-end busy signals affect all Level-1 triggers the same, so that all physics triggers read out the same crates (one cannot read out a subset of the front-end crates for some triggers in an attempt to reduce the bandwidth);

- The Level-1 trigger bit 30 is set up so that it is not prescaled, contains no physics conditions, and never generates an actual Level-1 trigger. It must, however, share all non-physics conditions used by the physics triggers and observe those and only those sources of dead-time observed by the physics triggers.

As a result, all required load balancing in the data acquisition system is done by adjusting the various thresholds, or by using fixed prescales. Scalers in the Level-1 framework are assigned to each trigger bit and count the number of events that pass after the prescale is applied. The prescale is assumed to be bunch independent, thus one scaler is required for each trigger instead of six. In addition, separate scalers are needed to measure the dead time introduced by each Main Ring veto (such as MRBS_LOSS Or MICRO_BLANK).

For a given specific trigger $T$, the luminosity integral of Equation 8.15 is rewritten as $[106$, p. 145]:

$$
\int \mathcal{L} d t=\left(\sum_{i, b} \frac{N_{\mathrm{L} \varnothing}^{\text {live }}(i, b)}{\sigma_{\mathrm{L} \varnothing}} \cdot M(i, b) \cdot N P(i, b, T)\right) \cdot P(T)
$$


where $b=(1, \ldots, 6)$ is the bunch pair index, $i$ is the time period index, $N_{\mathrm{L} \varnothing}^{\text {live }}$ is the total number of live crossings as measured by the Level- $\varnothing$ system, $\sigma_{\mathrm{L} \varnothing}$ is the luminosity monitor constant, $M(i, b)$ is the luminosity dependent correction factor (multiple interaction, beam halo, multiple single diffractive, and Level- $\varnothing$ efficiency corrections), $N P(i, b, T)$ is the correction for non-physics conditions (such as Main Ring veto or front-end busy), and $P(T)$ is the prescale correction at Level-1 and Level-2.

\subsection{Offline Luminosity Bookkeeping}

The luminosity database created by the Luminosity Server contains all the available information needed to compute the integrated luminosity for each run. This database is not very practical for physics analysis, since what is required is the integrated luminosity of a particular data file (STA, DST, or $\mu \mathrm{DST}$ ). In order to meet this demand for each analysis effort, the information in the luminosity database is processed, and the resultant luminosity corresponding to the raw data files are stored in the production database. For any given run, the production database contains information describing the luminosity (and any applied corrections) for each trigger.

The data for each run are split into several files, or partitions ${ }^{2}$, making it easier to handle and process. The integrated luminosity for each RAW partition is made

\footnotetext{
${ }^{2}$ The maximum number of partitions for any given run is 99 .
} 
proportional to the number of events in the partition:

$$
\mathcal{L}_{R A W}=\mathcal{L}_{R U N} \cdot \frac{n_{R A W}}{n_{R U N}}
$$

During the offline reconstruction process and the subsequent streaming and filtering of the data, events as well as entire partitions can be lost (perhaps due to a crash in DØRECO, or a failure in reading the $8 \mathrm{~mm}$ Exabyte tapes). The production database utilities are able to correct for this effect, by comparing the number of events contained in the output files (STA, DST, or $\mu \mathrm{DST}$ ) to the number of events in the corresponding RAW file. The luminosities of each output file is calculated as:

$$
\mathcal{L}_{i}=\mathcal{L}_{R A W} \cdot \frac{n_{i}}{n_{R A W}} \quad(i=\mathrm{STA}, \mathrm{DST} \text { or } \mu \mathrm{DST})
$$

\subsection{Integrated Luminosity of the Data Samples}

The $W$ and $Z$ event samples were selected from the $\mu$ DST data files residing on the $\mathrm{D} \varnothing$ file server. Routines were written to filter $W$ and $Z$ events by hand [112] with very loose cuts $^{3}$. The resultant output files were made available to the D $\varnothing$ Electroweak working group by placing them on dedicated 9 Gbyte hard disks. The entire analysis was performed on these filtered files.

The selection of $W$ and $Z$ events, outlined in Chapter 4, is based on two separate triggers. The $Z$ trigger, EM2_EIS2_HI, was never prescaled during the entire data taking period. The $W$ trigger, EM1_EISTRKCC_MS, was for the most part unprescaled,

\footnotetext{
${ }^{3}$ The $W \rightarrow e \nu$ filter cuts required one EM object with $E_{T}>15 \mathrm{GeV}$ and $\not_{T}>15 \mathrm{Gev}$; the $Z \rightarrow e e$ filter cuts required two EM objets with $E_{T}>10 \mathrm{GeV}$ each. No electron identification criteria was applied.
} 
although there were a dozen runs where a prescale was applied (this was during very high luminosity running before the introduction of the Level-1.5 Calorimeter trigger). In addition, the offline selection criteria for $W \rightarrow e \nu$ events requires the GoODBEAM Main Ring veto, which is different than the GoodCAL veto applied to the trigger. To calculate the luminosity for that particular veto, the integrated luminosity of the MISSING_ET trigger is requested. The MISSING_ET trigger operated with the GoodBeAm condition applied, and was never prescaled during Run Ib. A correction is made to account for those runs when the $W \rightarrow e \nu$ trigger was prescaled. The luminosity results for the $W$ and $Z$ triggers are summarized in Table 8.5. The error on the results is solely due to the $5.3 \%$ error on the Level- $\varnothing$ luminosity constant $\sigma_{\mathrm{L} \varnothing}$; the error due to bookkeeping is negligible in comparison. In addition, by taking the ratio of the luminosities of the $W$ to the $Z$ samples, all the errors completely cancel.

Table 8.5: Integrated luminosity results for the $W \rightarrow e \nu$ and $Z \rightarrow e e$ data samples.

\begin{tabular}{lcc}
\hline \hline & EM1_EISTRKCC_MS & EM2_EIS2_HI \\
\hline Corrected Luminosity & $82.4 \mathrm{pb}^{-1}$ & $108.5 \mathrm{pb}^{-1}$ \\
Error & $\pm 4.4 \mathrm{pb}^{-1}$ & $\pm 5.8 \mathrm{pb}^{-1}$ \\
\hline \hline
\end{tabular}




\section{Chapter 9}

\section{Results and Conclusion}

At this juncture, all the relevant parameters for the calculation of the production cross sections times electronic branching ratio of $W$ and $Z$ bosons have been measured. Hence the results are presented, along with the extraction of the electronic branching fraction of the $W$ boson, $\operatorname{Br}(W \rightarrow e \nu)$, and its total width, , $W$. The future prospects of similar measurements are also discussed.

\subsection{Cross Section Calculation for $W$ Production}

The cross section times electronic branching ratio for $W$ boson production is computed from:

$$
\sigma \cdot \operatorname{Br}(p \bar{p} \rightarrow W \rightarrow e \nu)=\frac{N_{s i g}^{w} \cdot\left(1-f_{b k g}^{w}\right)}{\mathcal{A}_{w} \cdot \varepsilon_{w} \cdot \mathcal{L}_{i n t}^{w}}
$$

where $N_{s i g}^{w}$ is the number of candidate $W \rightarrow e \nu$ events, $f_{b k g}^{w}$ is the background fraction, and $\mathcal{A}_{w}, \varepsilon_{w}$, and $\mathcal{L}_{\text {int }}^{w}$ are the acceptance, overall efficiency, and integrated luminosity, respectively. These input parameters are summarized in Table 9.1. It is important to note that the quoted background fractions due to $W \rightarrow \tau \nu$ and $Z \rightarrow e e$ decays have 
Table 9.1: Summary of input parameters to the $W \rightarrow e \nu$ cross section results.

\begin{tabular}{lccc}
\hline \hline & CC & EC & Total \\
\hline Events & 47004 & 20402 & 67406 \\
\hline Efficiency (\%) & $70.10 \pm 0.91$ & $62.27 \pm 0.95$ & $67.75 \pm 1.04$ \\
\hline Acceptance (\%) & $32.84 \pm 0.26$ & $14.30 \pm 0.11$ & $47.14 \pm 0.38$ \\
\hline Total Bckg (\%) & $6.62 \pm 0.75$ & $15.65 \pm 1.10$ & $9.35 \pm 0.84$ \\
QCD (\%) & $3.96 \pm 0.72$ & $13.27 \pm 1.07$ & $6.78 \pm 0.81$ \\
$W \rightarrow \tau \nu(\%)$ & $1.97 \pm 0.21$ & $1.74 \pm 0.21$ & $1.90 \pm 0.21$ \\
$Z \rightarrow e e, Z \rightarrow \tau \tau(\%)$ & $0.70 \pm 0.06$ & $0.63 \pm 0.05$ & $0.68 \pm 0.06$ \\
\hline $\mathcal{L}_{\text {int }}^{w}\left(\mathrm{pb}^{-1}\right)$ & $82.4 \pm 4.4$ & $82.4 \pm 4.4$ & $82.4 \pm 4.4$ \\
\hline \hline
\end{tabular}

been converted to fractions of the number of observed events:

$$
\begin{aligned}
f_{\tau}^{w^{\prime}} & \rightarrow f_{\tau}^{w} \cdot \frac{1-f_{q c d}^{w}}{1+f_{\tau}^{w}+f_{z}^{w}} \\
f_{z}^{w^{\prime}} & \rightarrow f_{z}^{w} \cdot \frac{1-f_{q c d}^{w}}{1+f_{\tau}^{w}+f_{z}^{w}}
\end{aligned}
$$

where $f_{\mathcal{\tau}}^{w^{\prime}}$ and $f_{z}^{w^{\prime}}$ are the values listed in Table 9.1, and $f_{\tau}^{w}$ and $f_{z}^{w}$ the values computed in Chapter 7.

After inserting the input parameters into Equation 9.1, the total cross section times branching ratio is measured to be:

$$
\sigma \cdot \operatorname{Br}(p \bar{p} \rightarrow W \rightarrow e \nu)=2.322 \pm 0.009 \text { (stat) } \pm 0.046 \text { (syst) } \pm 0.123 \text { (lum) nb. }
$$

The error due to the luminosity uncertainty has been singled out, since it is clearly dominant. It is interesting to note that even when ignoring this luminosity error, this measurement is still dominated by the systematic uncertainty. However, a large portion of this systematic error is due to the efficiency calculation, which is itself limited by the number of available $Z$ events. 
As a consistency check, the cross sections have also been measured separately for $\mathrm{CC}$ and EC events:

$$
\begin{aligned}
& \mathrm{CC}: \sigma \cdot \operatorname{Br}(p \bar{p} \rightarrow W \rightarrow e \nu)=2.314 \pm 0.011 \pm 0.040 \mathrm{nb} \\
& \mathrm{EC}: \sigma \cdot \operatorname{Br}(p \bar{p} \rightarrow W \rightarrow e \nu)=2.347 \pm 0.016 \pm 0.051 \mathrm{nb}
\end{aligned}
$$

where the common luminosity error has been ignored. The agreement is quite good, adding confidence to the overall measurement.

\subsection{Cross Section Calculation for $Z$ Production}

The cross section times electronic branching ratio for $Z$ boson production is computed from:

$$
\sigma \cdot \operatorname{Br}(p \bar{p} \rightarrow Z \rightarrow e e)=\frac{N_{s i g}^{z} \cdot\left(1-f_{b k g}^{z}\right)}{\mathcal{A}_{z} \cdot \varepsilon_{z} \cdot \mathcal{L}_{i n t}^{z}}
$$

where $N_{\text {sig }}^{z}$ is the number of candidate $Z \rightarrow e e$ events, $f_{b k g}^{z}$ is the background fraction, and $\mathcal{A}_{z}, \varepsilon_{z}$, and $\mathcal{L}_{\text {int }}^{z}$ are the acceptance, overall efficiency, and integrated luminosity, respectively. These input parameters are summarized in Table 9.2. The quoted background fraction due to Drell-Yan has been converted to a fraction of the number of observed $Z$ candidates:

$$
f_{d-y}^{z^{\prime}} \rightarrow f_{d-y}^{z} \cdot \frac{1-f_{q c d}^{z}}{1+f_{d-y}^{z}}
$$

where $f_{d-y}^{z^{\prime}}$ is the value listed in Table 9.2 , while $f_{d-y}^{z}$ is the value computed in Chapter 7. 
Table 9.2: Summary of input parameters to the $Z \rightarrow e e$ cross section results.

\begin{tabular}{lcccc}
\hline \hline & $\mathrm{CC} / \mathrm{CC}$ & $\mathrm{CC} / \mathrm{EC}$ & $\mathrm{EC} / \mathrm{EC}$ & Total \\
\hline Events & 3628 & 2831 & 681 & 7140 \\
\hline Efficiency (\%) & $78.32 \pm 1.14$ & $73.53 \pm 0.90$ & $68.83 \pm 1.07$ & $75.43 \pm 0.94$ \\
\hline Acceptance (\%) & $18.57 \pm 0.15$ & $15.11 \pm 0.13$ & $3.76 \pm 0.03$ & $37.44 \pm 0.31$ \\
\hline Total Bckg (\%) & $3.10 \pm 0.44$ & $7.61 \pm 0.91$ & $6.65 \pm 0.98$ & $5.23 \pm 0.45$ \\
$\mathrm{QCD}(\%)$ & $2.11 \pm 0.43$ & $6.59 \pm 0.90$ & $4.78 \pm 0.96$ & $4.14 \pm 0.43$ \\
$Z / \gamma^{\star}(\%)$ & $0.99 \pm 0.10$ & $1.02 \pm 0.11$ & $1.87 \pm 0.20$ & $1.90 \pm 0.12$ \\
\hline $\mathcal{L}_{\text {int }}^{z}\left(\mathrm{pb}^{-1}\right)$ & $108.5 \pm 5.7$ & $108.5 \pm 5.7$ & $108.5 \pm 5.7$ & $108.5 \pm 5.7$ \\
\hline \hline
\end{tabular}

After inserting the input parameters into Equation 9.7, the total cross section times branching ratio is measured to be:

$$
\sigma \cdot \operatorname{Br}(p \bar{p} \rightarrow Z \rightarrow e e)=0.220 \pm 0.003 \text { (stat) } \pm 0.003 \text { (syst) } \pm 0.012 \text { (lum) nb. }
$$

The error due to the luminosity uncertainty is dominant in this result as well, and consequently has been singled out. In contrast to the $W$ boson result, the statistical and systematic errors are much closer. This is due to the limited statistics in the signal sample.

As a consistency check, the cross sections have also been measured separately for each cryostat combination:

$$
\begin{aligned}
& \mathrm{CC}-\mathrm{CC}: \sigma \cdot \operatorname{Br}(p \bar{p} \rightarrow Z \rightarrow e e)=0.223 \pm 0.004 \pm 0.004 \mathrm{nb} \\
& \mathrm{CC}-\mathrm{EC}: \sigma \cdot \operatorname{Br}(p \bar{p} \rightarrow Z \rightarrow e e)=0.217 \pm 0.004 \pm 0.004 \mathrm{nb} \\
& \mathrm{EC}-\mathrm{EC}: \sigma \cdot \operatorname{Br}(p \bar{p} \rightarrow Z \rightarrow e e)=0.226 \pm 0.009 \pm 0.005 \mathrm{nb}
\end{aligned}
$$

where the common luminosity error has been ignored. In this case, the agreement is quite good as well. 


\subsection{The Ratio $R$ of Cross Sections}

The ratio of $W$ and $Z$ cross sections is computed from:

$$
R=\frac{\sigma_{W} \cdot \operatorname{Br}(W \rightarrow e \nu)}{\sigma_{Z} \cdot \operatorname{Br}(Z \rightarrow e e)}=\frac{N_{w}}{N_{z}} \cdot \frac{\mathcal{A}_{z}}{\mathcal{A}_{w}} \cdot \frac{\varepsilon_{z}}{\varepsilon_{w}} \cdot \frac{\mathcal{L}_{i n t}^{z}}{\mathcal{L}_{i n t}^{w}}
$$

where $N_{w}$ and $N_{z}$ are the estimated number of true $W$ and $Z$ bosons, after background subtraction. Many sources of systematic errors cancel in the ratio of the cross sections since many of the uncertainties are correlated. The uncertainty in the luminosity calculation is assumed to cancel completely in the ratio. A simple Monte Carlo is written to determine the overall uncertainty in the cross section ratio due to electron efficiency uncertainties (see Section 6.4.3). The uncertainty in the acceptance ratio was determined directly by calculating the variance in the ratio of the acceptances for various input uncertainties (see Section 5.3.1). The values necessary to calculate $R$ are summarized in Table 9.3. The final cross section ratio is measured to be:

$$
R=10.51 \pm 0.13 \text { (stat) } \pm 0.14 \text { (syst) }
$$

Added in quadrature, this results in a fractional error of $1.8 \%$.

Table 9.3: Summary of input parameters to the cross section ratio $R$.

\begin{tabular}{cc}
\hline \hline Input & Value \\
\hline$N_{w}$ & $61103 \pm 260 \pm 567$ \\
$N_{z}$ & $6767 \pm 84 \pm 32$ \\
$\mathcal{A}_{z} / \mathcal{A}_{w}$ & $0.794 \pm 0.005$ \\
$\varepsilon_{z} / \varepsilon_{w}$ & $1.113 \pm 0.005$ \\
$\mathcal{L}_{\text {int }}^{z} / \mathcal{L}_{\text {int }}^{w}$ & 1.316 \\
\hline \hline
\end{tabular}




\subsection{Extraction of the $W$ Width}

From the definition of the cross section ratio

$$
R=\frac{\sigma_{W}}{\sigma_{Z}} \cdot \frac{\operatorname{Br}(W \rightarrow e \nu)}{\operatorname{Br}(Z \rightarrow e e)}
$$

one can indirectly obtain the electronic branching fraction of the $W$ boson:

$$
\operatorname{Br}(W \rightarrow e \nu)=R \cdot \operatorname{Br}(Z \rightarrow e e) \cdot \frac{\sigma_{Z}}{\sigma_{W}}
$$

Using the measured value of the ratio $R$ and assuming standard model couplings, $\operatorname{Br}(W \rightarrow e \nu)$ is measured to be:

$$
\operatorname{Br}(W \rightarrow e \nu)=(10.75 \pm 0.13(\text { stat }) \pm 0.14(\text { syst }) \pm 0.09(\text { external })) \%
$$

The last error arises from measurements of $\operatorname{Br}(Z \rightarrow e e)$ at LEP, $(3.367 \pm 0.006) \%$ [18], and the predicted ratio of total cross, $\sigma_{W} / \sigma_{Z}=3.29 \pm 0.03[6]$.

By further assuming the standard model partial width, $(W \rightarrow e \nu)$, calculated to be $0.2270 \pm 0.0011 \mathrm{GeV}[10]$, one obtains the total decay width of the $W$ as

$$
{ }_{W}=2.11 \pm 0.026(\text { stat }) \pm 0.028(\text { syst }) \pm 0.013(\text { external }) \mathrm{GeV}
$$

Added in quadrature, this results in a fractional uncertainty of $1.9 \%$.

\subsection{Conclusions}

The focus of this dissertation was the measurement of the ratio of production cross section of electronically decaying $W$ and $Z$ bosons. From a sample of 67406 
$W \rightarrow e \nu$ and $7140 Z \rightarrow e e$ events found in $82.4 \mathrm{pb}^{-1}$ and $108.5 \mathrm{pb}^{-1}$ of data respectively, the inclusive cross sections times branching fractions, and their ratio, are measured to be:

$$
\begin{aligned}
\sigma_{W} \cdot \operatorname{Br}(W \rightarrow e \nu) & =2.322 \pm 0.009 \pm 0.046 \pm 0.123 \mathrm{nb} \\
\sigma_{Z} \cdot \operatorname{Br}(Z \rightarrow e e) & =0.221 \pm 0.003 \pm 0.003 \pm 0.012 \mathrm{nb} \\
R & =10.51 \pm 0.13 \pm 0.14
\end{aligned}
$$

The quoted errors on the cross sections are statistical, systematic, and luminosity errors respectively. The quoted errors on the ratio are statistical and systematic. These three measurements are in good agreement with the Standard Model theoretical predictions $^{1}$ :

$$
\begin{aligned}
\sigma_{W} \cdot \operatorname{Br}(W \rightarrow \ell \nu) & =2.414 \pm 0.095 \mathrm{nb} \\
\sigma_{Z} \cdot \operatorname{Br}(Z \rightarrow \ell \ell) & =0.228 \pm 0.008 \mathrm{nb} \\
R & =10.59 \pm 0.09
\end{aligned}
$$

From the measured value of $R$ and Standard Model inputs, the branching fraction $\operatorname{Br}(W \rightarrow e \nu)$, and the $W$ total width , $W$, are extracted:

$$
\begin{array}{r}
\operatorname{Br}(W \rightarrow e \nu)=(10.75 \pm 0.21) \% \\
, W=2.12 \pm 0.040 \mathrm{GeV} .
\end{array}
$$

They are in agreement with the Standard Model theoretical predictions:

$$
\begin{array}{r}
\operatorname{Br}(W \rightarrow e \nu)=(10.84 \pm 0.02) \% \\
, W=2.094 \pm 0.009 \mathrm{GeV} .
\end{array}
$$

\footnotetext{
${ }^{1}$ The errors are due to the choice of structure functions, and the error on the $W$ boson mass.
} 
Using the measured value of,$w$ and its Standard Model prediction, one can set an upper limit on the contribution of unexpected decays to the $W$ width. At $95 \%$ confidence level, the limit is $93 \mathrm{MeV}$.

\subsection{Future Prospects}

The $W$ boson width, , $W$, has been measured in this dissertation to $1.9 \%$, yielding an error of $40 \mathrm{MeV}$. A further reduction in error is possible by combining this result with the one from the $\mathrm{CDF}$ experiment (once that analysis is finalized), to obtain a combined Tevatron result. Even though both experiments are pursuing a direct measurement from a fit to the $W$ transverse mass tail, the expected errors of $\sim 150 \mathrm{MeV}$ will not be competitive. At the present moment, the only remaining measurement will be made in electron-positron collisions at LEP2, where $\sim 300 \mathrm{MeV}$ precision is expected to be achieved [113].

Preparations are under way for the Tevatron collider Run II, scheduled to begin in 1999 and accumulate $\sim 1 \mathrm{fb}^{-1}$ of data. With this very large sample, the expected error on ${ }_{W}$ from the ratio measurement is expected to be reduced to $\sim 30 \mathrm{MeV}$. This expected error will be dominated by the theoretical uncertainties in the calculation of $\sigma_{W} / \sigma_{Z}$. The direct measurement of, ${ }_{W}$ from a fit to the tail of the $W$ transverse mass distribution is expected to yield an error of $\sim 50 \mathrm{MeV}$. This measurement will be statistically limited. However, there are discussions about upgrading the Tevatron to provide even higher luminosities and energies after Run II. With this scenario, 
an accumulated data set of $10 \mathrm{fb}^{-1}$ can yield an error of $\sim 20 \mathrm{MeV}$ from either technique [114]. The combined results from the electron and muon channels, and measurements from CDF and DØ, should reach an overall accuracy of $10 \mathrm{MeV}$, thus approaching the level of the electroweak corrections to , ${ }_{W}[10]$. 


\section{Bibliography}

[1] E. Abers and B. W. Lee, Phys. Rep. C9, 1 (1973).

C. N. Yang and T. D. Lee, Phys. Rev. 96, 191 (1954).

[2] S. Glashow, Nucl. Phys. 22, 579 (1961).

S. Weinberg, Phys. Lett. 12, 132 (1967).

A. Salam, Elementary Particle Physics, N. Svartholm ed., 367 (1968).

[3] H. Fritzsch and M. Gell-Mann, in Proceedings of the Sixteenth International Conference on High Energy Physics, Chicago, 1972, vol. 2, p. 135.

S. Weinberg, Phys. Rev. Lett. 31, 494 (1973).

D. J. Gross and F. Wilczek, Phys. Rev. D8, 3633 (1973).

H. Fritzsch, M. Gell-Mann and H. Leutwyler, Phys. Lett. 47B, 365 (1973).

[4] S. D. Drell and T. M. Yan, Phys. Rev. Lett. 25, 316 (1970).

S. D. Drell and T. M. Yan, Ann Phys. 66, 578 (1971).

[5] V. D. Barger and R. J. N. Phillips, Collider Physics. Addision-Wesley Publishing Company (1987).

[6] R. Hamberg, W. L. van Neerven and T. Matsuura, Nucl. Phys. B359, 343 (1991).

W. L. van Neerven and E. B. Zijlstra, Nucl. Phys. B382, 11 (1992).

[7] E. L. Berger et al., Phys. Rev. D40, 83 (1989).

[8] H. Plothow-Besch, PDFLIB: Nucleon, Pion and Photon Parton Density Functions and $\alpha_{S}$ Calculations User's Manual, CERN Program Library Entry W0588 (1997).

[9] A. Sirlin, Phys. Rev. D22, 971 (1980).

W. Hollick, Fortschr. Phys. 38, 165 (1990).

[10] J. L. Rosner, M. P. Worah and T. Takeushi, Phys. Rev. D49, 1363 (1994).

[11] A. Denner and T. Sack, Zeit. Phys. C46, 653 (1990).

[12] V. Barger et al., Phys. Rev. D28, 2912 (1983).

M. Drees, C. S. Kim and X. Tata, Phys. Rev. D37, 784 (1988).

[13] T. Alvarez, A. Leites and J. Terrón, Nucl. Phys. B301, 1 (1988). 
[14] J. Smith, W. L. vanNeerven, and J. A. M. Vermaseren, Phys. Rev. Lett. 50, 1738 (1983).

[15] N. Cabibbo, What Next? in Proceedings of the 3rd Workshop on p $\bar{p}$ Collider Physics, Rome, Italy, (1983).

[16] G. Arnison et al., Phys. Lett. B112, 103 (1983).

G. Arnison et al., Phys. Lett. B126, 398 (1983).

G. Arnison et al., Phys. Lett. B129, 273 (1983).

G. Arnison et al., Phys. Lett. B134, 469 (1983).

[17] M. Banner et al., Phys. Lett. B122, 476 (1983).

P. Bagnaia et al., Phys. Lett. B129, 130 (1983).

[18] Particle Data Group, L. Montanet et al., Phys. Rev. D54, 1 (1996).

[19] C. Albajar et al., Zeit. Phys. C44, 15 (1989).

[20] J. Alitti et al., Zeit. Phys. C47, 11 (1990).

[21] F. Abe et al., Phys. Rev. Lett. 64, 152 (1990).

[22] F. Abe et al., Phys. Rev. Lett. 69, 28 (1992).

[23] C. Albajar et al., Phys. Lett. B253, 503 (1991).

[24] J. Alitti et al., Phys. Lett. B276, 365 (1992).

[25] F. Abe et al., Phys. Rev. D52, 2624 (1995).

[26] F. Abe et al., Phys. Rev. Lett. 74, 341 (1995).

[27] S. Abachi et al., Phys. Rev. Lett. 75, 1456 (1995).

[28] S. Abachi et al., Nucl. Instr. Meth. A338, 185 (1994).

[29] L. M. Lederman, Scientific American 264(3) 48 (Mar. 1991).

H. T. Edwards, Ann. Rev. Nucl. Part. Sci. 35, 605 (1985).

F. T. Cole et al., "Design Report Tevatron 1 Project," FNAL Internal Note (1984), unpublished.

F. T. Cole et al., "A Report of the Design of the Fermi National Laboratory Superconducting Accelerator," FNAL Internal Note (1979), unpublished.

[30] W. J. Thompson, "Introduction to Colliding Beams at Fermilab," DØ Internal Note 2367 (1994), unpublished.

[31] D. Möhl et al., Phys. Rep. C58, 73 (1980).

[32] B. Piffer et al., "An Experiment at DØ to Study Antiproton-Proton Collisions at $2 \mathrm{TeV}, "$ FNAL Internal Note (1983), unpublished.

[33] H. Williams, Ann. Rev. Nucl. Part. Sci. 36, 366 (1986). 
[34] F. Sauli, Principles of Operation of Multiwire Proportional and Drift Chambers in T. Ferbel, editor, Experimental Techniques in High Energy Physics. Addison-Wesley (1987).

[35] R. C. Fernow, Introduction to Experimental Particle Physics. Cambridge University Press (1986).

[36] K. Kleinknecht, Detectors for Particle Radiation. Cambridge University Press (1987).

[37] A. R. Clark et al., Nucl. Instr. Meth. A261, 420 (1987).

[38] A. R. Clark et al., Nucl. Instr. Meth. A279, 243 (1989).

[39] A. R. Clark et al., Nucl. Instr. Meth. A315, 193 (1992).

[40] J. F. Detœuf et al., Nucl. Instr. Meth. A265, 157 (1988).

[41] J. F. Detœuf et al., Nucl. Instr. Meth. A279, 310 (1989).

[42] D. Buchholz et al., Nucl. Instr. Meth. A257, 556 (1987).

[43] T. Behnke, The Central Drift Chamber for the DØ Detector: Design, Construction, and Test. Ph.D. thesis, State University of New York at Stony Broook, Stony Brook, New York, 1989 (unpublished).

[44] D. Pizzuto, DØ Central Tracking Performance Studies. Ph.D. thesis, State University of New York at Stony Broook, Stony Brook, New York, 1991 (unpublished).

[45] S. Rajagopalan, The $d E / d x$ Capabilities of the DØ Tracking System. Ph.D. thesis, Northwestern University, Evanston, Illinois, 1992 (unpublished).

[46] J. W. Bantly, The DØ Forward Drift Chamber Performance and Physics Capability in the 1990 FNAL Testbeam Run. Ph.D. thesis, Northwestern University, Evanston, Illinois, 1992 (unpublished).

[47] C. Fabjan, Calorimetry in High-Energy Physics in T. Ferbel, editor, Experimental Techniques in High Energy Physics. Addison-Wesley (1987).

[48] U. Amaldi, Fluctuations in Calorimetry Measurements in T. Ferbel, editor, Experimental Techniques in High Energy Physics. Addison-Wesley (1987).

[49] J. Kotcher, Response of the DØ Calorimeter to Cosmic Ray Muons. Ph.D. thesis, New York University, New York, 1992 (unpublished).

[50] C. Brown et al., Nucl. Instr. Meth. A279, 121 (1989).

[51] A. Lankford, Trigger and Data Acquisition at High Rate Colliders in Proceedings of the 20th SLAC Summer Institute, SLAC, Stanford, California (1992). 
[52] M. Breidenbach, Data Acquisition for High Energy Physics Experiments in Proceedings of the 14th SLAC Summer Institute, SLAC, Stanford, California (1986).

[53] R. K. Bock, H. Grote, D. Notz, and M. Regler, Data Analysis Techniques for High Energy Physics Experiments. Cambridge University Press (1990).

[54] J. Bantly et al., IEEE Trans. Nucl. Sci. NS-41, 1274 (1994).

[55] M. Abolins et al., IEEE Trans. Nucl. Sci. NS-36, 384 (1989).

[56] M. Abolins et al., Nucl. Instr. Meth. A289, 543 (1990).

[57] J. Drinkard, "The D $\varnothing$ Level 1.5 Calorimeter Trigger," DØ Internal Note 2274 (1994), unpublished.

[58] R. Genik, A DSP Based Electromagnetic Trigger for the D $\emptyset$ Detector in Proceedings of the 1995 IEEE Conference on Real Time Computer Applications in Nuclear, Particle, and Plasma Physics, East Lansing, Michigan (1995).

[59] D. Cutts et al., IEEE Trans. Nucl. Sci. NS-36, 738 (1989).

[60] D. Cutts et al., Operation of the DØ Data Acquisition System in Proceedings of the Conference on Computing in High Energy Physics, Annecy, France (1992).

[61] J. T. Linneman et al., The $\mathrm{D} \emptyset$ Software Trigger in Proceedings of the Conference on Computing in High Energy Physics, Annecy, France (1992).

[62] J. Butler, "Main Ring Deadtime," DØ Internal Note 1682 (1993), unpublished.

[63] M. Goosens et al., The ZEBRA System, CERN Library Program Entry Q100/Q101 (1995).

[64] S. Snyder, "The DØ Detector," DØ Internal Note 2500 (1995), unpublished.

[65] G. Manning, "Dø Software Documentation." (URL: http://d0wop.fnal.gov/software/offline_document.ps)

[66] N. Graf, DØ Internal Note (in preparation).

[67] T. Yasuda, "A Study of CDC z Measurement Using Collider Muon Tracks," DØ Internal Note 2782 (1995), unpublished.

[68] S. Glenn, A Search for Self Interactions of Neutral Electroweak Gauge Bosons. Ph.D. thesis, University of California at Davis, Davis, California, 1996 (unpublished).

[69] S. Youssef, "A Family of Cluster Algorithms," DØ Internal Note 375 (1986), unpublished. 
[70] S. Youssef, "Clustering with Local Equivalence Relations," DØ Internal Note 541 (1987), unpublished.

[71] N. Graf, "Simulation of the Level 1 CC Edge Effect," DØ Internal Note 1339 (1992), unpublished.

[72] N. Graf, "Improvements to the Calorimeter Electron Position Algorithm," D Internal Note 1352 (1992), unpublished.

[73] A. Goldschmidt and S. Glenn, "Corrections to Biases in Cluster-Track Matching," DØ Internal Note 2419 (1995), unpublished.

[74] R. Raja et al., "Reconstruction and Analysis: Trcaking Packages, Calorimeter Packages," DØ Internal Note 1006 (1990), unpublished.

[75] R. Raja, "H-matrix Analysis for top $\rightarrow$ lepton + jets," DØ Internal Note 1192 (1992), unpublished.

[76] M. Narain, Electron Identification in the D $\emptyset$ Detector, in Proceedings of the American Physical Society Division of Particles and Fields Conference, Fermilab, Batavia, Illinois (1992).

[77] F. Carminati et al., GEANT Users Guide, CERN Library Program Entry W5013 (1993).

[78] E. Flattum, A Measurement of the $W$ Boson Mass in $\bar{p}$ collisions at $\sqrt{\mathrm{s}}=1.8$ TeV. Ph.D. thesis, Michigan State University, East Lansing, Michigan, 1996 (unpublished).

[79] U. Heintz, "Standard EM Energy Scale for Reco V10," DØ Internal Note 1758 (1993), unpublished.

[80] The bad runs are eliminated using the RUN_SELECT package within the DØUSER framework (both are part of the standard DØ software library). The package looks for the bad runs in

$$
\text { DO\$PHYSICS_UTIL\$ROOT : [GENERAL] BAD_RUN . RCP }
$$

with the $\$ O F E$ hexadecimal mask, which selects all problem runs except those restricted to the muon system only. The RCP file is dated 13-SEP-1996.

[81] I. Adam, Measurement of the W Mass Using the Electron Transverse Energy Spectrum. Ph.D. thesis, Columbia University, New York, New York, 1997 (unpublished).

[82] G. Marchesini et al., Computer Physics Communications, 67, 465 (1992).

[83] P. B. Arnold and M. H. Reno, Nucl. Phys. B319, 37 (1989).

Erratum, Nucl. Phys. B330, 284 (1990).

[84] G. A. Ladinsky and C.-P. Yuan, Phys. Rev. D50, 4239 (1994).

[85] J. Collins, D. Sopper, and G. Sterman, Nucl. Phys. B250, 199 (1985). 
[86] F. A. Berends and R. Kleiss, Zeit. Phys. C27, 365 (1985).

[87] F. Paige and S. Protopopescu, ISAJET 5.20: A Monte Carlo Event Generator for $p p$ and $\bar{p} p$ Reactions. BNL-38034 (1996).

[88] I. Adam et al., "Observation of the $J / \psi \rightarrow e e$ decays at D $\varnothing$ and Calibration of the Central Calorimeter Electromagnetic Scale," DØ Internal Note 2298 (1994), unpublished.

[89] U. Heintz, "A Measurement of the Calorimeter Response using $\pi^{o}$ Decays," DØ Internal Note 2268 (1994), unpublished.

[90] W. G. D. Dhamaratna, "Reconstruction of the Calorimeter Response - Test Beam Load 2," DØ Internal Note 1731 (1993), unpublished.

[91] M. Bhattacharjee et al., "Jet Energy Resolutions," DØ Internal Note 2887 (1996), unpublished.

[92] A. D. Martin, R. G. Roberts, and W. J. Striling, Phys. Lett. 354B, 155 (1995).

[93] I. Adam, E. Flattum, U. Heintz and A. Kotwal, "W Boson Mass Measurement using Run 1b Data," DØ Internal Note 2982 (1997), unpublished.

[94] A. D. Martin, R. G. Roberts, and W. J. Striling, Phys. Lett. 356B, 89 (1995).

[95] M. Narain and U. Heintz, "A Study of Electron ID Efficiencies Using $Z \rightarrow e e$ Decays," DØ Internal Note 1814 (1993), unpublished.

[96] P. Grudberg, "Cross Section Measurements of $W$ and $W$ Bosons to Eletrons at DØ: Event Selection and Efficiency Studies," DØ Internal Note 2316 (1994), unpublished.

[97] W. Carvalho and T. Taylor, "Multiple Interaction Tool Study for Run 1," DØ Internal Note 2798 (1995), unpublished.

[98] J. Womersley, The D $\emptyset$ Monte Carlo, in Proceedings of the XXVI International Conference on High Energy Physis, Dallas, Texas (1992).

[99] T. Sjostrand, PYTHIA 5.7 and JETSET 7.4: Physics and Manual, CERNTH-7112-93 (1994).

[100] D. Casey, private communication.

[101] A. G. Frodesen and O. Skjeggestad, Probability and Statistics in Particle Physics. Universitetsforlaget (1979).

[102] J. Bunn and M. Goosens, HBOOK: Statistical Analysis and Histogramming, CERN Library Program Entry Y250 (1994).

[103] J. Bantly et al., "DØ Luminosity Monitor Constant for the 1994-96 Tevatron Run," FNAL Technical Memo Fermilab-TM-1995, (1997). 
[104] N. Amos et al., Phys. Lett. B243, 158 (1990).

[105] F. Abe et al., Phys. Rev. D50, 5518, 5535, 5550 (1994).

[106] P. Grudberg, Measurement of the $W$ and $Z$ Boson Production ross Sections in $p \bar{p}$ Cillisions at $\sqrt{\mathrm{s}}=1.8 \mathrm{TeV}$ with the DØ Detector. Ph.D. thesis, University of California at Berkeley, Berkeley, California, 1997 (unpublished).

[107] K. Goulianos, "Pomeron Flux Renormalization in Soft and Hard Diffraction." Rockefeller Preprint RU 95/E-06 (1995), submitted to Phys. Rev. Lett.

[108] F. Abe et al., Phys. Rev. D50, 5550 (1994).

[109] P. Aurenche et al., Phys. Rev. D45, 92 (1992).

[110] F. Bopp et al., Zeit. Phys. C51, 99 (1991).

[111] N. Amos et al., "Luminosity Calculations for DØ," DØ Internal Note 2031 (1994), unpublished.

[112] The author was responsible for providing these samples. Details on the actual process can be found in the WZ folder of DØNEws. (Messages \#389 and \#521 On FNALDO).

[113] M. Swartz, SLAC-PUB-5258 (1990).

[114] D. Amidei and R. Brock, "Future Electroweak Physics at the Fermilab Tevatron," FERMILAB-Pub-96/082 (1996). 